This document was prepared in conjunction with work accomplished under Contract No. DE-AC09-96SR18500 with the U. S. Department of Energy.

\title{
DISCLAIMER
}

This report was prepared as an account of work sponsored by an agency of the United States Government. Neither the United States Government nor any agency thereof, nor any of their employees, makes any warranty, express or implied, or assumes any legal liability or responsibility for the accuracy, completeness, or usefulness of any information, apparatus, product or process disclosed, or represents that its use would not infringe privately owned rights. Reference herein to any specific commercial product, process or service by trade name, trademark, manufacturer, or otherwise does not necessarily constitute or imply its endorsement, recommendation, or favoring by the United States Government or any agency thereof. The views and opinions of authors expressed herein do not necessarily state or reflect those of the United States Government or any agency thereof.

This report has been reproduced directly from the best available copy.

Available for sale to the public, in paper, from: U.S. Department of Commerce, National Technical Information Service, 5285 Port Royal Road, Springfield, VA 22161, phone: (800) 553-6847, fax: (703) 605-6900

email: orders@ntis.fedworld.gov

online ordering: http://www.ntis.gov/help/index.asp

Available electronically at http://www.osti.gov/bridge

Available for a processing fee to U.S. Department of Energy and its contractors, in paper, from: U.S. Department of Energy, Office of Scientific and Technical Information, P.O. Box 62, Oak Ridge, TN 37831-0062,

phone: (865)576-8401,

fax: (865)576-5728

email: $\underline{\text { reports@ adonis.osti.gov }}$ 
WSRC-TR-2002-00550, Rev. 0

SRT-RPP-2002-00274

KEYWORDS:

River Protection Project Laboratory

Evaporation

Offgas Emissions

RETENTION TIME:

Permanent

\section{Waste Feed Evaporator Off-Gas Emissions Modeling}

Authors:

Issue Date:

Test Specification No.

Test Plan No.

Test Scoping Statement:
William E. Daniel, 999-W, SRTC, SRS, 803-819-8463

December 4, 2002

24590-PTF-TSP-RT-01-006, Rev. 0

WSRC-TR-2002-00262, Rev. 0

S-89

Westinghouse Savannah River Company

Savannah River Site

Aiken, SC 29808

Prepared for the U.S. Department of Energy Under Contract Number DE-AC09-96SR18500

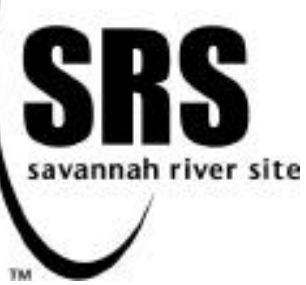


This page intentionally left blank 
This report describes the results of work and testing specified by 24590-WTPTSP-RT-01-006, Rev. 0 and WSRC-TR-2002-00262, Rev. 0. . The performed work followed established quality assurance requirements and was conducted as authorized. The descriptions provided in this test report are an accurate account of both the conduct of the work and the data collected. Results required by the test plan are reported. Also reported are any unusual or anomalous occurrences that are different from starting hypotheses. The test results and this report have been reviewed and verified. 


\section{TABLE OF CONTENTS}

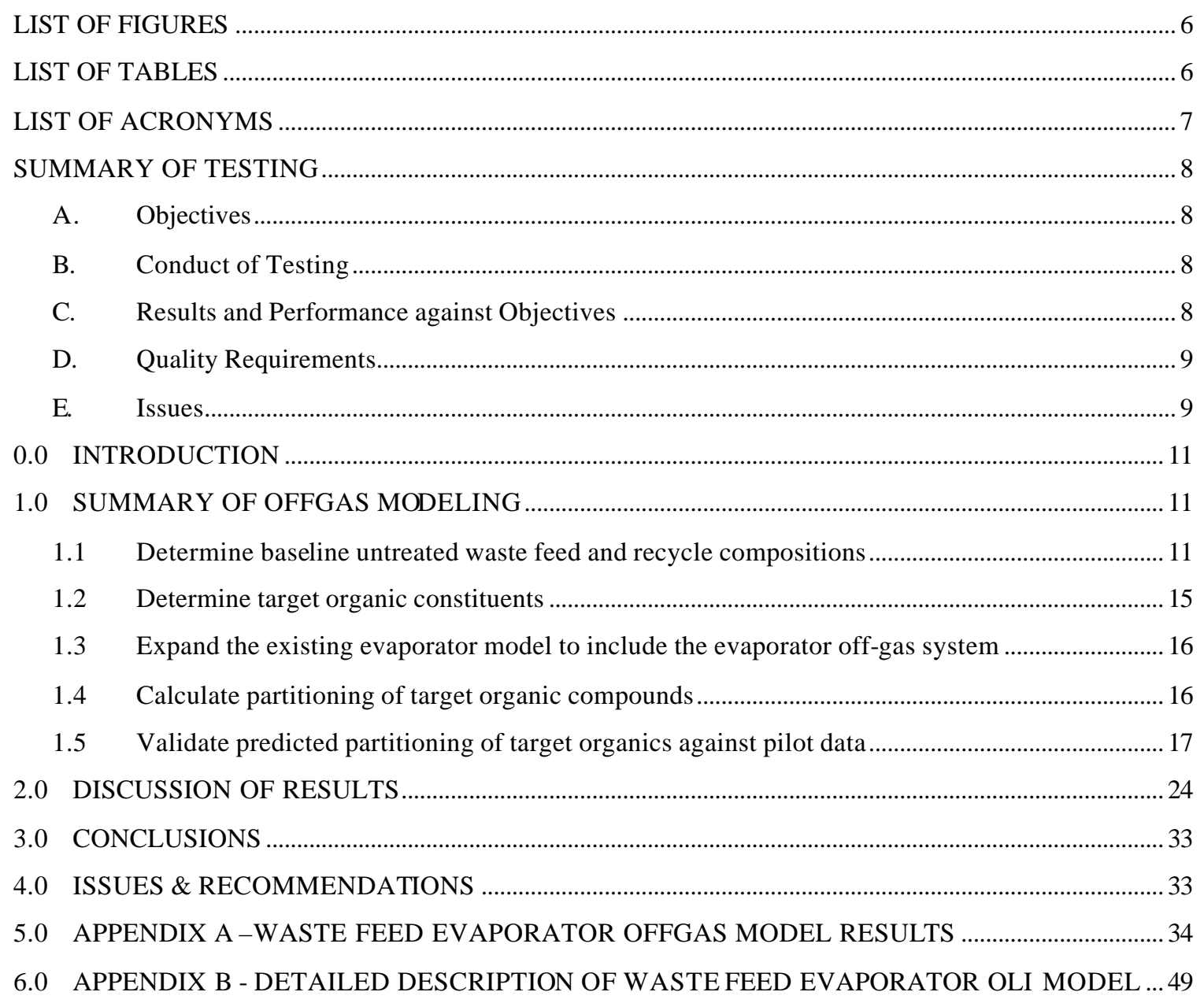




\section{LIST OF FIGURES}

Figure 1. Results: Mass Flows, Temperatures, Pressures for Offgas Regulatory Run for 1.22 density for UF1 Filtrate Stream of Waste Feed Evaporator.

Figure 2. Results: Mass Flows, Temperatures, Pressures for Offgas Regulatory Run for 1.35 density for UF1 Filtrate Stream of Waste Feed Evaporator.

Figure A-1. Organic Partitioning for Offgas Regulatory Run UF1 Filtrate 1.22 density.................................................30

Figure A-2. Organic Partitioning for Offgas Regulatory Run UF1 Filtrate 1.35 density.

Figure B-1. Waste Feed Evaporator Model Overview: Evaporator, Ultrafiltration Phase $1 \& 2$, and First \& Second Caustic Washes, and Total Recycle

Figure B-2. Waste Feed Evaporator OLI Model Diagram - Evaporator, Ultrafiltration Phase 1, and First \& Second Caustic Wash Splits.

Figure B-3. Waste Feed Evaporator OLI Model Diagram - First Caustic Wash (Broken into 5 phases FCW1-FCW5 to simulate multiple volume transfers)

Figure B-4. Waste Feed Evaporator OLI Model Diagram- Leach, Ultrafiltration Phase 2, Caustic and Acid Rinse, Total Recycle, and Second Caustic Wash (broken into 5 phases SCW1-SCW5 to simulate multiple volume transfers).

\section{LIST OF TABLES}

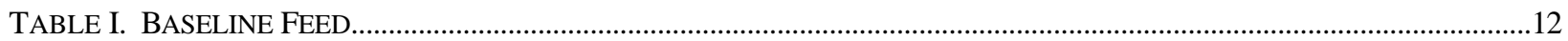

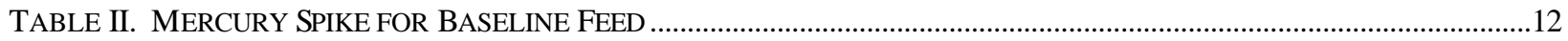

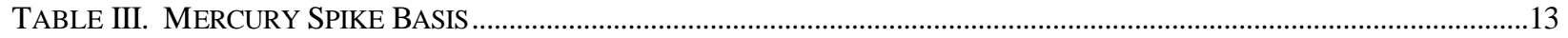

TABLE IV. OLI MERCURY EQUILIBRIUM FOR FEED................................................................................................13

TABLE V. EQUILIBRIUM INPUT AND REDUCED FEEDS WITH MERCURY AT $25^{\circ} \mathrm{C}, 1$ ATM ..................................................14

TABLE VI. TARGET ORGANIC COMPOUNDS SPIKED INTO ENVELOPE B/D FEED ………………………………………….....15

TABLE VII. ORGANIC FEED FLOWS FOR OFFGAS REGULATORY MODELING RUN UF1 FILTRATE 1.22 DENSITY.............19

TABLE VIII. ORGANIC PARTITIONING IN TERMS OF W ASTE FEED AND RECYCLE STREAMS FOR OFFGAS REGULATORY MODELING RUN UF1 FILTRATE 1.22 DENSITY...................................................................................................20

TABLE IX. ORGANIC FEED FLOWS FOR OFFGAS REGULATORY MODELING RUN UF1 FILTRATE 1.35 DENSITY .............22

TABLE X. ORGANIC PARTITIONING IN TERMS OF W ASTE FEED AND RECYCLE STREAMS FOR OFFGAS REGULATORY

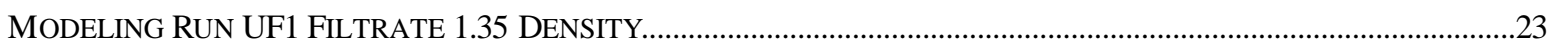

TABLE XI. COMPARISON OF HIROSHI AND FEP MODELING RUNS..........................................................................................2

TABLE XII. ORGANIC AND MERCURY PARTITIONING OF MODELING UlTRAFILTRATE 1.22 DENSITY CASE ...................27

TABLE XIII. ORGANIC AND MERCURY PARTITIONING OF MODELING ULTRAFILTRATE 1.35 DENSITY CASE ................28

TABLE XIV. ORGANIC SPLITS COMPARED WITH EARLIER MODELING WORK …………………………............................29

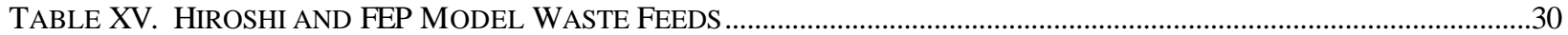

TABLE XVI. HIROSHI AND FEP MODEL W ASTE FEED COMPOSITIONS..................................................................................30

TABLE A-1. WASTE FEED EVAPORATOR OFFGAS MODEL OUTPUT FOR UF1 FILTRATE DENSITY OF 1.22 G/ML.........31

TABLE A-2. WASTE FEED EVAPORATOR OFFGAS MODEL OUTPUT FOR UF1 FILT RATE DENSITY OF 1.35 G/ML .........38

TABLE B-1. WASTE FEED EVAPORATOR 5 STAGE MODEL RESULTS FOR UF 1.22 DENSITY CASE ..............................49

TABLE B-2. WASTE FEED EVAPORATOR 5 STAGE MODEL RESULTS FOR UF 1.35 DENSITY CASE ................................69 


\section{LIST OF ACRONYMS}

$\begin{array}{ll}\text { AA } & \text { Atomic Absorption } \\ \text { FEP } & \text { Feed Evaporator Process } \\ \text { HLW } & \text { High Activity Waste } \\ \text { IC } & \text { Ion Chromatography } \\ \text { ICPES } & \text { Inductively Coupled Plasma Emissions Spectroscopy } \\ \text { LAW } & \text { Low Activity Waste } \\ \text { RPP } & \text { River Protection Project } \\ \text { SRTC } & \text { Savannah River Technology Center } \\ \text { SBS } & \text { Submerged Bed Scrubber } \\ \text { TOC } & \text { Total Organic Carbon } \\ \text { UF } & \text { Ultrafiltration } \\ \text { WTP } & \text { Waste Treatment Plant } \\ \text { XRD } & \text { X-ray Diffraction }\end{array}$




\section{Summary of Testing}

\section{A. Objectives}

The goals of this task were:

A. Expand the waste feed evaporator model by including all the major components of the evaporator off-gas treatment system

B. Develop two test cases by defining the baseline untreated waste feed composition, target organic components to be tracked, and the waste-to-recycle blend ratio

C. Develop air emissions projections for the two test cases using the expanded waste feed evaporator model

D. Validate organic partitioning predictions and air emission projections against experimental data obtained during pilot evaporator tests

The first three objectives were accomplished and objective D will be performed once the pilot test data is available.

\section{B. Conduct of Testing}

The Environmental Simulation Program (ESP) software licensed by OLI Systems, Inc. was used to model the waste feed evaporator and the offgas treatment system. OLI ESP is a steady state simulation package that performs vapor/liquid/solid equilibrium for electrochemical streams and processes. For the waste feed evaporator and offgas modeling the following private OLI databooks were used: Carbonat, Zeolite, Silica, and HNO3DB. The details for the waste feed evaporator model are discussed in another task plan ${ }^{1}$. The baseline waste feed and SBS recycle stream data was provided by Alex Choi from analytical data for the representative Envelope B/D samples ${ }^{2,3}$, whose TOC levels are lower than those of Envelope C. To bound the air emission projections, the baseline Envelope B/D feeds were spiked in this task with additional organics to increase the total organic carbon (TOC) level to that of Envelope C or approximately 1 PPM. The waste feed rate was set to produce $30 \mathrm{Mton} /$ day of $17 \mathrm{wt} \% \mathrm{Na}_{2} \mathrm{O}$ glass.

\section{Results and Performance against Objectives}

In a prior study ${ }^{4,5}$ fourteen target organic species representing the volatile, semi-volatile, and nonvolatile organics of the waste feeds were spiked into the treated evaporator feed. The partitioning of these organics in the evaporator pot and in the primary condenser was measured in lab-scale equipment. The lab measured values agreed well with the OLI ESP model predictions for most organics except pentachlorophenol, pyrene, and Aldrin. This prior study showed that all volatiles and light semi-volatiles would exit the evaporator system through the off-gas, while the heavier semi-volatiles remain predominantly in the evaporator concentrate. The offgas regulatory runs for this task were compared with this prior study to help validate the model. The current offgas runs showed that more of the volatiles and semi -volatiles in the feed go out the condensate stream of the evaporator than the earlier runs due to the large amount of HLW Offgas Condensate (SBS Recycle) being sent to the waste feed evaporator. The extra water

\footnotetext{
${ }^{1}$ Laurinat, J. E., and A. S. Choi, "Task Technical and Quality Assurance Plan for Waste Feed Evaporation and Physical Properties Modeling," WSRC-TR-2002-00083, SRT-RPP-2002-00036, Rev. 0, Westinghouse Savannah River Co., Aiken, SC, April, 2002.

${ }^{2}$ Schreiber, Ruth D., "Tank Characterization Report for Double-Shell Tank 241-AZ-102”, WHC-SD-WM-ER-411 Rev. 0-A, Westinghouse Hanford Company, Richland, WA, December 1995.

${ }^{3}$ Tank Farm Contractor Operation and Utilization Plan (TFCOUP), HNF-SD-WM-SP-012, Rev. 3, Numatec Hanford Corporation and CH2MHILL Hanford Group, Inc., Richland, WA, September 1991.

${ }^{4}$ Saito, H. H., T. B. Calloway, D. M. Ferrara, A. S. Choi, T. L White, L. V. Gibson, and M. A. Burdette, "Simulant Bench-Scale LAW Evaporation with Organic Regulatory Analysis," SRT-RPP-2000-00047, Westinghouse Savannah River Co., Aiken, SC, 2001.

5 Choi, A. S., "Preliminary Modeling Results of Pretreated LAW Evaporator," BNF-003-98-0080, Rev. 0, Westinghouse Savannah River Co., Aiken, SC, June 1, 1999.
} 
and species in the HLW Offgas Condensate stream caused the vapor/liquid equilibrium according to OLI to shift in the evaporator so more of the volatiles and semi-volatiles go out the evaporator overhead and end up being condensed out in the primary condenser. Several runs were made with the prior OLI model and the new waste feed evaporator offgas model to confirm this behavior. From these comparisons, adjusting the evaporator vapor target does have a significant impact on the organic partitioning. The amount of water entering the evaporator from the recycle streams also has a significant impact.

This work does not reflect the true conditions of mercury in the waste feed evaporator. For this reason it is strongly suggested that the mercury calculations not be used in the evaporator engineering calculations for design. SRTC will be conducting experiments to attempt to provide a mercury mass balance across the waste feed evaporator. ${ }^{6}$ This new work will be better suited for mercury analysis and engineering design calculations.

The mercury partitioning in the offgas system for the conditions assumed is fairly straightforward based on the OLI modeling. Any elemental mercury in the feed goes out the evaporator overhead. For this particular model setup soluble mercury was represented as mercuric chloride. About $5 \%$ of the mercuric chloride goes out the overhead while the remaining portion goes out the evaporator bottoms. No other forms of mercury were predicted to go out the evaporator overhead other than elemental mercury and mercuric chloride. All the insoluble mercury goes out the evaporator bottoms. In these OLI models the insoluble mercury was represented as mercuric oxide. How much mercury that's predicted to go out the evaporator overhead depends on the assumed amount of elemental mercury in the feed and the assumed reduction of insoluble mercury in the feed to elemental mercury. For the offgas regulatory runs a 50\% reduction of mercuric oxide to elemental mercury was assumed to show the effect of mercury redox. The actual RPP feed has not been shown to have any potential to reduce mercury. This assumption of mercury redox was made to show the effect should it occur. If there is no elemental mercury in the waste feed and no mercury redox is assumed for the waste feed then OLI predicts essentially no mercury will go out the overhead stream. Any insoluble mercury in the waste feed that is not reduced will go out the evaporator bottoms based on OLI predictions.

\section{Quality Requirements}

The quality requirements for this work were documented in the Task Technical and Quality Assurance Plan for Waste Feed Evaporator Off-Gas Emissions Modeling (WSRC-TR-2002-00262). The WSRC Quality Assurance Program was followed, which has been approved by WTP, and the WSRC Quality Assurance Management Plan (WSRC-RP-92-225). This program applied the appropriate quality assurance requirements for this task from NQA1-1989, and NQA-2a-1990, Part 2.7, as indicated by the QA plan (WSRC-TR-2002-00262). Modeling results were compared to prior modeling and experimental results to confirm model predictions.

\section{E. Issues}

In gathering data for the two offgas runs, several runs were done to investigate mercury redox. Based on these runs it appears that the built-in redox for mercury must be modified to more closely match observed mercury redox behavior. Some redox behavior can be inserted into the OLI chemistry model by using kinetic equations. However, the reduction and oxidation are then forced to fit a desired result rather than following standard redox chemistry. The mercury redox behavior in OLI should be studied further so it can be refined to more closely match experimental data. Further, the behavior of soluble and insoluble forms of mercury in OLI needs to be investigated to address concerns over the partitioning of mercury between the evaporator overhead and bottoms.

\footnotetext{
${ }^{6}$ Stone, M. A. "Task Technical and Quality Assurance Plan for Waste Feed: Simulant Evaporation and Physical Properties Determination.” WSRC-TR-2002-00039, SRT-RPP-2002-00013, Westinghouse Savannah River Company, Aiken, SC, 2002.
} 
In collecting data for the two offgas runs, several runs were made with different amounts of water coming into the offgas train from the feed streams as well as the steam ejectors and recycle streams. The organic partitioning is significantly impacted by the amount of water being fed into the system due to the volatility of the organics and their solubility in water. The amount of water can be controlled by the amount of excess steam going to the steam ejectors as well as the amount of HLW offgas condensate (SBS Recycle) and Ultrafiltration caustic wash that are recycled back to the evaporator. More studies are needed to quantify the impacts of these variables. 


\section{WSRC-TR-2002-00550, Rev. 0 \\ SRT-RPP-2002-00274}

\subsection{INTRODUCTION}

The objectives of this task given in the test specification were to expand the waste feed evaporator OLI model by including all the major components of the evaporator off-gas treatment system. Also as part of this task, two test cases were developed that defined the baseline untreated waste feed composition, target organic components to be tracked, and the waste-to-recycle blend ratio. The goal of this task was to develop air emissions projections for two test cases using the expanded waste feed evaporator OLI model. The organic partitioning predictions and air emission projections are ultimately to be validated against experimental data obtained during pilot evaporator tests.

The Environmental Simulation Program (ESP) software, licensed by OLI Systems, Inc., was used to build the current as well as earlier models for the $\mathrm{FEP}^{7}$. The steady state simulation package performs vapor/liquid/solid equilibrium for electrochemical streams and processes. All vapor/liquid/solid equilibrium is determined by the OLI simulation engine. OLI uses public and private databooks of component data to predict various components' properties. The private databooks used in this modeling task were Carbonat, Zeolite, Silica, and HNO3DB. These databooks were developed in cooperation with OLI Systems.

This task partially satisfied the test conditions and requirements described in Section 3.4 of the Research and Technology Plan, (PL-W375-TE00007, Rev. 0) prepared by Bechtel National, Inc. (BNI). Specifically, BNI will use the information obtained during this task to support environmental regulatory permitting efforts for the waste feed evaporator and further to provide the operating data and correlations for the RPP-WTP flowsheet model. The overall scope of this task was described in the WTP R\&T Test Scoping Statement S89.

This report completes the activities of the Task Technical and Quality Assurance Plan for Waste Feed Evaporator Off-Gas Emissions Modeling (WSRC-TR-2002-00262). The WSRC Quality Assurance Program was followed, which has been approved by WTP, and the WSRC Quality Assurance Management Plan (WSRC-RP-92-225). This program will apply the appropriate quality assurance requirements for this task from NQA-1-1989, and NQA-2a-1990, Part 2.7, as indicated by the QA plan (WSRC-TR-200200262).

\subsection{SUMMARY OF OFFGAS MODELING}

A task and quality assurance plan was developed and carried out to produce modeling data in support of the regulatory offgas permitting. The tasks involved to produce this offgas data consisted of the following:

\subsection{Determine baseline untreated waste feed and recycle compositions}

The RPP-WTP pretreatment has undergone several major flowsheet changes recently, which include bypassing of the waste feed evaporator altogether when the sodium content of untreated waste feeds is greater than 5 molar. The baseline waste feed and SBS recycle stream data was provided by Alex Choi from analytical data for the representative Envelope B/D samples ${ }^{2,3}$, whose TOC levels are lower than those of Envelope C. The waste feed rate was set to produce $30 \mathrm{Mton} /$ day of $17 \mathrm{wt} \% \mathrm{Na}_{2} \mathrm{O}$ glass. The TOC level of the baseline feed was increased to the Envelope $\mathrm{C}$ levels to bound the air emission projections. The baseline feed was also spiked with both soluble and insoluble mercury. The un-spiked baseline feed compositions are shown in Table I. The organic spike amounts are shown in the next section. The mercury spike for the waste feed is shown in Table II. The insoluble mercury was represented by mercuric oxide $(\mathrm{HgO})$ and the soluble mercury was represented by mercuric chloride $\left(\mathrm{HgCl}_{2}\right)$. These forms were chosen based on their solubility behavior in the OLI modeling software. To determine the amount of mercury to spike in the waste feed, all insoluble and soluble mercury levels in the tank farm were reviewed based on TFCOUP data ${ }^{3}$.

\footnotetext{
${ }^{7}$ ESP Software, http://www.olisystems.com/, OLI Systems, Inc., Morris Plains, NJ (2002).
} 
Table III shows the results of the tank survey. Tank AZ-102 had the highest soluble Hg content with respect to the total soluble solids in each tank. Tank AY-102 had the highest insoluble Hg content with respect to the total insoluble solids in each tank. These tank levels were used in conjunction with contract limits on the amount of mercury to be processed to derive the mercury spikes for the waste feed. The contract limit for insoluble mercury is $0.1 \mathrm{~g} \mathrm{Hg}$ per $100 \mathrm{~g}$ nonvolatile oxide and the assumed total gram equivalents of non-volatile oxide per liter of HLW sludge is 31 . Combining these two values gives a $0.03 \mathrm{~g}$ insoluble $\mathrm{Hg}$ per liter of waste feed. The volume of the waste feed was 3560 liter per hour. These two values gave the amount of insoluble mercury in the feed. The total insoluble solids in the feed were then calculated based on the amount of insoluble mercury in the feed and the Tank AY-102 insoluble mercury ratio. The contract limit for soluble mercury was $1.4 \mathrm{e}-5$ gmole soluble $\mathrm{Hg}$ per gmole $\mathrm{Na}$ from Appendix A of the TFCOUP document ${ }^{3}$. Using this contract limit gives a soluble $\mathrm{Hg}$ to total soluble solids ratio of $3.08 \mathrm{e}-5$ in the waste feed compared to the Tank AZ-102 value of 3.83e-7. To be conservative the higher contract limit value was used to set the amount of soluble mercury in the feed. If this assumption is not valid then a different amount of soluble mercury should be assumed. The actual mercury feed amounts shown in Table II were derived from these mercury tank values and contract limits.

Table I. Baseline Feed

\begin{tabular}{|c|c|c|}
\hline Real Name & OLI Name & GMOLES \\
\hline Water & H2O & $1.880 \mathrm{E}+05$ \\
\hline Sodium Nitrite & NANO2 & $2.906 \mathrm{E}+03$ \\
\hline Sodium Nitrate & NANO3 & $6.503 \mathrm{E}+02$ \\
\hline Sodium Hydroxide & NAOH & $3.921 \mathrm{E}+02$ \\
\hline Sodium Sulfate & NA2SO4 & $5.872 \mathrm{E}+02$ \\
\hline Sodium Oxalate & NAALO2 & $1.122 \mathrm{E}+02$ \\
\hline Sodium Aluminate & NAF & $1.740 \mathrm{E}+01$ \\
\hline Sodium Fluoride & NACL & $1.792 \mathrm{E}+01$ \\
\hline Sodium Chloride & NA2CO3 & $1.783 \mathrm{E}+03$ \\
\hline Sodium Carbonate & NA3PO4 & $9.005 \mathrm{E}+00$ \\
\hline Sodium Phosphate & NA2CRO4 & $5.155 \mathrm{E}+01$ \\
\hline Sodium Chromate (VI) & KNO3 & $2.812 \mathrm{E}+02$ \\
\hline Potassium Nitrate & NACOOH & $8.605 \mathrm{E}+00$ \\
\hline Sodium Formate & H2IDA & $8.507 \mathrm{E}+00$ \\
\hline 2,2-Iminobisacetic acid & NAACET & $3.264 \mathrm{E}+00$ \\
\hline Sodium Acetate & NAH2CTRT & $9.214 \mathrm{E}+00$ \\
\hline Sodium Dihydrogen Citrate & NAGLYCOLAT & $4.648 \mathrm{E}+01$ \\
\hline Sodium Glycolate & NA2H2EDTA & $5.506 \mathrm{E}+00$ \\
\hline Disodium Dihydrogen EDTA & CANO32 & $9.404 \mathrm{E}+00$ \\
\hline Calcium Nitrate & CSNO3 & $1.027 \mathrm{E}+00$ \\
\hline Cesium Nitrate
\end{tabular}

Table II. Mercury Spike for Baseline Feed ${ }^{8}$

\begin{tabular}{|c|c|c|}
\hline Real Name & OLI Name & GMOLES \\
\hline Mecuric Chloride (sol) & HGCL2 & $9.600 \mathrm{E}-02$ \\
\hline Mercuric Oxide (insol) & HGO & $5.320 \mathrm{E}-01$ \\
\hline
\end{tabular}

\footnotetext{
${ }^{8}$ This work does not reflect the true conditions of mercury in the waste feed evaporator. For this reason it is strongly suggested that the mercury calculations not be used in the evaporator engineering calculations for design.
} 
WSRC-TR-2002-00550, Rev. 0

SRT-RPP-2002-00274

Table III. Mercury Spike Basis ${ }^{8}$

\begin{tabular}{|c|c|c|c|}
\hline \multicolumn{2}{|c|}{ Highest Soluble Hg Concentration } & Highest Insoluble Hg Concentration \\
\hline \multicolumn{2}{|c|}{ Tank AZ-102 } & \multicolumn{2}{c|}{ Tank A Y-102 } \\
\hline $\begin{array}{c}\text { Tank Soluble Hg/ } \\
\text { Total Soluble Solids }\end{array}$ & $3.83 \mathrm{e}-7$ & $\begin{array}{c}\text { Tank Insoluble Hg/ Total } \\
\text { Insoluble Solids }\end{array}$ & $6.28 \mathrm{e}-4$ \\
\hline $\begin{array}{c}\text { Model Soluble Hg/ } \\
\text { Total Soluble Solids }\end{array}$ & $3.08 \mathrm{e}-5^{*}$ & $\begin{array}{c}\text { Model Insoluble Hg/ } \\
\text { Total Insoluble Solids }\end{array}$ & $6.29 \mathrm{e}-4$ \\
\hline
\end{tabular}

Higher than tank value due to $1.4 \mathrm{e}-5 \mathrm{gmol}$ sol $\mathrm{Hg} / \mathrm{gmol} \mathrm{Na}$ contract limit

Due to the basic nature of the waste feed, the insoluble mercury and soluble mercury feed input values shown in Table II change when OLI calculates equilibrium The primary OLI equilibrium equations for mercury for this model were:

$$
\begin{aligned}
& \mathrm{HgO}+\mathrm{H} 2 \mathrm{O} \leftrightarrow \mathrm{Hg}^{2+}+2 \mathrm{OH}^{-} \\
& \mathrm{HgCl}_{2} \leftrightarrow \mathrm{Hg}^{2+}+2 \mathrm{Cl}^{-} \\
& \mathrm{Hg}(\mathrm{OH})_{2} \leftrightarrow \mathrm{Hg}^{2+}+2 \mathrm{OH} \\
& \mathrm{Hg}(\mathrm{OH})_{3} \leftrightarrow \leftrightarrow \mathrm{Hg}^{2+}+3 \mathrm{OH}
\end{aligned}
$$

The OLI mercury equilibriumfor the waste feed is shown in Table IV. Forty percent of the mercuric oxide stays in its input form while the remaining $60 \%$ converts to mercuric hydroxide $\left(\mathrm{Hg}(\mathrm{OH})_{2}\right)$ and mercury trihydroxide ion $\left(\mathrm{Hg}(\mathrm{OH})_{3}{ }^{-1}\right)$. Note that all mercury species have a valence of 2. At equilibrium the mercuric oxide form is insoluble and the hydroxide forms are soluble. In this sense, a portion of the feed mercuric oxide is soluble and another portion is insoluble. The mercuric chloride in the feed input is converted to mercuric hydroxide $\left(\mathrm{Hg}(\mathrm{OH})_{2}\right)$ and mercury trihydroxide ion $\left(\mathrm{Hg}(\mathrm{OH})_{3}^{-1}\right)$ on equilibrium Therefore OLI feed input values are not necessarily fixed as OLI will perform an equilibrium calculation for all species in the feed stream before any calculations start. In this sense the soluble mercury in the waste feed is represented by its proper mercuric forms $\left(\mathrm{Hg}(\mathrm{OH})_{2}\right.$ and $\left.\mathrm{Hg}(\mathrm{OH})_{3}{ }^{-1}\right)$.

Table IV. OLI Mercury Equilibrium for Feed ${ }^{8}$

\begin{tabular}{|c|c|c|}
\hline HG Compound & $\begin{array}{c}\text { Waste Feed Input } \\
\text { [mole/hr] }\end{array}$ & $\begin{array}{c}\text { Waste Feed } \\
\text { OLI Equil. [mole/hr] }\end{array}$ \\
\hline $\mathrm{HgO}$ (insoluble) & 0.532 & 0.213 \\
\hline $\mathrm{HgCl}{ }_{2}$ (soluble) & 0.096 & 0 \\
\hline $\mathrm{Hg}(\mathrm{OH})_{2}$ (soluble $)$ & 0 & 0.399 \\
\hline $\mathrm{Hg}(\mathrm{OH})_{3}{ }^{-1}$ (soluble) & 0 & 0.016 \\
\hline
\end{tabular}

To represent mercury redox in the model a mercury redox reaction was added to the OLI model chemistry. The redox reaction used was:

$$
\mathrm{NO}_{2}^{-}+\mathrm{Hg}^{+2}+2 \mathrm{OH}=\mathrm{Hg}+\mathrm{NO}_{3}^{-}+\mathrm{H}_{2} \mathrm{O}
$$

This redox reaction was based on prior experience with mercury redox in nitric acid feeds of evaporators at DWPF. The redox reaction was expressed as the following rate expression:

$$
\text { rate }=0.67 * \mathrm{HgO}
$$

where rate is in gmole/hr and $\mathrm{HgO}$ is in gmole/hr. This specific rate expression was chosen after trying several forms of the rate expression. Theoretically the redox rate could be expressed as:

$$
\text { rate }=0.5 * \mathrm{Hg}^{+2} * \mathrm{H}_{2} \mathrm{O} / 55.508
$$

where $\mathrm{Hg}^{+2}$ is in gmole/kg water and $\mathrm{H}_{2} \mathrm{O}$ is in gmole/hr to give the rate units of gmole/hr. However, after several trial runs, the rate expression in terms of the input species $\mathrm{HgO}$ gave the most predictable results. The 0.67 rate constant was chosen to give an assumed $50 \%$ reduction of $\mathrm{HgO}$ to $\mathrm{Hg}$ in the feed. This redox 
rate was purely arbitrary and was chosen to examine OLI's response for a non-zero redox behavior. The redox rate can be changed to better represent the RPP feed conditions.

OLI will perform the mercury redox defined above followed by its standard equilibriumcalculations. This results in a reaction pathway like:

$$
\mathrm{HgO} \rightarrow \mathrm{Hg} \leftrightarrow \mathrm{Hg}(\mathrm{OH})_{2}+\mathrm{Hg}(\mathrm{OH})_{3}^{-}
$$

Following the reaction pathway shown above, the OLI model streams with mercury (waste feed and total recycle) have the compositional makeup shown in Table V. The mercuric oxide in the feed is reduced first to elemental mercury $(\mathrm{Hg})$ according to the user specified redox rate. Any remaining mercuric oxide then equilibrates to mercuric hydroxide and mercury trihydroxide ion. The elemental mercury formed from reduction also equilibrates with mercuric hydroxide and mercury trihydroxide ion. This equilibration is why the amount of elemental mercury is less than the rate expression predicts. Since the mercury redox rate was arbitrarily set at $50 \%$, it could be set to $0 \%$ which would mean no elemental mercury would appear in the waste or recycle streams. Indirectly this redox specification affects the mercury partitioning in the offgas system since all elemental mercury in the OLI feed will go out the evaporator overhead. Only about $5 \%$ of the mercuric chloride in the evaporator feed will go out the overhead with $95 \%$ going out the bottoms. No other forms of mercury are predicted in the evaporator overhead stream. All forms of mercuric oxide (mercuric hydroxide and mercury trihydroxide ion) in the OLI feed will go out the evaporator bottoms. If there is concern for mercury in the offgas system then the amount of elemental mercury in the feed as well as any potential reduction of insoluble mercury in the feed should be carefully monitored and controlled.

Table V. Equilibrium Input and Reduced Feeds with Mercury at $25^{\circ} \mathrm{C}, 1 \mathbf{a t m}^{8}$

\begin{tabular}{|c|c|c|c|}
\hline HG Compound & $\begin{array}{c}\text { Total Recycle } \\
\text { OLI Equil. } \\
\text { [mole/hr] }\end{array}$ & $\begin{array}{c}\text { Waste Feed } \\
\text { OLI Equil. } \\
\text { [mole/hr] }\end{array}$ & $\begin{array}{c}\text { Waste Feed + Total } \\
\text { Recycle Reduced } \\
\text { OLI Equil. } \\
\text { [mole/hr] }\end{array}$ \\
\hline $\mathrm{HgO}$ (insoluble) & 0 & 0.213 & 0 \\
\hline $\mathrm{HgCl}{ }_{2}$ (soluble) & 0 & 0 & 0 \\
\hline $\mathrm{Hg}(\mathrm{OH})_{2}$ (soluble) & 0.004 & 0.399 & 0.312 \\
\hline $\mathrm{Hg}(\mathrm{OH})_{3}{ }^{-1}$ (soluble) & 0 & 0.016 & 0.004 \\
\hline $\mathrm{Hg}($ vapor $)$ & 0 & 0 & 0.316 \\
\hline
\end{tabular}

Secondary-waste recycle streams als o make up part of the feed to the waste feed evaporator system. The major recycle streams are the HLW melter submerged bed scrubber (SBS) condensate (also referred to as the HLW Offgas Condensate stream), ultrafiltration (UF) solids wash permeate and acidic/caustic cleaning solutions, ion-exchange resin wash effluents, and evaporator condensate ${ }^{9}$. The feed evaporator process (FEP) flowsheet including the UF and recycle blending was modeled in another task and a description of the FEP flowsheet is given in the test plan for that task ${ }^{1}$. The recycle and waste feed blends and flow rates of the prior task were used in this task. The FEP OLI model of the prior task was expanded to include the primary off-gas system for this work. The selection of the recycle composition and blending ratio for the two offgas test cases came out of the prior FEP flowsheet task ${ }^{1}$.

The two regulatory offgas test cases set the density of the post-UF clarified feed to the cesium ionexchange operation to the design basis of $1.22 \mathrm{~g} / \mathrm{ml}$ and the out-of-design point of $1.35 \mathrm{~g} / \mathrm{ml} .{ }^{10}$ Each density

\footnotetext{
${ }^{9}$ Bergman, L. M., E. Berrios, E. Bixel, D. Dearlove, R. Gimpel, C. Knauss, A. Olander, A. Pajunen, J. Reynolds, and D. Harty, "WTP Material Balances and Process Flowsheet Bases, Requirements, and Results,” 24590-WTP-RPT-ENG-01-004, Rev. 0, Washington Group International, Richland, WA, January, 2002.

${ }^{10}$ Nowak, R., "System Description for Waste Feed Evaporation Process (FEP)," 24590-PTF-3YD-FEP00001, Rev. B, Bechtel National, Inc., Richland, WA, May 1, 2002.
} 


\section{WSRC-TR-2002-00550, Rev. 0 \\ SRT-RPP-2002-00274}

point was achieved by changing the concentration or boilup in the evaporator. This boil-up in turn affected the organic partitioning.

\subsection{Determine target organic constituents}

Fourteen target organic compounds were spiked into the AN-107 simulant during a recent treated feed evaporator regulatory study by Hiroshi Saito ${ }^{11}$. These organic compounds were selected to represent volatile, semi-volatile, and pesticide contaminants present in the Hanford wastes. The volatile. semivolatile, and pesticide groups were defined in the Hiroshi study. The distinction between the groupings is based primarily on boiling points of the various components. The fourteen organics in the Hiroshi study were included in this study along with six additional organics shown in Table VI (the additional organics are bolded and italicized). All twenty target organic compounds listed in Table VI were spiked into the baseline Envelope B/D waste feed at 1 PPM each. The gmole amounts equivalent to 1 PPM are shown in Table VI. The 1 PPM amounts are based on a total mass including soluble and insoluble solids in the waste feed. The total soluble solids were made up of the baseline feed components minus the water plus the soluble portion of mercury. The total insoluble solids was based on the ratio of insoluble mercury to total insoluble solids in tank AY-102 (the highest mercury value). Using the total soluble and insoluble solids plus water weight, the organic masses were targeted at 1e-6 mass fraction from which the molar amounts were calculated.

Table VI. Target Organic Compounds Spiked in to Envelope B/D Feed

\begin{tabular}{|c|c|c|c|c|c|c|}
\hline & Target Organic & OLI Name & Formula & $\begin{array}{l}\text { Boiling Pt. } \\
{ }^{\circ} \mathrm{C}\end{array}$ & M.W. & $\begin{array}{l}\text { Spike } \\
\text { gmoles }\end{array}$ \\
\hline \multirow[t]{8}{*}{ Volatile } & Benzene & BENZENE & C6H6 & 80.1 & 78.11 & 0.053707 \\
\hline & $\begin{array}{l}\text { 4-methyl-2-pentanone } \\
\text { (MIBK) }\end{array}$ & M4PNTON2 & C6H12O & 116.5 & 100.16 & 0.041885 \\
\hline & Toluene & TOLUENE & $\mathrm{C} 7 \mathrm{H} 8$ & 110.6 & 92.14 & 0.045531 \\
\hline & 1,2-dibromoethane & DBRE12 & $\mathrm{C} 2 \mathrm{H} 4 \mathrm{Br} 2$ & 131.4 & 28.05 & 0.149542 \\
\hline & Chlorobenzene & CLBENZEN & $\mathrm{C} 6 \mathrm{H} 5 \mathrm{Cl}$ & 131.7 & 112.56 & 0.037272 \\
\hline & 1,2,3-trichloropropane & TCLPRP123 & $\mathrm{C} 3 \mathrm{H} 5 \mathrm{Cl} 3$ & 156.9 & 147.43 & 0.028456 \\
\hline & phenol & С6H5OH & C6H5OH & 181.8 & 94.11 & $\begin{array}{l}0.044577 \\
0 . * 3 *\end{array}$ \\
\hline & Hexachlorobutadiene & HXCL13BD & $\mathrm{C} 4 \mathrm{Cl} 6$ & 215.0 & 260.76 & 0.016089 \\
\hline \multirow{10}{*}{$\begin{array}{l}\text { Semi- } \\
\text { volatile }\end{array}$} & 1,2,4-trichlorobenzene & TCLBNZ124 & $\mathrm{C} 6 \mathrm{H} 3 \mathrm{Cl} 3$ & 213.0 & 181.45 & 0.023121 \\
\hline & Naphthalene & NAPHTHALEN & $\mathrm{C} 10 \mathrm{H} 8$ & 218.0 & 128.18 & 0.032731 \\
\hline & Hexachlorobenzene & CL6BENZEN & $\mathrm{C} 6 \mathrm{Cl} 6$ & 325.0 & 284.78 & 0.014731 \\
\hline & Pentachlorophenol & CL5PHENOL & $\mathrm{C} 6 \mathrm{Cl} 5 \mathrm{OH}$ & 309.4 & 266.34 & 0.015752 \\
\hline & Pyrene & PYRENE & $\mathrm{C} 16 \mathrm{H} 10$ & 393.0 & 202.26 & 0.020742 \\
\hline & $\begin{array}{l}\text { Bis(ethylhexyl)phthalate } \\
\text { (BEHP) }\end{array}$ & ВЕТНХРНTH2 & $\mathrm{C} 24 \mathrm{H} 38 \mathrm{O} 4$ & 384.0 & 390.57 & 0.010741 \\
\hline & Benzo(a)pyrene & BNZPYREN & $\mathrm{C} 20 \mathrm{H} 12$ & 311.0 & 252.32 & 0.016627 \\
\hline & 2-Chloronaphthalene & CLNAPHTH2 & $\mathrm{C} 10 \mathrm{H} 7 \mathrm{Cl}$ & 259.0 & 162.62 & 0.025798 \\
\hline & Dibenz[a,h]anthracene & DBANTHAH & $\mathrm{C} 22 \mathrm{H} 14$ & 524.0 & 278.36 & 0.015072 \\
\hline & Diethyl phthalate & DIETPHTHL & $\mathrm{C} 12 \mathrm{H} 14 \mathrm{O} 4$ & 294.0 & 222.24 & 0.018877 \\
\hline \multirow[t]{2}{*}{ Pesticide } & Aldrin & ALDRINE & $\mathrm{C} 12 \mathrm{H} 8 \mathrm{Cl} 6$ & 440.0 & 364.92 & 0.011497 \\
\hline & gamma-BHC (Lindane) & LINDANE & $\mathrm{C} 6 \mathrm{H} 6 \mathrm{Cl} 6$ & 323.4 & 290.83 & 0.014425 \\
\hline
\end{tabular}




\section{WSRC-TR-2002-00550, Rev. 0 \\ SRT-RPP-2002-00274}

\subsection{Expand the existing evaporator model to include the evaporator off-gas system}

The existing waste feed evaporator OLI model used in the earlier regulatory studies ${ }^{11}$ was expanded to include the off-gas system beyond the primary condenser and to include the ultrafiltration-caustic washing recycles after the evaporator. The off-gas system components included in the expanded OLI model were the seam ejectors, pressure control air valve, intercondenser, aftercondenser, condensate vessel, and demister. Some of the key features for each off-gas component are given next ${ }^{10}$. The expanded waste feed evaporator is described in detail in Appendix B.

Two steam ejectors are used to pull a necessary vacuum so that the liquid in the separator vessel (FEP-SEP$0001 \mathrm{~A} / \mathrm{B}$ ) will boil at $50{ }^{\circ} \mathrm{C}$. Both ejectors use superheated steam provided at a pressure of $109 \mathrm{psig}$ and a temperature of $173{ }^{\circ} \mathrm{C}$. Each steam ejector was modeled as a simple, adiabatic MIX node. The steam ejectors steam flow was determined from generic ejector performance curves with a Discharge/Suction Pressure compression ratio of 3 and an optimum area ratio of 50. Using a generic ejector performance curve from Perry's 5th p. 6-29, the entrainment at $90 \%$ efficiency was $0.5 \mathrm{lb}$ suction vapor $/ \mathrm{lb}$ steam. To match the given suction gas flow, $100 \mathrm{lb} / \mathrm{hr}$ of superheated steam was chosen. Actually less steam (about $80 \mathrm{lb} / \mathrm{hr}$ ) would be needed ideally but the larger amount was chosen since the actual ejector performance curve will not be as idealized.

Both the intercondenser and aftercondenser for the first and second steam ejectors were assumed to operate at $40{ }^{\circ} \mathrm{C}$. Each condenser was modeled in OLI as a simple, vapor-liquid equilibrium separator operating at a constant temperature. A nominal 5\% of operating pressure was used to represent the pressure drop in the condensers as suggested in vendor literature as well as other offgas equipment. It is assumed that the offgas exiting a condenser is saturated.

A demister (FEP-DMST-00001 A/B) removes entrained aerosols from the aftercondenser offgas vent. The concentrations of target organics remaining in the discharge vent fom this demister represent the air emission into the Process Vessel Vent System (PVVS). The demister was modeled in OLI as an adiabatic separator with $99 \%$ removal efficiency. This removal efficiency is based on SRS experience. The value can be changed to reflect vendor specifications. This removal efficiency was represented by a $0.01 \mathrm{~g}$ liquid/g vapor entrainment ratio in the OLI separator vessel.

The condensate produced in the primary condenser (FEP-COND-00001 A/B), intercondenser (FEP-COND$00002 \mathrm{~A} / \mathrm{B}$ ), and aftercondenser (FEP-COND-00003 A/B) is all gravity drained to the waste feed evaporator condensate vessel (FEP-VSL-00005). The condensate vessel was modeled in OLI as an isothermal MIX tank with a controlled air purge. A design basis air inleakage rate was used.

Control air for the vacuum system enters just upstream of the first steam ejector right after the primary condenser. The motive steam flow to each ejector was fixed at $100 \mathrm{lb} / \mathrm{hr}$ based on generic ejector curves and the suction gas flow entering the ejector. The control air flow was set at 2 times the estimated amount of air inleakage. In addition the control air was assumed to be $25 \%$ saturated. As a result, saturated off-gas exiting the primary condenser will become under saturated at the inlet of the first steam ejector. Since the waste feed evaporator OLI model describes a steady state operation only, there is no need to model controller actions and/or valve dynamics associated with the modulation of control air flow. An adiabatic MIX block was added to mix the primary condenser vent flow with the control air flow.

\subsection{Calculate partitioning of target organic compounds}

The expanded waste feed evaporator OLI model was run to predict how the target organic compounds partitioned among the vapor and liquid discharge streams of the offgas system. The model was run under the two conditions of normal operation of $1.22 \mathrm{~g} / \mathrm{ml}$ and abnormal operation of $1.35 \mathrm{~g} / \mathrm{ml}$ for the ultrafiltration filtrate stream (UF1 Filtrate). Figure 1 shows the mass balance of the waste feed evaporator offgas run for the ultrafiltrate density of $1.22 \mathrm{~g} / \mathrm{ml}$. Table VII shows the organic feed flows of the evaporator waste feed and recycle streams for the ultrafiltrate density of 1.22. Table VIII shows the 
partitioning of the feed organics for the ultrafiltrate density of $1.22 \mathrm{~g} / \mathrm{ml}$. Figure 2 shows the mass balance of the waste feed evaporator offgas run for the ultrafiltrate density of $1.35 \mathrm{~g} / \mathrm{ml}$. Table IX shows the organic feed flows of the evaporator waste feed and recycle streams for the ultrafiltrate density of $1.35 \mathrm{~g} / \mathrm{ml}$. Table $\mathrm{X}$ shows the partitioning of the feed organics for the ultrafiltrate density of $1.35 \mathrm{~g} / \mathrm{ml}$. One page summary schematics of these two offgas run results are shown in Appendix A. Also in Appendix A are complete run outputs for all species in the OLI model.

\subsection{Validate predicted partitioning of target organics against pilot data}

A pilot evaporator will be built and then the OLI model results will be compared to the measured data. Of primary importance to model validation are the samples of separator vessel liquid, primary condenser liquid, primary condenser vent, demister vent, and condensate vessel liquid. Some of these liquids may or may not be available for analysis. 


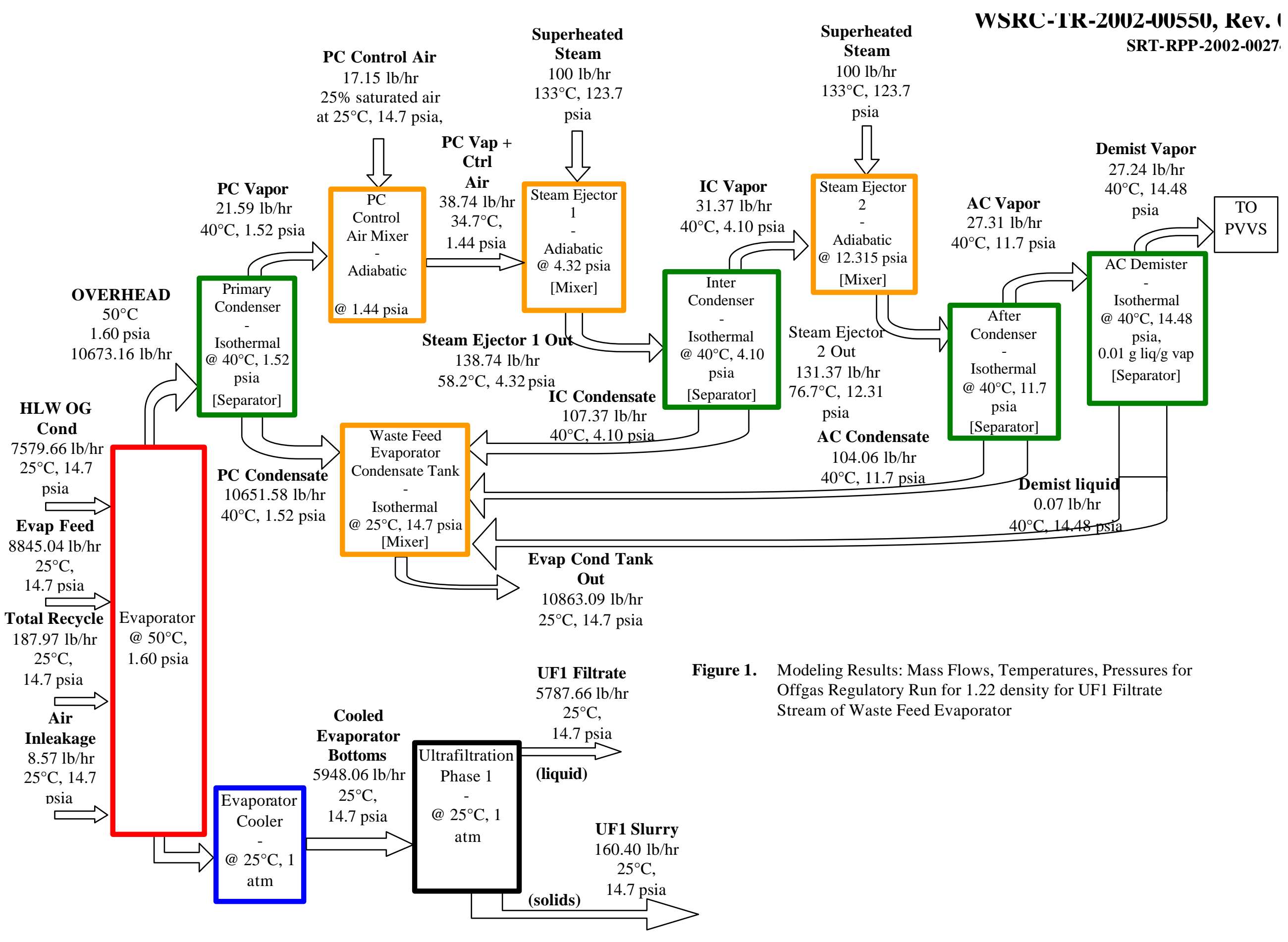


Table VII. Organic Feed Flows for Offgas Regulatory Modeling Run UF1 Filtrate 1.22 density ${ }^{8}$

\begin{tabular}{|c|c|c|}
\hline Stream & Total Recycle & Evap Feed \\
\hline Phase & Mixed & Mixed \\
\hline Temperature, $\mathrm{C}$ & 25 & 25 \\
\hline Pressure, atm & 1 & 1 \\
\hline $\mathrm{pH}$ & 13.4748 & 13.0705 \\
\hline Total $\mathrm{mol} / \mathrm{hr}$ & 4402.02 & 197463 \\
\hline Mass Flow Units & $\mathrm{g} / \mathrm{hr}$ & $\mathrm{g} / \mathrm{hr}$ \\
\hline Aldrin & $1.568 \mathrm{E}-04$ & $4.195 \mathrm{E}+00$ \\
\hline Benzene & $7.739 \mathrm{E}-07$ & $4.194 \mathrm{E}+00$ \\
\hline Bis(2-ethylhexyl)phthalate (BEHP) & $1.971 \mathrm{E}-03$ & $4.193 \mathrm{E}+00$ \\
\hline Benzo(a)pyrene (BaP) & $9.236 \mathrm{E}-06$ & $4.181 \mathrm{E}+00$ \\
\hline Phenol & $2.747 \mathrm{E}-02$ & $4.196 \mathrm{E}+00$ \\
\hline Pentachlorophenol & $2.372 \mathrm{E}-03$ & $4.196 \mathrm{E}+00$ \\
\hline Hexachlorobenzene & $2.205 \mathrm{E}-05$ & $4.188 \mathrm{E}+00$ \\
\hline Chlorobenzene & $1.660 \mathrm{E}-06$ & $4.190 \mathrm{E}+00$ \\
\hline 2-Chloronaphthalene & $4.459 \mathrm{E}-06$ & $4.194 \mathrm{E}+00$ \\
\hline Dibenz[a,h]anthracene & $1.102 \mathrm{E}-03$ & $4.191 \mathrm{E}+00$ \\
\hline 1,2-dibromoethane & $3.989 \mathrm{E}-05$ & $2.806 \mathrm{E}+01$ \\
\hline Diethyl phthalate & $5.992 \mathrm{E}-03$ & $4.202 \mathrm{E}+00$ \\
\hline Mercury Chloride & $0.000 \mathrm{E}+00$ & $0.000 \mathrm{E}+00$ \\
\hline Mercury (sol) & $3.801 \mathrm{E}-06$ & $0.000 \mathrm{E}+00$ \\
\hline Mercuric Oxide (insol) & $9.851 \mathrm{E}-01$ & $1.361 \mathrm{E}+02$ \\
\hline Hexachlorobutadiene & $3.470 \mathrm{E}-08$ & $4.200 \mathrm{E}+00$ \\
\hline gamma-BHC (Lindane) & 9.689E-05 & $4.209 \mathrm{E}+00$ \\
\hline 4-methyl-2-pentanone (MIBK) & $1.850 \mathrm{E}-05$ & $4.197 \mathrm{E}+00$ \\
\hline Naphthalene & $4.906 \mathrm{E}-06$ & $4.188 \mathrm{E}+00$ \\
\hline Pyrene & $1.524 \mathrm{E}-04$ & $4.202 \mathrm{E}+00$ \\
\hline 1,2,4-trichlorobenzene & $1.035 \mathrm{E}-06$ & $4.193 \mathrm{E}+00$ \\
\hline 1,2,3-trichloropropane & $7.301 \mathrm{E}-06$ & $4.198 \mathrm{E}+00$ \\
\hline Toluene & $6.433 \mathrm{E}-07$ & $4.194 \mathrm{E}+00$ \\
\hline Total $\mathrm{g} / \mathrm{hr}$ & 85260.4 & $4.01 \mathrm{E}+06$ \\
\hline Volume, L/hr & 79.456 & 3550.76 \\
\hline Enthalpy, cal/hr & $-3.07 \mathrm{E}+08$ & $-1.40 \mathrm{E}+10$ \\
\hline \multicolumn{3}{|l|}{ Vapor fraction } \\
\hline Solid fraction & $2.78 \mathrm{E}-04$ & $2.24 \mathrm{E}-04$ \\
\hline Organic fraction & $9.59 \mathrm{E}-10$ & 4.89E-07 \\
\hline Osmotic Pres, atm & 60.765 & 90.7109 \\
\hline \multicolumn{3}{|l|}{ Redox Pot, volts } \\
\hline E-Con, 1/ohm-cm & 0.123482 & 0.137767 \\
\hline E-Con, cm2/ohm-mol & 48.9223 & 68.3032 \\
\hline Abs Visc, cP & 1.15 & 1.49431 \\
\hline Rel Visc & 1.29109 & 1.67765 \\
\hline Ionic Strength & 1.59552 & 3.06198 \\
\hline
\end{tabular}


WSRC-'IR-2002-00550, Rev. I SRT-RPP-2002-0027

Table VIII. Organic Partitioning In Terms Of Waste Feed and Recycle Streams for Offgas Regulatory Modeling Run UF1 Filtrate 1.22 Density

\begin{tabular}{|c|c|c|c|c|c|c|c|c|c|c|c|c|c|}
\hline Stream & Overhead & $\begin{array}{c}\text { Cooled } \\
\text { Evap Bott }\end{array}$ & $\begin{array}{c}\text { UF1 } \\
\text { Filtrate }\end{array}$ & $\begin{array}{c}\text { UF1 } \\
\text { Slurry }\end{array}$ & PC Vapor & $\begin{array}{c}\mathrm{PC} \\
\text { Condensate }\end{array}$ & IC Vapor & $\begin{array}{c}\text { IC } \\
\text { Condensate }\end{array}$ & $\begin{array}{c}\text { AC } \\
\text { Vapor }\end{array}$ & $\begin{array}{c}\mathrm{AC} \\
\text { Condensate }\end{array}$ & $\begin{array}{l}\text { Demist } \\
\text { Vapor }\end{array}$ & $\begin{array}{l}\text { Demist } \\
\text { Liquid }\end{array}$ & $\begin{array}{c}\text { Evap } \\
\text { Cond Tk } \\
\text { Out }\end{array}$ \\
\hline Aldrin & $85.66 \%$ & $14.34 \%$ & $14.03 \%$ & $0.31 \%$ & $0.22 \%$ & $85.44 \%$ & $0.07 \%$ & $0.15 \%$ & $0.01 \%$ & $0.06 \%$ & $0.01 \%$ & $0.00 \%$ & $85.65 \%$ \\
\hline Benzene & $100.00 \%$ & $0.00 \%$ & $0.00 \%$ & $0.00 \%$ & $90.23 \%$ & $9.76 \%$ & $90.01 \%$ & $0.22 \%$ & $89.26 \%$ & $0.75 \%$ & $89.26 \%$ & $0.00 \%$ & $10.74 \%$ \\
\hline Bis(2-ethylhexyl)phthalate (BEHP) & $42.70 \%$ & $57.30 \%$ & $56.06 \%$ & $1.24 \%$ & $0.01 \%$ & $42.69 \%$ & $0.00 \%$ & $0.01 \%$ & $0.00 \%$ & $0.00 \%$ & $0.00 \%$ & $0.00 \%$ & $42.70 \%$ \\
\hline Benzo(a)pyrene (BaP) & $0.00 \%$ & $100.00 \%$ & $0.05 \%$ & $99.95 \%$ & $0.00 \%$ & $0.00 \%$ & $0.00 \%$ & $0.00 \%$ & $0.00 \%$ & $0.00 \%$ & $0.00 \%$ & $0.00 \%$ & $0.00 \%$ \\
\hline Phenol & $0.15 \%$ & $99.85 \%$ & $97.68 \%$ & $2.17 \%$ & $0.00 \%$ & $0.15 \%$ & $0.00 \%$ & $0.00 \%$ & $0.00 \%$ & $0.00 \%$ & $0.00 \%$ & $0.00 \%$ & $0.15 \%$ \\
\hline Pentachlorophenol & $90.84 \%$ & $9.16 \%$ & $8.97 \%$ & $0.20 \%$ & $0.14 \%$ & $90.69 \%$ & $0.01 \%$ & $0.14 \%$ & $0.00 \%$ & $0.01 \%$ & $0.00 \%$ & $0.00 \%$ & $90.83 \%$ \\
\hline Hexachlorobenzene & $0.00 \%$ & $100.00 \%$ & $0.11 \%$ & $99.89 \%$ & $0.00 \%$ & $0.00 \%$ & $0.00 \%$ & $0.00 \%$ & $0.00 \%$ & $0.00 \%$ & $0.00 \%$ & $0.00 \%$ & $0.00 \%$ \\
\hline Chlorobenzene & $99.99 \%$ & $0.01 \%$ & $0.01 \%$ & $0.00 \%$ & $82.59 \%$ & $17.40 \%$ & $82.19 \%$ & $0.40 \%$ & $80.87 \%$ & $1.32 \%$ & $80.87 \%$ & $0.00 \%$ & $19.13 \%$ \\
\hline 2-Chloronaphthalene & $99.98 \%$ & $0.02 \%$ & $0.02 \%$ & $0.00 \%$ & $50.84 \%$ & $49.14 \%$ & $49.76 \%$ & $1.08 \%$ & $46.38 \%$ & $3.39 \%$ & $46.37 \%$ & $0.01 \%$ & $53.61 \%$ \\
\hline Dibenz[a,h]anthracene & $0.22 \%$ & $99.78 \%$ & $97.61 \%$ & $2.16 \%$ & $0.00 \%$ & $0.22 \%$ & $0.00 \%$ & $0.00 \%$ & $0.00 \%$ & $0.00 \%$ & $0.00 \%$ & $0.00 \%$ & $0.22 \%$ \\
\hline 1,2-dibromoethane & $99.98 \%$ & $0.02 \%$ & $0.02 \%$ & $0.00 \%$ & $53.16 \%$ & $46.82 \%$ & $52.10 \%$ & $1.06 \%$ & $48.76 \%$ & $3.34 \%$ & $48.76 \%$ & $0.00 \%$ & $51.22 \%$ \\
\hline Diethyl phthalate & $78.05 \%$ & $21.94 \%$ & $21.47 \%$ & $0.48 \%$ & $0.04 \%$ & $78.02 \%$ & $0.00 \%$ & $0.04 \%$ & $0.00 \%$ & $0.00 \%$ & $0.00 \%$ & $0.00 \%$ & $78.06 \%$ \\
\hline Mercury Chloride & $4.60 \%$ & $96.40 \%$ & $0.00 \%$ & $0.00 \%$ & $0.00 \%$ & $0.00 \%$ & $0.00 \%$ & $0.00 \%$ & $0.00 \%$ & $0.00 \%$ & $0.00 \%$ & $0.00 \%$ & $0.00 \%$ \\
\hline Mercury (sol) & $100.00 \%$ & $0.00 \%$ & $0.00 \%$ & $0.00 \%$ & $12.26 \%$ & $87.73 \%$ & $5.37 \%$ & $6.90 \%$ & $1.53 \%$ & $3.84 \%$ & $1.22 \%$ & $0.32 \%$ & $98.78 \%$ \\
\hline Mercuric Oxide (insol) & $0.00 \%$ & $100.00 \%$ & $56.91 \%$ & $43.09 \%$ & $0.00 \%$ & $0.00 \%$ & $0.00 \%$ & $0.00 \%$ & $0.00 \%$ & $0.00 \%$ & $0.00 \%$ & $0.00 \%$ & $0.00 \%$ \\
\hline Hexachlorobutadiene & $100.00 \%$ & $0.00 \%$ & $0.00 \%$ & $0.00 \%$ & $99.28 \%$ & $0.72 \%$ & $99.27 \%$ & $0.01 \%$ & $99.23 \%$ & $0.04 \%$ & $99.23 \%$ & $0.00 \%$ & $0.77 \%$ \\
\hline gamma-BHC (Lindane) & $99.56 \%$ & $0.44 \%$ & $0.43 \%$ & $0.01 \%$ & $5.93 \%$ & $93.64 \%$ & $4.43 \%$ & $1.49 \%$ & $2.08 \%$ & $2.35 \%$ & $2.08 \%$ & $0.00 \%$ & $97.49 \%$ \\
\hline 4-methyl-2-pentanone (MIBK) & $99.93 \%$ & $0.07 \%$ & $0.07 \%$ & $0.00 \%$ & $23.37 \%$ & $76.56 \%$ & $21.73 \%$ & $1.64 \%$ & $17.33 \%$ & $4.41 \%$ & $17.32 \%$ & $0.00 \%$ & $82.61 \%$ \\
\hline Naphthalene & $99.98 \%$ & $0.02 \%$ & $0.02 \%$ & $0.00 \%$ & $60.12 \%$ & $39.86 \%$ & $59.22 \%$ & $0.90 \%$ & $56.35 \%$ & $2.87 \%$ & $56.34 \%$ & $0.00 \%$ & $43.64 \%$ \\
\hline Pyrene & $96.12 \%$ & $3.88 \%$ & $3.79 \%$ & $0.08 \%$ & $1.06 \%$ & $95.06 \%$ & $0.56 \%$ & $0.50 \%$ & $0.14 \%$ & $0.43 \%$ & $0.13 \%$ & $0.01 \%$ & $96.00 \%$ \\
\hline 1,2,4-trichlorobenzene & $100.00 \%$ & $0.00 \%$ & $0.00 \%$ & $0.00 \%$ & $83.04 \%$ & $16.96 \%$ & $82.66 \%$ & $0.38 \%$ & $81.40 \%$ & $1.26 \%$ & $81.40 \%$ & $0.00 \%$ & $18.60 \%$ \\
\hline 1,2,3-trichloropropane & $99.97 \%$ & $0.03 \%$ & $0.03 \%$ & $0.00 \%$ & $44.56 \%$ & $55.42 \%$ & $43.32 \%$ & $1.24 \%$ & $39.51 \%$ & $3.81 \%$ & $39.51 \%$ & $0.00 \%$ & $60.47 \%$ \\
\hline Toluene & $100.00 \%$ & $0.00 \%$ & $0.00 \%$ & $0.00 \%$ & $91.37 \%$ & $8.63 \%$ & $91.17 \%$ & $0.20 \%$ & $90.50 \%$ & $0.66 \%$ & $90.50 \%$ & $0.00 \%$ & $9.49 \%$ \\
\hline
\end{tabular}




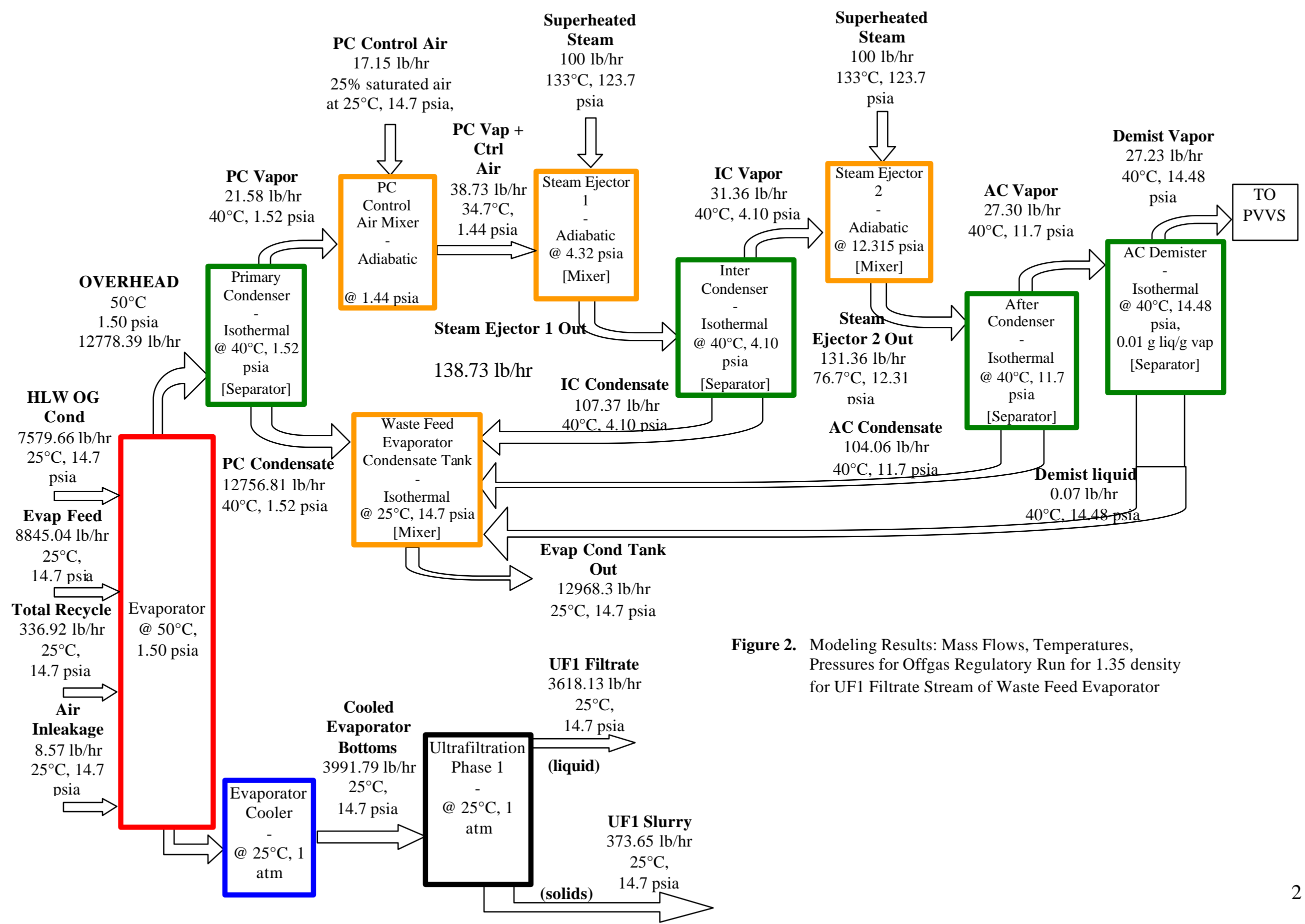


Table IX. Organic Feed Flows for Offgas Regulatory Modeling Run UF1 Filtrate 1.35 density ${ }^{\mathbf{8}}$

\begin{tabular}{|c|c|c|}
\hline Stream & Total Recycle & Evap Feed \\
\hline Phase & Mixed & Mixed \\
\hline Temperature, $\mathrm{C}$ & 25 & 25 \\
\hline Pressure, atm & 1 & 1 \\
\hline $\mathrm{pH}$ & 12.8607 & 13.0705 \\
\hline Total mol/hr & 7912.79 & 197463 \\
\hline Flow Units & $\mathrm{g} / \mathrm{hr}$ & $\mathrm{g} / \mathrm{hr}$ \\
\hline Aldrin & $7.204 \mathrm{E}-05$ & $4.195 \mathrm{E}+00$ \\
\hline Benzene & $3.537 \mathrm{E}-07$ & $4.194 \mathrm{E}+00$ \\
\hline Bis(2-ethylhexyl)phthalate (BEHP) & $9.539 \mathrm{E}-04$ & $4.193 \mathrm{E}+00$ \\
\hline Benzo(a)pyrene $(\mathrm{BaP})$ & $6.746 \mathrm{E}-06$ & $4.181 \mathrm{E}+00$ \\
\hline Phenol & $5.364 \mathrm{E}-02$ & $4.196 \mathrm{E}+00$ \\
\hline Pentachlorophenol & $1.161 \mathrm{E}-03$ & $4.196 \mathrm{E}+00$ \\
\hline Hexachlorobenzene & $1.610 \mathrm{E}-05$ & $4.188 \mathrm{E}+00$ \\
\hline Chlorobenzene & $7.586 \mathrm{E}-07$ & $4.190 \mathrm{E}+00$ \\
\hline 2-Chloronaphthalene & $2.038 \mathrm{E}-06$ & $4.194 \mathrm{E}+00$ \\
\hline Dibenz[a,h]anthracene & $5.199 \mathrm{E}-04$ & $4.191 \mathrm{E}+00$ \\
\hline 1,2-dibromoethane & $1.823 \mathrm{E}-05$ & $2.806 \mathrm{E}+01$ \\
\hline Diethyl phthalate & $3.287 \mathrm{E}-03$ & $4.202 \mathrm{E}+00$ \\
\hline \multicolumn{3}{|l|}{ Mercury Chloride } \\
\hline Mercury (sol) & $1.744 \mathrm{E}-06$ & \\
\hline Mercuric Oxide (insol) & $1.534 \mathrm{E}+00$ & $1.361 \mathrm{E}+02$ \\
\hline Hexachlorobutadiene & $1.590 \mathrm{E}-08$ & $4.200 \mathrm{E}+00$ \\
\hline gamma-BHC (Lindane) & $4.442 \mathrm{E}-05$ & $4.209 \mathrm{E}+00$ \\
\hline 4-methyl-2-pentanone (MIBK) & $8.460 \mathrm{E}-06$ & $4.197 \mathrm{E}+00$ \\
\hline Naphthalene & $2.243 \mathrm{E}-06$ & $4.188 \mathrm{E}+00$ \\
\hline Pyrene & $6.996 \mathrm{E}-05$ & $4.202 \mathrm{E}+00$ \\
\hline 1,2,4-trichlorobenzene & $4.735 \mathrm{E}-07$ & $4.193 \mathrm{E}+00$ \\
\hline 1,2,3-trichloropropane & $3.338 \mathrm{E}-06$ & $4.198 \mathrm{E}+00$ \\
\hline Toluene & $2.940 \mathrm{E}-07$ & $4.194 \mathrm{E}+00$ \\
\hline Total $\mathrm{g} / \mathrm{hr}$ & 152823 & $4.01 \mathrm{E}+06$ \\
\hline Volume, L/hr & 143.624 & 3550.76 \\
\hline Enthalpy, cal/hr & $-5.51 \mathrm{E}+08$ & $-1.40 \mathrm{E}+10$ \\
\hline \multicolumn{3}{|l|}{ Vapor fraction } \\
\hline Solid fraction & $1.71 \mathrm{E}-04$ & 2.24E-04 \\
\hline Organic fraction & $2.21 \mathrm{E}-10$ & $4.89 \mathrm{E}-07$ \\
\hline Osmotic Pres, atm & 48.2193 & 90.7109 \\
\hline \multicolumn{3}{|l|}{ Redox Pot, volts } \\
\hline E-Con, 1/ohm-cm & 0.0918338 & 0.137767 \\
\hline E-Con, cm2/ohm-mol & 43.1466 & 68.3032 \\
\hline Abs Visc, cP & 1.06321 & 1.49431 \\
\hline Rel Visc & 1.19366 & 1.67765 \\
\hline Ionic Strength & 1.37611 & 3.06198 \\
\hline
\end{tabular}


WSRC-'IR-2002-00550, Rev. I SRT-RPP-2002-0027

Table X. Organic Partitioning In Terms Of Waste Feed and Recycle Streams for Offgas Regulatory Modeling Run UF1 Filtrate 1.35 Density ${ }^{8}$

\begin{tabular}{|c|c|c|c|c|c|c|c|c|c|c|c|c|c|}
\hline Stream & Overhead & $\begin{array}{c}\text { Cooled } \\
\text { Evap Bott }\end{array}$ & $\begin{array}{c}\text { UF1 } \\
\text { Filtrate }\end{array}$ & $\begin{array}{c}\text { UF1 } \\
\text { Slurry }\end{array}$ & PC Vapor & \begin{tabular}{|c|}
$\mathrm{PC}$ \\
Condensate
\end{tabular} & IC Vapor & $\begin{array}{c}\text { IC } \\
\text { Condensate }\end{array}$ & $\begin{array}{c}\text { AC } \\
\text { Vapor }\end{array}$ & $\begin{array}{c}\mathrm{AC} \\
\text { Condensate }\end{array}$ & $\begin{array}{l}\text { Demist } \\
\text { Vapor }\end{array}$ & $\begin{array}{l}\text { Demist } \\
\text { Liquid }\end{array}$ & $\begin{array}{c}\text { Evap } \\
\text { Cond Tk } \\
\text { Out }\end{array}$ \\
\hline Aldrin & $89.58 \%$ & $10.42 \%$ & $9.63 \%$ & $0.80 \%$ & $0.22 \%$ & $89.36 \%$ & $0.08 \%$ & $0.14 \%$ & $0.01 \%$ & $0.07 \%$ & $0.01 \%$ & $0.00 \%$ & $89.57 \%$ \\
\hline Benzene & $100.00 \%$ & $0.00 \%$ & $0.00 \%$ & $0.00 \%$ & $88.52 \%$ & $11.47 \%$ & $88.30 \%$ & $0.22 \%$ & $87.57 \%$ & $0.74 \%$ & $87.57 \%$ & $0.00 \%$ & $12.43 \%$ \\
\hline Bis(2-ethylhexyl)phthalate (BEHP) & $53.47 \%$ & $46.53 \%$ & $42.98 \%$ & $3.55 \%$ & $0.01 \%$ & $53.46 \%$ & $0.00 \%$ & $0.01 \%$ & $0.00 \%$ & $0.00 \%$ & $0.00 \%$ & $0.00 \%$ & $53.47 \%$ \\
\hline Benzo(a)pyrene (BaP) & $0.00 \%$ & $100.00 \%$ & $0.01 \%$ & $99.99 \%$ & $0.00 \%$ & $0.00 \%$ & $0.00 \%$ & $0.00 \%$ & $0.00 \%$ & $0.00 \%$ & $0.00 \%$ & $0.00 \%$ & $0.00 \%$ \\
\hline Phenol & $0.11 \%$ & $99.89 \%$ & $92.27 \%$ & $7.62 \%$ & $0.00 \%$ & $0.11 \%$ & $0.00 \%$ & $0.00 \%$ & $0.00 \%$ & $0.00 \%$ & $0.00 \%$ & $0.00 \%$ & $0.11 \%$ \\
\hline Pentachlorophenol & $97.37 \%$ & $2.62 \%$ & $2.42 \%$ & $0.20 \%$ & $0.13 \%$ & $97.24 \%$ & $0.01 \%$ & $0.12 \%$ & $0.00 \%$ & $0.01 \%$ & $0.00 \%$ & $0.00 \%$ & $97.37 \%$ \\
\hline Hexachlorobenzene & $0.00 \%$ & $100.00 \%$ & $0.03 \%$ & $99.97 \%$ & $0.00 \%$ & $0.00 \%$ & $0.00 \%$ & $0.00 \%$ & $0.00 \%$ & $0.00 \%$ & $0.00 \%$ & $0.00 \%$ & $0.00 \%$ \\
\hline Chlorobenzene & $100.00 \%$ & $0.00 \%$ & $0.00 \%$ & $0.00 \%$ & $79.84 \%$ & $20.16 \%$ & $79.46 \%$ & $0.38 \%$ & $78.18 \%$ & $1.28 \%$ & $78.18 \%$ & $0.00 \%$ & $21.82 \%$ \\
\hline 2-Chloronaphthalene & $99.99 \%$ & $0.01 \%$ & $0.01 \%$ & $0.00 \%$ & $46.50 \%$ & $53.49 \%$ & $45.52 \%$ & $0.98 \%$ & $42.43 \%$ & $3.09 \%$ & $42.42 \%$ & $0.00 \%$ & $57.57 \%$ \\
\hline Dibenz[a,h]anthracene & $0.30 \%$ & $99.70 \%$ & $92.09 \%$ & $7.61 \%$ & $0.00 \%$ & $0.30 \%$ & $0.00 \%$ & $0.00 \%$ & $0.00 \%$ & $0.00 \%$ & $0.00 \%$ & $0.00 \%$ & $0.30 \%$ \\
\hline 1,2-dibromoethane & $100.00 \%$ & $0.01 \%$ & $0.00 \%$ & $0.00 \%$ & $48.65 \%$ & $51.34 \%$ & $47.69 \%$ & $0.97 \%$ & $44.63 \%$ & $3.06 \%$ & $44.63 \%$ & $0.00 \%$ & $55.36 \%$ \\
\hline Diethyl phthalate & $93.72 \%$ & $6.28 \%$ & $5.80 \%$ & $0.48 \%$ & $0.04 \%$ & $93.69 \%$ & $0.00 \%$ & $0.04 \%$ & $0.00 \%$ & $0.00 \%$ & $0.00 \%$ & $0.00 \%$ & $93.72 \%$ \\
\hline Mercury Chloride & $4.60 \%$ & $96.40 \%$ & $0.00 \%$ & $0.00 \%$ & $0.00 \%$ & $0.00 \%$ & $0.00 \%$ & $0.00 \%$ & $0.00 \%$ & $0.00 \%$ & $0.00 \%$ & $0.00 \%$ & $0.00 \%$ \\
\hline Mercury (sol) & $100.00 \%$ & $0.00 \%$ & $0.00 \%$ & $0.00 \%$ & $12.21 \%$ & $87.79 \%$ & $5.35 \%$ & $6.87 \%$ & $1.53 \%$ & $3.82 \%$ & $1.21 \%$ & $0.32 \%$ & $98.79 \%$ \\
\hline Mercuric Oxide (insol) & $0.00 \%$ & $100.00 \%$ & $26.08 \%$ & $73.92 \%$ & $0.00 \%$ & $0.00 \%$ & $0.00 \%$ & $0.00 \%$ & $0.00 \%$ & $0.00 \%$ & $0.00 \%$ & $0.00 \%$ & $0.00 \%$ \\
\hline Hexachlorobutadiene & $100.00 \%$ & $0.00 \%$ & $0.00 \%$ & $0.00 \%$ & $99.17 \%$ & $0.83 \%$ & $99.16 \%$ & $0.01 \%$ & $99.12 \%$ & $0.04 \%$ & $99.12 \%$ & $0.00 \%$ & $0.88 \%$ \\
\hline gamma-BHC (Lindane) & $99.85 \%$ & $0.15 \%$ & $0.14 \%$ & $0.01 \%$ & $5.08 \%$ & $94.77 \%$ & $3.81 \%$ & $1.28 \%$ & $1.79 \%$ & $2.02 \%$ & $1.78 \%$ & $0.00 \%$ & $98.07 \%$ \\
\hline 4-methyl-2-pentanone (MIBK) & $99.98 \%$ & $0.02 \%$ & $0.01 \%$ & $0.00 \%$ & $20.30 \%$ & $79.68 \%$ & $18.88 \%$ & $1.42 \%$ & $15.05 \%$ & $3.83 \%$ & $15.05 \%$ & $0.00 \%$ & $84.94 \%$ \\
\hline Naphthalene & $100.00 \%$ & $0.00 \%$ & $0.00 \%$ & $0.00 \%$ & $55.77 \%$ & $44.23 \%$ & $54.94 \%$ & $0.83 \%$ & $52.27 \%$ & $2.67 \%$ & $52.27 \%$ & $0.00 \%$ & $47.73 \%$ \\
\hline Pyrene & $97.35 \%$ & $2.65 \%$ & $2.45 \%$ & $0.20 \%$ & $1.03 \%$ & $96.31 \%$ & $0.56 \%$ & $0.47 \%$ & $0.14 \%$ & $0.42 \%$ & $0.13 \%$ & $0.01 \%$ & $97.21 \%$ \\
\hline 1,2,4-trichlorobenzene & $100.00 \%$ & $0.00 \%$ & $0.00 \%$ & $0.00 \%$ & $80.43 \%$ & $19.57 \%$ & $80.06 \%$ & $0.37 \%$ & $78.85 \%$ & $1.22 \%$ & $78.84 \%$ & $0.00 \%$ & $21.16 \%$ \\
\hline 1,2,3-trichloropropane & $99.99 \%$ & $0.01 \%$ & $0.01 \%$ & $0.00 \%$ & $40.16 \%$ & $59.83 \%$ & $39.05 \%$ & $1.12 \%$ & $35.61 \%$ & $3.43 \%$ & $35.61 \%$ & $0.00 \%$ & $64.39 \%$ \\
\hline Toluene & $100.00 \%$ & $0.00 \%$ & $0.00 \%$ & $0.00 \%$ & $89.83 \%$ & $10.17 \%$ & $89.64 \%$ & $0.19 \%$ & $88.99 \%$ & $0.65 \%$ & $88.99 \%$ & $0.00 \%$ & $11.01 \%$ \\
\hline
\end{tabular}




\subsection{DISCUSSION OF RESULTS}

The source of organics in the waste feed evaporator offgas OLI model are the waste feed and total recycle streams from the caustic washing and leaching ultrafiltration steps. In order to get the total recycle stream composition and flow rate, a five stage waste feed evaporator OLI model was used. This 5-stage waste feed evaporator OLI model is described in detail in Appendix A. The resulting total recycle stream composition and the waste feed composition for the nominal offgas case with ultrafiltrate density of 1.22 is shown in Table XII and the abnormal case with ultrafiltrate density of 1.35 is shown in Table XIII. Also in these tables are the organic and mercury partitioning of each offgas stream in relation to the total input of organics in the waste feed and total recycle streams.

The normal $1.22 \mathrm{~g} / \mathrm{ml}$ ultrafiltrate density case differs from the abnormal $1.35 \mathrm{~g} / \mathrm{ml}$ density case primarily in the amount of water being evaporated. In the abnormal case $20 \%$ more water is evaporated into the evaporator overhead to meet the higher density requirement for the ultrafiltrate stream. This higher boil-off rate in turn impacts the organic partitioning. For the volatiles (Benzene, 4methyl-2-pentanone (MIBK), Toluene, 1,2-dibromoethane, Chlorobenzene, 1,2,3-trichloropropane, Phenol, Hexachlorobutadiene), the percent with respect to the total evaporator feed (including recycles) going into the evaporator overhead for the normal and abnormal case are about the same. For the semi-volatiles (1,2,4-trichlorobenzene, Naphthalene, Hexachlorobenzene, Pentachlorophenol, Pyrene, Bis(ethylhexyl)phthalate (BEHP), Benzo(a)pyrene, 2-Chloronaphthalene, Dibenz[a,h]anthracene, Diethyl phthalate), the Pentachlorophenol, Bis(2-ethylhexyl)phthalate (BEHP), and Diethyl phthalate for the abnormal case show 7\%, 11\%, and $16 \%$ more of these components with respect to the total evaporator feed (including recycles) going out the evaporator overhead than the normal case. As a result, $7 \%, 11 \%$, and $16 \%$ less of these components end up in the cooled evaporator bottoms and $7 \%, 11 \%$, and $16 \%$ more of the components end up in the primary condenser condensate. The pesticides (Aldrin, gamma-BHC (Lindane)) behave basically the same for the normal and abnormal cases. Based on these OLI results some of semi-volatile components (Pentachlorophenol, Bis(2-ethylhexyl)phthalate (BEHP), and Diethyl phthalate) are more susceptible to the amount of water being boiled off in the evaporator. Other than these specific semi-volatiles, the organic partitioning remains about the same or within $5 \%$ between the two cases.

The compounds phenol, hexachlorobenzene, and benzo(a)pyrene despite their classification as volatile or semi-volatile come out the cooled evaporator bottoms. This result is because OLI predicts a high solubility of each of these compounds in water. This solubility inhibits the carry over of these compounds in the evaporator overhead stream. This behavior has been observed in earlier OLI runs and experiments ${ }^{4}$.

This work does not reflect the true conditions of mercury in the waste feed evaporator. For this reason it is strongly suggested that the mercury calculations not be used in the evaporator engineering calculations for design. SRTC will be conducting experiments to attempt to provide a mercury mass balance across the waste feed evaporator. ${ }^{6}$ This new work will be better suited for mercury analysis and engineering design calculations.

The mercury partitioning based on the information available was straightforward in the OLI modeling. Whatever elemental mercury enters the evaporator goes out the overhead. About $5 \%$ of the mercuric chloride in the evaporator feed goes out the evaporator overhead. No other mercury species were observed in the evaporator overhead. Any insoluble mercury entering the evaporator goes out the bottoms. The mercury redox only defines how much of the insoluble mercury entering the evaporator is converted to elemental mercury. The assumption of $50 \%$ reduction of mercuric oxide to mercury was purely arbitrary to show the effects of redox on the system. If a $0 \%$ mercury reduction is assumed then none of the insoluble mercury in the feed is reduced. The amount of elemental mercury in the feed may be higher than true values. Regardless of the amount present, any elemental mercury in the feed is predicted by OLI to go out the top of the evaporator. SRTC is planning some experiments to verify the partitioning of mercury in an evaporator setup versus OLI predictions. Only about $0.8 \%$ of the total mercury in the waste feed returns through the total recycle stream as soluble mercury (mercuric hydroxide and hydroxide ion) from the 
ultrafiltration/caustic washing/leaching step to the evaporator. There were no odd mercury compounds that OLI predicted forming.

The current offgas OLI modeling was compared with the prior work of Hiroshi ${ }^{11}$ as an internal check. In order to make this comparison, certain model streams from Hiroshi's work had to be equated with the new offgas model streams: Hiroshi's primary condenser condensate equates to the new primary condenser condensate, Hirohsi's cooled evaporator bottoms equates to the new evaporator cooled bottoms, and Hiroshi's primary condenser vapor equates to the new primary condenser vapor. Even with this stream match up there were several differences between the Hiroshi's runs and FEP runs as shown in Table XI.

Table XI. Comparison of Hiroshi and FEP Modeling Runs

\begin{tabular}{|c|c|c|}
\hline Item & Hiroshi Runs & FEP Runs \\
\hline Waste Feed Type & $\begin{array}{c}\text { RPP AN-107 Envelope C- } \\
\text { simulant }\end{array}$ & RPP AZ-102 Envelope B \\
\hline Waste Feed Volume [L/hr] & 0.06 & 3551 \\
\hline SBS HLW Recycle Feed [L/hr] & None & 3405 \\
\hline $1^{\text {st }} / 2^{\text {nd }}$ Caustic Wash-Leach Recycle \\
Feed [L/hr] & None & 79 \\
\hline Waste Feed Na molarity & 5.5 & 2.65 \\
\hline Evaporator Feed Na Molarity & 5.5 & $1.56 \mathrm{E}-4$ \\
\hline Evaporator Conc. Na mo larity & 8 & 4.5 \\
\hline Waste Feed Vol/ Evap Conc Vol & 1.4 & 1.58 \\
\hline Evap Feed Vol/ Evap Conc Vol & 1.4 & 29100 \\
\hline Evap Conc Density & 1.35 & 1.22 \\
\hline
\end{tabular}

The primary difference between the two runs is that the FEP evaporator concentrated its feed more than Hiroshi evaporator and the waste feed evaporator added more water to the total evaporator feed via the SBS high level waste recycle. The physical structure of the FEP offgas regulatory OLI model is different than Hiroshi's model (REG_C). The FEP offgas OLI model incorporates the 5-stage waste feed evaporator model to calculate the composition of the caustic washing total recycle whereas the earlier OLI model did not. The FEP offgas OLI model has the HLW offgas condensate (SBS Recycle) stream whereas the earlier OLI model did not. The earlier model also added a significant amount of acetone to its feed to match the experimental setup whereas the FEP model spiked organic components directly. The earlier model also set the evaporator vapor target at 18450 gmol or 0.29 molar vapor target whereas the FEP model set the vapor target to achieve a certain ultrafiltrate density (nominally at 0.69 molar vapor target). Despite these differences, a comparison of the two task's work would help validate the new FEP offgas OLI model since Hiroshi's work included experimental results. Table XIV shows a side by side comparison of the new FEP offgas regulatory runs described in this document and the earlier OLI model's results. In order to make this comparison, several modifications were made to the FEP OLI model as explained next.

For the first comparison the FEP HLW Offgas Condensate (SBS Recycle) stream and Total Recycle were dropped leaving only the Envelope B/D waste feed into the evaporator. This condition more closely matches the earlier OLI model with only the AN-107 waste feed. Also for this first case, the vapor target of the evaporator in the FEP model was changed from 0.69 to 0.29 to match the earlier OLI model. Note the earlier model was not concerned with the density of the ultrafiltrate stream whereas the FEP model evaporator vapor target was set to produce an ultrafiltrate stream with a 1.22 density. Running the FEP model with these modifications, the condensate had about 2 times more benzene, 4-methyl-2-pentanone, toluene, 1,2-dibromoethane, chlorobenzene, 1,2,3-trichloropropane, 1,2,4-trichlorobenzene, and naphthalene than the earlier model's condensate. The FEP condensate had about the same order of magnitude of Pentachlorophenol, Pyrene, Bis(ethylhexyl)phthalate (BEHP), and Aldrin. These results are not surprising since the earlier model had a different feed than the FEP OLI model. Table XV shows the

\footnotetext{
${ }^{11}$ Saito, Hiroshi H., T. Bond Calloway, Daro M. Ferrara, Alexander S. Choi, "Regulatory Off-gas Analysis form the Evaporation of Hanford Simulated Waste Spiked with Organic Compounds," Journal of Air and Waste Management Association, July 2002.
} 
state of the earlier OLI model and FEP OLI model waste feeds. Table XVI shows a comparison of the composition of the different waste feeds as well as the HLW offgas condensate (SBS Recycle) and total recycle feeds. The FEP waste feed has $25 \%$ more water by mass than the earlier model's waste feed. To evaporate this extra water more energy is put into the evaporator and thus more of the volatile and semivolatile organics are carried up with the evaporator overhead into the condenser where they then exit via the condensate.

As a second comparison, the FEP OLI model was run with the evaporator vapor target of 0.69 which gives the desired ultrafiltrate density when the HLW offgas condensate (SBS Recycle) and total recycle streams are present. For this run these two streams were not included to examine the impact of the evaporator vapor target. By essentially doubling the amount of vapor going out the evaporator, the amount of most volatile organics in the condensate doubled from the prior run. In a few cases, the amounts of Bis(ethylhexyl)phthalate, Dibenz[a,h]anthracene, and Diethyl phthalate in the condensate tripled. However, the amounts of phenol, Hexachlorobenzene, Pyrene, Benzo(a)pyrene, and gamma-BHC (Lindane) stayed about the same. These species have high boiling points and are less affected by the additional heat coming into the system.

For a third comparison, the HLW Offgas Condensate (SBS Recycle) and Total recycle streams were added back to the FEP OLI model in the same proportion as the current FEP model and the evaporator vapor target was kept at 0.69 to achieve 1.22 density for the ultrafiltrate stream. This run does not match the proper production rate but has all feed streams in the right proportion. In this FEP run the amounts of most organics in the condensate again doubled from the prior run. In some cases, (e.g. phenol) the amount tripled in the condensate. These increases are due to the large amount of water added via the HLW offgas condensate and total recycle streams. To evaporate this extra water required about twice the heat duty. As a result, the volatile and semi-volatile species going out overhead essentially doubled. For 4-methyl-2pentanone (MIBK) , Hexachlorobenzene , Pentachlorophenol , Pyrene , Benzo(a)pyrene , 2Chloronaphthalene, Diethyl phthalate, Aldrin, and gamma-BHC (Lindane) the amount in the condensate stayed on the same order of magnitude. Again these species have the higher boiling points and are less affected by the increased heat entering the system. 
WSRC-TR-2002-00550, Rev. 1 SRT-RPP-2002-0027

Table XII. Organic and Mercury Partitioning of Modeling Ultrafiltrate 1.22 Density Case $^{8}$

\begin{tabular}{|c|c|c|c|c|c|c|c|c|c|c|c|c|c|c|}
\hline \multirow[b]{2}{*}{ Stream } & & & & \multicolumn{11}{|c|}{ \% of Evap Feed+Total recycle } \\
\hline & & $\begin{array}{l}\text { Total } \\
\text { Recycle }\end{array}$ & $\begin{array}{l}\text { Evap } \\
\text { Feed }\end{array}$ & $\begin{array}{l}\text { Over- } \\
\text { head }\end{array}$ & $\begin{array}{l}\text { Cooled } \\
\text { Evap } \\
\text { Bott }\end{array}$ & \begin{tabular}{c|} 
PC \\
Vapor
\end{tabular} & $\begin{array}{c}\mathrm{PC} \\
\text { Conden- } \\
\text { sate }\end{array}$ & IC Vapor & $\begin{array}{c}\text { IC } \\
\text { Conden- } \\
\text { sate }\end{array}$ & \begin{tabular}{c|}
$\mathrm{AC}$ \\
Vapor
\end{tabular} & $\begin{array}{c}\mathrm{AC} \\
\text { Conden- } \\
\text { sate }\end{array}$ & $\begin{array}{l}\text { Demist } \\
\text { Vapor }\end{array}$ & $\begin{array}{l}\text { Demist } \\
\text { Liquid }\end{array}$ & $\begin{array}{c}\text { Evap } \\
\text { Cond Tk } \\
\text { Out }\end{array}$ \\
\hline Phase & & Mixed & Mixed & & & & & & & & & & & \\
\hline Temperature, C & & 25 & 25 & & & & & & & & & & & \\
\hline Pressure, atm & & 1 & 1 & & & & & & & & & & & \\
\hline $\mathrm{pH}$ & & 13.4748 & 13.0705 & & & & & & & & & & & \\
\hline Total $\mathrm{mol} / \mathrm{hr}$ & & 4402.02 & 197463 & & & & & & & & & & & \\
\hline Flow Units & & $\mathrm{g} / \mathrm{hr}$ & $\mathrm{g} / \mathrm{hr}$ & & & & & & & & & & & \\
\hline ALDRINE & Aldrin & $1.57 \mathrm{E}-04$ & 4.195 & $85.66 \%$ & $14.34 \%$ & $0.22 \%$ & $85.44 \%$ & $0.07 \%$ & $0.15 \%$ & $0.01 \%$ & $0.06 \%$ & $0.01 \%$ & $0.00 \%$ & $85.65 \%$ \\
\hline BENZENE & Benzene & $7.74 \mathrm{E}-07$ & 4.194 & $100 \%$ & $0.00 \%$ & $90.23 \%$ & $9.76 \%$ & $90.01 \%$ & $0.22 \%$ & $89.26 \%$ & $0.75 \%$ & $89.26 \%$ & $0.00 \%$ & $10.74 \%$ \\
\hline \begin{tabular}{|l|} 
BETHXPHTH2 \\
\end{tabular} & $\begin{array}{l}\text { Bis(2-ethylhexyl) } \\
\text { phthalate (BEHP) }\end{array}$ & $1.97 \mathrm{E}-03$ & 4.193 & $42.70 \%$ & $57.30 \%$ & $0.01 \%$ & $42.69 \%$ & $0.00 \%$ & $0.01 \%$ & $0.00 \%$ & $0.00 \%$ & $0.00 \%$ & $0.00 \%$ & $42.70 \%$ \\
\hline BNZPYREN & Benzo(a)pyrene (BaP) & $9.24 \mathrm{E}-06$ & 4.181 & $0.00 \%$ & $100 \%$ & $0.00 \%$ & $0.00 \%$ & $0.00 \%$ & $0.00 \%$ & $0.00 \%$ & $0.00 \%$ & $0.00 \%$ & $0.00 \%$ & $0.00 \%$ \\
\hline $\mathrm{C6H5OH}$ & Phenol & $2.75 E-02$ & 4.196 & $0.15 \%$ & $99.85 \%$ & $0.00 \%$ & $0.15 \%$ & $0.00 \%$ & $0.00 \%$ & $0.00 \%$ & $0.00 \%$ & $0.00 \%$ & $0.00 \%$ & $0.15 \%$ \\
\hline CL5PHENOL & Pentachlorophenol & $2.37 \mathrm{E}-03$ & 4.196 & $90.84 \%$ & $9.16 \%$ & $0.14 \%$ & $90.69 \%$ & $0.01 \%$ & $0.14 \%$ & $0.00 \%$ & $0.01 \%$ & $0.00 \%$ & $0.00 \%$ & $90.83 \%$ \\
\hline CL6BENZEN & Hexachlorobenzene & $2.20 \mathrm{E}-05$ & 4.188 & $0.00 \%$ & $100 \%$ & $0.00 \%$ & $0.00 \%$ & $0.00 \%$ & $0.00 \%$ & $0.00 \%$ & $0.00 \%$ & $0.00 \%$ & $0.00 \%$ & $0.00 \%$ \\
\hline CLBENZEN & Chlorobenzene & \begin{tabular}{|c|}
$1.66 \mathrm{E}-06$ \\
\end{tabular} & 4.190 & $99.99 \%$ & $0.01 \%$ & $82.59 \%$ & $17.40 \%$ & $82.19 \%$ & $0.40 \%$ & $80.87 \%$ & $1.32 \%$ & $80.87 \%$ & $0.00 \%$ & $19.13 \%$ \\
\hline CLNAPHTH2 & 2-Chloronaphthalene & $4.46 E-06$ & 4.194 & $99.98 \%$ & $0.02 \%$ & $50.84 \%$ & $49.14 \%$ & $49.76 \%$ & $1.08 \%$ & $46.38 \%$ & $3.39 \%$ & $46.37 \%$ & $0.01 \%$ & $53.61 \%$ \\
\hline DBANTHAH & Dibenz[a,h]anthracene & $1.10 E-03$ & 4.191 & $0.22 \%$ & $99.78 \%$ & $0.00 \%$ & $0.22 \%$ & $0.00 \%$ & $0.00 \%$ & $0.00 \%$ & $0.00 \%$ & $0.00 \%$ & $0.00 \%$ & $0.22 \%$ \\
\hline DBRE12 & 1,2-dibromoethane & $3.99 \mathrm{E}-05$ & 28.063 & $99.98 \%$ & $0.02 \%$ & $53.16 \%$ & $46.82 \%$ & $52.10 \%$ & $1.06 \%$ & $48.76 \%$ & $3.34 \%$ & $48.76 \%$ & $0.00 \%$ & $51.22 \%$ \\
\hline DIETPHTHL & Diethyl phthalate & $5.99 E-03$ & 4.202 & $78.05 \%$ & $21.94 \%$ & $0.04 \%$ & $78.02 \%$ & $0.00 \%$ & $0.04 \%$ & $0.00 \%$ & $0.00 \%$ & $0.00 \%$ & $0.00 \%$ & $78.06 \%$ \\
\hline HGCL2 & Mercury Chloride & & & $4.60 \%$ & $96.40 \%$ & $0.00 \%$ & $0.00 \%$ & $0.00 \%$ & $0.00 \%$ & $0.00 \%$ & $0.00 \%$ & $0.00 \%$ & $0.00 \%$ & $0.00 \%$ \\
\hline HGEL & Mercury (sol) & $3.80 \mathrm{E}-06$ & & $100 \%$ & $0.00 \%$ & $12.26 \%$ & $87.73 \%$ & $5.37 \%$ & $6.90 \%$ & $1.53 \%$ & $3.84 \%$ & $1.22 \%$ & $0.32 \%$ & $98.78 \%$ \\
\hline $\mathrm{HGO}$ & Mercuric Oxide (insol) & $9.85 \mathrm{E}-01$ & 136.067 & $0.00 \%$ & $100 \%$ & $0.00 \%$ & $0.00 \%$ & $0.00 \%$ & $0.00 \%$ & $0.00 \%$ & $0.00 \%$ & $0.00 \%$ & $0.00 \%$ & $0.00 \%$ \\
\hline HXCL13BD & Hexachlorobutadiene & $3.47 E-08$ & 4.200 & $100.00 \%$ & $0.00 \%$ & $99.28 \%$ & $0.72 \%$ & $99.27 \%$ & $0.01 \%$ & $99.23 \%$ & $0.04 \%$ & $99.23 \%$ & $0.00 \%$ & $0.77 \%$ \\
\hline LINDANE & gamma-BHC (Lindane) & $9.69 E-05$ & 4.209 & $99.56 \%$ & $0.44 \%$ & $5.93 \%$ & $93.64 \%$ & $4.43 \%$ & $1.49 \%$ & $2.08 \%$ & $2.35 \%$ & $2.08 \%$ & $0.00 \%$ & $97.49 \%$ \\
\hline M4PNTON2 & $\begin{array}{c}\text { 4-methyl-2-pentanone } \\
\text { (MIBK) }\end{array}$ & $1.85 \mathrm{E}-05$ & 4.197 & $99.93 \%$ & $0.07 \%$ & $23.37 \%$ & $76.56 \%$ & $21.73 \%$ & $1.64 \%$ & $17.33 \%$ & $4.41 \%$ & $17.32 \%$ & $0.00 \%$ & $82.61 \%$ \\
\hline NAPHTHALEN & Naphthalene & $4.91 \mathrm{E}-06$ & 4.188 & $99.98 \%$ & $0.02 \%$ & $60.12 \%$ & $39.86 \%$ & $59.22 \%$ & $0.90 \%$ & $56.35 \%$ & $2.87 \%$ & $56.34 \%$ & $0.00 \%$ & $43.64 \%$ \\
\hline PYRENE & Pyrene & \begin{tabular}{|l}
$1.52 \mathrm{E}-04$ \\
\end{tabular} & 4.202 & $96.12 \%$ & $3.88 \%$ & $1.06 \%$ & $95.06 \%$ & $0.56 \%$ & $0.50 \%$ & $0.14 \%$ & $0.43 \%$ & $0.13 \%$ & $0.01 \%$ & $96.00 \%$ \\
\hline TCLBNZ124 & 1,2,4-trichlorobenzene & $1.04 \mathrm{E}-06$ & 4.193 & $100.00 \%$ & $0.00 \%$ & $83.04 \%$ & $16.96 \%$ & $82.66 \%$ & $0.38 \%$ & $81.40 \%$ & $1.26 \%$ & $81.40 \%$ & $0.00 \%$ & $18.60 \%$ \\
\hline TCLPRP123 & 1,2,3-trichloropropane & 7.30E-06 & 4.198 & $99.97 \%$ & $0.03 \%$ & $44.56 \%$ & $55.42 \%$ & $43.32 \%$ & $1.24 \%$ & $39.51 \%$ & $3.81 \%$ & $39.51 \%$ & $0.00 \%$ & $60.47 \%$ \\
\hline TOLUENE & Toluene & \begin{tabular}{|l|}
$6.43 \mathrm{E}-07$ \\
\end{tabular} & 4.194 & $100.00 \%$ & $0.00 \%$ & $91.37 \%$ & $8.63 \%$ & $91.17 \%$ & $0.20 \%$ & $90.50 \%$ & $0.66 \%$ & $90.50 \%$ & $0.00 \%$ & $9.49 \%$ \\
\hline Total $\mathrm{g} / \mathrm{hr}$ & & \begin{tabular}{|l|}
85260.4 \\
\end{tabular} & $4.01 \mathrm{E}+06$ & & & & & & & & & & & \\
\hline Volume, L/hr & & 79.456 & 3550.76 & & & & & & & & & & & \\
\hline
\end{tabular}


WSRC-TR-2002-00550, Rev. 1 SRT-RPP-2002-0027

Table XIII. Organic and Mercury Partitioning of Modeling Ultrafiltrate 1.35 Density Case $^{8}$

\begin{tabular}{|c|c|c|c|c|c|c|c|c|c|c|c|c|c|c|}
\hline & & & & \multicolumn{11}{|c|}{$\%$ of Evap Feed+Total recycle } \\
\hline Stream & & $\begin{array}{c}\text { Total } \\
\text { Recycle }\end{array}$ & $\begin{array}{l}\text { Evap } \\
\text { Feed }\end{array}$ & $\begin{array}{l}\text { Over- } \\
\text { head }\end{array}$ & $\begin{array}{c}\text { Cooled } \\
\text { Evap } \\
\text { Bott }\end{array}$ & PC Vapor & $\begin{array}{c}\mathrm{PC} \\
\text { Conden- } \\
\text { sate }\end{array}$ & IC Vapor & $\begin{array}{c}\mathrm{IC} \\
\text { Condensa } \\
\text { te }\end{array}$ & $\begin{array}{c}\text { AC } \\
\text { Vapor }\end{array}$ & $\begin{array}{c}\mathrm{AC} \\
\text { Conden- } \\
\text { sate }\end{array}$ & $\begin{array}{l}\text { Demist } \\
\text { Vapor }\end{array}$ & $\begin{array}{l}\text { Demist } \\
\text { Liquid }\end{array}$ & $\begin{array}{c}\text { Evap } \\
\text { Cond Tk } \\
\text { Out }\end{array}$ \\
\hline Phase & & Mixed & Mixed & & & & & & & & & & & \\
\hline Temperature, $\mathrm{C}$ & & 25 & 25 & & & & & & & & & & & \\
\hline Pressure, atm & & 1 & 1 & & & & & & & & & & & \\
\hline $\mathrm{pH}$ & & 12.8607 & 13.0705 & & & & & & & & & & & \\
\hline Total $\mathrm{mol} / \mathrm{hr}$ & & 7912.79 & 197463 & & & & & & & & & & & \\
\hline Flow Units & & $\mathrm{g} / \mathrm{hr}$ & $\mathrm{g} / \mathrm{hr}$ & & & & & & & & & & & \\
\hline ALDRINE & Aldrin & $7.20 \mathrm{E}-05$ & 4.195 & $89.58 \%$ & $10.42 \%$ & $0.22 \%$ & $89.36 \%$ & $0.08 \%$ & $0.14 \%$ & $0.01 \%$ & $0.07 \%$ & $0.01 \%$ & $0.00 \%$ & $89.57 \%$ \\
\hline BENZENE & Benzene & $3.54 \mathrm{E}-07$ & 4.194 & $100.00 \%$ & $0.00 \%$ & $88.52 \%$ & $11.47 \%$ & $88.30 \%$ & $0.22 \%$ & $87.57 \%$ & $0.74 \%$ & $87.57 \%$ & $0.00 \%$ & $12.43 \%$ \\
\hline BETHXРНTH2 & $\begin{array}{l}\text { Bis(2-ethylhexyl) } \\
\text { phthalate (BEHP) }\end{array}$ & $9.54 \mathrm{E}-04$ & 4.193 & $53.47 \%$ & $46.53 \%$ & $0.01 \%$ & $53.46 \%$ & $0.00 \%$ & $0.01 \%$ & $0.00 \%$ & $0.00 \%$ & $0.00 \%$ & $0.00 \%$ & $53.47 \%$ \\
\hline BNZPYREN & Benzo(a)pyrene (BaP) & $6.75 \mathrm{E}-06$ & 4.181 & $0.00 \%$ & $100.00 \%$ & $0.00 \%$ & $0.00 \%$ & $0.00 \%$ & $0.00 \%$ & $0.00 \%$ & $0.00 \%$ & $0.00 \%$ & $0.00 \%$ & $0.00 \%$ \\
\hline $\mathrm{C6H5OH}$ & Phenol & $5.36 E-02$ & 4.196 & $0.11 \%$ & $99.89 \%$ & $0.00 \%$ & $0.11 \%$ & $0.00 \%$ & $0.00 \%$ & $0.00 \%$ & $0.00 \%$ & $0.00 \%$ & $0.00 \%$ & $0.11 \%$ \\
\hline CL5PHENOL & Pentachlorophenol & $1.16 \mathrm{E}-03$ & 4.196 & $97.37 \%$ & $2.62 \%$ & $0.13 \%$ & $97.24 \%$ & $0.01 \%$ & $0.12 \%$ & $0.00 \%$ & $0.01 \%$ & $0.00 \%$ & $0.00 \%$ & $97.37 \%$ \\
\hline CL6BENZEN & Hexachlorobenzene & $1.61 \mathrm{E}-05$ & 4.188 & $0.00 \%$ & $100.00 \%$ & $0.00 \%$ & $0.00 \%$ & $0.00 \%$ & $0.00 \%$ & $0.00 \%$ & $0.00 \%$ & $0.00 \%$ & $0.00 \%$ & $0.00 \%$ \\
\hline CLBENZEN & Chlorobenzene & $7.59 \mathrm{E}-07$ & 4.190 & $100.00 \%$ & $0.00 \%$ & $79.84 \%$ & $20.16 \%$ & $79.46 \%$ & $0.38 \%$ & $78.18 \%$ & $1.28 \%$ & $78.18 \%$ & $0.00 \%$ & $21.82 \%$ \\
\hline CLNAPHTH2 & 2-Chloronaphthalene & $2.04 E-06$ & 4.194 & $99.99 \%$ & $0.01 \%$ & $46.50 \%$ & $53.49 \%$ & $45.52 \%$ & $0.98 \%$ & $42.43 \%$ & $3.09 \%$ & $42.42 \%$ & $0.00 \%$ & $57.57 \%$ \\
\hline DBANTHAH & Dibenz[a,h]anthracene & $5.20 E-04$ & 4.191 & $0.30 \%$ & $99.70 \%$ & $0.00 \%$ & $0.30 \%$ & $0.00 \%$ & $0.00 \%$ & $0.00 \%$ & $0.00 \%$ & $0.00 \%$ & $0.00 \%$ & $0.30 \%$ \\
\hline DBRE12 & 1,2-dibromoethane & $1.82 \mathrm{E}-05$ & 28.063 & $100.00 \%$ & $0.01 \%$ & $48.65 \%$ & $51.34 \%$ & $47.69 \%$ & $0.97 \%$ & $44.63 \%$ & $3.06 \%$ & $44.63 \%$ & $0.00 \%$ & $55.36 \%$ \\
\hline DIETPHTHL & Diethyl phthalate & $3.29 E-03$ & 4.202 & $93.72 \%$ & $6.28 \%$ & $0.04 \%$ & $93.69 \%$ & $0.00 \%$ & $0.04 \%$ & $0.00 \%$ & $0.00 \%$ & $0.00 \%$ & $0.00 \%$ & $93.72 \%$ \\
\hline HGCL2 & Mercury Chloride & & & $4.60 \%$ & $96.40 \%$ & $0.00 \%$ & $0.00 \%$ & $0.00 \%$ & $0.00 \%$ & $0.00 \%$ & $0.00 \%$ & $0.00 \%$ & $0.00 \%$ & $0.00 \%$ \\
\hline HGEL & Mercury (sol) & $1.74 \mathrm{E}-06$ & & $100.00 \%$ & $0.00 \%$ & $12.21 \%$ & $87.79 \%$ & $5.35 \%$ & $6.87 \%$ & $1.53 \%$ & $3.82 \%$ & $1.21 \%$ & $0.32 \%$ & $98.79 \%$ \\
\hline $\mathrm{HGO}$ & Mercuric Oxide (insol) & $1.53 \mathrm{E}+00$ & 136.067 & $0.00 \%$ & $100.00 \%$ & $0.00 \%$ & $0.00 \%$ & $0.00 \%$ & $0.00 \%$ & $0.00 \%$ & $0.00 \%$ & $0.00 \%$ & $0.00 \%$ & $0.00 \%$ \\
\hline HXCL13BD & Hexachlorobutadiene & $1.59 E-08$ & 4.200 & $100.00 \%$ & $0.00 \%$ & $99.17 \%$ & $0.83 \%$ & $99.16 \%$ & $0.01 \%$ & $99.12 \%$ & $0.04 \%$ & $99.12 \%$ & $0.00 \%$ & $0.88 \%$ \\
\hline LINDANE & gamma-BHC (Lindane $)$ & $4.44 E-05$ & 4.209 & $99.85 \%$ & $0.15 \%$ & $5.08 \%$ & $94.77 \%$ & $3.81 \%$ & $1.28 \%$ & $1.79 \%$ & $2.02 \%$ & $1.78 \%$ & $0.00 \%$ & $98.07 \%$ \\
\hline M4PNTON2 & $\begin{array}{l}\text { 4-methyl-2-pentanone } \\
\text { (MIBK) }\end{array}$ & $8.46 \mathrm{E}-06$ & 4.197 & $99.98 \%$ & $0.02 \%$ & $20.30 \%$ & $79.68 \%$ & $18.88 \%$ & $1.42 \%$ & $15.05 \%$ & $3.83 \%$ & $15.05 \%$ & $0.00 \%$ & $84.94 \%$ \\
\hline NAPHTHALEN & Naphthalene & $2.24 \mathrm{E}-06$ & 4.188 & $100.00 \%$ & $0.00 \%$ & $55.77 \%$ & $44.23 \%$ & $54.94 \%$ & $0.83 \%$ & $52.27 \%$ & $2.67 \%$ & $52.27 \%$ & $0.00 \%$ & $47.73 \%$ \\
\hline PYRENE & Pyrene & $7.00 \mathrm{E}-05$ & 4.202 & $97.35 \%$ & $2.65 \%$ & $1.03 \%$ & $96.31 \%$ & $0.56 \%$ & $0.47 \%$ & $0.14 \%$ & $0.42 \%$ & $0.13 \%$ & $0.01 \%$ & $97.21 \%$ \\
\hline TCLBNZ124 & 1,2,4-trichlorobenzene & $4.73 \mathrm{E}-07$ & 4.193 & $100.00 \%$ & $0.00 \%$ & $80.43 \%$ & $19.57 \%$ & $80.06 \%$ & $0.37 \%$ & $78.85 \%$ & $1.22 \%$ & $78.84 \%$ & $0.00 \%$ & $21.16 \%$ \\
\hline TCLPRP123 & 1,2,3-trichloropropane & $3.34 \mathrm{E}-06$ & 4.198 & $99.99 \%$ & $0.01 \%$ & $40.16 \%$ & $59.83 \%$ & $39.05 \%$ & $1.12 \%$ & $35.61 \%$ & $3.43 \%$ & $35.61 \%$ & $0.00 \%$ & $64.39 \%$ \\
\hline TOLUENE & Toluene & \begin{tabular}{|l|}
$2.94 \mathrm{E}-07$ \\
\end{tabular} & 4.194 & $100.00 \%$ & $0.00 \%$ & $89.83 \%$ & $10.17 \%$ & $89.64 \%$ & $0.19 \%$ & $88.99 \%$ & $0.65 \%$ & $88.99 \%$ & $0.00 \%$ & $11.01 \%$ \\
\hline Total $\mathrm{g} / \mathrm{hr}$ & & 152823 & $4.01 \mathrm{E}+06$ & & & & & & & & & & & \\
\hline Volume, L/hr & & 143.624 & 3550.76 & & & & & & & & & & & \\
\hline
\end{tabular}


WSRC-TR-2002-00550, Rev. 1 SRT-RPP-2002-0027

Table XIV . Organic Splits compared with Earlier Modeling Work

\begin{tabular}{|c|c|c|c|c|c|c|c|c|c|c|c|c|c|c|c|}
\hline \multirow[b]{2}{*}{$\begin{array}{l}\text { evaporator vapor target (molar } \\
\text { fraction of feed)= }\end{array}$} & \multicolumn{3}{|c|}{ EXPERIMENTAL DATA } & \multicolumn{3}{|c|}{ Original OLI Model } & \multicolumn{3}{|c|}{$\begin{array}{l}\text { FEP Model with Waste Feed } \\
\text { only and evap vapor target like } \\
\text { Original OLI Model }\end{array}$} & \multicolumn{3}{|c|}{$\begin{array}{l}\text { FEP Model with Waste Feed } \\
\text { only and evap vapor target for } \\
1.22 \text { UF density }\end{array}$} & \multicolumn{3}{|c|}{$\begin{array}{l}\text { FEP Model with Waste Feed, } \\
\text { HLW OG Condensate, Total } \\
\text { Recycle and evap vapor target } \\
\text { for } 1.22 \text { UF density }\end{array}$} \\
\hline & & & & 0.29 & & & 0.29 & & & 0.69 & & & 0.69 & & \\
\hline Evaporator Concentrate rho $=$ & & & & 1.38 & & & 1.18 & & & 1.39 & & & 1.22 & & \\
\hline UF1 Filtrate rho= & & & & & & & 1.18 & & & 1.37 & & & 1.22 & & \\
\hline Target Organic & $\begin{array}{l}\text { Conden- } \\
\text { sate }\end{array}$ & $\begin{array}{l}\text { Concen- } \\
\text { trate }\end{array}$ & Off-gas & $\begin{array}{l}\text { Conden- } \\
\text { sate= } \\
\text { REG_C } \\
\text { Condens } \\
\text { ate }\end{array}$ & \begin{tabular}{|l} 
Concen- \\
trate= \\
REG_C \\
Final \\
Concntra \\
te
\end{tabular} & \begin{tabular}{|l|} 
Off-gas \\
=REG_C \\
Vent
\end{tabular} & $\begin{array}{l}\text { Conden- } \\
\text { sate= } \\
\text { FEP PC } \\
\text { Condens } \\
\text { ate }\end{array}$ & $\begin{array}{l}\text { Concen- } \\
\text { trate= } \\
\text { FEP } \\
\text { Cooled } \\
\text { Evap } \\
\text { Bott } \\
\end{array}$ & $\begin{array}{l}\text { Off-gas } \\
=\text { FEP PC } \\
\text { Vapor }\end{array}$ & $\begin{array}{l}\text { Conden- } \\
\text { sate= } \\
\text { FEP PC } \\
\text { Condens } \\
\text { ate }\end{array}$ & $\begin{array}{l}\text { Concen- } \\
\text { trate= } \\
\text { FEP } \\
\text { Cooled } \\
\text { Evap } \\
\text { Bott } \\
\end{array}$ & $\begin{array}{l}\text { Off-gas } \\
=\text { FEP PC } \\
\text { Vapor }\end{array}$ & $\begin{array}{l}\text { Conden- } \\
\text { sate= } \\
\text { FEP PC } \\
\text { Condens } \\
\text { ate }\end{array}$ & $\begin{array}{l}\text { Concen- } \\
\text { trate= } \\
\text { FEP } \\
\text { Cooled } \\
\text { Evap } \\
\text { Bott }\end{array}$ & $\begin{array}{l}\text { Off-gas } \\
=\text { FEP PC } \\
\text { Vapor }\end{array}$ \\
\hline Benzene & $0.7 \%$ & $0.3 \% *$ & $89.8 \%$ & $0.4 \%$ & $0.0 \%$ & $99.6 \%$ & $0.7 \%$ & $0.0 \%$ & $99.2 \%$ & $1.7 \%$ & $0.0 \%$ & $98.3 \%$ & $3.4 \%$ & $0.0 \%$ & $96.6 \%$ \\
\hline 4-methyl-2-pentanone (MIBK) & $2.1 \% *$ & $1.8 \% *$ & $66.8 \%$ & $11.9 \%$ & $0.2 \%$ & $88.0 \%$ & $18.1 \%$ & $0.5 \%$ & $81.4 \%$ & $34.9 \%$ & $0.0 \%$ & $65.0 \%$ & $51.5 \%$ & $0.1 \%$ & $48.4 \%$ \\
\hline Toluene & $0.8 \%$ & $0.6 \% *$ & $55.0 \%$ & $0.4 \%$ & $0.0 \%$ & $99.6 \%$ & $0.6 \%$ & $0.0 \%$ & $99.3 \%$ & $1.5 \%$ & $0.0 \%$ & $98.5 \%$ & $3.0 \%$ & $0.0 \%$ & $97.0 \%$ \\
\hline 1,2-dibromoethane & $18.6 \%$ & $2.0 \% *$ & $331.7 \%$ & $3.5 \%$ & $0.1 \%$ & $96.4 \%$ & $5.6 \%$ & $0.2 \%$ & $94.2 \%$ & $12.6 \%$ & $0.0 \%$ & $87.4 \%$ & $22.3 \%$ & $0.0 \%$ & $77.7 \%$ \\
\hline 1,2,3-trichloropropane & $5.6 \% *$ & $16.2 \% *$ & $0.1 \%$ & $4.9 \%$ & $0.1 \%$ & $95.1 \%$ & $7.8 \%$ & $0.2 \%$ & $92.0 \%$ & $16.9 \%$ & $0.0 \%$ & $83.1 \%$ & $28.8 \%$ & $0.0 \%$ & $71.2 \%$ \\
\hline phenol & & & & & & & $0.0 \%$ & $100.0 \%$ & $0.0 \%$ & $0.0 \%$ & $100.0 \%$ & $0.0 \%$ & $0.2 \%$ & $99.8 \%$ & $0.0 \%$ \\
\hline Hexachlorobutadiene & & & & & & & $0.1 \%$ & $0.0 \%$ & $99.9 \%$ & $0.1 \%$ & $0.0 \%$ & $99.9 \%$ & $0.2 \%$ & $0.0 \%$ & $99.8 \%$ \\
\hline 1,2,4-trichlorobenzene & $4.4 \%$ & $6.6 \% *$ & $86.1 \%$ & $1.0 \%$ & $0.0 \%$ & $99.0 \%$ & $1.5 \%$ & $0.0 \%$ & $98.5 \%$ & $3.3 \%$ & $0.0 \%$ & $96.7 \%$ & $6.2 \%$ & $0.0 \%$ & $93.8 \%$ \\
\hline Naphthalene & $16.9 \%$ & $3.1 \% *$ & $79.3 \%$ & $2.8 \%$ & $0.1 \%$ & $97.2 \%$ & $4.4 \%$ & $0.1 \%$ & $95.4 \%$ & $9.9 \%$ & $0.0 \%$ & $90.1 \%$ & $17.7 \%$ & $0.0 \%$ & $82.3 \%$ \\
\hline Hexachlorobenzene & $0.1 \%$ & $71.3 \% *$ & $3.0 \%$ & $0.0 \%$ & $100.0 \%$ & $0.0 \%$ & $0.0 \%$ & $100.0 \%$ & $0.0 \%$ & $0.0 \%$ & $100.0 \%$ & $0.0 \%$ & $0.0 \%$ & $100.0 \%$ & $0.0 \%$ \\
\hline Pentachlorophenol & $0.0 \%$ & $174 \%$ & $0.0 \%$ & $77.8 \%$ & $19.4 \%$ & $2.8 \%$ & $57.8 \%$ & $40.9 \%$ & $1.3 \%$ & $93.9 \%$ & $5.2 \%$ & $0.9 \%$ & $90.4 \%$ & $9.2 \%$ & $0.4 \%$ \\
\hline Pyrene & $4.7 \%$ & $56.5 \% *$ & $2.1 \%$ & $88.4 \%$ & $6.0 \%$ & $5.6 \%$ & $74.7 \%$ & $21.7 \%$ & $3.6 \%$ & $89.4 \%$ & $7.2 \%$ & $3.5 \%$ & $92.8 \%$ & $3.9 \%$ & $3.3 \%$ \\
\hline $\begin{array}{l}\text { Bis(ethylhexyl)phthalate } \\
\text { (BEHP) }\end{array}$ & $0.1 \%$ & $61.2 \% *$ & $0.0 \%$ & $12.6 \%$ & $87.4 \%$ & $0.0 \%$ & $7.7 \%$ & $92.3 \%$ & $0.0 \%$ & $22.6 \%$ & $77.4 \%$ & $0.0 \%$ & $42.7 \%$ & $57.3 \%$ & $0.0 \%$ \\
\hline Benzo(a)pyrene & $0.0 \%$ & $55.4 \% *$ & $0.0 \%$ & $0.0 \%$ & $100.0 \%$ & $0.0 \%$ & $0.0 \%$ & $100.0 \%$ & $0.0 \%$ & $0.0 \%$ & $100.0 \%$ & $0.0 \%$ & $0.0 \%$ & $100.0 \%$ & $0.0 \%$ \\
\hline 2-Chloronaphthalene & & & & & & & $6.9 \%$ & $0.1 \%$ & $93.0 \%$ & $14.1 \%$ & $0.0 \%$ & $85.9 \%$ & $23.9 \%$ & $0.0 \%$ & $76.1 \%$ \\
\hline Dibenz[a,h]anthracene & & & & & & & $0.0 \%$ & $100.0 \%$ & $0.0 \%$ & $0.1 \%$ & $99.9 \%$ & $0.0 \%$ & $0.2 \%$ & $99.8 \%$ & $0.0 \%$ \\
\hline Diethyl phthalate & & & & & & & $33.8 \%$ & $66.0 \%$ & $0.2 \%$ & $88.3 \%$ & $11.4 \%$ & $0.3 \%$ & $77.9 \%$ & $22.0 \%$ & $0.1 \%$ \\
\hline
\end{tabular}


Table XV. Hiroshi and FEP Model Waste Feeds

\begin{tabular}{|c|c|c|c|c|}
\hline & Hiroshi Feed & \multicolumn{3}{|c|}{ FEP Feeds } \\
\hline Stream & $\begin{array}{c}\text { Waste Feed } \\
\text { (Feed to Evap) }\end{array}$ & $\begin{array}{c}\text { Waste Feed } \\
\text { (EVAP } \\
\text { REDRXN } \\
\text { OUT) }\end{array}$ & $\begin{array}{c}\text { HLW Offgas } \\
\text { Condensate } \\
\text { (HLW OG Cond) }\end{array}$ & Total Recycle \\
\hline Phase & Mixed & Mixed & Mixed & Mixed \\
\hline Temperature, C & 25 & 25 & 25 & 25 \\
\hline Pressure, atm & 1 & 1 & 1 & 1 \\
\hline $\mathrm{pH}$ & 14.0002 & 13.0705 & 11.2694 & 13.4748 \\
\hline Total g/hr & $1.50 \mathrm{E}+06$ & $1.31 \mathrm{E}+06$ & $1.12 \mathrm{E}+06$ & $2.78 \mathrm{E}+04$ \\
\hline
\end{tabular}

Table XVI. Hiroshi and FEP Model Waste Feed Compositions ${ }^{8}$

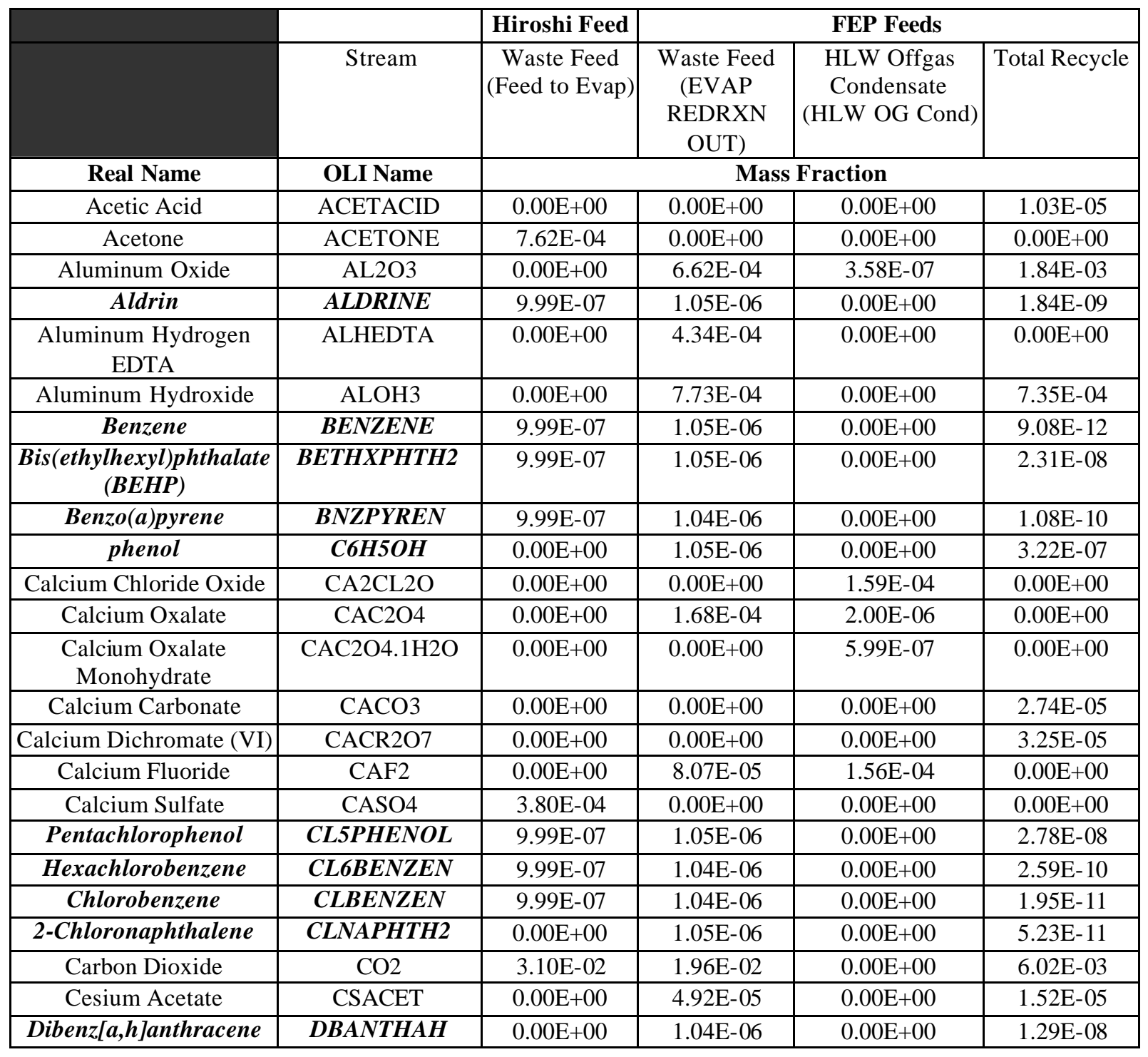


WSRC-TR-2002-00550, Rev. 0 SRT-RPP-2002-00274

\begin{tabular}{|c|c|c|c|c|c|}
\hline & & Hiroshi Feed & \multicolumn{3}{|c|}{ FEP Feeds } \\
\hline & Stream & $\begin{array}{c}\text { Waste Feed } \\
\text { (Feed to Evap) }\end{array}$ & $\begin{array}{c}\text { Waste Feed } \\
\text { (EVAP } \\
\text { REDRXN } \\
\text { OUT) }\end{array}$ & $\begin{array}{c}\text { HLW Offgas } \\
\text { Condensate } \\
\text { (HLW OG Cond) }\end{array}$ & Total Recycle \\
\hline Real Name & OLI Name & & Mas & Fraction & \\
\hline 1,2-dibromoethane & DBRE12 & 9.99E-07 & $6.99 \mathrm{E}-06$ & $0.00 \mathrm{E}+00$ & $4.68 \mathrm{E}-10$ \\
\hline Diethyl Phthalate & DIETPHTHL & $0.00 \mathrm{E}+00$ & $1.05 \mathrm{E}-06$ & $0.00 \mathrm{E}+00$ & $7.03 \mathrm{E}-08$ \\
\hline Iron (III) NTA & FENTA & $1.20 \mathrm{E}-04$ & $0.00 \mathrm{E}+00$ & $0.00 \mathrm{E}+00$ & $0.00 \mathrm{E}+00$ \\
\hline Iron (III) Chloride & FECL3 & $0.00 \mathrm{E}+00$ & $0.00 \mathrm{E}+00$ & $3.37 \mathrm{E}-07$ & $0.00 \mathrm{E}+00$ \\
\hline Iron (III) Oxalate & FEIII2C2O43 & $0.00 \mathrm{E}+00$ & $0.00 \mathrm{E}+00$ & $0.00 \mathrm{E}+00$ & 4.37E-07 \\
\hline $\begin{array}{c}\text { Iron (III) Hydrogen } \\
\text { EDTA }\end{array}$ & FEIIIHEDTA & $0.00 \mathrm{E}+00$ & $0.00 \mathrm{E}+00$ & $0.00 \mathrm{E}+00$ & $1.29 \mathrm{E}-04$ \\
\hline Iron (III) Hydroxide & FEIIIOH3 & $0.00 \mathrm{E}+00$ & $0.00 \mathrm{E}+00$ & $4.50 \mathrm{E}-05$ & $3.42 \mathrm{E}-04$ \\
\hline Gluconic Acid & GLUCONACID & 9.39E-04 & $0.00 \mathrm{E}+00$ & $0.00 \mathrm{E}+00$ & $0.00 \mathrm{E}+00$ \\
\hline 2,2-Iminobisacetic acid & H2IDA & $1.61 \mathrm{E}-03$ & $2.82 \mathrm{E}-04$ & $0.00 \mathrm{E}+00$ & $8.71 \mathrm{E}-05$ \\
\hline Water & $\mathrm{H} 2 \mathrm{O}$ & $6.69 \mathrm{E}-01$ & $8.20 \mathrm{E}-01$ & 9.77E-01 & $8.88 \mathrm{E}-01$ \\
\hline Hydrogen Chloride & $\mathrm{HCL}$ & $0.00 \mathrm{E}+00$ & $0.00 \mathrm{E}+00$ & $5.42 \mathrm{E}-03$ & $9.21 \mathrm{E}-04$ \\
\hline Formic Acid & $\mathrm{HCOOH}$ & $0.00 \mathrm{E}+00$ & $9.87 \mathrm{E}-05$ & $0.00 \mathrm{E}+00$ & $3.05 \mathrm{E}-05$ \\
\hline Hydrogen Floride & $\mathrm{HF}$ & $0.00 \mathrm{E}+00$ & $8.44 \mathrm{E}-04$ & $6.15 \mathrm{E}-06$ & $1.83 \mathrm{E}-04$ \\
\hline Mercury (elemental) & HGEL & $0.00 \mathrm{E}+00$ & $1.57 \mathrm{E}-05$ & $0.00 \mathrm{E}+00$ & $4.46 \mathrm{E}-11$ \\
\hline Mercuric Oxide & $\mathrm{HGO}$ & $0.00 \mathrm{E}+00$ & $1.70 \mathrm{E}-05$ & $0.00 \mathrm{E}+00$ & $1.16 \mathrm{E}-05$ \\
\hline Nitrous(III) Acid & HNO2 & $0.00 \mathrm{E}+00$ & 2.33E-02 & $1.08 \mathrm{E}-03$ & $1.10 \mathrm{E}-02$ \\
\hline Nitric Acid & HNO3 & $0.00 \mathrm{E}+00$ & $1.12 \mathrm{E}-02$ & $5.49 \mathrm{E}-04$ & $2.72 \mathrm{E}-02$ \\
\hline Hexachlorobutadiene & $H X C L 13 B D$ & $0.00 \mathrm{E}+00$ & $1.05 \mathrm{E}-06$ & $0.00 \mathrm{E}+00$ & $4.07 \mathrm{E}-13$ \\
\hline Potassium Citrate & K3CTRT & $0.00 \mathrm{E}+00$ & 7.04E-04 & $0.00 \mathrm{E}+00$ & $2.17 \mathrm{E}-04$ \\
\hline Potassium Formate & $\mathrm{KCOOH}$ & $1.89 \mathrm{E}-03$ & $0.00 \mathrm{E}+00$ & $0.00 \mathrm{E}+00$ & $0.00 \mathrm{E}+00$ \\
\hline Potassium Chloride & $\mathrm{KCL}$ & $0.00 \mathrm{E}+00$ & $3.37 \mathrm{E}-04$ & $5.37 \mathrm{E}-03$ & $2.61 \mathrm{E}-03$ \\
\hline Potassium Glycolate & KGLYCOLAT & $0.00 \mathrm{E}+00$ & $0.00 \mathrm{E}+00$ & $0.00 \mathrm{E}+00$ & $4.08 \mathrm{E}-04$ \\
\hline Potassium Nitrate & KNO3 & $0.00 \mathrm{E}+00$ & $5.93 \mathrm{E}-03$ & $0.00 \mathrm{E}+00$ & $0.00 \mathrm{E}+00$ \\
\hline gamma-BHC (Lindane $)$ & LINDANE & $0.00 \mathrm{E}+00$ & $1.05 \mathrm{E}-06$ & $0.00 \mathrm{E}+00$ & $1.14 \mathrm{E}-09$ \\
\hline $\begin{array}{c}\text { 4-methyl-2-pentanone } \\
(M I B K)\end{array}$ & M4PNTON2 & 9.99E-07 & $1.05 \mathrm{E}-06$ & $0.00 \mathrm{E}+00$ & $2.17 \mathrm{E}-10$ \\
\hline Magnesium Oxalate & MGC2O4 & $0.00 \mathrm{E}+00$ & $0.00 \mathrm{E}+00$ & 5.99E-07 & $0.00 \mathrm{E}+00$ \\
\hline $\begin{array}{c}\text { Magnesium Dichromate } \\
(\mathrm{VI})\end{array}$ & MGCR2O7 & $0.00 \mathrm{E}+00$ & $0.00 \mathrm{E}+00$ & $0.00 \mathrm{E}+00$ & $1.89 \mathrm{E}-09$ \\
\hline Magnesium Hydroxide & MGOH2 & $0.00 \mathrm{E}+00$ & $0.00 \mathrm{E}+00$ & $6.89 \mathrm{E}-05$ & $7.56 \mathrm{E}-06$ \\
\hline Manganese(II) Oxalate & $\mathrm{MNC} 2 \mathrm{O} 4$ & $0.00 \mathrm{E}+00$ & $0.00 \mathrm{E}+00$ & $0.00 \mathrm{E}+00$ & $1.39 \mathrm{E}-06$ \\
\hline Manganese(II) Hydroxide & $\mathrm{MNOH} 2$ & $0.00 \mathrm{E}+00$ & $0.00 \mathrm{E}+00$ & $0.00 \mathrm{E}+00$ & $8.76 \mathrm{E}-06$ \\
\hline Nitrogen & $\mathrm{N} 2$ & $0.00 \mathrm{E}+00$ & $0.00 \mathrm{E}+00$ & $0.00 \mathrm{E}+00$ & $4.47 \mathrm{E}-11$ \\
\hline Sodium Tetraborate & NA2B4O7 & $8.02 \mathrm{E}-05$ & $0.00 \mathrm{E}+00$ & $0.00 \mathrm{E}+00$ & $0.00 \mathrm{E}+00$ \\
\hline Sodium Oxalate & $\mathrm{NA} 2 \mathrm{C} 2 \mathrm{O} 4$ & $1.53 \mathrm{E}-03$ & $3.57 \mathrm{E}-03$ & $0.00 \mathrm{E}+00$ & $2.47 \mathrm{E}-05$ \\
\hline Sodium Chromate (VI) & NA2CRO4 & $1.29 \mathrm{E}-06$ & $2.08 \mathrm{E}-03$ & $0.00 \mathrm{E}+00$ & $6.01 \mathrm{E}-04$ \\
\hline $\begin{array}{c}\text { Disodium Dihydrogen } \\
\text { EDTA } \\
\end{array}$ & NA2H2EDTA & $2.63 \mathrm{E}-03$ & $0.00 \mathrm{E}+00$ & $0.00 \mathrm{E}+00$ & $0.00 \mathrm{E}+00$ \\
\hline Sodium Molybdate (VI) & NA2MOO4 & $3.36 \mathrm{E}-05$ & $0.00 \mathrm{E}+00$ & $0.00 \mathrm{E}+00$ & $0.00 \mathrm{E}+00$ \\
\hline Sodium Metasilicate & NA2SIO3 & $3.13 \mathrm{E}-05$ & $0.00 \mathrm{E}+00$ & $0.00 \mathrm{E}+00$ & $0.00 \mathrm{E}+00$ \\
\hline Sodium Sulfate & NA2SO4 & $5.45 \mathrm{E}-03$ & $0.00 \mathrm{E}+00$ & $0.00 \mathrm{E}+00$ & $0.00 \mathrm{E}+00$ \\
\hline Sodium Orthophosphate & NA3PO4 & $1.24 \mathrm{E}-03$ & $0.00 \mathrm{E}+00$ & $0.00 \mathrm{E}+00$ & $0.00 \mathrm{E}+00$ \\
\hline Sodium Acetate & NAACET & $3.80 \mathrm{E}-04$ & $4.57 \mathrm{E}-05$ & $0.00 \mathrm{E}+00$ & $0.00 \mathrm{E}+00$ \\
\hline
\end{tabular}


WSRC-TR-2002-00550, Rev. 0 SRT-RPP-2002-00274

\begin{tabular}{|c|c|c|c|c|c|}
\hline & & \begin{tabular}{|l|} 
Hiroshi Feed \\
\end{tabular} & \multicolumn{3}{|c|}{ FEP Feeds } \\
\hline & Stream & $\begin{array}{c}\text { Waste Feed } \\
\text { (Feed to Evap) }\end{array}$ & $\begin{array}{l}\text { Waste Feed } \\
\text { (EVAP } \\
\text { REDRXN } \\
\text { OUT) }\end{array}$ & $\begin{array}{c}\text { HLW Offgas } \\
\text { Condensate } \\
\text { (HLW OG Cond) }\end{array}$ & Total Recycle \\
\hline Real Name & OLI Name & \multicolumn{4}{|c|}{ Mass Fraction } \\
\hline Sodium Aluminate & NAALO2 & $5.31 \mathrm{E}-04$ & $0.00 \mathrm{E}+00$ & $0.00 \mathrm{E}+00$ & $0.00 \mathrm{E}+00$ \\
\hline Sodium Chloride & NACL & $1.48 \mathrm{E}-03$ & $0.00 \mathrm{E}+00$ & $0.00 \mathrm{E}+00$ & $0.00 \mathrm{E}+00$ \\
\hline Sodium Formate & $\mathrm{NACOOH}$ & $6.33 \mathrm{E}-03$ & $0.00 \mathrm{E}+00$ & $0.00 \mathrm{E}+00$ & $0.00 \mathrm{E}+00$ \\
\hline Sodium Floride & NAF & $3.51 \mathrm{E}-04$ & $0.00 \mathrm{E}+00$ & $0.00 \mathrm{E}+00$ & $0.00 \mathrm{E}+00$ \\
\hline Sodium Glycolate & NAGLYCOLAT & $6.46 \mathrm{E}-03$ & $1.14 \mathrm{E}-03$ & $0.00 \mathrm{E}+00$ & $0.00 \mathrm{E}+00$ \\
\hline $\begin{array}{c}\text { Sodium Dihydrogen } \\
\text { Citrate }\end{array}$ & NAH2CTRT & $2.80 \mathrm{E}-03$ & $0.00 \mathrm{E}+00$ & $0.00 \mathrm{E}+00$ & $0.00 \mathrm{E}+00$ \\
\hline Sodium Dihydrogen NTA & NAH2NTA & $6.45 \mathrm{E}-05$ & $0.00 \mathrm{E}+00$ & $0.00 \mathrm{E}+00$ & $0.00 \mathrm{E}+00$ \\
\hline Sodium Nitrite & NANO2 & $4.25 \mathrm{E}-02$ & $1.58 \mathrm{E}-02$ & $8.88 \mathrm{E}-04$ & $0.00 \mathrm{E}+00$ \\
\hline Sodium Nitrite & NANO3 & $1.48 \mathrm{E}-01$ & $0.00 \mathrm{E}+00$ & $0.00 \mathrm{E}+00$ & $0.00 \mathrm{E}+00$ \\
\hline Sodium Hydroxide & $\mathrm{NAOH}$ & $7.43 \mathrm{E}-02$ & $8.11 \mathrm{E}-02$ & $7.48 \mathrm{E}-03$ & $5.62 \mathrm{E}-02$ \\
\hline Naphthalene & NAPHTHALEN & 9.99E-07 & $1.04 \mathrm{E}-06$ & $0.00 \mathrm{E}+00$ & $5.75 \mathrm{E}-11$ \\
\hline $\begin{array}{c}\text { Sodium Aluminosilicate } \\
\text { gel }\end{array}$ & NASGEL.15.5H2O & $0.00 \mathrm{E}+00$ & $0.00 \mathrm{E}+00$ & $8.49 \mathrm{E}-04$ & $9.50 \mathrm{E}-04$ \\
\hline Nickel (II) Oxalate & $\mathrm{NIC} 2 \mathrm{O} 4$ & $0.00 \mathrm{E}+00$ & $0.00 \mathrm{E}+00$ & $0.00 \mathrm{E}+00$ & $4.66 \mathrm{E}-05$ \\
\hline Nickel (II) Hydroxide & $\mathrm{NIOH} 2$ & $2.44 \mathrm{E}-04$ & $0.00 \mathrm{E}+00$ & $0.00 \mathrm{E}+00$ & $0.00 \mathrm{E}+00$ \\
\hline Nickel (II) Sulfate & NISO4 & $1.76 \mathrm{E}-04$ & $0.00 \mathrm{E}+00$ & $0.00 \mathrm{E}+00$ & $0.00 \mathrm{E}+00$ \\
\hline Oxygen & $\mathrm{O} 2$ & $0.00 \mathrm{E}+00$ & $0.00 \mathrm{E}+00$ & $0.00 \mathrm{E}+00$ & $2.49 \mathrm{E}-11$ \\
\hline Phosphorus Pentoxide & $\mathrm{P} 2 \mathrm{O} 5$ & $0.00 \mathrm{E}+00$ & $1.59 \mathrm{E}-04$ & $0.00 \mathrm{E}+00$ & $4.92 \mathrm{E}-05$ \\
\hline Pyrene & PYRENE & 9.99E-07 & $1.05 \mathrm{E}-06$ & $0.00 \mathrm{E}+00$ & 1.79E-09 \\
\hline Silicon Dioxide & $\mathrm{SIO} 2$ & $0.00 \mathrm{E}+00$ & $0.00 \mathrm{E}+00$ & $9.71 \mathrm{E}-05$ & $4.18 \mathrm{E}-05$ \\
\hline Sulfur Trioxide & $\mathrm{SO} 3$ & $0.00 \mathrm{E}+00$ & $0.00 \mathrm{E}+00$ & $1.68 \mathrm{E}-04$ & $2.37 \mathrm{E}-03$ \\
\hline Strontium Oxalate & SRC2O4 & $3.67 \mathrm{E}-05$ & $1.17 \mathrm{E}-02$ & $0.00 \mathrm{E}+00$ & $0.00 \mathrm{E}+00$ \\
\hline Strontium Carbonate & SRCO3 & $6.55 \mathrm{E}-05$ & $0.00 \mathrm{E}+00$ & $0.00 \mathrm{E}+00$ & $0.00 \mathrm{E}+00$ \\
\hline 1,2,4-trichlorobenzene & TCLBNZ124 & 9.99E-07 & $1.05 \mathrm{E}-06$ & $0.00 \mathrm{E}+00$ & $1.21 \mathrm{E}-11$ \\
\hline 1,2,3-trichloropropane & TCLPRP123 & 9.99E-07 & $1.05 \mathrm{E}-06$ & $0.00 \mathrm{E}+00$ & $8.56 \mathrm{E}-11$ \\
\hline Toluene & TOLUENE & 9.99E-07 & $1.05 \mathrm{E}-06$ & $0.00 \mathrm{E}+00$ & $7.54 \mathrm{E}-12$ \\
\hline $\begin{array}{l}\text { Zinc Dichloride } \\
\text { Pentoxide }\end{array}$ & ZN6CL2O5 & $0.00 \mathrm{E}+00$ & $0.00 \mathrm{E}+00$ & $2.68 \mathrm{E}-06$ & $0.00 \mathrm{E}+00$ \\
\hline Zinc Oxalate & $\mathrm{ZNC} 2 \mathrm{O} 4$ & $0.00 \mathrm{E}+00$ & $0.00 \mathrm{E}+00$ & $0.00 \mathrm{E}+00$ & $8.76 \mathrm{E}-05$ \\
\hline Zinc Hydroxide & $\mathrm{ZNOH} 2$ & $0.00 \mathrm{E}+00$ & $0.00 \mathrm{E}+00$ & $2.12 \mathrm{E}-04$ & $0.00 \mathrm{E}+00$ \\
\hline Zirconium EDTA & ZREDTA & $0.00 \mathrm{E}+00$ & $0.00 \mathrm{E}+00$ & $0.00 \mathrm{E}+00$ & $1.92 \mathrm{E}-05$ \\
\hline Zirconium Oxide & $\mathrm{ZRO} 2$ & $0.00 \mathrm{E}+00$ & $0.00 \mathrm{E}+00$ & $0.00 \mathrm{E}+00$ & $5.19 \mathrm{E}-05$ \\
\hline & Total & $1.00 \mathrm{E}+00$ & $1.00 \mathrm{E}+00$ & $1.00 \mathrm{E}+00$ & $1.00 \mathrm{E}+00$ \\
\hline
\end{tabular}




\subsection{CONCLUSIONS}

More of the volatile and semi-volatile organics in the evaporator feed go out the primary condenser condensate than in earlier OLI models. These increased rates are due to the larger vapor target of the evaporator and the larger amount of water coming into the evaporator. The greater heat duty for the evaporator causes more of the volatiles and semi-volatiles to go out the overhead and condense out. From these earlier comparisons, adjusting the evaporator target does have a significant impact on the organic partitioning. The amount of water entering the evaporator from the HLW offgas condensate (SBS Recycle) and the total recycle have a significant impact.

This work does not reflect the true conditions of mercury in the waste feed evaporator. For this reason it is strongly suggested that the mercury calculations not be used in the evaporator engineering calculations for design. SRTC will be conducting experiments to attempt to provide a mercury mass balance across the waste feed evaporator. ${ }^{6}$ This new work will be better suited for mercury analysis and engineering design calculations.

The mercury partitioning based on the assumptions made for this task is fairly straightforward based on the OLI modeling. All the elemental mercury in the feed goes out the evaporator overhead. About $5 \%$ of the mercuric chloride in the feed goes out the evaporator overhead. No other mercury species were observed going out the evaporator overhead. All the insoluble mercury goes out the evaporator bottoms. In these OLI models the insoluble mercury was represented as mercuric oxide. How much mercury goes out the evaporator overhead depends on the amount of elemental mercury in the waste feed. For these runs, the soluble mercury in the waste feed came from an assumed amount of soluble mercury based on high contract limits versus actual lower tank values and an assumed $50 \%$ reduction of insoluble mercury to elemental mercury. These assumptions can be changed to better reflect actual plant conditions or updated sample data. The organic partitioning is basically unaffected by the mercury assumptions. Therefore, the results can be applied to cases where there is or is not mercury redox, there is or is not elemental mercury in the feed, and there is or is no insoluble mercury in the feed. For example, if no insoluble mercury is reduced and there is no elemental mercury in the feed then the OLI model predicts that only 5\% of the mercuric chloride in the feed goes out the overhead. The organic partitioning remains the same despite the partitioning of the mercury.

\subsection{ISSUES \& RECOMMENDATIONS}

In gathering data for the two offgas runs, several runs were done to investigate mercury redox. Based on these runs it appears that the built-in redox for mercury must be modified to more closely match observed mercury redox behavior. Some redox behavior can be inserted into the OLI chemistry model by using kinetic equations. However, the reduction and oxidation are then forced to fit a desired result rather than following standard redox chemistry. The mercury redox behavior in OLI should be studied further so it can be refined to more closely match experimental data. Further, the behavior of soluble and insoluble forms of mercury in OLI need to be investigated to address concerns over the partitioning of mercury between the evaporator overhead and bottoms.

In collecting data for the two offgas runs, several runs were made with different amounts of water coming into the offgas train from the feed streams as well as the steam ejectors and recycle streams. The organic partitioning is significantly impacted by the amount of water being fed into the system due to the volatility of the organics and their solubility in water. The amount of water can be controlled by the amount of excess steam going to the steam ejectors as well as the amount of HLW offgas condensate (SBS Recycle) and Ultrafiltration caustic wash that are recycled back to the evaporator. More studies are needed to quantity the impacts of these variables. 


\subsection{APPENDIX A -WASTE FEED EVAPORATOR OFFGAS MODEL RESULTS}

Figure A1 shows the organic partitioning in the waste feed evaporator offgas model for the run with the ultrafiltration filtrate (UF1 Filtrate) at a density of $1.22 \mathrm{~g} / \mathrm{ml}$. Figure A-2 shows organic partitioning in the waste feed evaporator offgas OLI model for the run with ultrafiltration filtrate (UF1 Filtrate) with a density of $1.35 \mathrm{~g} / \mathrm{ml}$. These schematics are provided as one-page summaries of the offgas regulatory run results.

Table A- 1 shows the complete OLI model output for all species for ultrafiltration filtrate (UF1 Filtrate) at a density of $1.22 \mathrm{~g} / \mathrm{ml}$. Table A- 2 shows the complete model output for all species for ultrafiltration filtrate (UF1 Filtrate) at a density of $1.35 \mathrm{~g} / \mathrm{ml}$. From this data all prior discussed results are derived. 


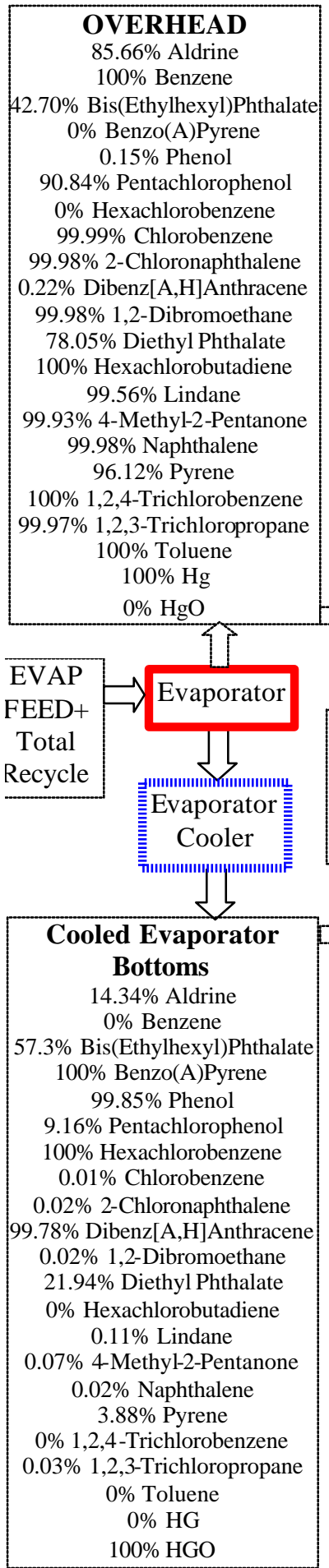

\section{PC Vapor
$0.22 \%$ Aldrine \\ 90.23\% Benzene}

$0.01 \%$ Bis(Ethylhexyl)Phthalat

$0 \%$ Benzo(A)Pyrene 0\% Phenol

$0.14 \%$ Pentachlorophenol

$0 \%$ Hexachlorobenzene

82.59\% Chlorobenzene

50.84\%2-Chloronaphthalene

0\% Dibenz[A,H]Anthracene

$53.16 \%$ 1,2-Dibromoethane

$0.04 \%$ Diethyl Phthalate

99.28\% Hexachlorobutadiene $5.93 \%$ Lindane

23.37\% 4-Methyl-2-Pentanone $60.12 \%$ Naphthalene $1.06 \%$ Pyrene

83.04\% 1,2,4-Trichlorobenzene $83.04 \%$ 1,2,4-Trichlorobenzene
44.56\% 1,2,3-Trichloropropane $91.37 \%$ Toluene $12.26 \% \mathrm{Hg}$ $0 \% \mathrm{HgO}$

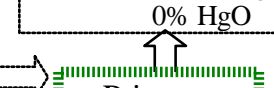
Primary Condenser

\section{Figure A-1. Organic}

Partitioning for Offgas

Regulatory Run UF1 Filtrate

1.22 density 8

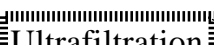
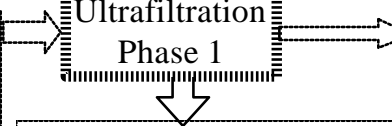

UF1 Slurry

$0.31 \%$ Aldrine

$0 \%$ Benzene

1.24\% Bis(Ethylhexyl)Phthalate 99.95\% Benzo(A)Pyrene 2.17\% Phenol

$0.20 \%$ Pentachlorophenol

99.89\% Hexachlorobenzene

$0 \%$ Chlorobenzene

$0 \%$ 2-Chloronaphthalene

2.16\% Dibenz[A,H]Anthracene

0\% 1,2-Dibromoethane

$0.48 \%$ Diethyl Phthalate

$0 \%$ Hexachlorobutadien

$0.01 \%$ Lindane

0\% 4-Methyl2-Pentanone

$0 \%$ Naphthalene

$0.08 \%$ Pyrene

$0 \%$ 1,2,4-Trichlorobenzene

0\%1,2,3-Trichloropropane $0 \%$ Toluene $0 \% \mathrm{HG}$

\section{PC Control Superheated \\ IC Vapor
$0.07 \%$ Aldrine}

Air PC Out Steam

$\coprod_{P C}+$ Ctrl

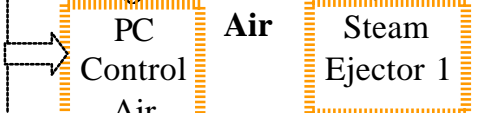

Air

Mixer

Steam

Ejector 1

Out

\section{PC Condensate}

85.44\% Aldrine

42.69\% Bis(Ethylhexyl)Phthalate

$0 \%$ Benzo(A)Pyrene

$0.15 \%$ Phenol

90.69\% Pentachlorophenol

0\% Hexachlorobenzene

17.4\% Chlorobenzene

49.14\%2-Chloronaphthalene

0.22\% Dibenz[A,H]Anthracene

$46.82 \%$ 1,2-Dibromoethane

78.02\% Diethyl Phthalate

$0.72 \%$ Hexachlorobutadiene 93.64\% Lindane

76.56\% 4-Methyl-2-Pentanone

$39.86 \%$ Naphthalene

95.06\% Pyrene

$16.96 \%$ 1,2,4-Trichlorobenzene

$55.42 \% 1,2,3$-Trichloropropane $8.63 \%$ Toluene $87.73 \% \mathrm{HG}$ $0 \% \mathrm{HgO}$

\section{UF1 Filtrate \\ $14.03 \%$ Aldrine}

0\% Benzene

$6.06 \%$ Bis(Ethylhexyl)Phthalat

$0.05 \%$ Benzo(A)Pyrene

97.68\% Phenol

8.97\% Pentachlorophenol

$0.11 \%$ Hexachlorobenzene

$0.01 \%$ Chlorobenzene

$0.02 \%$ 2-Chloronaphthalene

97.61\% Dibenz[A,H]Anthracene

0.02\% 1,2-Dibromoethane

$21.47 \%$ Diethyl Phthalate

$0 \%$ Hexachlorobutadiene $0.43 \%$ Lindane

0.07\% 4-Methyl-2-Pentanone

$0.02 \%$ Naphthalene $3.79 \%$ Pyrene

$0 \%$ 1,2,4-Trichlorobenzene

$0.03 \%$ 1,2,3-Trichloropropane

$0 \%$ Toluene

$0 \% \mathrm{Hg}$

$56.91 \% \mathrm{HgO}$
$90.01 \%$ Benzene

0\% Bis(Ethylhexyl)Phthalate

0\% Benzo(A)Pyrene

$0 \%$ Phenol

$0.01 \%$ Pentachlorophenol

$0 \%$ Hexachlorobenzene

82.19\% Chlorobenzene

49.76\% 2-Chloronaphthalene

0\% Dibenz[A,H]Anthracene

$52.1 \%$ 1,2-Dibromoethane

$0 \%$ Diethyl Phthalate

99.27\% Hexachlorobutadiene 4.43\% Lindane

21.73\% 4-Methyl-2-Pentanone

$59.22 \%$ Naphthalene $0.56 \%$ Pyrene

$82.66 \%$ 1,2,4-Trichlorobenzene

43.32\% 1,2,3-Trichloropropane 91.17\% Toluene $5.37 \% \mathrm{Hg}$ $0 \% \mathrm{HgO}$

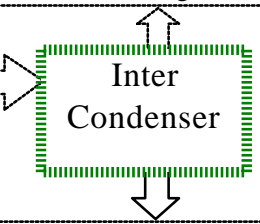

$0.15 \%$ Aldrine

$0.22 \%$ Benzene

$0.01 \%$ Bis(Ethylhexyl)Phthalat 0\% Benzo(A)Pyrene $0 \%$ Phenol

0\% Pentachlorophenol

0\% Hexachlorobenzene

$0.40 \%$ Chlorobenzene

1.08\% 2-Chloronaphthalene

$0 \%$ Dibenz[A,H]Anthracene

1.06\% 1,2-Dibromoethan

$0.04 \%$ Diethyl Phthalate

$0.01 \%$ Hexachlorobutadiene 1.49\% Lindane

1.64\% 4-Methyl-2-Pentanone

$0.90 \%$ Naphthalene

$0.50 \%$ Pyrene

$0.38 \%$ 1,2,4-Trichlorobenzene

$1.24 \% 1,2,3$-Trichloropropane

$0.20 \%$ Toluene

$0 \% \mathrm{HgO}$

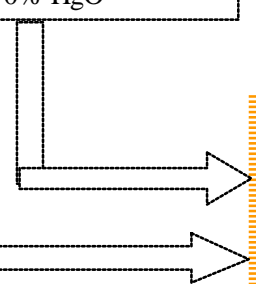

Superheated

Steam

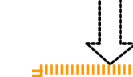

谙Ejector 2 Steam

0\% Bis(Ethylhexyl)Phthalat

0\% Benzo(A)Pyrene

$0 \%$ Phenol

Steam

Fiector

$\%$ Pentachlorophenol

$\%$ Hexachlorobenzene

80.87\% Chlorobenzene

46.38\% 2-Chloronaphthalene

0\% Dibenz[A,H]Anthracene

48.76\% 1,2-Dibromoethane

$0 \%$ Diethyl Phthalate

$99.23 \%$ Hexachlorobutadiene $2.08 \%$ Lindane

$17.33 \%$ 4-Methyl-2-Pentanone

$56.35 \%$ Naphthalene

$0.14 \%$ Pyrene

$81.4 \%$ 1,2,4Trichlorobenzene

$39.51 \%$ 1,2,3-Trichloropropane

90.5\% Toluene

$1.53 \% \mathrm{Hg}$

$0 \% \mathrm{HgO}$

After

Condenser

$$
\text { L }
$$

\section{AC Condensate \\ $0.06 \%$ Aldrine \\ $0.75 \%$ Benzene}

0\% Bis(Ethylhexyl)Phthalate

$$
0 \% \text { Phenol }
$$

$0.01 \%$ Pentachlorophenol

$0 \%$ Hexachlor

$1.32 \%$ Chlorobenzene

$3.39 \%$ 2-Chloronaphthalene

0\% Dibenz[A,H]Anthracene

3.34\% 1,2-Dibromoethane

$0 \%$ Diethyl Phthalate

$0.04 \%$ Hexachlorobutadiene $2.35 \%$ Lindane

$4.41 \%$ 4-Methyl-2-Pentanone

$2.87 \%$ Naphthalene

$0.43 \%$ Pyrene

$1.26 \%$ 1,2,4-Trichlorobenzene

$0.66 \%$ Toluene

$3.84 \% \mathrm{Hg}$

$0 \% \mathrm{HgO}$
U2-00550, Kev.

SRT-RPP-2002-0027

Demist Vapor

$0.01 \%$ Aldrine

0\% Bis(Ethylhexyl)Phthalate

$0 \%$ Benzo(A)Pyrene $0 \%$ Phenol

0\% Pentachlorophenol

$0 \%$ Hexachlorobenzene

80.87\% Chlorobenzene

46.37\% 2-Chloronaphthalene

0\% Dibenz[A,H]Anthracene

$48.76 \%$ 1 2-Dibromoethane

0\% Diethyl Phthalate

99.23\% Hexachlorobutadiene $2.08 \%$ Lindane

17.32\% 4-Methyl-2-Pentanon

$56.34 \%$ Naphthalene

$0.13 \%$ Pyrene

$81.4 \%$ 1,2,4Trichlorobenzene

39.51\% 1,2,3-Trichloropropan 90.5\% Toluene $1.22 \% \mathrm{Hg}$ $0 \% \mathrm{HgO}$ 广र

AC Demister 咅

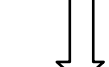

Demist liquid $0 \%$ Aldrine

$0 \%$ Benzene

$0 \%$ Bis(Ethylhexyl)Phthalate

0\% Benzo(A)Pyrene $0 \%$ Phenol

0\% Pentachlorophenol

0\% Hexachlorobenzene

0\% Chlorobenzene

$0.01 \% 2$-Chloronaphthalene

0\% Dibenz[A,H]Anthracene

$0 \%$ 1,2-Dibromoethane

$0 \%$ Diethyl Phthalate

0\% Hexachlorobutadiene $0 \%$ Lindane

\% 4-Methyl2-Pentanone $0 \%$ Naphthalene

$0 \%$ 1,2,4-Trichlorobenzene

$0 \% 1,2,3$-Trichloropropane $0 \%$ Toluene $0.32 \% \mathrm{Hg}$

$0 \% \mathrm{HgO}$ 
WSRC'-'IR-2UUZ-U055U, Kev. I

SRT-RPP-2002-0027.

Table A- 1. Waste Feed Evaporator Offgas Model Output for UF1 Filtrate Density of $1.22 \mathrm{~g} / \mathrm{ml}^{8}$

\begin{tabular}{|c|c|c|c|c|c|c|c|c|c|}
\hline Stream & $\begin{array}{l}\text { HLW OG } \\
\text { Cond }\end{array}$ & $\begin{array}{c}\text { Total } \\
\text { Recycle }\end{array}$ & Evap Feed & $\begin{array}{l}\text { EVAP } \\
\text { REDRXN } \\
\text { OUT }\end{array}$ & $\begin{array}{c}\text { Air } \\
\text { Inleakage }\end{array}$ & $\begin{array}{c}\text { Evap } \\
\text { Contents }\end{array}$ & Overhead & $\begin{array}{c}\text { Evap } \\
\text { Bottoms }\end{array}$ & $\begin{array}{c}\text { Cooled } \\
\text { Evap Bott }\end{array}$ \\
\hline Phase & Mixed & Mixed & Mixed & Mixed & Vapor & Mixed & Vapor & Mixed & Mixed \\
\hline Temperature, C & 25 & 25 & 25 & 50 & 25 & 50 & 50 & 50 & 25 \\
\hline Pressure, atm & 1 & 1 & 1 & 0.108335 & 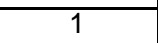 & 0.108583 & 0.108583 & 0.108583 & 1 \\
\hline $\mathrm{pH}$ & 11.2694 & 13.4748 & 13.0705 & 12.6538 & & 12.6388 & & 12.6388 & 13.5083 \\
\hline Total $\mathrm{mol} / \mathrm{hr}$ & 188132 & 4402.02 & 197463 & 390030 & 135.18 & 390165 & 268642 & 121522 & 121511 \\
\hline Flow Units & $\mathrm{g} / \mathrm{hr}$ & $\mathrm{g} / \mathrm{hr}$ & $\mathrm{g} / \mathrm{hr}$ & $\mathrm{g} / \mathrm{hr}$ & $\mathrm{g} / \mathrm{hr}$ & $\mathrm{g} / \mathrm{hr}$ & $\mathrm{g} / \mathrm{hr}$ & $\mathrm{g} / \mathrm{hr}$ & $\mathrm{g} / \mathrm{hr}$ \\
\hline Acetic Acid, Dimer & & & & $1.430 \mathrm{E}-18$ & & $1.440 \mathrm{E}-18$ & $1.440 \mathrm{E}-18$ & & \\
\hline Acetic Acid & & $8.809 \mathrm{E}-01$ & & $2.647 \mathrm{E}-06$ & & $2.643 \mathrm{E}-06$ & $2.643 \mathrm{E}-06$ & & \\
\hline Aluminum Oxide & $1.231 \mathrm{E}+00$ & $1.567 \mathrm{E}+02$ & $2.657 \mathrm{E}+03$ & $4.810 \mathrm{E}+03$ & & $4.810 \mathrm{E}+03$ & & $4.810 \mathrm{E}+03$ & $4.243 \mathrm{E}+03$ \\
\hline Aldrin & & $1.568 E-04$ & \begin{tabular}{|l|}
$4.195 E+00$ \\
\end{tabular} & \begin{tabular}{|l|}
$4.195 E+00$ \\
\end{tabular} & & $4.195 E+00$ & $3.593 E+00$ & $6.018 E-01$ & $6.018 E-01$ \\
\hline Aluminum Hydrogen EDTA & & & $1.741 \mathrm{E}+03$ & $1.190 \mathrm{E}+03$ & & $1.190 \mathrm{E}+03$ & & $1.190 \mathrm{E}+03$ & $1.429 \mathrm{E}+03$ \\
\hline Aluminum Hydroxide & & $6.264 \mathrm{E}+01$ & $3.103 \mathrm{E}+03$ & & & & & & $7.799 \mathrm{E}+02$ \\
\hline Benzene & & $7.739 E-07$ & \begin{tabular}{|l|}
$4.194 E+00$ \\
\end{tabular} & $4.194 E+00$ & & $4.194 E+00$ & $4.193 E+00$ & $1.189 E-04$ & $1.189 E-04$ \\
\hline Bis(2-ethylhexyl)phthalate (BEHP) & & $1.971 E-03$ & $4.193 E+00$ & $4.195 E+00$ & & $4.195 E+00$ & $1.791 E+00$ & $2.404 E+00$ & $2.404 E+00$ \\
\hline Benzo(a)pyrene (BaP) & & 9.236E-06 & $4.181 E+00$ & $4.181 E+00$ & & $4.181 E+00$ & & $4.181 E+00$ & $4.181 E+00$ \\
\hline Phenol & & $2.747 E-02$ & $4.196 E+00$ & $4.223 E+00$ & & $4.223 E+00$ & $6.495 E-03$ & $4.217 E+00$ & $4.217 E+00$ \\
\hline Calcium Chloride Oxide & $5.455 \mathrm{E}+02$ & & & & & & & & \\
\hline Calcium Oxalate & $6.886 \mathrm{E}+00$ & & $6.736 \mathrm{E}+02$ & $2.279 \mathrm{E}+02$ & & $2.251 \mathrm{E}+02$ & & $2.251 \mathrm{E}+02$ & $4.127 \mathrm{E}+02$ \\
\hline Calcium Oxalate Monohydrate & $2.059 \mathrm{E}+00$ & & & & & & & & \\
\hline Calcium Carbonate & & $2.337 \mathrm{E}+00$ & & & & & & & \\
\hline Calcium Dichromate (VI) & & $2.770 \mathrm{E}+00$ & & & & & & & \\
\hline Calcium Fluoride & $5.358 \mathrm{E}+02$ & & $3.237 \mathrm{E}+02$ & $1.649 \mathrm{E}+03$ & & $1.651 \mathrm{E}+03$ & & $1.651 \mathrm{E}+03$ & $1.536 \mathrm{E}+03$ \\
\hline Pentachlorophenol & & $2.372 E-03$ & $4.196 E+00$ & $4.199 E+00$ & & $4.199 E+00$ & $3.814 E+00$ & $3.848 E-01$ & $3.848 E-01$ \\
\hline Hexachlorobenzene & & $2.205 E-05$ & \begin{tabular}{|l|}
$4.188 E+00$ \\
\end{tabular} & $4.188 E+00$ & & $4.188 E+00$ & & $4.188 E+00$ & $4.188 E+00$ \\
\hline Chlorob & & $1.660 \mathrm{E}-06$ & $4.190 E+00$ & $4.190 E+00$ & & $4.190 E+00$ & $4.190 E+00$ & $2.554 E-04$ & $2.554 E-04$ \\
\hline 2-Chlorona & & $4.459 \mathrm{E}-06$ & \begin{tabular}{|l|}
$4.194 E+00$ \\
\end{tabular} & $4.194 E+00$ & & $4.194 E+00$ & $4.193 E+00$ & $7.931 E-04$ & $7.931 E-04$ \\
\hline Carbon Dioxide & & $5.136 \mathrm{E}+02$ & $7.847 \mathrm{E}+04$ & $7.899 \mathrm{E}+04$ & $949 \mathrm{E}+00$ & $7.899 \mathrm{E}+04$ & $2.500 \mathrm{E}-01$ & $7.899 \mathrm{E}+04$ & $7.899 \mathrm{E}+04$ \\
\hline Cesium Acetate & & $1.293 \mathrm{E}+00$ & $1.972 \mathrm{E}+02$ & \begin{tabular}{|l|}
$1.985 \mathrm{E}+02$ \\
\end{tabular} & & $1.985 \mathrm{E}+02$ & & $1.985 \mathrm{E}+02$ & $1.985 \mathrm{E}+02$ \\
\hline Dibenz[a,h]anthracene & & $1.102 E-03$ & $4.191 E+00$ & 4.192E+00 & & $4.192 E+00$ & $9.406 E-03$ & $4.182 E+00$ & $4.182 E+00$ \\
\hline 1,2-dibromoethane & & $3.989 E-05$ & $2.806 E+01$ & $2.806 E+01$ & & $2.806 E+01$ & $2.806 E+01$ & $6.127 E-03$ & $6.127 E-03$ \\
\hline Diethyl phthalate & & $5.992 E-03$ & $4.202 E+00$ & $4.208 E+00$ & & $4.208 E+00$ & $3.284 E+00$ & $9.234 E-01$ & $9.234 E-01$ \\
\hline Iron (III) Chloride & $1.159 \mathrm{E}+00$ & & & & & & & & \\
\hline Iron (III) Oxalate & & $3.728 \mathrm{E}-02$ & & & & & & & \\
\hline Iron (III) Hydrogen EDTA & & $1.097 \mathrm{E}+01$ & & $6.074 \mathrm{E}+02$ & & $6.074 \mathrm{E}+02$ & & $6.074 \mathrm{E}+02$ & $3.388 \mathrm{E}+02$ \\
\hline Iron (III) Hydroxide & $1.547 \mathrm{E}+02$ & $2.919 \mathrm{E}+01$ & & & & & & & $8.318 \mathrm{E}+01$ \\
\hline Formic Acid, dimer & & & & $7.040 \mathrm{E}-25$ & & $7.088 \mathrm{E}-25$ & $7.088 \mathrm{E}-25$ & & \\
\hline Hydrofluoride, dimer & & & & \begin{tabular}{|l}
$2.008 \mathrm{E}-22$ \\
\end{tabular} & & \begin{tabular}{l|}
$2.039 \mathrm{E}-22$ \\
\end{tabular} & $2.039 \mathrm{E}-22$ & & \\
\hline 2,2'-Iminobisacetic acid & & $7.425 \mathrm{E}+00$ & $1.132 \mathrm{E}+03$ & $1.140 \mathrm{E}+03$ & & $1.140 \mathrm{E}+03$ & & $1.140 \mathrm{E}+03$ & $1.140 \mathrm{E}+03$ \\
\hline Water & $3.361 \mathrm{E}+06$ & $7.570 \mathrm{E}+04$ & $3.290 \mathrm{E}+06$ & $6.727 \mathrm{E}+06$ & $1.819 \mathrm{E}+01$ & $6.727 \mathrm{E}+06$ & $4.837 \mathrm{E}+06$ & $1.890 \mathrm{E}+06$ & $1.891 \mathrm{E}+06$ \\
\hline \multicolumn{10}{|l|}{ Sulfuric Acid } \\
\hline Hydrogen Chloride & $1.862 \mathrm{E}+04$ & $7.852 \mathrm{E}+01$ & & $1.035 \mathrm{E}+04$ & & $1.035 \mathrm{E}+04$ & $4.604 \mathrm{E}-11$ & $1.035 \mathrm{E}+04$ & $1.035 \mathrm{E}+04$ \\
\hline Formic Acid & & $2.597 \mathrm{E}+00$ & $3.961 \mathrm{E}+02$ & $3.987 \mathrm{E}+02$ & & $3.987 \mathrm{E}+02$ & 2.927E-07 & $3.987 \mathrm{E}+02$ & $3.987 \mathrm{E}+02$ \\
\hline Hydrofluoric Acid & $2.116 \mathrm{E}+01$ & $1.559 \mathrm{E}+01$ & $3.388 \mathrm{E}+03$ & $3.020 \mathrm{E}+03$ & & $3.019 \mathrm{E}+03$ & $1.686 \mathrm{E}-07$ & $3.019 \mathrm{E}+03$ & $3.077 \mathrm{E}+03$ \\
\hline Mercury (II) Chloride & & & & \begin{tabular}{|l|}
$2.220 \mathrm{E}-07$ \\
\end{tabular} & & $2.240 \mathrm{E}-07$ & $2.240 \mathrm{E}-07$ & & \\
\hline Mercury (elemental) & & $3.801 \mathrm{E}-06$ & & \begin{tabular}{|l|}
$6.349 \mathrm{E}+01$ \\
\end{tabular} & & $6.349 \mathrm{E}+01$ & $6.349 \mathrm{E}+01$ & $5.835 \mathrm{E}-04$ & $5.835 \mathrm{E}-04$ \\
\hline Mercury (II) Oxide & & $9.851 \mathrm{E}-01$ & $1.361 \mathrm{E}+02$ & $6.850 \mathrm{E}+01$ & & $6.850 \mathrm{E}+01$ & & $6.850 \mathrm{E}+01$ & $6.850 \mathrm{E}+01$ \\
\hline Nitrous (III) Acid & $3.708 \mathrm{E}+03$ & $9.338 \mathrm{E}+02$ & $9.353 \mathrm{E}+04$ & $9.748 \mathrm{E}+04$ & & $9.766 \mathrm{E}+04$ & $8.501 \mathrm{E}-03$ & $9.766 \mathrm{E}+04$ & $9.342 \mathrm{E}+04$ \\
\hline Nitric Acid & $1.888 \mathrm{E}+03$ & $2.317 \mathrm{E}+03$ & $4.511 \mathrm{E}+04$ & $6.417 \mathrm{E}+04$ & & $6.417 \mathrm{E}+04$ & $1.712 \mathrm{E}-11$ & $6.417 \mathrm{E}+04$ & $6.417 \mathrm{E}+04$ \\
\hline Hexachlorobutadien & & $3.470 E-08$ & $4.200 E+00$ & $4.200 E+00$ & & $4.200 E+00$ & $4.200 E+00$ & $1.133 E-05$ & $1.133 E-05$ \\
\hline Potassium Citrate & & $1.851 \mathrm{E}+01$ & \begin{tabular}{|l|}
$2.823 \mathrm{E}+03$ \\
\end{tabular} & $2.842 \mathrm{E}+03$ & & $2.842 \mathrm{E}+03$ & & $2.842 \mathrm{E}+03$ & $2.842 \mathrm{E}+03$ \\
\hline Potassium Chloride & $1.845 \mathrm{E}+04$ & $2.222 \mathrm{E}+02$ & $1.350 \mathrm{E}+03$ & $3.760 \mathrm{E}+04$ & & $3.760 \mathrm{E}+04$ & & $3.760 \mathrm{E}+04$ & $3.760 \mathrm{E}+04$ \\
\hline Potassium Glycolate & & $3.479 \mathrm{E}+01$ & & & & & & & \\
\hline \multicolumn{10}{|l|}{ Potassium Nitrate $(\mathrm{VI})$} \\
\hline gamma-BHC (Lindane) & & 9.689E-05 & $4.209 E+00$ & $4.209 E+00$ & & $4.209 E+00$ & $4.190 E+00$ & $1.845 E-02$ & $1.845 E-02$ \\
\hline 4-methyl-2-pentanone (MIBK) & & $1.850 E-05$ & $4.197 E+00$ & 4.197E+00 & & $4.197 E+00$ & $4.194 E+00$ & $2.840 E-03$ & $2.840 \mathrm{E}-03$ \\
\hline Magnesium Oxalate & $2.060 \mathrm{E}+00$ & & & \begin{tabular}{|l}
$2.879 \mathrm{E}-02$ \\
\end{tabular} & & $2.947 \mathrm{E}-02$ & & $2.947 \mathrm{E}-02$ & $1.594 \mathrm{E}-02$ \\
\hline Magnesium Dichromate $(\mathrm{VI})$ & & $1.612 \mathrm{E}-04$ & & & & & & & \\
\hline
\end{tabular}


WSRC'-'IR-2UUZ-U055U, Rev. I

SRT-RPP-2002-0027.

\begin{tabular}{|c|c|c|c|c|c|c|c|c|c|}
\hline Stream & $\begin{array}{l}\text { HLW OG } \\
\text { Cond }\end{array}$ & $\begin{array}{c}\text { Total } \\
\text { Recycle }\end{array}$ & Evap Feed & $\begin{array}{c}\text { EVAP } \\
\text { REDRXN } \\
\text { OUT }\end{array}$ & $\begin{array}{c}\text { Air } \\
\text { Inleakage }\end{array}$ & $\begin{array}{c}\text { Evap } \\
\text { Contents }\end{array}$ & Overhead & $\begin{array}{c}\text { Evap } \\
\text { Bottoms }\end{array}$ & $\begin{array}{c}\text { Cooled } \\
\text { Evap Bott }\end{array}$ \\
\hline Magnesium Hydroxide & \begin{tabular}{|l|}
$2.368 \mathrm{E}+02$ \\
\end{tabular} & $6.448 \mathrm{E}-01$ & & $2.385 \mathrm{E}+02$ & & $2.385 \mathrm{E}+02$ & & $2.385 \mathrm{E}+02$ & $2.385 \mathrm{E}+02$ \\
\hline Manganese (II) Oxalate & & $1.182 \mathrm{E}-01$ & & $1.319 \mathrm{E}+00$ & & $1.319 \mathrm{E}+00$ & & $1.319 \mathrm{E}+00$ & $1.319 \mathrm{E}+00$ \\
\hline Manganese (II) Hydroxide & & $7.470 \mathrm{E}-01$ & & & & & & & \\
\hline Nitrogen & & $3.815 \mathrm{E}-06$ & & 3.815E-06 & $2.970 \mathrm{E}+03$ & $2.970 \mathrm{E}+03$ & $2.970 \mathrm{E}+03$ & $5.856 \mathrm{E}-04$ & $5.856 \mathrm{E}-04$ \\
\hline Sodium Oxalate & & $2.103 \mathrm{E}+00$ & $1.433 \mathrm{E}+04$ & $1.381 \mathrm{E}+04$ & & $1.382 \mathrm{E}+04$ & & $1.382 \mathrm{E}+04$ & $1.362 \mathrm{E}+04$ \\
\hline Sodium Chromate (VI) & & $5.125 \mathrm{E}+01$ & $8.349 \mathrm{E}+03$ & $8.404 \mathrm{E}+03$ & & $8.404 \mathrm{E}+03$ & & $8.404 \mathrm{E}+03$ & $8.404 \mathrm{E}+03$ \\
\hline Sodium Acetate & & & $1.835 \mathrm{E}+02$ & $1.847 \mathrm{E}+02$ & & $1.847 \mathrm{E}+02$ & & $1.847 \mathrm{E}+02$ & $1.847 \mathrm{E}+02$ \\
\hline Sodium Glycolate & & & $4.556 \mathrm{E}+03$ & $4.586 \mathrm{E}+03$ & & $4.586 \mathrm{E}+03$ & & $4.586 \mathrm{E}+03$ & $4.586 \mathrm{E}+03$ \\
\hline Sodium Nitrite & $3.054 \mathrm{E}+03$ & & $6.327 \mathrm{E}+04$ & $6.731 \mathrm{E}+04$ & & $6.704 \mathrm{E}+04$ & & $6.704 \mathrm{E}+04$ & $7.327 \mathrm{E}+04$ \\
\hline Sodium Hydroxide & $2.572 E+04$ & $4.789 \mathrm{E}+03$ & $3.253 \mathrm{E}+05$ & $3.554 \mathrm{E}+05$ & & $3.555 \mathrm{E}+05$ & & $3.555 \mathrm{E}+05$ & $3.520 \mathrm{E}+05$ \\
\hline Naphthalene & & 4.906E-06 & $4.188 E+00$ & $4.188 E+00$ & & $4.188 E+00$ & +00 & 7.733E-04 & 7.733E-04 \\
\hline Sodium Aluminosilicate Gel & $2.920 \mathrm{E}+03$ & $8.103 \mathrm{E}+01$ & & $3.617 \mathrm{E}+03$ & & $3.618 \mathrm{E}+03$ & & $3.618 \mathrm{E}+03$ & $3.690 \mathrm{E}+03$ \\
\hline Nickel (II) Oxalate & & $3.977 \mathrm{E}+00$ & & $3.977 \mathrm{E}+00$ & & $3.977 \mathrm{E}+00$ & & $3.977 \mathrm{E}+00$ & $3.977 \mathrm{E}+00$ \\
\hline Oxygen & & $2.122 \mathrm{E}-06$ & & $2.122 \mathrm{E}-06$ & $8.994 \mathrm{E}+02$ & $8.994 \mathrm{E}+02$ & 8.994E+02 & $3.258 \mathrm{E}-04$ & $3.258 \mathrm{E}-04$ \\
\hline Phosphorus Pentoxide & & $4.191 \mathrm{E}+00$ & $6.391 \mathrm{E}+02$ & $6.433 \mathrm{E}+02$ & & $6.433 \mathrm{E}+02$ & & $6.433 \mathrm{E}+02$ & $6.433 \mathrm{E}+02$ \\
\hline PYRENE & & $1.524 E-04$ & $4.202 E+00$ & $4.202 E+00$ & & $4.202 E+00$ & $4.039 E+00$ & $1.629 E-01$ & $1.629 E-01$ \\
\hline Silicon Dioxide & $3.339 \mathrm{E}+02$ & $3.561 \mathrm{E}+00$ & & $1.142 \mathrm{E}+02$ & & $1.141 \mathrm{E}+02$ & & $1.141 \mathrm{E}+02$ & $8.786 \mathrm{E}+01$ \\
\hline Sulfur Trioxide & \begin{tabular}{|l|}
$5.781 E+02$ \\
\end{tabular} & $2.021 \mathrm{E}+02$ & $4.702 \mathrm{E}+04$ & $4.780 \mathrm{E}+04$ & & $4.780 \mathrm{E}+04$ & & $4.780 \mathrm{E}+04$ & $4.780 \mathrm{E}+04$ \\
\hline 1,2,4-trichlorobenzene & & $1.035 E-06$ & $4.193 E+00$ & $4.193 E+00$ & & $4.193 E+00$ & $4.193 E+00$ & $1.800 E-04$ & $1.800 \mathrm{E}-04$ \\
\hline 1,2,3-trichloropropane & & $7.301 E-06$ & $4.198 E+00$ & $4.198 E+00$ & & $4.198 E+00$ & $4.197 E+00$ & $1.123 E-03$ & $1.123 E-03$ \\
\hline TOLUENE & & $6.433 E-07$ & $4.194 E+00$ & $4.194 E+00$ & & $4.194 E+00$ & $4.194 E+00$ & $9.908 E-05$ & $9.908 E-05$ \\
\hline Zinc Dichloride Pentoxide & $9.198 \mathrm{E}+00$ & & & & & & & & \\
\hline Zinc Oxalate & & $7.471 \mathrm{E}+00$ & & $1.147 \mathrm{E}+03$ & & $1.147 \mathrm{E}+03$ & & $1.147 \mathrm{E}+03$ & $1.147 \mathrm{E}+03$ \\
\hline Zinc Hydroxide & $7.281 \mathrm{E}+02$ & & & & & & & & \\
\hline Zirconium EDTA & & $1.634 \mathrm{E}+00$ & & $6.821 \mathrm{E}+00$ & & $6.806 \mathrm{E}+00$ & & $6.806 \mathrm{E}+00$ & $1.525 \mathrm{E}+01$ \\
\hline Zirconium Oxide & & $4.421 \mathrm{E}+00$ & & $2.737 \mathrm{E}+00$ & & $2.742 \mathrm{E}+00$ & & $2.742 \mathrm{E}+00$ & \\
\hline Total $\mathrm{g} / \mathrm{hr}$ & $3.438 \mathrm{E}+06$ & $8.526 \mathrm{E}+04$ & $4.012 \mathrm{E}+06$ & $7.535 \mathrm{E}+06$ & $3.889 \mathrm{E}+03$ & $7.539 \mathrm{E}+06$ & $4.841 \mathrm{E}+06$ & $2.698 \mathrm{E}+06$ & $2.698 \mathrm{E}+06$ \\
\hline Volume, L/hr & \begin{tabular}{|l|}
$3.405 \mathrm{E}+03$ \\
\end{tabular} & $7.946 \mathrm{E}+01$ & $3.551 \mathrm{E}+03$ & $6.617 \mathrm{E}+07$ & \begin{tabular}{|l}
$3.307 \mathrm{E}+03$ \\
\end{tabular} & $6.552 \mathrm{E}+07$ & $6.552 \mathrm{E}+07$ & $2.252 \mathrm{E}+03$ & $2.203 \mathrm{E}+03$ \\
\hline Enthalpy, cal/hr & $\mid-1.289 \mathrm{E}+10$ & $-3.074 \mathrm{E}+08$ & $-1.404 \mathrm{E}+10$ & $-2.430 \mathrm{E}+10$ & $-6.275 \mathrm{E}+04$ & $-2.432 \mathrm{E}+10$ & $-1.547 \mathrm{E}+10$ & $-8.855 \mathrm{E}+09$ & $-8.904 \mathrm{E}+09$ \\
\hline Vapor fraction & & & & $6.940 \mathrm{E}-01$ & $1.000 \mathrm{E}+00$ & $6.885 \mathrm{E}-01$ & $\mathrm{E}+00$ & & \\
\hline Solid fraction & 1.181E-04 & $2.782 \mathrm{E}-04$ & 2.237E-04 & \begin{tabular}{|l|}
$1.381 \mathrm{E}-04$ \\
\end{tabular} & & $1.316 \mathrm{E}-04$ & & $4.226 \mathrm{E}-04$ & $8.146 \mathrm{E}-04$ \\
\hline Organic fraction & & $9.595 \mathrm{E}-10$ & 4.886E-07 & $6.340 \mathrm{E}-08$ & & $6.354 \mathrm{E}-08$ & & $2.040 \mathrm{E}-07$ & 1.996E-07 \\
\hline Osmotic Pres, atm & $1.256 \mathrm{E}+01$ & $6.077 \mathrm{E}+01$ & $9.071 \mathrm{E}+01$ & $1.802 \mathrm{E}+02$ & & $1.774 \mathrm{E}+02$ & & $1.774 \mathrm{E}+02$ & $1.739 \mathrm{E}+02$ \\
\hline \multicolumn{10}{|l|}{ Redox Pot, volts } \\
\hline E-Con, 1/ohm-cm & $2.639 \mathrm{E}-02$ & $1.235 \mathrm{E}-01$ & $1.378 \mathrm{E}-01$ & $3.040 \mathrm{E}-01$ & & $1.860 \mathrm{E}-01$ & & $1.860 \mathrm{E}-01$ & $2.012 \mathrm{E}-01$ \\
\hline E-Con, cm2/ohm mol & $9.004 \mathrm{E}+01$ & $4.892 \mathrm{E}+01$ & $6.830 \mathrm{E}+01$ & $4.044 \mathrm{E}+01$ & & $2.497 \mathrm{E}+01$ & & $2.497 \mathrm{E}+01$ & $2.663 \mathrm{E}+01$ \\
\hline Abs Visc, cP & \begin{tabular}{|l|}
$9.062 \mathrm{E}-01$ \\
\end{tabular} & $1.150 \mathrm{E}+00$ & $1.494 \mathrm{E}+00$ & $1.444 \mathrm{E}+00$ & & $1.421 \mathrm{E}+00$ & & $1.421 \mathrm{E}+00$ & $2.328 \mathrm{E}+00$ \\
\hline Rel Visc & $1.017 \mathrm{E}+00$ & $1.291 \mathrm{E}+00$ & $1.678 \mathrm{E}+00$ & $2.639 \mathrm{E}+00$ & & $2.597 \mathrm{E}+00$ & & $2.597 \mathrm{E}+00$ & $2.613 \mathrm{E}+00$ \\
\hline Ionic Strength & \begin{tabular}{|l|}
$2.815 \mathrm{E}-01$ \\
\end{tabular} & $1.596 \mathrm{E}+00$ & \begin{tabular}{|l|}
$3.062 \mathrm{E}+00$ \\
\end{tabular} & $5.903 \mathrm{E}+00$ & & $5.791 \mathrm{E}+00$ & & $5.791 \mathrm{E}+00$ & $5.571 \mathrm{E}+00$ \\
\hline
\end{tabular}


Table A- 1. Waste Feed Evaporator Offgas Model Output for UF1 Filtrate Density of $1.22 \mathrm{~g} / \mathrm{ml}$ (cont'd) ${ }^{8}$

\begin{tabular}{|c|c|c|c|c|c|c|c|c|c|}
\hline Stream & UF1 Filtrate & UF1 Slurry & PC Vapor & \begin{tabular}{c|} 
PC \\
Condensate
\end{tabular} & $\begin{array}{c}\text { PC Control } \\
\text { Air }\end{array}$ & \begin{tabular}{|c|} 
PC Vap + \\
CtrlAir
\end{tabular} & $\begin{array}{c}\text { Super } \\
\text { Steam } 1\end{array}$ & $\begin{array}{c}\text { Stm Eject } 1 \\
\text { Out }\end{array}$ & IC Vapor \\
\hline Phase & Mixed & Mixed & Vapor & Mixed & Vapor & Vapor & Aqueous & Mixed & Vapor \\
\hline Temperature, C & 25 & 25 & 40 & 40 & 25 & 34.7136 & 133 & 58.2385 & 40 \\
\hline Pressure, atm & 1 & 1 & 0.103151 & 0.103151 & 1 & 0.0979798 & 8.41674 & 0.294007 & 0.279311 \\
\hline $\mathrm{pH}$ & 13.5083 & 13.5083 & & 6.64281 & & & 5.90373 & 6.24478 & \\
\hline Total $\mathrm{mol} / \mathrm{hr}$ & 118779 & 2732.49 & 460.579 & 268182 & 270.36 & 730.939 & 2517.81 & 3248.75 & 545.576 \\
\hline Flow Units & $\mathrm{g} / \mathrm{hr}$ & $\mathrm{g} / \mathrm{hr}$ & $\mathrm{g} / \mathrm{hr}$ & $\mathrm{g} / \mathrm{hr}$ & $\mathrm{g} / \mathrm{hr}$ & $\mathrm{g} / \mathrm{hr}$ & $\mathrm{g} / \mathrm{hr}$ & $\mathrm{g} / \mathrm{hr}$ & $\mathrm{g} / \mathrm{hr}$ \\
\hline Acetic Acid, Dimer & & & $1.318 \mathrm{E}-25$ & & & & & & \\
\hline Acetic Acid & & & $2.812 \mathrm{E}-11$ & $2.643 \mathrm{E}-06$ & & & & & \\
\hline Aluminum Oxide & $4.151 \mathrm{E}+03$ & $9.202 \mathrm{E}+01$ & & & & & & & \\
\hline Aldrin & $5.887 E-01$ & $1.305 E-02$ & $9.150 E-03$ & $3.584 E+00$ & & $9.150 E-03$ & & $9.150 E-03$ & $2.991 E-03$ \\
\hline Aluminum Hydrogen EDTA & $1.398 \mathrm{E}+03$ & $3.100 \mathrm{E}+01$ & & & & & & & \\
\hline Aluminum Hydroxide & & $7.799 \mathrm{E}+02$ & & & & & & & \\
\hline Benzene & $1.163 E-04$ & \begin{tabular}{|l|}
$2.579 E-06$ \\
\end{tabular} & $3.784 E+00$ & 4.094E-01 & & $3.784 E+00$ & & $3.784 E+00$ & $3.775 E+00$ \\
\hline Bis(2-ethylhexyl)phthalate (BEHP) & $2.352 E+00$ & $5.215 E-02$ & 3.377E-04 & $1.791 E+00$ & & 3.377E-04 & & \begin{tabular}{|l|}
$3.377 E-04$ \\
\end{tabular} & 1.127E-05 \\
\hline Benzo(a)pyrene (BaP) & $1.923 E-03$ & $4.180 E+00$ & & & & & & & \\
\hline Phenol & $4.125 E+00$ & $9.146 E-02$ & $1.045 E-05$ & $6.484 E-03$ & & $1.045 E-05$ & & $1.045 E-05$ & $6.855 E-07$ \\
\hline Calcium Chloride Oxide & & & & & & & & & \\
\hline Calcium Oxalate & $4.037 \mathrm{E}+02$ & $8.951 \mathrm{E}+00$ & & & & & & & \\
\hline Calcium Oxalate Monohydrate & & & & & & & & & \\
\hline Calcium Carbonate & & & & & & & & & \\
\hline Calcium Dichromate (VI) & & & & & & & & & \\
\hline Calcium Fluoride & & $1.536 \mathrm{E}+03$ & & & & & & & \\
\hline Pentachlorophenol & $3.764 E-01$ & $8.346 E-03$ & $6.081 E-03$ & $3.808 E+00$ & & $6.081 E-03$ & & \begin{tabular}{|l|}
$6.081 E-03$ \\
\end{tabular} & $4.004 E-04$ \\
\hline Hexachlorobenzene & $4.591 E-03$ & $4.183 E+00$ & & & & & & & \\
\hline Chlorobenzene & $2.498 E-04$ & \begin{tabular}{|l|}
$5.539 E-06$ \\
\end{tabular} & $3.461 E+00$ & $7.293 E-01$ & & $3.461 E+00$ & & $3.461 E+00$ & $3.444 E+00$ \\
\hline 2-Chloronaphthalene & $7.759 E-04$ & $1.720 E-05$ & $2.132 E+00$ & $2.061 E+00$ & & $2.132 E+00$ & & $2.132 E+00$ & $2.087 E+00$ \\
\hline Carbon Dioxide & $7.727 \mathrm{E}+04$ & $1.713 \mathrm{E}+03$ & $2.307 \mathrm{E}-01$ & $1.935 \mathrm{E}-02$ & $3.897 \mathrm{E}+00$ & $4.128 \mathrm{E}+00$ & & $4.128 \mathrm{E}+00$ & $4.124 \mathrm{E}+00$ \\
\hline Cesium Acetate & $1.942 E+02$ & $4.306 \mathrm{E}+00$ & & & & & & & \\
\hline Dibenz[a,h]anthracene & $4.092 E+00$ & \begin{tabular}{|l|}
$9.072 E-02$ \\
\end{tabular} & $7.586 \mathrm{E}-09$ & $9.406 \mathrm{E}-03$ & & \begin{tabular}{|l|}
$7.586 E-09$ \\
\end{tabular} & & $7.586 E-09$ & $1.333 E-12$ \\
\hline 1,2-dibromoethane & $5.994 E-03$ & \begin{tabular}{|l|}
$1.329 E-04$ \\
\end{tabular} & $1.492 E+01$ & $1.314 E+01$ & & $1.492 E+01$ & & $1.492 E+01$ & $1.462 E+01$ \\
\hline Diethyl phthalate & $9.033 E-01$ & $2.003 E-02$ & $1.571 E-03$ & $3.283 E+00$ & & $1.571 E-03$ & & $1.571 E-03$ & 3.211E-05 \\
\hline Iron (III) Chloride & & & & & & & & & \\
\hline Iron (III) Oxalate & & & & & & & & & \\
\hline Iron (III) Hydrogen EDTA & $3.314 \mathrm{E}+02$ & $7.349 \mathrm{E}+00$ & & & & & & & \\
\hline Iron (III) Hydroxide & & $8.318 \mathrm{E}+01$ & & & & & & & \\
\hline Formic Acid, dimer & & & & & & & & & \\
\hline Hydrofluoride, dimer & & & & & & & & & \\
\hline 2,2'-Iminobisacetic acid & $1.115 \mathrm{E}+03$ & $2.472 \mathrm{E}+01$ & & & & & & & \\
\hline Water & $1.850 \mathrm{E}+06$ & $4.102 \mathrm{E}+04$ & $5.877 \mathrm{E}+03$ & $4.831 \mathrm{E}+06$ & $3.638 \mathrm{E}+01$ & $5.913 \mathrm{E}+03$ & $4.536 \mathrm{E}+04$ & $5.127 \mathrm{E}+04$ & $2.574 \mathrm{E}+03$ \\
\hline Sulfuric Acid & & & & & & & & & \\
\hline Hydrogen Chloride & $1.012 \mathrm{E}+04$ & $2.244 \mathrm{E}+02$ & $3.362 \mathrm{E}-20$ & $6.020 \mathrm{E}-08$ & & & & & \\
\hline Formic Acid & $3.900 \mathrm{E}+02$ & $8.647 \mathrm{E}+00$ & $1.965 \mathrm{E}-13$ & $2.927 \mathrm{E}-07$ & & & & & \\
\hline Hydrofluoric Acid & $3.011 \mathrm{E}+03$ & $6.675 \mathrm{E}+01$ & $8.251 \mathrm{E}-15$ & $1.686 \mathrm{E}-07$ & & & & & \\
\hline Mercury (II) Chloride & & & & & & & & & \\
\hline Mercury (elemental) & $5.708 \mathrm{E}-04$ & 1.266E-05 & $7.786 \mathrm{E}+00$ & $5.570 \mathrm{E}+01$ & & $7.786 \mathrm{E}+00$ & & $7.786 \mathrm{E}+00$ & $3.408 \mathrm{E}+00$ \\
\hline Mercury (II) Oxide & $3.899 \mathrm{E}+01$ & $2.951 \mathrm{E}+01$ & & $1.787 \mathrm{E}-07$ & & & & & \\
\hline Nitrous (III) Acid & $9.139 \mathrm{E}+04$ & $2.026 \mathrm{E}+03$ & 5.316E-07 & $8.500 \mathrm{E}-03$ & & $5.316 \mathrm{E}-07$ & & $5.316 \mathrm{E}-07$ & $4.936 \mathrm{E}-09$ \\
\hline Nitric Acid & $6.278 \mathrm{E}+04$ & $1.392 \mathrm{E}+03$ & & & & & & & \\
\hline Hexachlorobutadiene & 1.109E-05 & \begin{tabular}{|l|}
$2.458 E-07$ \\
\end{tabular} & $4.170 E+00$ & $3.005 E-02$ & & $4.170 E+00$ & & $4.170 E+00$ & $4.169 E+00$ \\
\hline Potassium Citrate & $2.780 \mathrm{E}+03$ & $6.164 \mathrm{E}+01$ & & & & & & & \\
\hline Potassium Chloride & $3.678 \mathrm{E}+04$ & $8.155 \mathrm{E}+02$ & & & & & & & \\
\hline Potassium Glycolate & & & & & & & & & \\
\hline Potassium Nitrate $(\mathrm{VI})$ & & & & & & & & & \\
\hline gamma-BHC (Lindane) & $1.805 E-02$ & \begin{tabular}{|l|}
$4.003 E-04$ \\
\end{tabular} & $2.494 E-01$ & $3.941 E+00$ & & \begin{tabular}{|l|}
$2.494 E-01$ \\
\end{tabular} & & $2.494 E-01$ & $1.866 E-01$ \\
\hline 4-methyl-2-pentanone (MIBK) & $2.779 E-03$ & \begin{tabular}{|l|}
$6.161 E-05$ \\
\end{tabular} & $9.808 \mathrm{E}-01$ & $3.213 E+00$ & & \begin{tabular}{|l|}
$9.808 E-01$ \\
\end{tabular} & & $9.808 E-01$ & $9.121 E-01$ \\
\hline Magnesium Oxalate & $1.559 \mathrm{E}-02$ & \begin{tabular}{|l|}
$3.457 \mathrm{E}-04$ \\
\end{tabular} & & & & & & & \\
\hline Magnesium Dichromate $(\mathrm{VI})$ & & & & & & & & & \\
\hline
\end{tabular}


WSRC-'IR-2UU2-00550, Rev. I

SRT-RPP-2002-0027.

\begin{tabular}{|c|c|c|c|c|c|c|c|c|c|}
\hline Stream & UF1 Filtrate & UF1 Slurry & PC Vapor & $\begin{array}{c}\mathrm{PC} \\
\text { Condensate }\end{array}$ & ${ }_{\text {Air }}^{P C \text { Control }}$ & $\begin{array}{c}\text { PC Vap + } \\
\text { CtrlAir }\end{array}$ & $\begin{array}{c}\text { Super } \\
\text { Steam } 1\end{array}$ & $\begin{array}{c}\text { Stm Eject } 1 \\
\text { Out }\end{array}$ & IC Vapor \\
\hline Magnesium Hydroxide & & $2.385 \mathrm{E}+02$ & & & & & & & \\
\hline Manganese (II) Oxalate & $1.290 \mathrm{E}+00$ & \begin{tabular}{|l|}
$2.860 \mathrm{E}-02$ \\
\end{tabular} & & & & & & & \\
\hline \multicolumn{10}{|l|}{ Manganese (II) Hydroxide } \\
\hline Nitrogen & $5.729 \mathrm{E}-04$ & $1.270 \mathrm{E}-05$ & $2.968 \mathrm{E}+03$ & $1.765 \mathrm{E}+00$ & $5.940 \mathrm{E}+03$ & $8.908 \mathrm{E}+03$ & & $8.908 \mathrm{E}+03$ & $8.908 \mathrm{E}+03$ \\
\hline Sodium Oxalate & $5.317 \mathrm{E}+03$ & $8.304 \mathrm{E}+03$ & & & & & & & \\
\hline Sodium Chromate (VI) & $8.222 \mathrm{E}+03$ & $1.823 \mathrm{E}+02$ & & & & & & & \\
\hline Sodium Acetate & $1.807 \mathrm{E}+02$ & $4.007 \mathrm{E}+00$ & & & & & & & \\
\hline Sodium Glycolate & $4.487 \mathrm{E}+03$ & $9.948 \mathrm{E}+01$ & & & & & & & \\
\hline Sodium Nitrite & $7.168 \mathrm{E}+04$ & $1.589 \mathrm{E}+03$ & & & & & & & \\
\hline Sodium Hydroxide & $3.444 \mathrm{E}+05$ & $7.636 \mathrm{E}+03$ & & & & & & & \\
\hline Naphthalene & $7.565 E-04$ & \begin{tabular}{|l|}
$1.677 E-05$ \\
\end{tabular} & $2.518 E+00$ & $1.670 E+00$ & & $2.518 E+00$ & & $2.518 E+00$ & $2.480 E+00$ \\
\hline Sodium Aluminosilicate Gel & & $3.690 \mathrm{E}+03$ & & & & & & & \\
\hline Nickel (II) Oxalate & $3.890 \mathrm{E}+00$ & \begin{tabular}{|l|}
$8.625 \mathrm{E}-02$ \\
\end{tabular} & & & & & & & \\
\hline Oxygen & $3.187 \mathrm{E}-04$ & 7.067E-06 & $8.984 \mathrm{E}+02$ & $1.002 \mathrm{E}+00$ & $1.799 \mathrm{E}+03$ & $2.697 \mathrm{E}+03$ & & $2.697 \mathrm{E}+03$ & $2.697 \mathrm{E}+03$ \\
\hline Phosphorus Pentoxide & $6.294 \mathrm{E}+02$ & \begin{tabular}{|l|}
$1.395 \mathrm{E}+01$ \\
\end{tabular} & & & & & & & \\
\hline PYRENE & $1.593 E-01$ & \begin{tabular}{|l|}
$3.532 E-03$ \\
\end{tabular} & $4.456 E-02$ & $3.994 E+00$ & & $4.456 E-02$ & & 4.456E-02 & $2.353 E-02$ \\
\hline Silicon Dioxide & $8.596 \mathrm{E}+01$ & $1.906 \mathrm{E}+00$ & & & & & & & \\
\hline Sulfur Trioxide & $4.676 \mathrm{E}+04$ & $1.037 \mathrm{E}+03$ & & & & & & & \\
\hline 1,2,4-trichlorobenzene & $1.761 E-04$ & \begin{tabular}{|l|}
$3.904 E-06$ \\
\end{tabular} & $3.482 E+00$ & $7.110 E-01$ & & $3.482 E+00$ & & $3.482 E+00$ & $3.466 E+00$ \\
\hline 1,2,3-trichloropropane & $1.098 E-03$ & 2.435E-05 & $1.871 E+00$ & $2.327 E+00$ & & $1.871 E+00$ & & $1.871 E+00$ & $1.819 E+00$ \\
\hline TOLUENE & $9.693 E-05$ & \begin{tabular}{|l|}
$2.149 E-06$ \\
\end{tabular} & $3.832 E+00$ & $3.620 E-01$ & & $3.832 E+00$ & & $3.832 E+00$ & $3.823 E+00$ \\
\hline \multicolumn{10}{|l|}{ Zinc Dichloride Pentoxide } \\
\hline Zinc Oxalate & $1.122 \mathrm{E}+03$ & $2.487 \mathrm{E}+01$ & & & & & & & \\
\hline \multicolumn{10}{|l|}{ Zinc Hydroxide } \\
\hline Zirconium EDTA & $1.492 \mathrm{E}+01$ & $3.308 \mathrm{E}-01$ & & & & & & & \\
\hline \multicolumn{10}{|l|}{ Zirconium Oxide } \\
\hline Total g/hr & $2.625 \mathrm{E}+06$ & $7.276 \mathrm{E}+04$ & $9.793 \mathrm{E}+03$ & $4.831 \mathrm{E}+06$ & $7.779 E+03$ & $1.757 \mathrm{E}+04$ & $4.536 \mathrm{E}+04$ & $6.293 \mathrm{E}+04$ & $1.423 \mathrm{E}+04$ \\
\hline Volume, L/hr & $2.152 \mathrm{E}+03$ & $5.145 \mathrm{E}+01$ & $1.146 \mathrm{E}+05$ & $4.870 \mathrm{E}+03$ & $6.613 \mathrm{E}+03$ & $1.884 \mathrm{E}+05$ & $4.866 \mathrm{E}+01$ & $9.736 \mathrm{E}+04$ & $5.017 \mathrm{E}+04$ \\
\hline Enthalpy, cal/hr & $-8.669 \mathrm{E}+09$ & $-2.350 E+08$ & $-1.880 \mathrm{E}+07$ & $-1.825 \mathrm{E}+10$ & $-1.255 \mathrm{E}+05$ & \begin{tabular}{|c|}
$-1.893 \mathrm{E}+07$ \\
\end{tabular} & $-1.671 \mathrm{E}+08$ & $-1.860 \mathrm{E}+08$ & $-8.208 \mathrm{E}+06$ \\
\hline Vapor fraction & & & $1.000 \mathrm{E}+00$ & & $1.000 \mathrm{E}+00$ & $1.000 \mathrm{E}+00$ & & $3.244 \mathrm{E}-01$ & $1.000 \mathrm{E}+00$ \\
\hline Solid fraction & & \begin{tabular}{|l|}
$3.622 \mathrm{E}-02$ \\
\end{tabular} & & $1.031 \mathrm{E}-06$ & & & & & \\
\hline Organic fraction & $1.997 \mathrm{E}-07$ & \begin{tabular}{|l|}
$1.925 \mathrm{E}-07$ \\
\end{tabular} & & $1.115 \mathrm{E}-07$ & & & & & \\
\hline Osmotic Pres, atm & $1.739 \mathrm{E}+02$ & \begin{tabular}{|l|}
$1.739 \mathrm{E}+02$ \\
\end{tabular} & & $1.628 \mathrm{E}-03$ & & & & $2.343 \mathrm{E}-03$ & \\
\hline \multicolumn{10}{|l|}{ Redox Pot, volts } \\
\hline E-Con, 1/ohm-cm & & & & $1.325 \mathrm{E}-07$ & & & $1.478 \mathrm{E}-06$ & $3.558 \mathrm{E}-07$ & \\
\hline E-Con, cm2/ohm-mol & & & & $4.780 \mathrm{E}-03$ & & & & $3.550 \mathrm{E}-05$ & \\
\hline Abs Visc, cP & & & & $6.536 \mathrm{E}-01$ & & & $2.077 \mathrm{E}-01$ & \begin{tabular}{|l|}
$4.792 \mathrm{E}-01$ \\
\end{tabular} & \\
\hline Rel Visc & & & & $1.000 \mathrm{E}+00$ & & & $1.000 \mathrm{E}+00$ & $1.000 \mathrm{E}+00$ & \\
\hline Tonic Strength & $5.571 \mathrm{E}+00$ & $5.571 \mathrm{E}+00$ & & $2.278 \mathrm{E}-07$ & & & $1.250 \mathrm{E}-06$ & \begin{tabular}{|l|}
$5.697 \mathrm{E}-07$ \\
\end{tabular} & \\
\hline
\end{tabular}


Table A- 1. Waste Feed Evaporator Offgas Model Output for UF1 Filtrate Density of $1.22 \mathrm{~g} / \mathrm{ml}$ (cont'd) ${ }^{8}$

\begin{tabular}{|c|c|c|c|c|c|c|c|c|}
\hline Stream & \begin{tabular}{c|} 
IC \\
Condensate
\end{tabular} & $\begin{array}{c}\text { Super } \\
\text { Steam } 2\end{array}$ & $\begin{array}{c}\text { Stm Eject } \\
2 \text { Out }\end{array}$ & AC Vapor & \begin{tabular}{c|}
$\mathbf{A C}$ \\
Condensate
\end{tabular} & $\begin{array}{l}\text { Demist } \\
\text { Vapor }\end{array}$ & $\begin{array}{l}\text { Demist } \\
\text { Liquid }\end{array}$ & $\begin{array}{l}\text { Evap Cond } \\
\text { Tk Out }\end{array}$ \\
\hline Phase & Mixed & Aqueous & Mixed & Vapor & Mixed & Mixed & Mixed & Mixed \\
\hline Temperature, $\mathrm{C}$ & 40 & 133 & 76.7327 & 40 & 40 & 40 & 40 & 25 \\
\hline Pressure, atm & 0.279311 & 8.41674 & \begin{tabular}{|l|}
0.837932 \\
\end{tabular} & 0.796018 & 0.796018 & 0.985242 & 0.985242 & 1 \\
\hline $\mathrm{pH}$ & 6.10493 & 5.90373 & 6.02944 & & 5.84094 & 5.7912 & 5.7912 & 6.70197 \\
\hline Total $\mathrm{mol} / \mathrm{hr}$ & 2703.18 & 2517.81 & 3063.39 & 443.532 & 2619.86 & 441.876 & 1.65655 & 273507 \\
\hline Flow Units & $\mathrm{g} / \mathrm{hr}$ & $\mathrm{g} / \mathrm{hr}$ & $\mathrm{g} / \mathrm{hr}$ & $\mathrm{g} / \mathrm{hr}$ & $\mathrm{g} / \mathrm{hr}$ & $\mathrm{g} / \mathrm{hr}$ & $\mathrm{g} / \mathrm{hr}$ & $\mathrm{g} / \mathrm{hr}$ \\
\hline \multicolumn{9}{|l|}{ Acetic Acid, Dimer } \\
\hline Acetic Acid & & & & & & & & $2.643 \mathrm{E}-06$ \\
\hline \multicolumn{9}{|l|}{ Aluminum Oxide } \\
\hline Aldrin & $6.159 E-03$ & & $2.991 E-03$ & $3.956 E-04$ & $2.596 E-03$ & $3.558 E-04$ & $3.981 E-05$ & $3.593 E+00$ \\
\hline \multicolumn{9}{|l|}{ Aluminum Hydrogen EDTA } \\
\hline \multicolumn{9}{|l|}{ Aluminum Hydroxide } \\
\hline Benzene & $9.395 E-03$ & & $3.775 E+00$ & $3.743 E+00$ & $3.148 E-02$ & $3.743 E+00$ & $2.516 E-05$ & $4.503 E-01$ \\
\hline Bis(2-ethylhexyl)phthalate (BEHP) & $3.264 E-04$ & & \begin{tabular}{|l|}
$1.127 E-05$ \\
\end{tabular} & \begin{tabular}{|l|}
$1.264 E-07$ \\
\end{tabular} & 1.114E-05 & $1.051 E-07$ & $2.133 E-08$ & $1.791 E+00$ \\
\hline \multicolumn{9}{|l|}{ Benzo(a)pyrene (BaP) } \\
\hline Phenol & $9.769 E-06$ & & $6.855 E-07$ & $1.396 E-08$ & $6.715 E-07$ & $1.351 E-08$ & $4.469 E-10$ & $6.495 E-03$ \\
\hline \multicolumn{9}{|l|}{ Calcium Chloride Oxide } \\
\hline \multicolumn{9}{|l|}{ Calcium Oxalate } \\
\hline \multicolumn{9}{|l|}{ Calcium Oxalate Monohydrate } \\
\hline \multicolumn{9}{|l|}{ Calcium Carbonate } \\
\hline \multicolumn{9}{|l|}{ Calcium Dichromate (VI) } \\
\hline \multicolumn{9}{|l|}{ Calcium Fluoride } \\
\hline Pentachlorophenol & $5.681 E-03$ & & $4.004 E-04$ & $8.221 E-06$ & $3.922 E-04$ & $7.845 E-06$ & $3.753 E-07$ & $3.814 E+00$ \\
\hline \multicolumn{9}{|l|}{ Hexachlorobenzene } \\
\hline Chlorobenzene & $1.668 \mathrm{E}-02$ & & $3.444 E+00$ & $3.389 E+00$ & $5.545 E-02$ & $3.389 E+00$ & 4.472E-05 & $8.015 E-01$ \\
\hline 2-Chloronaphthalene & $4.514 E-02$ & & $2.087 E+00$ & $1.945 E+00$ & $1.420 E-01$ & $1.945 E+00$ & 2.217E-04 & $2.249 E+00$ \\
\hline Carbon Dioxide & $4.034 \mathrm{E}-03$ & & $4.124 \mathrm{E}+00$ & $4.113 \mathrm{E}+00$ & 1.117E-02 & $4.113 \mathrm{E}+00$ & $8.645 \mathrm{E}-06$ & $3.457 \mathrm{E}-02$ \\
\hline \multicolumn{9}{|l|}{ Cesium Acetate } \\
\hline Dibenz[a,h]anthracene & $7.568 E-09$ & & & & & & & $9.406 E-03$ \\
\hline 1,2-dibromoethane & $2.961 E-01$ & & $1.462 E+01$ & $1.368 E+01$ & $9.366 \mathrm{E}-01$ & $1.368 E+01$ & $7.478 E-04$ & $1.437 E+01$ \\
\hline Diethyl phthalate & $1.538 E-03$ & & \begin{tabular}{|l|}
$3.211 E-05$ \\
\end{tabular} & \begin{tabular}{|l|}
$1.984 E-07$ \\
\end{tabular} & $3.191 E-05$ & \begin{tabular}{|l|}
$1.829 E-07$ \\
\end{tabular} & $1.554 E-08$ & $3.284 E+00$ \\
\hline \multicolumn{9}{|l|}{ Iron (III) Chloride } \\
\hline \multicolumn{9}{|l|}{ Iron (III) Oxalate } \\
\hline \multicolumn{9}{|l|}{ Iron (III) Hydrogen EDTA } \\
\hline Iron (III) Hydroxide & & & & & & & & \\
\hline Formic Acid, dimer & & & & & & & & \\
\hline Hydrofluoride, dimer & & & & & & & & \\
\hline 2,2'-Iminobisacetic acid & & & & & & & & \\
\hline Water & $4.870 \mathrm{E}+04$ & $4.536 \mathrm{E}+04$ & $4.793 \mathrm{E}+04$ & $7.369 \mathrm{E}+02$ & $4.720 \mathrm{E}+04$ & $7.071 \mathrm{E}+02$ & $2.982 \mathrm{E}+01$ & $4.927 \mathrm{E}+06$ \\
\hline Sulfuric Acid & & & & & & & & \\
\hline Hydrogen Chloride & & & & & & & & $6.020 \mathrm{E}-08$ \\
\hline Formic Acid & & & & & & & & $2.927 \mathrm{E}-07$ \\
\hline Hydrofluoric Acid & & & & & & & & $1.686 \mathrm{E}-07$ \\
\hline Mercury (II) Chloride & & & & & & & & \\
\hline Mercury (elemental) & $4.378 \mathrm{E}+00$ & & $3.408 \mathrm{E}+00$ & $9.732 \mathrm{E}-01$ & $2.435 \mathrm{E}+00$ & 7.715E-01 & $2.016 \mathrm{E}-01$ & $6.271 \mathrm{E}+01$ \\
\hline Mercury (II) Oxide & & & & & & & & $1.787 \mathrm{E}-07$ \\
\hline Nitrous (III) Acid & $5.266 \mathrm{E}-07$ & & 4.936E-09 & $2.498 \mathrm{E}-11$ & 4.911E-09 & & & $8.501 \mathrm{E}-03$ \\
\hline Nitric Acid & & & & & & & & \\
\hline Hexachlorobutadiene & $5.495 E-04$ & & $4.169 E+00$ & $4.167 E+00$ & $1.850 E-03$ & $4.167 E+00$ & $6.590 E-06$ & $3.245 E-02$ \\
\hline Potassium Citrate & & & & & & & & \\
\hline Potassium Chloride & & & & & & & & \\
\hline Potassium Glycolate & & & & & & & & \\
\hline Potassium Nitrate $(\mathrm{VI})$ & & & & & & & & \\
\hline gamma-BHC (Lindane) & $6.275 E-02$ & & $1.866 E-01$ & $8.759 E-02$ & $9.905 E-02$ & $8.739 \mathrm{E}-02$ & $1.951 E-04$ & $4.103 E+00$ \\
\hline 4-methyl-2-pentanone (MIBK) & $6.870 \mathrm{E}-02$ & & $9.121 E-01$ & \begin{tabular}{|l|}
$7.271 E-01$ \\
\end{tabular} & $1.850 \mathrm{E}-01$ & $7.269 \mathrm{E}-01$ & $1.471 E-04$ & $3.467 E+00$ \\
\hline Magnesium Oxalate & & & & & & & & \\
\hline Magnesium Dichromate $(\mathrm{VI})$ & & & & & & & & \\
\hline
\end{tabular}


WSRC-IR-2002-00550, Kev. I SRT-RPP-2002-0027.

\begin{tabular}{|c|c|c|c|c|c|c|c|c|}
\hline Stream & $\begin{array}{c}\text { IC } \\
\text { Condensate }\end{array}$ & $\begin{array}{l}\text { Super } \\
\text { Steam } 2\end{array}$ & \begin{tabular}{|c|} 
Stm Eject \\
2 Out
\end{tabular} & AC Vapor & $\begin{array}{c}\mathrm{AC} \\
\text { Condensate }\end{array}$ & $\begin{array}{l}\text { Demist } \\
\text { Vapor }\end{array}$ & $\begin{array}{l}\text { Demist } \\
\text { Liquid }\end{array}$ & $\begin{array}{l}\text { Evap Cond } \\
\text { Tk Out }\end{array}$ \\
\hline \multicolumn{9}{|l|}{ Magnesium Hydroxide } \\
\hline \multicolumn{9}{|l|}{ Manganese (II) Oxalate } \\
\hline Nitrogen & $1.220 \mathrm{E}-01$ & & $8.908 \mathrm{E}+03$ & $8.907 E+03$ & $4.142 \mathrm{E}-01$ & $8.907 \mathrm{E}+03$ & $3.302 \mathrm{E}-04$ & $2.302 \mathrm{E}+00$ \\
\hline \multicolumn{9}{|l|}{ Sodium Oxalate } \\
\hline \multicolumn{9}{|l|}{ Sodium Glycolate } \\
\hline \multicolumn{9}{|l|}{ Sodium Nitrite } \\
\hline \multicolumn{9}{|l|}{ Sodium Hydroxide } \\
\hline Naphthalene & $3.753 E-02$ & & $2.480 E+00$ & $2.360 E+00$ & $1.204 E-01$ & $2.360 E+00$ & $1.139 E-04$ & $1.828 E+00$ \\
\hline \multicolumn{9}{|l|}{ Sodium Aluminosilicate Gel } \\
\hline \multicolumn{9}{|l|}{ Nickel (II) Oxalate } \\
\hline \multicolumn{9}{|l|}{ Silicon Dioxide } \\
\hline \multicolumn{9}{|l|}{ Sulfur Trioxide } \\
\hline 1,2,4-trichlorobenzene & 1.587E-02 & & $3.466 E+00$ & $3.413 E+00$ & $5.272 E-02$ & $3.413 E+00$ & $6.842 E-05$ & 7.797E-01 \\
\hline 1,2,3-trichloropropane & $5.197 E-02$ & & $1.819 E+00$ & $1.659 E+00$ & $1.600 E-01$ & $1.659 E+00$ & 1.283E-04 & $2.539 E+00$ \\
\hline TOLUENE & $8.303 E-03$ & & $3.823 E+00$ & $3.796 E+00$ & $2.782 E-02$ & $3.796 E+00$ & 2.245E-05 & $3.981 E-01$ \\
\hline \multicolumn{9}{|l|}{ Zinc Dichloride Pentoxide } \\
\hline \multicolumn{9}{|l|}{ Zinc Oxalate } \\
\hline \multicolumn{9}{|l|}{ Zinc Hydroxide } \\
\hline \multicolumn{9}{|l|}{ Zirconium EDTA } \\
\hline \multicolumn{9}{|l|}{ Zirconium Oxide } \\
\hline Total g/hr & $4.870 \mathrm{E}+04$ & $4.536 \mathrm{E}+04$ & $5.959 \mathrm{E}+04$ & $1.239 E+04$ & $4.720 \mathrm{E}+04$ & $1.236 \mathrm{E}+04$ & $3.003 \mathrm{E}+01$ & $4.927 \mathrm{E}+06$ \\
\hline \multicolumn{9}{|l|}{ Redox Pot, volts } \\
\hline E-Con, cm2/ohm mol & $4.621 \mathrm{E}-05$ & & 6.945E-05 & & $8.127 \mathrm{E}-05$ & & & $6.196 \mathrm{E}-01$ \\
\hline Abs Visc, cP & $6.536 \mathrm{E}-01$ & $2.077 \mathrm{E}-01$ & 3.692E-01 & & $6.536 \mathrm{E}-01$ & & & $8.907 \mathrm{E}-01$ \\
\hline Rel Visc & $1.000 \mathrm{E}+00$ & $1.000 \mathrm{E}+00$ & $1.000 \mathrm{E}+00$ & & $1.000 \mathrm{E}+00$ & & & $1.000 \mathrm{E}+00$ \\
\hline Ionic Strength & $7.863 \mathrm{E}-07$ & $1.250 \mathrm{E}-06$ & 9.357E-07 & & $1.444 \mathrm{E}-06$ & $1.620 \mathrm{E}-06$ & $1.620 \mathrm{E}-06$ & $1.988 \mathrm{E}-07$ \\
\hline
\end{tabular}




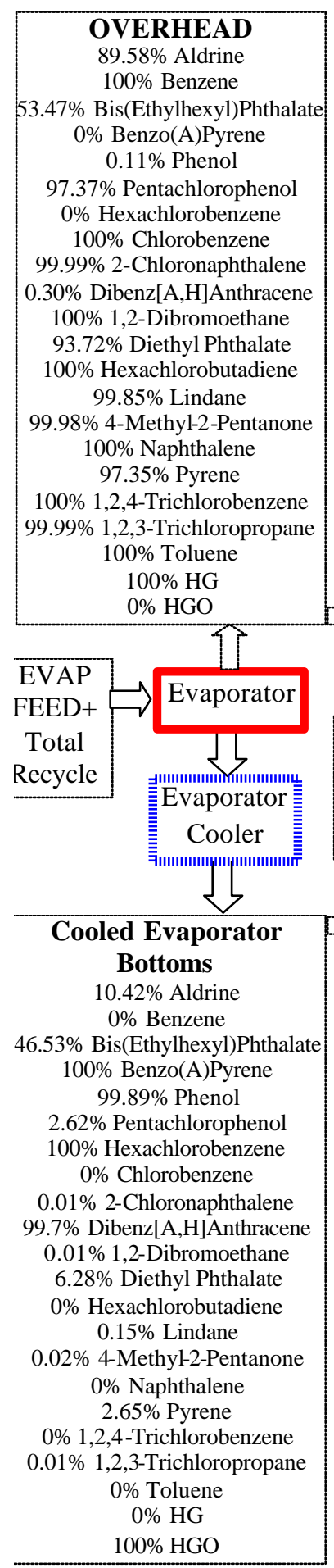

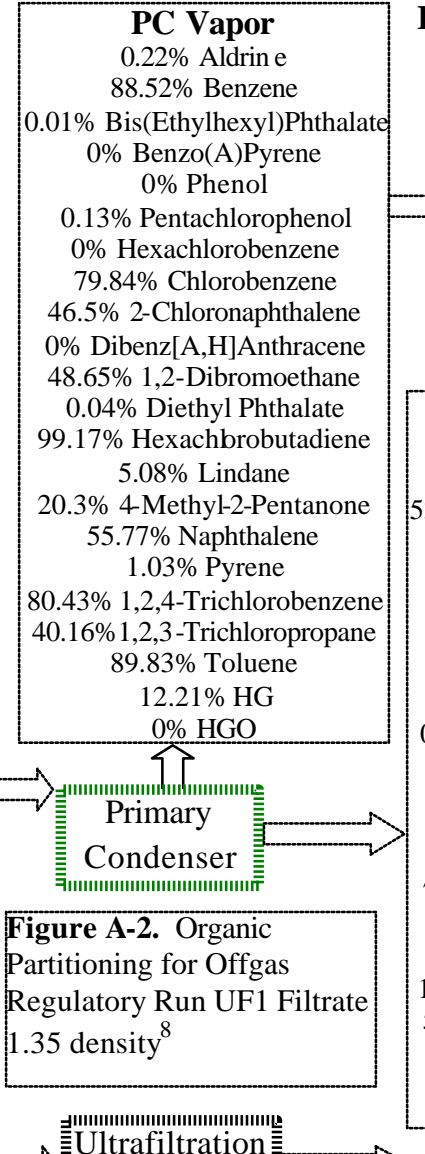

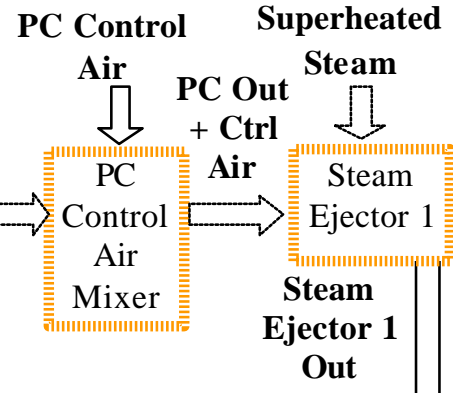

\section{PC Condensate} $11.47 \%$ Benzene

3.46\% Bis(Ethylhexyl)Phthalate 0\% Benzo(A)Pyrene $0.11 \%$ Phenol

97.24\% Pentachlorophenol $0 \%$ Hexachlorobenzene 20.16\% Chlorobenzene 53.49\%2-Chloronaphthalene 0.30\% Dibenz[A,H]Anthracene $51.34 \%$ 1,2-Dibromoethane 93.69\% Diethyl Phthalate $0.83 \%$ Hexachlorobutadiene $94.77 \%$ Lindane

79.68\% 4-Methyl-2-Pentanone 44.23\% Naphthalene 96.31\% Pyrene

$19.57 \%$ 1,2,4-Trichlorobenzene $59.83 \% 1,2,3$-Trichloropropane $10.17 \%$ Toluene $87.79 \%$ HG 竐Ultrafiltration

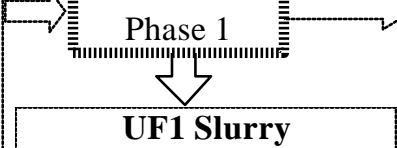

UF1 Slurry $0.80 \%$ Aldrine $3.55 \%$ Bis(Ethylhexyl)Phthalate 99.99\% Benzo(A)Pyrene 7.62\% Phenol

$0.20 \%$ Pentachlorophenol 99.97\% Hexachlorobenzene 0\% Chlorobenzene

0\% 2-Chloronaphthalene

7.61\% Dibenz[A,H]Anthracene 0\% 1,2-Dibromoethane $0.48 \%$ Diethyl Phthalate $0 \%$ Hexachlorobutadien $0.01 \%$ Lindane

0\% 4-Methyl2-Pentanone $0 \%$ Naphthalene

$0.20 \%$ Pyrene

$0 \%$ 1,2,4-Trichlorobenzene 0\%1,2,3-Trichloropropane $0 \%$ Toluene $0 \% \mathrm{HG}$

\section{$0 \%$ HGO}

\begin{tabular}{|c|}
\hline UF1 Filtrate \\
$9.63 \%$ Aldrine \\
$0 \%$ Benzene \\
42.98\% Bis(Ethylhexyl)Phthalate \\
$0.01 \%$ Benzo(A)Pyrene \\
$92.27 \%$ Phenol \\
$2.42 \%$ Pentachlorophenol \\
$0.03 \%$ Hexachlorobenzene \\
$0 \%$ Chlorobenzene \\
$0.01 \% 2$-Chloronaphthalene \\
92.09\% Dibenz[A,H]Anthracene \\
$0 \% 1,2$-Dibromoethane \\
$5.80 \%$ Diethyl Phthalate \\
$0 \%$ Hexachlorobutadiene \\
$0.14 \%$ Lindane \\
$0.01 \%$-Methyl-2-Pentanone \\
$0 \%$ Naphthalene \\
$2.45 \%$ Pyrene \\
$0 \% 1,2,4-$ Trichlorobenzene \\
$0.01 \% 1,2,3-$ Trichloropropane \\
$0 \%$ Toluene \\
$0 \%$ Hg \\
26.08\% HgO \\
\hline
\end{tabular}

IC Vapor

$0.08 \%$ Aldrine

88.3\% Benzene

$0 \%$ Bis(Ethylhexyl)Phthalate

$0 \%$ Phenol

$0.01 \%$ Pentachlorophenol

$0 \%$ Hexachlorobenzene

$79.46 \%$ Chlorobenzene

$45.52 \%$ 2-Chloronaphthalene

$0 \%$ Dibenz[A,H]Anthracene

47.69\% 1,2-Dibromoethane

0\% Diethyl Phthalate

99.16\% Hexachlorobutadiene $3.81 \%$ Lindane

18.88\% 4-Methyl-2-Pentanone $54.94 \%$ Naphthalene $0.56 \%$ Pyrene

$80.06 \%$ 1,2,4-Trichlorobenzene $39.05 \% 1,2,3$-Trichloropropane $89.64 \%$ Toluene $5.35 \% \mathrm{Hg}$ $0 \% \mathrm{HgO}$

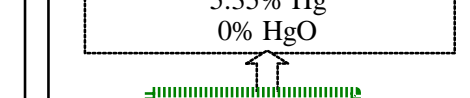

N)

Inter

Condenser

$$
\zeta^{1}
$$

\section{IC Condensate}

$0.14 \%$ Aldrine

$0.22 \%$ Benzene

$0.01 \%$ Bis(Ethylhexyl)Phthalate

0\% Benzo(A)Pyrene

$$
0 \% \text { Phenol }
$$

$0.12 \%$ Pentachlorophenol

$0 \%$ Hexachlorobenzene

$0.38 \%$ Chlorobenzene

$0.98 \%$ 2-Chloronaphthalene

0\% Dibenz[A,H]Anthracene

$0.97 \%$ 1,2-Dibromoethane

$0.04 \%$ Diethyl Phthalate

$0.01 \%$ Hexachlorobutadiene $1.28 \%$ Lindane

1.42\% 4-Methyl-2-Pentanone

$0.83 \%$ Naphthalene

$0.47 \%$ Pyrene

$0.37 \%$ 1,2,4Trichlorobenzene

1.12\%1,2,3-Trichloropropane

$0.19 \%$ Toluene

$6.87 \% \mathrm{Hg}$

$0 \% \mathrm{HgO}$
0\% Benzo(A)Pyrene

Superheated

Steam

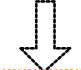

Steam

Steam

Ejector 2

Out

t

$\mid$
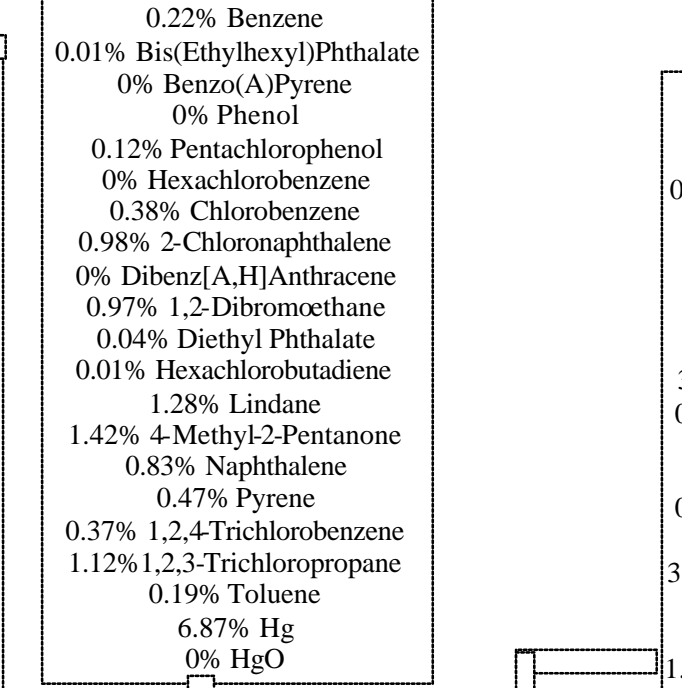$$
0
$$

(Ethylhexyl)Pht

$0.01 \%$ Pentachlorophenol

0\% Hexachlorobenzene

$1.28 \%$ Chlorobenzene

3.09\% 2-Chloronaphthalene

0\% Dibenz[A,H]Anthracene

3.06\% 1,2-Dibromoethane

0\% Diethyl Phthalate

0.04\% Hexachlorobutadiene $2.02 \%$ Lindane

3.83\% 4-Methyl-2-Pentanone

$2.67 \%$ Naphthalen

$0.42 \%$ Pyrene

$1.22 \%$ 1,2,4-Trichlorobenzene

$3.43 \%$ 1,2,3-Trichloropropane

$0.65 \%$ Toluene

$3.82 \% \mathrm{Hg}$

$0 \% \mathrm{HgO}$
2002-00550, Kev. 1

SRT-RPP-2002-0027

Demist Vapor

$0.01 \%$ Aldrine

87.57\% Benzene

0\% Bis(Ethylhexyl)Phthalat

$$
0 \% \text { Phenol }
$$

$0 \%$ Pentachlorophenol

0\% Hexachlorobenzene

78.18\% Chlo robenzene

42.42\% 2-Chloronaphthalen

$0 \%$ Dibenz $[\mathrm{A}, \mathrm{H}]$ Anthracene

44.63\% 1,2-Dibromoethane

$0 \%$ Diethyl Phthalate

99.12\% Hexachlorobutadien

$1.78 \%$ Lindane

$15.05 \%$ 4-Methyl-2-Pentanon

$52.27 \%$ Naphthalene

$0.13 \%$ Pyrene

78.84\% 1,2,4-Trichlorobenze $35.61 \%$ 1,2,3-Trichloropropar

$88.99 \%$ Toluene

$1.21 \% \mathrm{Hg}$

$0 \% \mathrm{HgO}$

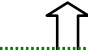

AC Demister

ำเ

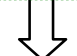

Demist liquid

$0 \%$ Aldrine

0\% Benzene

$0 \%$ Bis(Ethylhexyl)Phthalate

0\% Benzo(A)Pyrene

$0 \%$ Phenol

$0 \%$ Pentachlorophenol

$0 \%$ Hexachlorobenzene

$0 \%$ Chlorobenzene

$0 \% 2$-Chloronaphthalene

0\% Dibenz[A,H]Anthracene

$0 \%$ 1,2-Dibromoethane

$0 \%$ Hexachlorobutadiene

$0 \%$ Lindane

0\% 4-Methyl2-Pentanone

$0 \%$ Naphthalene

$0.01 \%$ Pyrene

$0 \%$ 1,2,4-Trichlorobenzene

0\%1,2,3-Trichloropropane

$0 \%$ Toluene

$0.32 \% \mathrm{Hg}$

$0 \% \mathrm{HgO}$ 
WSKC-'IR-2UUZ-0U550, Rev. I

SRT-RPP-2002-0027.

Table A- 2. Waste Feed Evaporator Offgas Model Output for UF1 Filtrate Density of $1.35 \mathrm{~g} / \mathrm{ml}^{8}$

\begin{tabular}{|c|c|c|c|c|c|c|c|c|c|}
\hline Stream & $\begin{array}{l}\text { HLW OG } \\
\text { Cond }\end{array}$ & $\begin{array}{c}\text { Total } \\
\text { Recycle }\end{array}$ & Evap Feed & $\begin{array}{l}\text { EVAP } \\
\text { REDRXN } \\
\text { OUT }\end{array}$ & $\begin{array}{c}\text { Air } \\
\text { Inleakage }\end{array}$ & $\begin{array}{c}\text { Evap } \\
\text { Contents }\end{array}$ & Overhead & $\begin{array}{c}\text { Evap } \\
\text { Bottoms }\end{array}$ & $\begin{array}{c}\text { Cooled } \\
\text { Evap Bott }\end{array}$ \\
\hline Phase & Mixed & Mixed & Mixed & Mixed & Vapor & Mixed & Vapor & Mixed & Mixed \\
\hline Temperature, C & 25 & 25 & 25 & 50 & 25 & 50 & 50 & 50 & 25 \\
\hline Pressure, atm & 1 & 1 & 1 & 0.108335 & 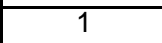 & 0.102137 & 0.102137 & 0.102137 & 1 \\
\hline $\mathrm{pH}$ & 11.2694 & 12.8607 & 13.0705 & 12.6386 & & 13.1314 & & 13.1314 & 14.034 \\
\hline Total $\mathrm{mol} / \mathrm{hr}$ & 188132 & 7912.79 & 197463 & 393540 & 135.18 & 393679 & 321648 & 72031.1 & 71852.3 \\
\hline Flow Units & $\mathrm{g} / \mathrm{hr}$ & $\mathrm{g} / \mathrm{hr}$ & $\mathrm{g} / \mathrm{hr}$ & $\mathrm{g} / \mathrm{hr}$ & $\mathrm{g} / \mathrm{hr}$ & $\mathrm{g} / \mathrm{hr}$ & $\mathrm{g} / \mathrm{hr}$ & $\mathrm{g} / \mathrm{hr}$ & $\mathrm{g} / \mathrm{hr}$ \\
\hline Acetic Acid, Dimer & & & & $1.584 \mathrm{E}-18$ & & 1.194E-18 & $1.194 \mathrm{E}-18$ & & \\
\hline Acetic Acid & & & & $2.804 \mathrm{E}-06$ & & $2.715 \mathrm{E}-06$ & $2.715 \mathrm{E}-06$ & & \\
\hline Aluminum Oxide & $1.231 \mathrm{E}+00$ & $6.382 \mathrm{E}+01$ & $2.657 \mathrm{E}+03$ & $4.704 \mathrm{E}+03$ & & $4.740 \mathrm{E}+03$ & & $4.740 \mathrm{E}+03$ & $4.737 \mathrm{E}+03$ \\
\hline Aldrin & & $7.204 E-05$ & \begin{tabular}{|l|}
$4.195 E+00$ \\
\end{tabular} & \begin{tabular}{|l|}
$4.195 E+00$ \\
\end{tabular} & & $4.195 E+00$ & $3.758 E+00$ & $4.371 E-01$ & $4.371 E-01$ \\
\hline Aluminum Hydrogen EDTA & & $1.727 \mathrm{E}+01$ & $1.741 \mathrm{E}+03$ & $1.209 \mathrm{E}+03$ & & $1.208 \mathrm{E}+03$ & & $1.208 \mathrm{E}+03$ & $1.188 \mathrm{E}+03$ \\
\hline Aluminum Hydroxide & & $4.775 \mathrm{E}+01$ & $3.103 \mathrm{E}+03$ & & & & & & \\
\hline Benzene & & \begin{tabular}{|l|}
$3.537 E-07$ \\
\end{tabular} & $4.194 E+00$ & $4.194 E+00$ & & $4.194 E+00$ & $4.194 E+00$ & $2.807 E-05$ & 2.807E-05 \\
\hline Bis(2-ethylhexyl)phthalate (BEHP) & & $9.539 E-04$ & $4.193 E+00$ & $4.194 E+00$ & & $4.194 E+00$ & $2.243 E+00$ & $1.952 E+00$ & $1.952 E+00$ \\
\hline Benzo(a)pyrene (BaP) & & 6.746E-06 & $4.181 E+00$ & $4.181 E+00$ & & $4.181 E+00$ & & $4.181 E+00$ & $4.181 E+00$ \\
\hline Phenol & & $5.364 E-02$ & $4.196 E+00$ & $4.249 E+00$ & & $4.249 E+00$ & $4.512 E-03$ & $4.245 E+00$ & $4.245 E+00$ \\
\hline Calcium Chloride Oxide & $5.455 \mathrm{E}+02$ & & & & & & & & \\
\hline Calcium Oxalate & $6.886 \mathrm{E}+00$ & & $6.736 \mathrm{E}+02$ & $2.141 \mathrm{E}+02$ & & $3.096 \mathrm{E}+02$ & & $3.096 \mathrm{E}+02$ & $6.266 \mathrm{E}+0$ \\
\hline Calcium Oxalate Monohydrate & $2.059 \mathrm{E}+00$ & & & & & & & & \\
\hline Calcium Carbonate & & $5.280 \mathrm{E}+00$ & & & & & & & \\
\hline Calcium Citrate & & $1.867 \mathrm{E}+00$ & & & & & & & \\
\hline Calcium Fluoride & $5.358 \mathrm{E}+02$ & & $3.237 \mathrm{E}+02$ & $1.660 \mathrm{E}+03$ & & $1.601 \mathrm{E}+03$ & & $1.601 \mathrm{E}+03$ & $1.408 \mathrm{E}+03$ \\
\hline Pentachlorophenol & & $1.161 E-03$ & $4.196 E+00$ & \begin{tabular}{|l|}
$4.197 E+00$ \\
\end{tabular} & & $4.197 E+00$ & $4.087 E+00$ & 1.102E-01 & $1.102 E-01$ \\
\hline Hexachlorobenzene & & $1.610 E-05$ & \begin{tabular}{|l|}
$4.188 E+00$ \\
\end{tabular} & $4.188 E+00$ & & $4.188 E+00$ & & $4.188 E+00$ & $4.188 E+00$ \\
\hline Chlorob & & $7.586 E-07$ & $4.190 E+00$ & $4.190 E+00$ & & $4.190 E+00$ & $4.190 E+00$ & $6.047 E-05$ & $6.047 E-05$ \\
\hline 2-Chlorona & & \begin{tabular}{|l|}
$2.038 E-06$ \\
\end{tabular} & \begin{tabular}{|l|}
$4.194 E+00$ \\
\end{tabular} & $4.194 E+00$ & & $4.194 E+00$ & \begin{tabular}{|l|}
$4.194 E+00$ \\
\end{tabular} & $2.439 E-04$ & $2.439 E-04$ \\
\hline Carbon Dioxide & & $1.002 \mathrm{E}+03$ & $7.847 \mathrm{E}+04$ & $7.948 \mathrm{E}+04$ & $49 \mathrm{E}+00$ & $7.948 \mathrm{E}+04$ & \begin{tabular}{|l|}
$5.045 \mathrm{E}-02$ \\
\end{tabular} & $7.948 \mathrm{E}+04$ & $7.948 \mathrm{E}+04$ \\
\hline Cesium Acetate & & $2.524 \mathrm{E}+00$ & $1.972 \mathrm{E}+02$ & \begin{tabular}{|l|}
$1.998 \mathrm{E}+02$ \\
\end{tabular} & & $1.998 \mathrm{E}+02$ & & $1.998 \mathrm{E}+02$ & $1.998 \mathrm{E}+02$ \\
\hline Dibenz[a,h]anthracene & & \begin{tabular}{|l|}
$5.199 E-04$ \\
\end{tabular} & $4.191 E+00$ & $4.191 E+00$ & & $4.191 E+00$ & $1.244 E-02$ & $4.179 E+00$ & $4.179 E+00$ \\
\hline 1,2-dibromoethane & & $1.823 E-05$ & $2.806 E+01$ & $2.806 E+01$ & & $2.806 E+01$ & $2.806 E+01$ & $1.446 E-03$ & $1.446 E-03$ \\
\hline Diethyl phthalate & & $3.287 E-03$ & $4.202 E+00$ & $4.205 E+00$ & & $4.205 E+00$ & $3.941 E+00$ & $2.639 E-01$ & $2.639 \mathrm{E}-01$ \\
\hline Iron (III) Chloride & $1.159 \mathrm{E}+00$ & & & & & & & & \\
\hline Iron (III) Hydrogen EDTA & & $4.792 \mathrm{E}+00$ & & $5.994 \mathrm{E}+02$ & & $5.994 \mathrm{E}+02$ & & $5.994 \mathrm{E}+02$ & $5.994 \mathrm{E}+02$ \\
\hline Iron (III) Hydroxide & $1.547 \mathrm{E}+02$ & $2.866 \mathrm{E}+01$ & & & & & & & \\
\hline Formic Acid, dimer & & & & $7.829 \mathrm{E}-25$ & & $6.209 \mathrm{E}-25$ & $6.209 \mathrm{E}-25$ & & \\
\hline Hydrofluoride, dimer & & & & \begin{tabular}{|l|}
$2.203 \mathrm{E}-22$ \\
\end{tabular} & & $1.184 \mathrm{E}-22$ & $1.184 \mathrm{E}-22$ & & \\
\hline 2,2'-Iminobisacetic acid & & $1.449 \mathrm{E}+01$ & $1.132 \mathrm{E}+03$ & $1.147 \mathrm{E}+03$ & & $1.147 \mathrm{E}+03$ & & $1.147 \mathrm{E}+03$ & $1.147 \mathrm{E}+03$ \\
\hline Water & $3.361 \mathrm{E}+06$ & $1.371 \mathrm{E}+05$ & $3.290 \mathrm{E}+06$ & 6.789E+06 & $1.819 \mathrm{E}+01$ & $6.790 \mathrm{E}+06$ & $5.792 E+06$ & $9.977 \mathrm{E}+05$ & $1.001 \mathrm{E}+06$ \\
\hline \multicolumn{10}{|l|}{ Sulfuric Acid } \\
\hline Hydrogen Chloride & $1.862 \mathrm{E}+04$ & $1.301 \mathrm{E}+02$ & & $1.041 \mathrm{E}+04$ & & $1.041 \mathrm{E}+04$ & $4.263 \mathrm{E}-11$ & $1.041 \mathrm{E}+04$ & $1.041 \mathrm{E}+04$ \\
\hline Formic Acid & & $5.069 \mathrm{E}+00$ & $3.961 \mathrm{E}+02$ & $4.011 \mathrm{E}+02$ & & $4.011 \mathrm{E}+02$ & 3.091E-07 & $4.011 \mathrm{E}+02$ & $4.011 \mathrm{E}+02$ \\
\hline Hydrofluoric Acid & $2.116 \mathrm{E}+01$ & $2.471 \mathrm{E}+01$ & $3.388 \mathrm{E}+03$ & $3.023 \mathrm{E}+03$ & & $3.053 \mathrm{E}+03$ & 1.450E-07 & $3.053 \mathrm{E}+03$ & $1.345 \mathrm{E}+03$ \\
\hline Mercury (II) Chloride & & & & \begin{tabular}{|l|}
$2.442 \mathrm{E}-07$ \\
\end{tabular} & & $1.603 \mathrm{E}-07$ & \begin{tabular}{|l}
$1.603 \mathrm{E}-07$ \\
\end{tabular} & & \\
\hline Mercury (elemental) & & 1.744E-06 & & $6.374 \mathrm{E}+01$ & & $6.374 \mathrm{E}+01$ & $6.374 \mathrm{E}+01$ & $1.380 \mathrm{E}-04$ & $1.380 \mathrm{E}-04$ \\
\hline Mercury (II) Oxide & & $1.534 \mathrm{E}+00$ & $1.361 \mathrm{E}+02$ & \begin{tabular}{|l|}
$6.878 \mathrm{E}+01$ \\
\end{tabular} & & $6.878 \mathrm{E}+01$ & & $6.878 \mathrm{E}+01$ & $6.878 \mathrm{E}+01$ \\
\hline Nitrous (III) Acid & $3.708 \mathrm{E}+03$ & $4.828 \mathrm{E}+02$ & $9.353 \mathrm{E}+04$ & $9.653 \mathrm{E}+04$ & & $9.378 \mathrm{E}+04$ & 6.469E-03 & $9.378 \mathrm{E}+04$ & $9.331 \mathrm{E}+04$ \\
\hline Nitric Acid & $1.888 \mathrm{E}+03$ & $4.675 \mathrm{E}+03$ & $4.511 \mathrm{E}+04$ & $6.653 \mathrm{E}+04$ & & $6.653 \mathrm{E}+04$ & \begin{tabular}{|l|}
$3.522 \mathrm{E}-12$ \\
\end{tabular} & $6.653 \mathrm{E}+04$ & $6.653 \mathrm{E}+04$ \\
\hline Hexachlorobutadien & & $1.590 \mathrm{E}-08$ & $4.200 E+00$ & $4.200 E+00$ & & $4.200 E+00$ & $4.200 E+00$ & 5.547E-06 & 5.547E-06 \\
\hline Potassium Citrate & & $3.384 \mathrm{E}+01$ & $2.823 \mathrm{E}+03$ & $2.859 \mathrm{E}+03$ & & $2.859 \mathrm{E}+03$ & & $2.859 \mathrm{E}+03$ & $2.859 \mathrm{E}+03$ \\
\hline Potassium Chloride & $1.845 \mathrm{E}+04$ & $4.797 \mathrm{E}+02$ & $1.350 \mathrm{E}+03$ & \begin{tabular}{|l|}
$3.783 \mathrm{E}+04$ \\
\end{tabular} & & $3.783 \mathrm{E}+04$ & & $3.783 \mathrm{E}+04$ & $3.783 \mathrm{E}+04$ \\
\hline Potassium Nitrate (VI) & & & $2.380 \mathrm{E}+04$ & & & & & & \\
\hline gamma-BHC (Lindane) & & $4.442 E-05$ & \begin{tabular}{|l|}
$4.209 E+00$ \\
\end{tabular} & +00 & & $4.209 E+00$ & $4.203 E+00$ & EE-03 & $6.155 E-03$ \\
\hline methyl-2-pentanone (MI) & & 8.460E-06 & 4.197E+00 & 4.197E+00 & & 4.197E+00 & $4.196 E+00$ & $6.698 E-04$ & $6.698 E-04$ \\
\hline Magnesium Oxalate & $2.060 \mathrm{E}+00$ & $1.488 \mathrm{E}-03$ & & \begin{tabular}{|l|}
$2.956 \mathrm{E}-02$ \\
\end{tabular} & & $1.704 \mathrm{E}-02$ & & $1.704 \mathrm{E}-02$ & $2.043 \mathrm{E}-03$ \\
\hline Magnesium Hydroxide & $2.368 \mathrm{E}+02$ & $1.301 \mathrm{E}+00$ & & $2.391 \mathrm{E}+02$ & & $2.391 \mathrm{E}+02$ & & $2.391 \mathrm{E}+02$ & $2.392 \mathrm{E}+02$ \\
\hline Manganese (II) Oxalate & & \begin{tabular}{|l|}
$2.485 \mathrm{E}-01$ \\
\end{tabular} & & $2.679 \mathrm{E}+00$ & & $2.679 \mathrm{E}+00$ & & $2.679 \mathrm{E}+00$ & $2.679 \mathrm{E}+00$ \\
\hline Manganese (II) Hydroxide & & $1.512 \mathrm{E}+00$ & & & & & & & \\
\hline
\end{tabular}


WSKC'-'IR-2UU2-00550, Kev. I

SRT-RPP-2002-0027.

\begin{tabular}{|c|c|c|c|c|c|c|c|c|c|}
\hline Stream & $\begin{array}{l}\text { HLW OG } \\
\text { Cond }\end{array}$ & $\begin{array}{c}\text { Total } \\
\text { Recycle }\end{array}$ & Evap Feed & $\begin{array}{l}\text { EVAP } \\
\text { REDRXN } \\
\text { OUT }\end{array}$ & $\begin{array}{c}\text { Air } \\
\text { Inleakage }\end{array}$ & $\begin{array}{c}\text { Evap } \\
\text { Contents }\end{array}$ & Overhead & $\begin{array}{c}\text { Evap } \\
\text { Bottoms }\end{array}$ & $\begin{array}{c}\text { Cooled } \\
\text { Evap Bott }\end{array}$ \\
\hline Nitrogen & & $1.743 \mathrm{E}-06$ & & $1.743 \mathrm{E}-06$ & $2.970 \mathrm{E}+03$ & $2.970 \mathrm{E}+03$ & $2.970 \mathrm{E}+03$ & $1.379 \mathrm{E}-04$ & $1.379 \mathrm{E}-04$ \\
\hline Sodium Oxalate & & & $1.433 E+04$ & $1.382 \mathrm{E}+04$ & & $1.372 \mathrm{E}+04$ & & $1.372 \mathrm{E}+04$ & $1.339 \mathrm{E}+04$ \\
\hline Sodium Chromate (VI) & & $1.069 \mathrm{E}+02$ & $8.349 \mathrm{E}+03$ & $8.456 \mathrm{E}+03$ & & $8.456 \mathrm{E}+03$ & & $8.456 \mathrm{E}+03$ & $8.456 \mathrm{E}+03$ \\
\hline Sodium Floride Sulfate & & & & & & & & & $1.662 \mathrm{E}+04$ \\
\hline Sodium Acetate & & $2.349 E+00$ & $1.835 \mathrm{E}+02$ & $1.859 \mathrm{E}+02$ & & $1.859 \mathrm{E}+02$ & & $1.859 \mathrm{E}+02$ & $1.859 \mathrm{E}+02$ \\
\hline Sodium Glycolate & & $5.832 \mathrm{E}+01$ & $4.556 \mathrm{E}+03$ & $4.615 \mathrm{E}+03$ & & $4.615 \mathrm{E}+03$ & & $4.615 \mathrm{E}+03$ & $4.615 \mathrm{E}+03$ \\
\hline Sodium Nitrite & $3.054 \mathrm{E}+03$ & $1.966 \mathrm{E}+03$ & $6.327 \mathrm{E}+04$ & $7.001 \mathrm{E}+04$ & & $7.404 \mathrm{E}+04$ & & $7.404 \mathrm{E}+04$ & $7.474 \mathrm{E}+04$ \\
\hline Sodium Hydroxide & $2.572 \mathrm{E}+04$ & $6.114 \mathrm{E}+03$ & $3.253 \mathrm{E}+05$ & $3.563 \mathrm{E}+05$ & & $3.540 \mathrm{E}+05$ & & $3.540 \mathrm{E}+05$ & $3.430 \mathrm{E}+05$ \\
\hline Naphthalene & & $2.243 E-06$ & $4.188 E+00$ & $4.188 E+00$ & & $4.188 E+00$ & $4.188 E+00$ & $1.925 E-04$ & $1.925 E-04$ \\
\hline Sodium Aluminosilicate Gel & $2.920 \mathrm{E}+03$ & \begin{tabular}{|l|l}
$3.466 \mathrm{E}+02$ \\
\end{tabular} & & $3.894 \mathrm{E}+03$ & & $3.759 \mathrm{E}+03$ & & $3.759 \mathrm{E}+03$ & $3.784 \mathrm{E}+03$ \\
\hline Nickel (II) Oxalate & & $8.077 \mathrm{E}+00$ & & $8.077 \mathrm{E}+00$ & & $8.077 \mathrm{E}+00$ & & $8.077 \mathrm{E}+00$ & $8.077 \mathrm{E}+00$ \\
\hline Oxygen & & $9.698 \mathrm{E}-07$ & & $9.698 \mathrm{E}-07$ & $8.994 \mathrm{E}+02$ & $8.994 \mathrm{E}+02$ & $8.995 \mathrm{E}+02$ & $7.675 \mathrm{E}-05$ & $7.675 \mathrm{E}-05$ \\
\hline Phosphorus Pentoxide & & $8.180 \mathrm{E}+00$ & $6.391 \mathrm{E}+02$ & $6.473 \mathrm{E}+02$ & & $6.473 \mathrm{E}+02$ & & $6.473 \mathrm{E}+02$ & $6.473 \mathrm{E}+02$ \\
\hline PYRENE & & \begin{tabular}{|l|}
$6.996 E-05$ \\
\end{tabular} & $4.202 E+00$ & $4.202 E+00$ & & $4.202 E+00$ & $4.090 E+00$ & $1.115 E-01$ & $1.115 E-01$ \\
\hline Silicon Dioxide & $3.339 \mathrm{E}+02$ & $3.496 \mathrm{E}+00$ & & $1.102 \mathrm{E}+02$ & & $1.589 \mathrm{E}+02$ & & $1.589 \mathrm{E}+02$ & $1.502 \mathrm{E}+02$ \\
\hline Sulfur Trioxide & $5.781 \mathrm{E}+02$ & $1.074 \mathrm{E}+02$ & $4.702 \mathrm{E}+04$ & $4.770 \mathrm{E}+04$ & & $4.770 \mathrm{E}+04$ & & $4.770 \mathrm{E}+04$ & $4.047 \mathrm{E}+04$ \\
\hline 1,2,4-trichlorobenzene & & \begin{tabular}{|l|}
$4.735 E-07$ \\
\end{tabular} & $4.193 E+00$ & $4.193 E+00$ & & $4.193 E+00$ & $4.193 E+00$ & $5.306 E-05$ & $5.306 E-05$ \\
\hline 1,2,3-trichloropropane & & \begin{tabular}{|l|}
$3.338 E-06$ \\
\end{tabular} & $4.198 E+00$ & $4.198 E+00$ & & $4.198 E+00$ & $4.198 E+00$ & $2.657 E-04$ & $2.657 E-04$ \\
\hline TOLUENE & & \begin{tabular}{|l|}
$2.940 E-07$ \\
\end{tabular} & $4.194 E+00$ & $4.194 E+00$ & & $4.194 E+00$ & $4.194 E+00$ & $2.351 E-05$ & $2.351 E-05$ \\
\hline Zinc Dichloride Pentoxide & $9.198 \mathrm{E}+00$ & $5.119 \mathrm{E}+00$ & & & & & & & \\
\hline Zinc Oxalate & & $5.908 \mathrm{E}+00$ & & $1.154 \mathrm{E}+03$ & & $1.154 \mathrm{E}+03$ & & $1.154 \mathrm{E}+03$ & $1.154 \mathrm{E}+03$ \\
\hline Zinc Hydroxide & $7.281 \mathrm{E}+02$ & & & & & & & & \\
\hline Zirconium EDTA & & $7.440 \mathrm{E}-01$ & & $6.570 \mathrm{E}+00$ & & $7.143 \mathrm{E}+00$ & & $7.143 \mathrm{E}+00$ & $3.098 \mathrm{E}+01$ \\
\hline Zirconium Oxide & & $9.818 \mathrm{E}+00$ & & $7.926 \mathrm{E}+00$ & & $7.740 \mathrm{E}+00$ & & $7.740 \mathrm{E}+00$ & \\
\hline Total $\mathrm{g} / \mathrm{hr}$ & $3.438 \mathrm{E}+06$ & $1.528 \mathrm{E}+05$ & $4.012 \mathrm{E}+06$ & $7.603 E+06$ & $3.889 E+03$ & $7.607 \mathrm{E}+06$ & $5.796 \mathrm{E}+06$ & $1.811 \mathrm{E}+06$ & $1.811 \mathrm{E}+06$ \\
\hline Volume, L/hr & $3.405 \mathrm{E}+03$ & $1.436 \mathrm{E}+02$ & $3.551 \mathrm{E}+03$ & $6.703 \mathrm{E}+07$ & $3.307 \mathrm{E}+03$ & $8.341 \mathrm{E}+07$ & $8.341 \mathrm{E}+07$ & $1.368 \mathrm{E}+03$ & $1.319 \mathrm{E}+03$ \\
\hline Enthalpy, cal/hr & $-1.289 E+10$ & $-5.506 \mathrm{E}+08$ & $-1.404 \mathrm{E}+10$ & $-2.450 \mathrm{E}+10$ & $-6.275 \mathrm{E}+04$ & $-2.402 \mathrm{E}+10$ & $-1.852 \mathrm{E}+10$ & $-5.498 \mathrm{E}+09$ & $-5.522 \mathrm{E}+09$ \\
\hline Vapor fraction & & & & \begin{tabular}{|l|}
$6.967 \mathrm{E}-01$ \\
\end{tabular} & $1.000 \mathrm{E}+00$ & $8.170 \mathrm{E}-01$ & $1.000 \mathrm{E}+00$ & & \\
\hline Solid fraction & $1.181 \mathrm{E}-04$ & 1.707E-04 & $2.237 \mathrm{E}-04$ & \begin{tabular}{|l|}
$1.349 \mathrm{E}-04$ \\
\end{tabular} & & $2.539 \mathrm{E}-04$ & & $1.387 \mathrm{E}-03$ & $2.838 \mathrm{E}-03$ \\
\hline Organic fraction & & $2.209 \mathrm{E}-10$ & 4.886E-07 & 6.257E-08 & & $5.873 \mathrm{E}-08$ & & $3.210 \mathrm{E}-07$ & 3.139E-07 \\
\hline Osmotic Pres, atm & $1.256 \mathrm{E}+01$ & $4.822 \mathrm{E}+01$ & $9.071 \mathrm{E}+01$ & $1.801 \mathrm{E}+02$ & & $2.724 \mathrm{E}+02$ & & $2.724 \mathrm{E}+02$ & $2.716 \mathrm{E}+02$ \\
\hline \multicolumn{10}{|l|}{ Redox Pot, volts } \\
\hline E-Con, 1/ohm-cm & $2.639 \mathrm{E}-02$ & $9.183 \mathrm{E}-02$ & $1.378 \mathrm{E}-01$ & $2.765 \mathrm{E}-01$ & & $3.799 \mathrm{E}-01$ & & $3.799 \mathrm{E}-01$ & $2.596 \mathrm{E}-01$ \\
\hline E-Con, cm2/ohm-mol & $9.004 \mathrm{E}+01$ & $4.315 \mathrm{E}+01$ & $6.830 \mathrm{E}+01$ & $3.659 \mathrm{E}+01$ & & $3.077 \mathrm{E}+01$ & & $3.077 \mathrm{E}+01$ & $2.052 \mathrm{E}+01$ \\
\hline Abs Visc, cP & 9.062E-01 & $1.063 \mathrm{E}+00$ & $1.494 \mathrm{E}+00$ & $1.449 \mathrm{E}+00$ & & $2.382 \mathrm{E}+00$ & & $2.382 \mathrm{E}+00$ & $4.188 \mathrm{E}+00$ \\
\hline Rel Visc & $1.017 \mathrm{E}+00$ & $1.194 \mathrm{E}+00$ & $1.678 \mathrm{E}+00$ & $2.648 \mathrm{E}+00$ & & $4.353 \mathrm{E}+00$ & & $4.353 \mathrm{E}+00$ & $4.701 \mathrm{E}+00$ \\
\hline Ionic Strength & \begin{tabular}{|l|}
$2.815 \mathrm{E}-01$ \\
\end{tabular} & $1.376 \mathrm{E}+00$ & $3.062 \mathrm{E}+00$ & $5.935 \mathrm{E}+00$ & & $1.052 \mathrm{E}+01$ & & $1.052 \mathrm{E}+01$ & $9.970 \mathrm{E}+00$ \\
\hline
\end{tabular}


WSRC'-'IR-2UUZ-U0550, Rev. 1

SRT-RPP-2002-0027.

Table A- 2. Waste Feed Evaporator Offgas Model Output for UF1 Filtrate Density of $1.35 \mathrm{~g} / \mathrm{ml}$ (cont'd) ${ }^{8}$

\begin{tabular}{|c|c|c|c|c|c|c|c|c|c|}
\hline 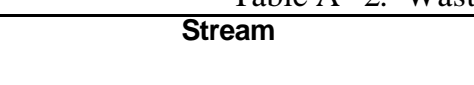 & $\begin{array}{c}\text { UF1 } \\
\text { Filtrate }\end{array}$ & UF1 Slurry & PC Vapor & $\begin{array}{c}\text { PC } \\
\text { Condensate }\end{array}$ & $\begin{array}{c}\text { PC Control } \\
\text { Air }\end{array}$ & $\begin{array}{c}\text { PC Vap + } \\
\text { CtrlAir }\end{array}$ & $\begin{array}{l}\text { Super } \\
\text { Steam } 1\end{array}$ & $\begin{array}{c}\text { Stm Eject } 1 \\
\text { Out }\end{array}$ & IC Vapor \\
\hline Phase & Mixed & Mixed & Vapor & Mixed & Vapor & Vapor & Aqueous & Mixed & Vapor \\
\hline Temperature, C & 25 & 25 & 40 & 40 & 25 & 34.7123 & 133 & 58.2382 & 40 \\
\hline Pressure, atm & 1 & 1 & 0.103151 & 0.103151 & 1 & \begin{tabular}{|l|}
0.0979798 \\
\end{tabular} & 8.41674 & 0.294007 & 0.279311 \\
\hline $\mathrm{pH}$ & 14.034 & 14.034 & & 6.72036 & & & 5.90373 & 6.25211 & \\
\hline Total $\mathrm{mol} / \mathrm{hr}$ & 66180.7 & 5671.62 & 460.449 & 321187 & 270.36 & 730.809 & 2517.81 & 3248.62 & 545.525 \\
\hline Flow Units & $g / h r$ & $\mathrm{~g} / \mathrm{hr}$ & $\mathrm{g} / \mathrm{hr}$ & $g / h r$ & $g / h r$ & $\mathrm{~g} / \mathrm{hr}$ & $\mathrm{g} / \mathrm{hr}$ & $\mathrm{g} / \mathrm{hr}$ & $\mathrm{g} / \mathrm{hr}$ \\
\hline Acetic Acid, Dimer & & & $6.816 \mathrm{E}-26$ & & & & & & \\
\hline Acetic Acid & & & $2.022 \mathrm{E}-11$ & $2.715 \mathrm{E}-06$ & & & & & \\
\hline Aluminum Oxide & $4.375 \mathrm{E}+03$ & $3.615 \mathrm{E}+02$ & & & & & & & \\
\hline Aldrin & $4.038 E-01$ & \begin{tabular}{|l|}
$3.336 E-02$ \\
\end{tabular} & $9.165 E-03$ & $3.749 E+00$ & & $9.165 E-03$ & & $9.165 E-03$ & $3.186 E-03$ \\
\hline Aluminum Hydrogen EDTA & $1.097 \mathrm{E}+03$ & $9.067 \mathrm{E}+01$ & & & & & & & \\
\hline \multicolumn{10}{|l|}{ Aluminum Hydroxide } \\
\hline Benzene & $2.593 E-05$ & 2.142E-06 & $3.712 E+00$ & $4.812 E-01$ & & $3.712 E+00$ & & $3.712 E+00$ & $3.703 E+00$ \\
\hline Bis(2-ethylhexyl)phthalate (BEHP) & $1.803 E+00$ & \begin{tabular}{|l|}
$1.489 E-01$ \\
\end{tabular} & $3.838 E-04$ & $2.242 E+00$ & & \begin{tabular}{|l|}
$3.838 E-04$ \\
\end{tabular} & & $3.838 E-04$ & $1.342 E-05$ \\
\hline Benzo(a)pyrene (BaP) & $5.716 E-04$ & $4.181 E+00$ & & & & & & & \\
\hline Phenol & $3.921 E+00$ & \begin{tabular}{|l|}
$3.239 E-01$ \\
\end{tabular} & $6.063 E-06$ & $4.506 E-03$ & & $6.063 E-06$ & & $6.063 E-06$ & $3.975 E-07$ \\
\hline \multicolumn{10}{|l|}{ Calcium Chloride Oxide } \\
\hline Calcium Oxalate & $5.788 \mathrm{E}+02$ & $4.782 \mathrm{E}+01$ & & & & & & & \\
\hline \multicolumn{10}{|l|}{ Calcium Oxalate Monohydrate } \\
\hline \multicolumn{10}{|l|}{ Calcium Carbonate } \\
\hline \multicolumn{10}{|l|}{ Calcium Citrate } \\
\hline \multicolumn{10}{|l|}{ Calcium Fluoride } \\
\hline Pentachlorophenol & $1.018 \mathrm{E}-01$ & $8.408 E-03$ & $5.461 E-03$ & $4.082 E+00$ & & $5.461 E-03$ & & $5.461 E-03$ & $3.598 E-04$ \\
\hline Hexachlorobenzene & $1.364 E-03$ & $4.186 E+00$ & & & & & & & \\
\hline Chlorobenzene & \begin{tabular}{|l}
$5.586 E-05$ \\
\end{tabular} & \begin{tabular}{|l|}
$4.615 E-06$ \\
\end{tabular} & $3.346 E+00$ & $8.446 E-01$ & & $3.346 E+00$ & & $3.346 E+00$ & $3.330 E+00$ \\
\hline 2-Chloronaphthalene & $2.253 E-04$ & $1.861 E-05$ & $1.950 E+00$ & $2.244 E+00$ & & $1.950 E+00$ & & $1.950 E+00$ & $1.909 E+00$ \\
\hline Carbon Dioxide & $7.341 \mathrm{E}+04$ & $6.065 \mathrm{E}+03$ & $4.528 \mathrm{E}-02$ & $5.170 \mathrm{E}-03$ & $3.897 \mathrm{E}+00$ & $3.942 \mathrm{E}+00$ & & $3.942 \mathrm{E}+00$ & $3.939 E+00$ \\
\hline Cesium Acetate & $1.845 \mathrm{E}+02$ & $1.524 \mathrm{E}+01$ & & & & & & & \\
\hline Dibenz[a,h]anthracene & $3.860 E+00$ & \begin{tabular}{|l|}
$3.189 E-01$ \\
\end{tabular} & $1.052 E-08$ & $1.244 E-02$ & & $1.052 E-08$ & & $1.052 E-08$ & $2.330 E-12$ \\
\hline 1,2-dibromoetha ne & $1.335 \mathrm{E}-03$ & $1.103 E-04$ & $1.365 E+01$ & $1.441 E+01$ & & $1.365 E+01$ & & $1.365 E+01$ & $1.338 E+01$ \\
\hline \multirow{2}{*}{\multicolumn{10}{|c|}{ Iron (III) Chloride }} \\
\hline & & & & & & & & & \\
\hline Iron (III) Hydrogen EDTA & $5.537 \mathrm{E}+02$ & $4.574 \mathrm{E}+01$ & & & & & & & \\
\hline \multicolumn{10}{|l|}{ Iron (III) Hydroxide } \\
\hline \multicolumn{10}{|l|}{ Formic Acid, dimer } \\
\hline \multicolumn{10}{|l|}{ Hydrofluoride, dimer } \\
\hline 2,2'-Iminobisacetic acid & $1.059 \mathrm{E}+03$ & $8.752 \mathrm{E}+01$ & & & & & & & \\
\hline Water & $9.246 \mathrm{E}+05$ & $7.639 \mathrm{E}+04$ & $5.875 \mathrm{E}+03$ & $5.786 \mathrm{E}+06$ & $3.638 \mathrm{E}+01$ & $5.912 \mathrm{E}+03$ & $4.536 \mathrm{E}+04$ & $5.127 \mathrm{E}+04$ & $2.574 \mathrm{E}+03$ \\
\hline \multicolumn{10}{|l|}{ Sulfuric Acid } \\
\hline Hydrogen Chloride & $9.617 \mathrm{E}+03$ & $7.945 \mathrm{E}+02$ & $1.663 \mathrm{E}-23$ & & & & & & \\
\hline Formic Acid & $3.705 \mathrm{E}+02$ & $3.061 \mathrm{E}+01$ & $1.449 \mathrm{E}-13$ & $3.091 \mathrm{E}-07$ & & & & & \\
\hline Hydrofluoric Acid & $1.242 \mathrm{E}+03$ & $1.026 \mathrm{E}+02$ & $4.954 \mathrm{E}-15$ & $1.450 \mathrm{E}-07$ & & & & & \\
\hline \multicolumn{10}{|l|}{ Mercury (II) Chloride } \\
\hline Mercury (elemental) & $1.275 \mathrm{E}-04$ & $1.053 \mathrm{E}-05$ & $7.784 \mathrm{E}+00$ & $5.596 \mathrm{E}+01$ & & $7.784 \mathrm{E}+00$ & & $7.784 \mathrm{E}+00$ & $3.408 \mathrm{E}+00$ \\
\hline Mercury (II) Oxide & $1.794 \mathrm{E}+01$ & $5.084 \mathrm{E}+01$ & & & & & & & \\
\hline Nitrous (III) Acid & $8.618 \mathrm{E}+04$ & $7.120 \mathrm{E}+03$ & $2.825 \mathrm{E}-07$ & $6.469 \mathrm{E}-03$ & & $2.825 \mathrm{E}-07$ & & $2.825 \mathrm{E}-07$ & 2.567E-09 \\
\hline Nitric Acid & $6.145 \mathrm{E}+04$ & $5.077 \mathrm{E}+03$ & & & & & & & \\
\hline Hexachlorobutadiene & $5.123 E-06$ & 4.233E-07 & $4.165 E+00$ & $3.471 E-02$ & & $4.165 E+00$ & & $4.165 E+00$ & $4.164 E+00$ \\
\hline Potassium Citrate & $2.641 \mathrm{E}+03$ & \begin{tabular}{|l|}
$2.182 \mathrm{E}+02$ \\
\end{tabular} & & & & & & & \\
\hline Potassium Chloride & $3.494 \mathrm{E}+04$ & $2.887 \mathrm{E}+03$ & & & & & & & \\
\hline \multicolumn{10}{|l|}{ Potassium Nitrate $(\mathrm{VI})$} \\
\hline gamma-BHC (Lindane) & $5.685 E-03$ & 4.697E-04 & $2.139 E-01$ & $3.989 E+00$ & & $2.139 E-01$ & & $2.139 E-01$ & $1.602 E-01$ \\
\hline 4-methyl-2-pentanone (MIBK) & 6.187E-04 & \begin{tabular}{|l|}
$5.111 E-05$ \\
\end{tabular} & $8.521 E-01$ & $3.344 E+00$ & & $8.521 E-01$ & & $8.521 E-01$ & 7.924E-01 \\
\hline Magnesium Oxalate & \begin{tabular}{|l}
$1.887 \mathrm{E}-03$ \\
\end{tabular} & \begin{tabular}{|l|}
$1.559 \mathrm{E}-04$ \\
\end{tabular} & & & & & & & \\
\hline Magnesium Hydroxide & & $2.392 \mathrm{E}+02$ & & & & & & & \\
\hline Manganese (II) Oxalate & $2.474 \mathrm{E}+00$ & \begin{tabular}{|l|}
$2.044 \mathrm{E}-01$ \\
\end{tabular} & & & & & & & \\
\hline \multicolumn{10}{|l|}{ Manganese (II) Hydroxide } \\
\hline Nitrogen & $1.274 \mathrm{E}-04$ & $1.053 \mathrm{E}-05$ & $2.968 \mathrm{E}+03$ & $2.114 \mathrm{E}+00$ & $5.940 \mathrm{E}+03$ & $8.908 \mathrm{E}+03$ & & $8.908 \mathrm{E}+03$ & $8.907 \mathrm{E}+03$ \\
\hline
\end{tabular}


WSRC-'IR-2UU2-00550, Rev. I

SRT-RPP-2002-0027.

\begin{tabular}{|c|c|c|c|c|c|c|c|c|c|}
\hline Stream & $\begin{array}{c}\text { UF1 } \\
\text { Filtrate }\end{array}$ & UF1 Slurry & PC Vapor & $\begin{array}{c}\mathrm{PC} \\
\text { Condensate }\end{array}$ & $\begin{array}{c}P C \text { Control } \\
\text { Air }\end{array}$ & $\underset{\text { CtrlAir }}{\text { PC Vap + }}$ & $\begin{array}{c}\text { Super } \\
\text { Steam } 1\end{array}$ & $\begin{array}{c}\text { Stm Eject } 1 \\
\text { Out }\end{array}$ & IC Vapor \\
\hline Sodium Oxalate & $1.480 \mathrm{E}+03$ & $1.191 \mathrm{E}+04$ & & & & & & & \\
\hline Sodium Chromate (VI) & $7.811 \mathrm{E}+03$ & $6.453 \mathrm{E}+02$ & & & & & & & \\
\hline Sodium Floride Sulfate & & $1.662 \mathrm{E}+04$ & & & & & & & \\
\hline Sodium Acetate & 1.717E+02 & $1.418 \mathrm{E}+01$ & & & & & & & \\
\hline Sodium Glycolate & $4.263 E+03$ & $3.522 \mathrm{E}+02$ & & & & & & & \\
\hline Sodium Nitrite & $6.903 E+04$ & $5.703 \mathrm{E}+03$ & & & & & & & \\
\hline Sodium Hydroxide & $3.168 \mathrm{E}+05$ & $2.617 \mathrm{E}+04$ & & & & & & & \\
\hline Naphthalene & 1.779E-04 & \begin{tabular}{|l}
$1.469 E-05$ \\
\end{tabular} & $2.336 E+00$ & $1.853 E+00$ & & $2.336 E+00$ & & $2.336 E+00$ & $2.3 C$ \\
\hline Sodium Aluminosilicate Gel & & $3.784 \mathrm{E}+03$ & & & & & & & \\
\hline Nickel (II) Oxalate & $7.461 \mathrm{E}+00$ & \begin{tabular}{|l|}
$6.164 \mathrm{E}-01$ \\
\end{tabular} & & & & & & & \\
\hline Oxygen & $7.090 \mathrm{E}-05$ & 5.857E-06 & $8.982 \mathrm{E}+02$ & $1.201 \mathrm{E}+00$ & $1.799 \mathrm{E}+03$ & $2.697 \mathrm{E}+03$ & & $2.697 \mathrm{E}+03$ & $2.697 \mathrm{E}+03$ \\
\hline Phosphorus Pentoxide & $5.979 \mathrm{E}+02$ & $4.940 \mathrm{E}+01$ & & & & & & & \\
\hline PYRENE & $1.030 E-01$ & $8.513 E-03$ & $4.345 E-02$ & $4.047 E+00$ & & $4.345 E-02$ & & 4.345E-02 & $2.363 E-02$ \\
\hline Silicon Dioxide & $1.387 \mathrm{E}+02$ & $1.146 \mathrm{E}+01$ & & & & & & & \\
\hline Sulfur Trioxide & $3.738 \mathrm{E}+04$ & $3.088 \mathrm{E}+03$ & & & & & & & \\
\hline 1,2,4-trichlorobenzene & 4.902E-05 & $4.050 E-06$ & $3.372 E+00$ & $8.206 E-01$ & & $3.372 E+00$ & & $3.372 E+00$ & $3.357 E+00$ \\
\hline 1,2,3-trichloropropane & 2.454E-04 & 2.027E-05 & $1.686 E+00$ & $2.512 E+00$ & & $1.686 E+00$ & & $1.686 E+00$ & $1.639 E+00$ \\
\hline TOLUENE & $2.172 E-05$ & $1.794 E-06$ & $3.767 E+00$ & $4.263 E-01$ & & $3.767 E+00$ & & $3.768 E+00$ & $3.759 E+00$ \\
\hline Zinc Dichloride Pentoxide & & & & & & & & & \\
\hline Zinc Oxalate & $1.066 \mathrm{E}+03$ & $8.805 \mathrm{E}+01$ & & & & & & & \\
\hline Zinc Hydroxide & & & & & & & & & \\
\hline Zirconium EDTA & $2.861 \mathrm{E}+01$ & $2.364 \mathrm{E}+00$ & & & & & & & \\
\hline Zirconium Oxide & & & & & & & & & \\
\hline Total g/hr & $1.641 \mathrm{E}+06$ & $1.695 \mathrm{E}+05$ & $9.788 \mathrm{E}+03$ & $5.786 \mathrm{E}+06$ & $7.779 \mathrm{E}+03$ & $1.757 \mathrm{E}+04$ & $4.536 \mathrm{E}+04$ & $6.293 \mathrm{E}+04$ & $1.422 \mathrm{E}+04$ \\
\hline Volume, L/hr & $1.216 \mathrm{E}+03$ & $1.039 \mathrm{E}+02$ & $1.146 \mathrm{E}+05$ & $5.833 \mathrm{E}+03$ & \begin{tabular}{|l|}
$6.613 \mathrm{E}+03$ \\
\end{tabular} & $1.884 \mathrm{E}+05$ & $4.866 \mathrm{E}+01$ & $9.735 \mathrm{E}+04$ & $5.016 \mathrm{E}+04$ \\
\hline Enthalpy, cal/hr & $-5.017 \mathrm{E}+09$ & $-5.049 \mathrm{E}+08$ & $-1.879 \mathrm{E}+07$ & $-2.186 \mathrm{E}+10$ & \begin{tabular}{|l|}
$-1.255 E+05$ \\
\end{tabular} & $-1.892 \mathrm{E}+07$ & $-1.671 \mathrm{E}+08$ & $-1.860 \mathrm{E}+08$ & $-8.207 \mathrm{E}+06$ \\
\hline Vapor fraction & & & $1.000 \mathrm{E}+00$ & & $1.000 \mathrm{E}+00$ & $1.000 \mathrm{E}+00$ & & $3.244 \mathrm{E}-01$ & $1.000 \mathrm{E}+00$ \\
\hline Solid fraction & & $3.596 \mathrm{E}-02$ & & $8.637 \mathrm{E}-07$ & & & & & \\
\hline Organic fraction & $3.148 \mathrm{E}-07$ & \begin{tabular}{|l}
$3.035 \mathrm{E}-07$ \\
\end{tabular} & & $9.314 \mathrm{E}-08$ & & & & & \\
\hline Osmotic Pres, atm & $2.716 \mathrm{E}+02$ & \begin{tabular}{|l|}
$2.716 \mathrm{E}+02$ \\
\end{tabular} & & $1.533 \mathrm{E}-03$ & & & & $2.291 \mathrm{E}-03$ & \\
\hline Redox Pot, volts & & & & & & & & & \\
\hline E-Con, 1/ohm-cm & & & & $1.189 \mathrm{E}-07$ & & & $1.478 \mathrm{E}-06$ & $3.510 \mathrm{E}-07$ & \\
\hline E-Con, cm2/ohm-mol & & & & $5.139 \mathrm{E}-03$ & & & & $3.502 \mathrm{E}-05$ & \\
\hline Abs Visc, cP & & & & $6.536 \mathrm{E}-01$ & & & $2.077 \mathrm{E}-01$ & $4.792 \mathrm{E}-01$ & \\
\hline Rel Visc & & & & $1.000 \mathrm{E}+00$ & & & $1.000 \mathrm{E}+00$ & $1.000 \mathrm{E}+00$ & \\
\hline Ionic Strength & $9.970 \mathrm{E}+00$ & $9.970 \mathrm{E}+00$ & & $1.905 \mathrm{E}-07$ & & & $1.250 \mathrm{E}-06$ & $5.602 \mathrm{E}-07$ & \\
\hline
\end{tabular}


Table A- 2. Waste Feed Evaporator Offgas Model Output for UF1 Filtrate Density of $1.35 \mathrm{~g} / \mathrm{ml}$ (cont'd) ${ }^{8}$

\begin{tabular}{|c|c|c|c|c|c|c|c|c|}
\hline Stream & $\begin{array}{c}\text { IC } \\
\text { Condensate }\end{array}$ & $\begin{array}{c}\text { Super } \\
\text { Steam } 2\end{array}$ & $\begin{array}{c}\text { Stm Eject } 2 \\
\text { Out }\end{array}$ & AC Vapor & $\begin{array}{c}\mathrm{AC} \\
\text { Condensate }\end{array}$ & $\begin{array}{l}\text { Demist } \\
\text { Vapor }\end{array}$ & $\begin{array}{l}\text { Demist } \\
\text { Liquid }\end{array}$ & $\begin{array}{c}\text { Evap Cond } \\
\text { Tk Out }\end{array}$ \\
\hline Phase & Mixed & Aqueous & Mixed & Vapor & Mixed & Mixed & Mixed & Mixed \\
\hline Temperature, $\mathrm{C}$ & 40 & 133 & 76.7334 & 40 & 40 & 40 & 40 & 25 \\
\hline Pressure, atm & 0.279311 & 8.41674 & 0.837932 & 0.796018 & 0.796018 & 0.985242 & 0.985242 & 1 \\
\hline $\mathrm{pH}$ & 6.11444 & 5.90373 & 6.03692 & & 5.85076 & 5.80105 & 5.80105 & 6.82672 \\
\hline Total mol/hr & 2703.1 & 2517.81 & 3063.34 & 443.492 & 2619.85 & 441.835 & 1.65736 & 326512 \\
\hline Flow Units & $\mathrm{g} / \mathrm{hr}$ & $\mathrm{g} / \mathrm{hr}$ & $\mathrm{g} / \mathrm{hr}$ & $\mathrm{g} / \mathrm{hr}$ & $\mathrm{g} / \mathrm{hr}$ & $g / h r$ & $\mathrm{~g} / \mathrm{hr}$ & $g / h r$ \\
\hline \multicolumn{9}{|l|}{ Acetic Acid, Dimer } \\
\hline Acetic Acid & & & & & & & & $2.715 \mathrm{E}-06$ \\
\hline \multicolumn{9}{|l|}{ Aluminum Oxide } \\
\hline Aldrin & 5.979E-03 & & $3.186 E-03$ & 4.442E-04 & 2.742E-03 & 4.009E-04 & 4.332E-05 & $3.757 E+00$ \\
\hline \multicolumn{9}{|l|}{ Aluminum Hydrogen EDTA } \\
\hline \multicolumn{9}{|l|}{ Aluminum Hydroxide } \\
\hline Benzene & 9.217E-03 & & $3.703 E+00$ & $3.672 E+00$ & $3.089 E-02$ & $3.672 E+00$ & $2.469 E-05$ & $5.213 E-01$ \\
\hline Bis(2-ethylhexyl)phthalate (BEHP) & $3.704 E-04$ & & $1.342 E-05$ & 1.541E-07 & 1.327E-05 & 1.282E-07 & $2.588 E-08$ & $2.243 E+00$ \\
\hline \multicolumn{9}{|l|}{ Benzo(a)pyrene (BaP) } \\
\hline Phenol & $5.665 E-06$ & & $3.975 E-07$ & 8.095E-09 & $3.894 E-07$ & $7.836 E-09$ & $2.593 E-10$ & $4.512 E-03$ \\
\hline \multicolumn{9}{|l|}{ Calcium Chloride Oxide } \\
\hline \multicolumn{9}{|l|}{ Calcium Oxalate } \\
\hline \multicolumn{9}{|l|}{ Calcium Oxalate Monohydrate } \\
\hline \multicolumn{9}{|l|}{ Calcium Carbonate } \\
\hline \multicolumn{9}{|l|}{ Calcium Citrate } \\
\hline \multicolumn{9}{|l|}{ Calcium Fluoride } \\
\hline Pentachlorophenol & 5.101E-03 & & 3.598E-04 & 7.394E-06 & 3.524E-04 & 7.066E-06 & 3.279E-07 & $4.087 E+00$ \\
\hline \multicolumn{9}{|l|}{ Hexachlorobenzene } \\
\hline Chlorobenzene & $1.613 E-02$ & & $3.330 E+00$ & $3.276 E+00$ & $5.361 E-02$ & $3.276 E+00$ & 4.321E-05 & 9.144E-01 \\
\hline 2-Chloronaphthalene & 4.123E-02 & & $1.909 E+00$ & $1.779 E+00$ & $1.298 E-01$ & $1.779 E+00$ & 1.930E-04 & $2.415 E+00$ \\
\hline Carbon Dioxide & $3.887 \mathrm{E}-03$ & & $3.939 \mathrm{E}+00$ & $3.928 \mathrm{E}+00$ & 1.073E-02 & $3.928 \mathrm{E}+00$ & 8.307E-06 & $1.980 \mathrm{E}-02$ \\
\hline \multicolumn{9}{|l|}{ Cesium Acetate } \\
\hline Dibenz[a,h]anthracene & $1.049 E-08$ & & & & & & & $1.244 E-02$ \\
\hline 1,2-dibromoethane & $2.710 E-01$ & & $1.338 E+01$ & $1.253 E+01$ & $8.574 E-01$ & $1.253 E+01$ & 6.847E-04 & $1.554 E+01$ \\
\hline Diethyl phthalate & $1.541 E-03$ & & $3.217 E-05$ & $1.988 E-07$ & $3.198 E-05$ & 1.832E-07 & $1.556 E-08$ & $3.941 E+00$ \\
\hline \multicolumn{9}{|l|}{ Iron (III) Chloride } \\
\hline \multicolumn{9}{|l|}{ Iron (III) Hydrogen EDTA } \\
\hline Iron (III) Hydroxide & & & & & & & & \\
\hline Formic Acid, dimer & & & & & & & & \\
\hline Hydrofluoride, dimer & & & & & & & & \\
\hline 2,2'-Iminobisacetic acid & & & & & & & & \\
\hline Water & $4.870 \mathrm{E}+04$ & $4.536 \mathrm{E}+04$ & $4.793 \mathrm{E}+04$ & $7.368 \mathrm{E}+02$ & $4.720 \mathrm{E}+04$ & $7.070 \mathrm{E}+02$ & $2.984 \mathrm{E}+01$ & $5.882 \mathrm{E}+06$ \\
\hline Sulfuric Acid & & & & & & & & \\
\hline Hydrogen Chloride & & & & & & & & \\
\hline Formic Acid & & & & & & & & 3.091E-07 \\
\hline Hydrofluoric Acid & & & & & & & & $1.450 \mathrm{E}-07$ \\
\hline Mercury (II) Chloride & & & & & & & & \\
\hline Mercury (elemental) & $4.376 \mathrm{E}+00$ & & $3.408 \mathrm{E}+00$ & $9.731 \mathrm{E}-01$ & $2.435 \mathrm{E}+00$ & 7.715E-01 & $2.016 \mathrm{E}-01$ & $6.297 \mathrm{E}+01$ \\
\hline Mercury (II) Oxide & & & & & & & & \\
\hline Nitrous (III) Acid & $2.799 \mathrm{E}-07$ & & 2.567E-09 & $1.270 \mathrm{E}-11$ & 2.554E-09 & & & $6.469 \mathrm{E}-03$ \\
\hline Nitric Acid & & & & & & & & \\
\hline Hexachlorobutadiene & $5.448 E-04$ & & $4.164 E+00$ & $4.163 E+00$ & $1.838 E-03$ & $4.163 E+00$ & $6.208 E-06$ & $3.710 E-02$ \\
\hline Potassium Citrate & & & & & & & & \\
\hline Potassium Chloride & & & & & & & & \\
\hline Potassium Nitrate (VI) & & & & & & & & \\
\hline gamma-BHC (Lindane) & 5.370E-02 & & 1.602E-01 & 7.527E-02 & $8.493 E-02$ & $7.511 E-02$ & $1.596 E-04$ & $4.128 E+00$ \\
\hline 4-methyl-2-pentanone (MIBK) & $5.968 E-02$ & & 7.924E-01 & $6.316 E-01$ & $1.607 E-01$ & $6.315 E-01$ & $1.279 E-04$ & $3.564 E+00$ \\
\hline Magnesium Oxalate & & & & & & & & \\
\hline Magnesium Hydroxide & & & & & & & & \\
\hline Manganese (II) Oxalate & & & & & & & & \\
\hline Manganese (II) Hydroxide & & & & & & & & \\
\hline Nitrogen & $1.220 \mathrm{E}-01$ & & 8.907E+03 & $8.907 \mathrm{E}+03$ & 4.142E-01 & $8.907 \mathrm{E}+03$ & 3.304E-04 & $2.651 \mathrm{E}+00$ \\
\hline
\end{tabular}


WSKC-'IR-2002-00550, Kev. I SRT-RPP-2002-0027.

\begin{tabular}{|c|c|c|c|c|c|c|c|c|}
\hline Stream & $\begin{array}{c}\text { IC } \\
\text { Condensate }\end{array}$ & $\begin{array}{c}\text { Super } \\
\text { Steam } 2\end{array}$ & $\begin{array}{c}\text { Stm Eject } 2 \\
\text { Out }\end{array}$ & AC Vapor & $\begin{array}{c}\text { AC } \\
\text { Condensate }\end{array}$ & $\begin{array}{l}\text { Demist } \\
\text { Vapor }\end{array}$ & $\begin{array}{l}\text { Demist } \\
\text { Liquid }\end{array}$ & $\begin{array}{l}\text { Evap Cond } \\
\text { Tk Out }\end{array}$ \\
\hline \multicolumn{9}{|l|}{ Sodium Oxalate } \\
\hline \multicolumn{9}{|l|}{ Sodium Chromate (VI) } \\
\hline \multicolumn{9}{|l|}{ Sodium Acetate } \\
\hline \multicolumn{9}{|l|}{ Sodium Glycolate } \\
\hline \multicolumn{9}{|l|}{ Sodium Nitrite } \\
\hline Naphthalene & $3.480 E-02$ & & $2.301 E+00$ & $2.189 E+00$ & 1.117E-01 & $2.189 E+00$ & $1.042 E-04$ & $1.999 E+00$ \\
\hline \multicolumn{9}{|l|}{ Sodium Aluminosilicate Gel } \\
\hline \multicolumn{9}{|l|}{ Nickel (II) Oxalate } \\
\hline Oxygen & $6.930 \mathrm{E}-02$ & & $2.697 \mathrm{E}+03$ & $2.697 \mathrm{E}+03$ & $2.352 \mathrm{E}-01$ & $2.697 \mathrm{E}+03$ & $1.876 \mathrm{E}-04$ & $1.505 \mathrm{E}+00$ \\
\hline \multicolumn{9}{|l|}{ Phosphorus Pentoxide } \\
\hline PYRENE & 1.982E-02 & & $2.363 E-02$ & $5.929 E-03$ & $1.770 E-02$ & $5.607 E-03$ & $3.225 E-04$ & $4.085 E+00$ \\
\hline TOLUENE & $8.164 E-03$ & & $3.759 E+00$ & $3.732 E+00$ & $2.736 E-02$ & $3.732 E+00$ & $2.206 E-05$ & 4.619E-01 \\
\hline \multicolumn{9}{|l|}{ Zinc Dichloride Pentoxide } \\
\hline \multicolumn{9}{|l|}{ Zinc Oxalate } \\
\hline \multicolumn{9}{|l|}{ Zinc Hydroxide } \\
\hline \multicolumn{9}{|l|}{ Zirconium EDTA } \\
\hline \multicolumn{9}{|l|}{ Zirconium Oxide } \\
\hline Total $\mathrm{g} / \mathrm{hr}$ & $4.870 \mathrm{E}+04$ & $4.536 \mathrm{E}+04$ & $5.958 \mathrm{E}+04$ & $1.238 \mathrm{E}+04$ & $4.720 \mathrm{E}+04$ & $1.235 \mathrm{E}+04$ & $3.004 \mathrm{E}+01$ & $5.882 E+06$ \\
\hline Volume, L/hr & $4.909 \mathrm{E}+01$ & $4.866 \mathrm{E}+01$ & $2.700 \mathrm{E}+04$ & $1.431 \mathrm{E}+04$ & $4.758 \mathrm{E}+01$ & $1.134 \mathrm{E}+04$ & $3.010 \mathrm{E}-02$ & $5.901 \mathrm{E}+03$ \\
\hline Enthalpy, cal/hr & $-1.839 E+08$ & $-1.671 \mathrm{E}+08$ & $-1.753 \mathrm{E}+08$ & $-2.325 \mathrm{E}+06$ & $-1.783 \mathrm{E}+08$ & $-2.300 \mathrm{E}+06$ & $-1.127 \mathrm{E}+05$ & $-2.231 \mathrm{E}+10$ \\
\hline Vapor fraction & & & $2.575 \mathrm{E}-01$ & $1.000 \mathrm{E}+00$ & & $9.846 \mathrm{E}-01$ & & \\
\hline Solid fraction & 8.066E-06 & & & & $4.628 \mathrm{E}-06$ & & $6.064 \mathrm{E}-04$ & $1.008 \mathrm{E}-06$ \\
\hline \multicolumn{9}{|l|}{ Redox Pot, volts } \\
\hline lonic Strength & $7.692 \mathrm{E}-07$ & $1.250 \mathrm{E}-06$ & 9.197E-07 & & $1.412 \mathrm{E}-06$ & $1.584 \mathrm{E}-06$ & $1.584 \mathrm{E}-06$ & $1.491 \mathrm{E}-07$ \\
\hline
\end{tabular}




\subsection{APPENDIX B - DETAILED DESCRIPTION OF WASTE FEED EVAPORATOR OLI MODEL}

This appendix describes in detail the 5-stage waste feed evaporator (FEP) OLI model used in the offgas regulatory modeling described in the main body of this report. The FEP model was used to generate the total recycle stream properties like flow rate and composition that were then used in the offgas regulatory runs described in the main body of this report. Figure B-1 shows an overview of the FEP OLI model with the Evaporator, Ultrafiltration Phase $1 \& 2$, and First \& Second Caustic Washes, and Total Recycle. Note that the FEP model was driven by the density of the UF1 filtrate stream. Figure B-2 shows the first part of the Waste Feed Evaporator OLI Model: the Evaporator, Ultrafiltration Phase 1, and First \& Second Caustic Wash Splits. Figure B-3 shows the second part of the Waste Feed Evaporator OLI Model: First Caustic Wash. The first caustic wash is broken into five phases FCW1FCW5 to simulate multiple volume transfers. Only the first two caustic washes are shown. The other caustic washes are identical in the first caustic wash. Figure B-4 shows the last part of the Waste Feed Evaporator OLI Model: Leach, Ultrafiltration Phase 2, Caustic and Acid Rinse, Total Recycle, and Second Caustic Wash. Note that the second caustic wash is broken into five phases SCW1-SCW5 to simulate multiple volume transfers. Only the first phase is shown as the others are identical.

Table B- 1 shows the output of the FEP OLI model run for UF1 filtrate density of $1.22 \mathrm{~g} / \mathrm{ml}$. Table B- 2 shows the output from the FEP model run for UF1 filtrate density of $1.35 \mathrm{~g} / \mathrm{ml}$. Each of these runs took about 8 hours to converge on a $1.8 \mathrm{GHz}$ PC. The lengthy run time was due to the large number of species (about 395) plus the

density feedback control loop. Run time was significantly reduced by dropping the species $\mathrm{CrOH} 3$ or chromium hydroxide from the HLW Offgas Condensate (SBS Recycle) stream. In some cases the presence of $\mathrm{Cr}(\mathrm{OH}) 3$ would cause the OLI model not to converge. 
WSRC-TR-2002-00550, Rev. 1

SRT-RPP-2002-0027

Figure B-1. Waste Feed Evaporator Model Overview: Evaporator, Ultrafiltration Phase 1 \& 2, and First \& Second Caustic Washes, and Total Recycle

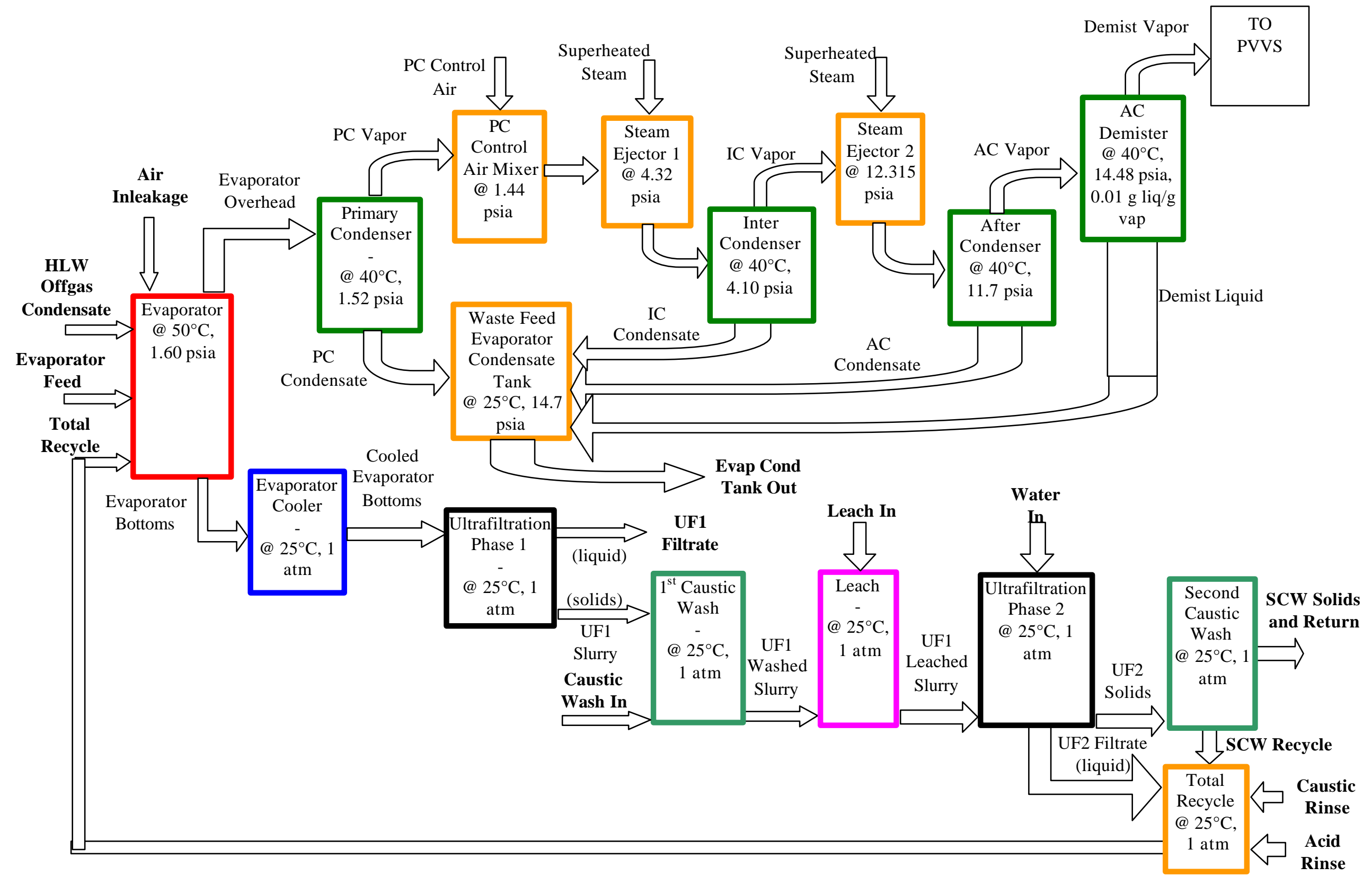


Figure B-2. Waste Feed Evaporator OLI Model Diagram - Evaporator, Ultrafiltration Phase 1, and First \& Second Caustic Wash Splits

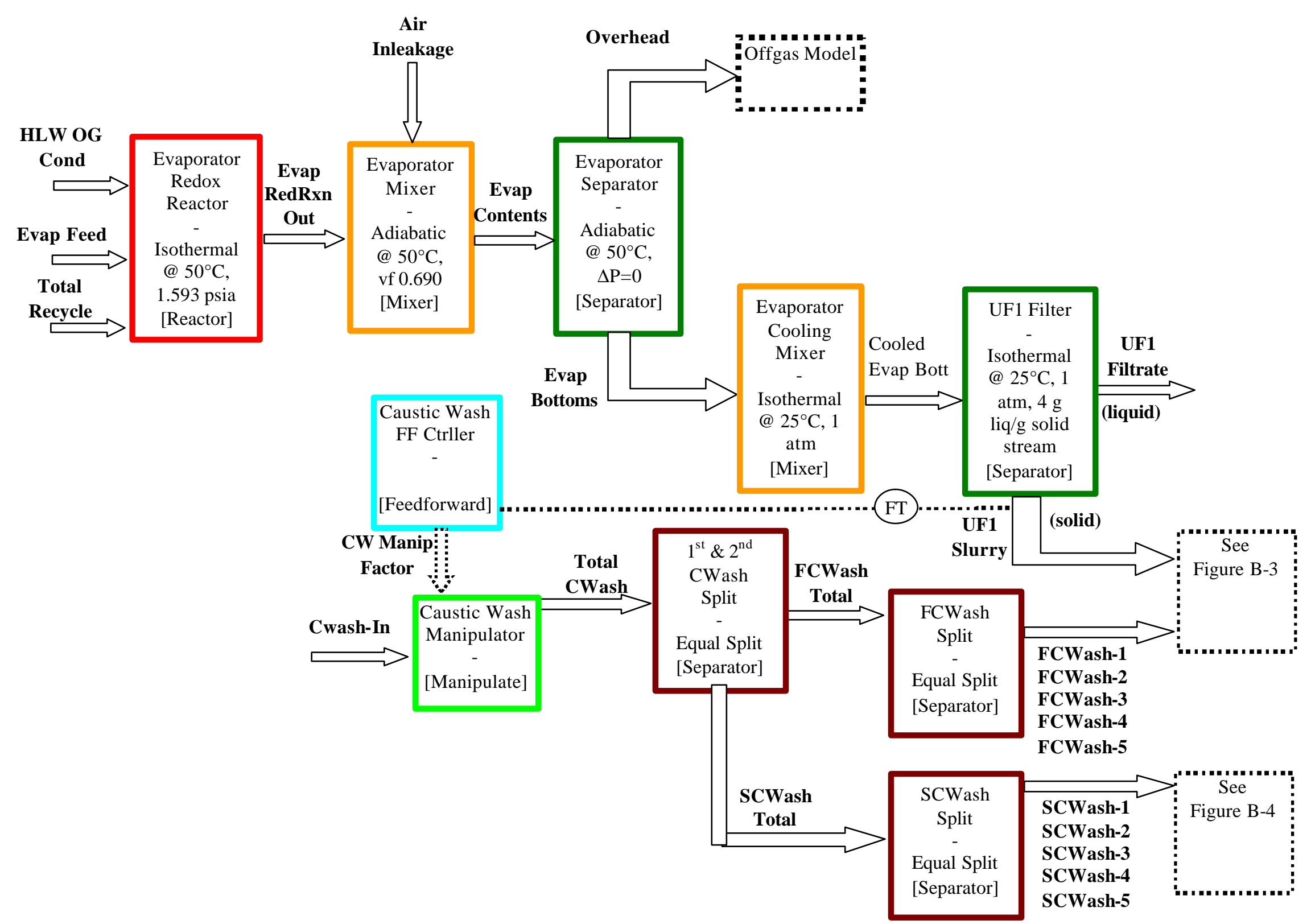


WSRC-TR-2002-00550, Rev. 1

SRT-RPP-2002-0027

Figure B-3. Waste Feed Evaporator OLI Model Diagram - First Caustic Wash (Broken into 5 phases FCW1-FCW5 to simulate multiple volume transfers)

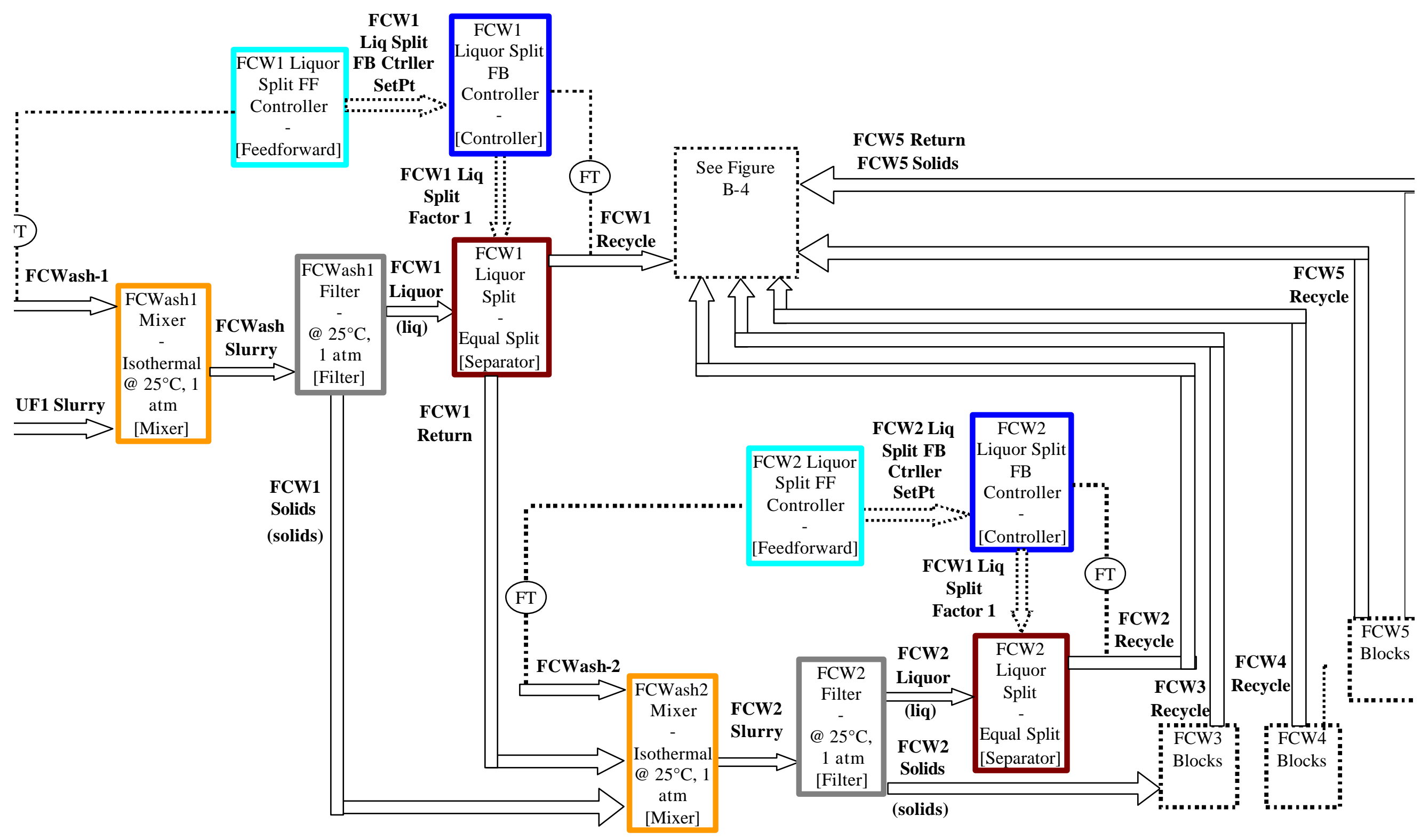




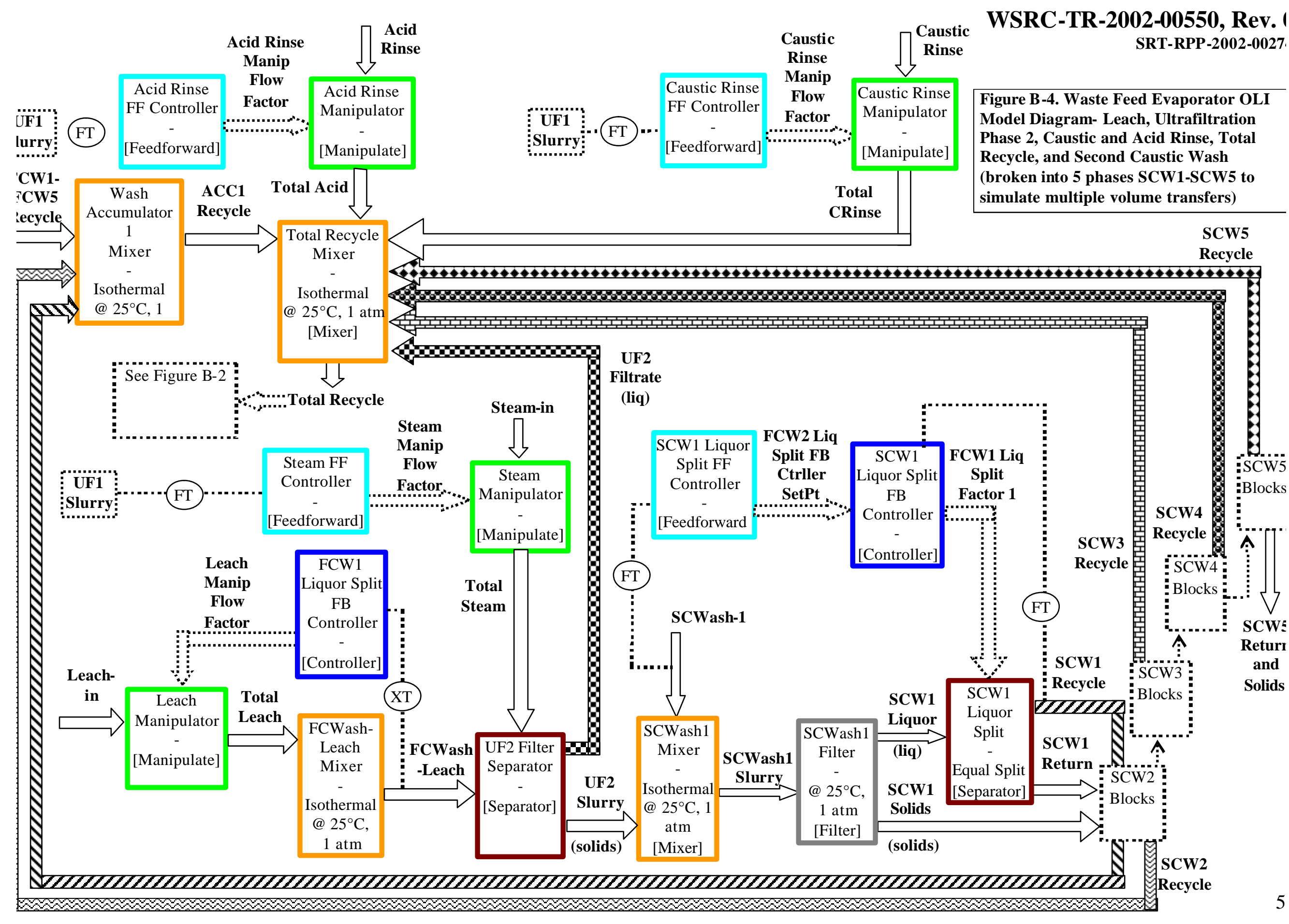


Table B - 1. Waste Feed Evaporator 5 Stage Model Results for UF 1.22 Density Case

\begin{tabular}{|c|c|c|c|c|c|c|c|c|}
\hline Stream & Air Inleakage & $\begin{array}{c}\text { EVAP } \\
\text { REDRXN } \\
\text { OUT }\end{array}$ & $\begin{array}{c}\text { Evap } \\
\text { Contents }\end{array}$ & Overhead & $\begin{array}{c}\text { Evap } \\
\text { Bottoms }\end{array}$ & $\begin{array}{c}\text { Cooled Evap } \\
\text { Bott }\end{array}$ & UF1 Filtrate & UF1 Slurry \\
\hline Phase & Vapor & Mixed & Mixed & Vapor & Mixed & Mixed & Aqueous & Mixed \\
\hline Temperature, $\mathrm{C}$ & 25 & 50 & 50 & 50 & 50 & 25 & 25 & 25 \\
\hline Pressure, atm & 1 & 0.108335 & 0.108583 & 0.108583 & 0.108583 & 1 & 1 & 1 \\
\hline $\mathrm{pH}$ & & 12.6538 & 12.6388 & & 12.6388 & 13.5083 & 13.5083 & 13.5083 \\
\hline Total mol/hr & 135.18 & 390030 & 390165 & 268643 & 121522 & 121511 & 118779 & 2732.49 \\
\hline Flow Units & $\mathrm{g} / \mathrm{hr}$ & $\mathrm{g} / \mathrm{hr}$ & $\mathrm{g} / \mathrm{hr}$ & $\mathrm{g} / \mathrm{hr}$ & $\mathrm{g} / \mathrm{hr}$ & $\mathrm{g} / \mathrm{hr}$ & $\mathrm{g} / \mathrm{hr}$ & $\mathrm{g} / \mathrm{hr}$ \\
\hline Acetic Acid, Dimer & & $1.430 \mathrm{E}-18$ & $1.440 \mathrm{E}-18$ & $1.440 \mathrm{E}-18$ & & & & \\
\hline Acetic Acid & & $2.647 \mathrm{E}-06$ & $2.643 \mathrm{E}-06$ & $2.643 \mathrm{E}-06$ & & & & \\
\hline Aluminum Oxide & & $4.810 \mathrm{E}+03$ & $4.810 \mathrm{E}+03$ & & $4.810 \mathrm{E}+03$ & $4.243 \mathrm{E}+03$ & $4.150 \mathrm{E}+03$ & $9.202 \mathrm{E}+01$ \\
\hline Aldrin & & $4.195 E+00$ & $4.195 E+00$ & $4.171 E+00$ & $2.408 E-02$ & $2.408 E-02$ & $2.355 E-02$ & $5.222 E-04$ \\
\hline Aluminum Hydrogen EDTA & & $1.190 \mathrm{E}+03$ & $1.190 \mathrm{E}+03$ & & $1.190 \mathrm{E}+03$ & $1.429 \mathrm{E}+03$ & $1.398 \mathrm{E}+03$ & $3.100 \mathrm{E}+01$ \\
\hline Aluminum Hydroxide & & & & & & $7.799 \mathrm{E}+02$ & & $7.799 \mathrm{E}+02$ \\
\hline Benzene & & $4.194 E+00$ & $4.194 E+00$ & $4.193 E+00$ & $1.188 E-04$ & $1.188 E-04$ & $1.162 E-04$ & $2.577 E-06$ \\
\hline $\begin{array}{c}\text { Bis(2-ethylhexyl)phthalate } \\
\text { (BEHP) }\end{array}$ & & $4.195 E+00$ & $4.195 E+00$ & $3.893 E+00$ & $3.026 E-01$ & $3.026 E-01$ & $2.960 E-01$ & $6.563 E-03$ \\
\hline Benzo(a)pyrene (BaP) & & $4.181 E+00$ & $4.181 E+00$ & & $4.181 E+00$ & $4.181 E+00$ & $1.923 E-03$ & $4.180 E+00$ \\
\hline Phenol & & $4.223 E+00$ & $4.223 E+00$ & $6.495 E-03$ & $4.217 E+00$ & $4.217 E+00$ & $4.125 E+00$ & $9.146 E-02$ \\
\hline Calcium Chloride Oxide & & & & & & & & \\
\hline Calcium Oxalate & & $2.279 \mathrm{E}+02$ & $2.251 \mathrm{E}+02$ & & $2.251 \mathrm{E}+02$ & $4.127 \mathrm{E}+02$ & $4.037 \mathrm{E}+02$ & $8.951 \mathrm{E}+00$ \\
\hline \multicolumn{9}{|l|}{ Calcium Oxalate Monohydrate } \\
\hline \multicolumn{9}{|l|}{ Calcium Carbonate } \\
\hline \multicolumn{9}{|l|}{ Calcium Dichromate (VI) } \\
\hline Calcium Fluoride & & $1.649 \mathrm{E}+03$ & $1.651 \mathrm{E}+03$ & & $1.651 \mathrm{E}+03$ & $1.536 \mathrm{E}+03$ & & $1.536 \mathrm{E}+03$ \\
\hline \multicolumn{9}{|l|}{ Calcium Hydroxide } \\
\hline Pentachlorophenol & & $4.199 E+00$ & $4.199 E+00$ & $3.835 E+00$ & $3.641 E-01$ & $3.641 E-01$ & $3.562 E-01$ & $7.898 E-03$ \\
\hline Hexachlorobenzene & & $4.188 E+00$ & $4.188 E+00$ & & $4.188 E+00$ & $4.188 E+00$ & $4.591 E-03$ & $4.183 E+00$ \\
\hline Chlorobenzene & & $4.190 E+00$ & $4.190 E+00$ & $4.190 E+00$ & $2.548 E-04$ & $2.548 E-04$ & $2.492 E-04$ & $5.526 E-06$ \\
\hline 2-Chloronaphthalene & & $4.194 E+00$ & $4.194 E+00$ & $4.194 E+00$ & $6.844 E-04$ & $6.844 E-04$ & $6.696 E-04$ & $1.485 E-05$ \\
\hline Carbon Dioxide & $1.949 \mathrm{E}+00$ & $7.899 \mathrm{E}+04$ & $7.899 \mathrm{E}+04$ & $2.500 \mathrm{E}-01$ & $7.899 \mathrm{E}+04$ & $7.899 \mathrm{E}+04$ & $7.727 \mathrm{E}+04$ & $1.713 \mathrm{E}+03$ \\
\hline Cesium Acetate & & $1.985 \mathrm{E}+02$ & $1.985 \mathrm{E}+02$ & & $1.985 \mathrm{E}+02$ & $1.985 \mathrm{E}+02$ & $1.942 \mathrm{E}+02$ & $4.306 \mathrm{E}+00$ \\
\hline \multicolumn{9}{|l|}{ Cesium Glycolate } \\
\hline Dibenz[a,h]anthracene & & $4.192 E+00$ & $4.192 E+00$ & $4.023 E+00$ & $1.691 E-01$ & $1.691 E-01$ & $1.655 E-01$ & $3.669 E-03$ \\
\hline 1,2-dibromoethane & & $2.806 E+01$ & $2.806 E+01$ & $2.806 E+01$ & $6.123 E-03$ & $6.123 E-03$ & $5.990 E-03$ & $1.328 E-04$ \\
\hline Diethyl phthalate & & $4.208 E+00$ & $4.208 E+00$ & $3.288 E+00$ & $9.198 E-01$ & $9.198 E-01$ & $8.998 E-01$ & $1.995 E-02$ \\
\hline \multicolumn{9}{|l|}{ Iron (III) Oxalate } \\
\hline \multicolumn{9}{|l|}{ Iron (III) Citrate } \\
\hline Iron (III) Hydrogen EDTA & & $6.074 \mathrm{E}+02$ & $6.074 \mathrm{E}+02$ & & $6.074 \mathrm{E}+02$ & $3.388 \mathrm{E}+02$ & $3.314 \mathrm{E}+02$ & $7.349 \mathrm{E}+00$ \\
\hline Iron (III) Hydroxide & & & & & & $8.318 \mathrm{E}+01$ & & $8.318 \mathrm{E}+01$ \\
\hline Formic Acid, dimer & & $7.040 \mathrm{E}-25$ & $7.088 \mathrm{E}-25$ & $7.088 \mathrm{E}-25$ & & & & \\
\hline Hydrofluoride, dimer & & $2.008 \mathrm{E}-22$ & $2.039 \mathrm{E}-22$ & $2.039 \mathrm{E}-22$ & & & & \\
\hline 2,2'-Iminobisacetic acid & & $1.140 \mathrm{E}+03$ & $1.140 \mathrm{E}+03$ & & $1.140 \mathrm{E}+03$ & $1.140 \mathrm{E}+03$ & $1.115 \mathrm{E}+03$ & $2.472 \mathrm{E}+01$ \\
\hline Water & $1.819 \mathrm{E}+01$ & $6.727 \mathrm{E}+06$ & $6.727 \mathrm{E}+06$ & $4.837 \mathrm{E}+06$ & $1.890 \mathrm{E}+06$ & $1.891 \mathrm{E}+06$ & $1.850 \mathrm{E}+06$ & $4.102 \mathrm{E}+04$ \\
\hline \multicolumn{9}{|l|}{ Sulfuric Acid } \\
\hline Hydrogen Chloride & & $1.035 \mathrm{E}+04$ & $1.035 \mathrm{E}+04$ & $4.604 \mathrm{E}-11$ & $1.035 \mathrm{E}+04$ & $1.035 \mathrm{E}+04$ & $1.012 \mathrm{E}+04$ & $2.244 \mathrm{E}+02$ \\
\hline Formic Acid & & $3.987 \mathrm{E}+02$ & $3.987 \mathrm{E}+02$ & $2.927 \mathrm{E}-07$ & $3.987 \mathrm{E}+02$ & $3.987 \mathrm{E}+02$ & $3.900 \mathrm{E}+02$ & $8.647 \mathrm{E}+00$ \\
\hline Hydrofluoric Acid & & $3.020 \mathrm{E}+03$ & $3.019 \mathrm{E}+03$ & $1.686 \mathrm{E}-07$ & $3.019 \mathrm{E}+03$ & $3.077 \mathrm{E}+03$ & $3.011 \mathrm{E}+03$ & $6.675 \mathrm{E}+01$ \\
\hline Mercury (II) Chloride & & $2.220 E-07$ & $2.240 E-07$ & $2.240 E-07$ & & & & \\
\hline Mercury (elemental) & & $6.349 E+01$ & $6.349 E+01$ & $6.349 E+01$ & $5.835 E-04$ & $5.835 E-04$ & $5.708 E-04$ & $1.266 E-05$ \\
\hline Mercury (II) Oxide & & $6.850 E+01$ & $6.850 E+01$ & & $6.850 E+01$ & $6.850 E+01$ & $3.899 E+01$ & $2.951 E+01$ \\
\hline Nitrous (III) Acid & & $9.748 \mathrm{E}+04$ & $9.766 \mathrm{E}+04$ & $8.501 \mathrm{E}-03$ & $9.766 \mathrm{E}+04$ & $9.341 \mathrm{E}+04$ & $9.139 \mathrm{E}+04$ & $2.026 \mathrm{E}+03$ \\
\hline Nitric Acid & & $6.417 \mathrm{E}+04$ & $6.417 \mathrm{E}+04$ & $1.712 \mathrm{E}-11$ & $6.417 \mathrm{E}+04$ & $6.417 \mathrm{E}+04$ & $6.278 \mathrm{E}+04$ & $1.392 \mathrm{E}+03$ \\
\hline Hexachlorobutadiene & & $4.200 E+00$ & $4.200 E+00$ & $4.200 E+00$ & $5.341 E-06$ & $5.341 E-06$ & $5.225 E-06$ & $1.158 E-07$ \\
\hline Potassium Citrate & & $2.842 \mathrm{E}+03$ & $2.842 \mathrm{E}+03$ & & $2.842 \mathrm{E}+03$ & $2.842 \mathrm{E}+03$ & $2.780 \mathrm{E}+03$ & $6.164 \mathrm{E}+01$ \\
\hline
\end{tabular}


WSRC-TR-2002-00550, Rev. 1 SRT-RPP-2002-0027.

\begin{tabular}{|c|c|c|c|c|c|c|c|c|}
\hline Stream & Air Inleakage & $\begin{array}{c}\text { EVAP } \\
\text { REDRXN } \\
\text { OUT }\end{array}$ & $\begin{array}{c}\text { Evap } \\
\text { Contents }\end{array}$ & Overhead & $\begin{array}{c}\text { Evap } \\
\text { Bottoms }\end{array}$ & $\begin{array}{c}\text { Cooled Evap } \\
\text { Bott }\end{array}$ & UF1 Filtrate & UF1 Slurry \\
\hline \multicolumn{9}{|l|}{ Potassium Acetate } \\
\hline Potassium Chloride & & $3.760 \mathrm{E}+04$ & $3.760 \mathrm{E}+04$ & & $3.760 \mathrm{E}+04$ & $3.760 \mathrm{E}+04$ & $3.678 \mathrm{E}+04$ & $8.155 \mathrm{E}+02$ \\
\hline \multicolumn{9}{|l|}{ Potassium Glycolate } \\
\hline \multicolumn{9}{|l|}{ Potassium Nitrate (VI) } \\
\hline gamma-BHC (Lindane) & & $4.209 E+00$ & $4.209 E+00$ & $4.194 E+00$ & $1.487 E-02$ & $1.487 E-02$ & $1.455 E-02$ & $3.226 E-04$ \\
\hline $\begin{array}{c}\text { 4-methyl-2-pentanone } \\
(\text { (MIBK) }\end{array}$ & & $4.197 E+00$ & $4.197 E+00$ & $4.194 E+00$ & $2.840 E-03$ & $2.840 E-03$ & $2.778 E-03$ & $6.160 E-05$ \\
\hline Magnesium Oxalate & & $2.879 \mathrm{E}-02$ & $2.947 \mathrm{E}-02$ & & $2.947 \mathrm{E}-02$ & $1.594 \mathrm{E}-02$ & $1.559 \mathrm{E}-02$ & $3.457 \mathrm{E}-04$ \\
\hline \multicolumn{9}{|l|}{ Magnesium Dichromate (VI) } \\
\hline Magnesium Hydroxide & & $2.385 \mathrm{E}+02$ & $2.385 \mathrm{E}+02$ & & $2.385 \mathrm{E}+02$ & $2.385 \mathrm{E}+02$ & & $2.385 \mathrm{E}+02$ \\
\hline \multicolumn{9}{|l|}{$\begin{array}{l}\text { Manganese (II) } \\
\text { Orthophosphate }\end{array}$} \\
\hline Manganese (II) Oxalate & & $1.319 \mathrm{E}+00$ & $1.319 \mathrm{E}+00$ & & $1.319 \mathrm{E}+00$ & $1.319 \mathrm{E}+00$ & $1.290 \mathrm{E}+00$ & $2.860 \mathrm{E}-02$ \\
\hline \multicolumn{9}{|l|}{ Manganese (II) Hydroxide } \\
\hline Nitrogen & $2.970 \mathrm{E}+03$ & $3.815 \mathrm{E}-06$ & $2.970 \mathrm{E}+03$ & $2.970 \mathrm{E}+03$ & $5.856 \mathrm{E}-04$ & $5.856 \mathrm{E}-04$ & $5.729 \mathrm{E}-04$ & $1.270 \mathrm{E}-05$ \\
\hline Sodium Oxalate & & $1.381 \mathrm{E}+04$ & $1.382 \mathrm{E}+04$ & & $1.382 \mathrm{E}+04$ & $1.362 \mathrm{E}+04$ & $5.317 \mathrm{E}+03$ & $8.304 \mathrm{E}+03$ \\
\hline Sodium Chromate (VI) & & $8.404 \mathrm{E}+03$ & $8.404 \mathrm{E}+03$ & & $8.404 \mathrm{E}+03$ & $8.404 \mathrm{E}+03$ & $8.222 \mathrm{E}+03$ & $1.823 \mathrm{E}+02$ \\
\hline \multicolumn{9}{|l|}{ Sodium Fluoride Sulfate } \\
\hline Sodium Acetate & & $1.847 \mathrm{E}+02$ & $1.847 \mathrm{E}+02$ & & $1.847 \mathrm{E}+02$ & $1.847 \mathrm{E}+02$ & $1.807 \mathrm{E}+02$ & $4.007 \mathrm{E}+00$ \\
\hline Sodium Glycolate & & $4.586 \mathrm{E}+03$ & $4.586 \mathrm{E}+03$ & & $4.586 \mathrm{E}+03$ & $4.586 \mathrm{E}+03$ & $4.487 \mathrm{E}+03$ & $9.948 \mathrm{E}+01$ \\
\hline Sodium Nitrite & & $6.731 \mathrm{E}+04$ & $6.704 \mathrm{E}+04$ & & $6.704 \mathrm{E}+04$ & $7.327 \mathrm{E}+04$ & $7.168 \mathrm{E}+04$ & $1.589 \mathrm{E}+03$ \\
\hline Sodium Hydroxide & & $3.554 \mathrm{E}+05$ & $3.555 \mathrm{E}+05$ & & $3.555 \mathrm{E}+05$ & $3.520 \mathrm{E}+05$ & $3.444 \mathrm{E}+05$ & $7.636 \mathrm{E}+03$ \\
\hline Naphthalene & & $4.188 \mathrm{E}+00$ & $4.188 \mathrm{E}+00$ & $4.188 \mathrm{E}+00$ & $7.531 \mathrm{E}-04$ & $7.531 \mathrm{E}-04$ & $7.368 \mathrm{E}-04$ & $1.634 \mathrm{E}-05$ \\
\hline Sodium Aluminosilicate Gel & & $3.617 \mathrm{E}+03$ & $3.618 \mathrm{E}+03$ & & $3.618 \mathrm{E}+03$ & $3.690 \mathrm{E}+03$ & & $3.690 \mathrm{E}+03$ \\
\hline \multicolumn{9}{|l|}{ Nickel Orthophosphate } \\
\hline Nickel (II) Oxalate & & $3.976 \mathrm{E}+00$ & $3.976 \mathrm{E}+00$ & & $3.977 \mathrm{E}+00$ & $3.976 \mathrm{E}+00$ & $3.890 \mathrm{E}+00$ & $8.625 \mathrm{E}-02$ \\
\hline \multicolumn{9}{|l|}{ Nickel (II) Hydroxide } \\
\hline Oxygen & $8.994 \mathrm{E}+02$ & $2.122 \mathrm{E}-06$ & $8.994 \mathrm{E}+02$ & $8.994 \mathrm{E}+02$ & $3.258 \mathrm{E}-04$ & $3.258 \mathrm{E}-04$ & $3.187 \mathrm{E}-04$ & 7.067E-06 \\
\hline Phosphorus Pentoxide & & $6.433 \mathrm{E}+02$ & $6.433 \mathrm{E}+02$ & & $6.433 \mathrm{E}+02$ & $6.433 \mathrm{E}+02$ & $6.294 \mathrm{E}+02$ & $1.395 \mathrm{E}+01$ \\
\hline PYRENE & & $4.202 \mathrm{E}+00$ & $4.202 \mathrm{E}+00$ & $4.178 \mathrm{E}+00$ & $2.339 \mathrm{E}-02$ & $2.339 \mathrm{E}-02$ & $2.288 \mathrm{E}-02$ & $5.073 \mathrm{E}-04$ \\
\hline Silicon Dioxide & & $1.142 \mathrm{E}+02$ & $1.141 \mathrm{E}+02$ & & $1.141 \mathrm{E}+02$ & $8.786 \mathrm{E}+01$ & $8.596 \mathrm{E}+01$ & $1.906 \mathrm{E}+00$ \\
\hline Sulfur Trioxide & & $4.780 \mathrm{E}+04$ & $4.780 \mathrm{E}+04$ & & $4.780 \mathrm{E}+04$ & $4.780 \mathrm{E}+04$ & $4.676 \mathrm{E}+04$ & $1.037 \mathrm{E}+03$ \\
\hline 1,2,4-trichlorobenzene & & $4.193 \mathrm{E}+00$ & $4.193 \mathrm{E}+00$ & $4.193 \mathrm{E}+00$ & $1.590 \mathrm{E}-04$ & $1.590 \mathrm{E}-04$ & $1.556 \mathrm{E}-04$ & $3.449 \mathrm{E}-06$ \\
\hline 1,2,3-trichloropropa ne & & $4.198 \mathrm{E}+00$ & $4.198 \mathrm{E}+00$ & $4.197 \mathrm{E}+00$ & $1.121 \mathrm{E}-03$ & $1.121 \mathrm{E}-03$ & $1.096 \mathrm{E}-03$ & $2.431 \mathrm{E}-05$ \\
\hline TOLUENE & & $4.194 \mathrm{E}+00$ & $4.194 \mathrm{E}+00$ & $4.194 \mathrm{E}+00$ & $9.874 \mathrm{E}-05$ & $9.874 \mathrm{E}-05$ & $9.660 \mathrm{E}-05$ & $2.142 \mathrm{E}-06$ \\
\hline \multicolumn{9}{|l|}{ Zinc Dichloride Pentoxide } \\
\hline Zinc Oxalate & & $1.147 \mathrm{E}+03$ & $1.147 \mathrm{E}+03$ & & $1.147 \mathrm{E}+03$ & $1.147 \mathrm{E}+03$ & $1.122 \mathrm{E}+03$ & $2.487 \mathrm{E}+01$ \\
\hline \multicolumn{9}{|l|}{ Zinc Hydroxide } \\
\hline Zirconium EDTA & & $6.821 \mathrm{E}+00$ & $6.806 \mathrm{E}+00$ & & $6.806 \mathrm{E}+00$ & $1.525 \mathrm{E}+01$ & $1.492 \mathrm{E}+01$ & $3.308 \mathrm{E}-01$ \\
\hline Zirconium Oxide & & $2.737 \mathrm{E}+00$ & $2.742 \mathrm{E}+00$ & & $2.742 \mathrm{E}+00$ & & & \\
\hline Total $\mathrm{g} / \mathrm{hr}$ & $3.889 \mathrm{E}+03$ & $7.535 \mathrm{E}+06$ & $7.539 \mathrm{E}+06$ & $4.841 \mathrm{E}+06$ & $2.698 \mathrm{E}+06$ & $2.698 \mathrm{E}+06$ & $2.625 \mathrm{E}+06$ & $7.276 \mathrm{E}+04$ \\
\hline Volume, L/hr & $3.307 \mathrm{E}+03$ & $6.617 \mathrm{E}+07$ & $6.552 \mathrm{E}+07$ & $6.552 \mathrm{E}+07$ & $2.252 \mathrm{E}+03$ & $2.203 \mathrm{E}+03$ & $2.152 \mathrm{E}+03$ & $5.145 \mathrm{E}+01$ \\
\hline Enthalpy, cal/hr & $-6.275 \mathrm{E}+04$ & $-2.430 \mathrm{E}+10$ & $-2.432 \mathrm{E}+10$ & $-1.547 \mathrm{E}+10$ & $-8.855 \mathrm{E}+09$ & $-8.904 \mathrm{E}+09$ & $-8.669 \mathrm{E}+09$ & $-2.350 \mathrm{E}+08$ \\
\hline Vapor fraction & $1.000 \mathrm{E}+00$ & $6.940 \mathrm{E}-01$ & $6.885 \mathrm{E}-01$ & $1.000 \mathrm{E}+00$ & & & & \\
\hline Solid fraction & & $1.381 \mathrm{E}-04$ & $1.316 \mathrm{E}-04$ & & $4.226 \mathrm{E}-04$ & $8.146 \mathrm{E}-04$ & & $3.622 \mathrm{E}-02$ \\
\hline \multicolumn{9}{|l|}{ Organic fraction } \\
\hline Osmotic Pres, atm & & $1.802 \mathrm{E}+02$ & $1.774 \mathrm{E}+02$ & & $1.774 \mathrm{E}+02$ & $1.739 \mathrm{E}+02$ & $1.739 \mathrm{E}+02$ & $1.739 \mathrm{E}+02$ \\
\hline \multicolumn{9}{|l|}{ Redox Pot, volts } \\
\hline E-Con, 1/ohm-cm & & $3.040 \mathrm{E}-01$ & $1.860 \mathrm{E}-01$ & & $1.860 \mathrm{E}-01$ & $2.012 \mathrm{E}-01$ & & \\
\hline E-Con, cm2/ohm-mol & & $4.044 \mathrm{E}+01$ & $2.497 \mathrm{E}+01$ & & $2.497 \mathrm{E}+01$ & $2.663 \mathrm{E}+01$ & & \\
\hline Abs Visc, cP & & $1.444 \mathrm{E}+00$ & $1.421 \mathrm{E}+00$ & & $1.421 \mathrm{E}+00$ & $2.328 \mathrm{E}+00$ & & \\
\hline Rel Visc & & $2.639 \mathrm{E}+00$ & $2.597 \mathrm{E}+00$ & & $2.597 \mathrm{E}+00$ & $2.613 \mathrm{E}+00$ & & \\
\hline Ionic Strength & & $5.903 \mathrm{E}+00$ & $5.791 \mathrm{E}+00$ & & $5.791 \mathrm{E}+00$ & $5.571 \mathrm{E}+00$ & $5.571 \mathrm{E}+00$ & $5.571 \mathrm{E}+00$ \\
\hline
\end{tabular}


WSRC-TR-2002-00550, Rev. 1

SRT-RPP-2002-0027.

Table B- 1. Waste Feed Evaporator 5 Stage Model Results for UF 1.22 Density Case (cont'd) ${ }^{8}$

\begin{tabular}{|c|c|c|c|c|c|c|c|c|c|}
\hline Stream & CWash-In & $\begin{array}{c}\text { Total } \\
\text { CWash }\end{array}$ & $\begin{array}{c}\text { FCWash } \\
\text { Total }\end{array}$ & $\begin{array}{c}\text { SCWash } \\
\text { Total }\end{array}$ & FCWash-1 & FCWash-2 & FCWash-3 & FCWash-4 & FCWash-5 \\
\hline Phase & Aqueous & Aqueous & Aqueous & Aqueous & Aqueous & Aqueous & Aqueous & Aqueous & Aqueous \\
\hline Temperature, $\mathrm{C}$ & 25 & 25 & 25 & 25 & 25 & 25 & 25 & 25 & 25 \\
\hline Pressure, atm & 1 & 1 & 1 & 1 & 1 & 1 & 1 & 1 & 1 \\
\hline$\overline{\mathrm{pH}}$ & 11.9511 & 11.9511 & 11.9511 & 11.9511 & 11.9511 & 11.9511 & 11.9511 & 11.9511 & 11.9511 \\
\hline Total mol $/ \mathrm{hr}$ & 55.346 & 55.346 & 27.673 & 27.673 & 5.5346 & 5.5346 & 5.5346 & 5.5346 & 5.5346 \\
\hline Flow Units & $\mathrm{g} / \mathrm{hr}$ & $\mathrm{g} / \mathrm{hr}$ & $\mathrm{g} / \mathrm{hr}$ & $\mathrm{g} / \mathrm{hr}$ & $\mathrm{g} / \mathrm{hr}$ & $\mathrm{g} / \mathrm{hr}$ & $\mathrm{g} / \mathrm{hr}$ & $\mathrm{g} / \mathrm{hr}$ & $\mathrm{g} / \mathrm{hr}$ \\
\hline \\
\hline Acetic Acid & & & & & & & & & \\
\hline \multicolumn{10}{|l|}{ Aluminum Oxide } \\
\hline \multirow{2}{*}{\multicolumn{10}{|c|}{$\begin{array}{c}\text { Aldrin } \\
\text { Aluminum Hydrogen EDTA }\end{array}$}} \\
\hline \multirow{2}{*}{\multicolumn{10}{|c|}{ Aluminum Hydrogen EDTA }} \\
\hline \multirow{2}{*}{\multicolumn{7}{|c|}{$\begin{array}{c}\text { Aluminum Hydroxide } \\
\text { Benzene }\end{array}$}} & & & \\
\hline & & & & & & & & & \\
\hline \multicolumn{10}{|l|}{$\begin{array}{c}\text { Bis(2-ethylhexyl)phthalate } \\
\text { (BEHP) }\end{array}$} \\
\hline \multicolumn{10}{|l|}{ Benzo(a)pyrene (BaP) } \\
\hline \multicolumn{10}{|l|}{ Phenol } \\
\hline \multirow{2}{*}{\multicolumn{10}{|c|}{$\begin{array}{c}\text { Calcium Chloride Oxide } \\
\text { Calcium Oxalate }\end{array}$}} \\
\hline & & & & & & & & & \\
\hline \multicolumn{10}{|c|}{ Calcium Oxalate Monohydrate } \\
\hline \multicolumn{10}{|l|}{ Calcium Carbonate } \\
\hline \multicolumn{10}{|l|}{ Calcium Dichromate $(\mathrm{VI})$} \\
\hline \multicolumn{10}{|l|}{ Calcium Fluoride } \\
\hline Calcium Hydroxide & & & & & & & & & \\
\hline Pentachlorophenol & & & & & & & & & \\
\hline \multicolumn{10}{|l|}{ Hexachlorobenzene } \\
\hline \multicolumn{10}{|l|}{ Chlorobenzene } \\
\hline \multicolumn{10}{|l|}{ 2-Chloronaphthalene } \\
\hline Carbon Dioxide & & & & & & & & & \\
\hline Cesium Acetate & & & & & & & & & \\
\hline Cesium Glycolate & & & & & & & & & \\
\hline Dibenz[a,h]anthracene & & & & & & & & & \\
\hline 1,2-dibromoethane & & & & & & & & & \\
\hline Diethyl phthalate & & & & & & & & & \\
\hline Iron (III) Oxalate & & & & & & & & & \\
\hline Iron (III) Citrate & & & & & & & & & \\
\hline Iron (III) Hydrogen EDTA & & & & & & & & & \\
\hline Iron (III) Hydroxide & & & & & & & & & \\
\hline Formic Acid, dimer & & & & & & & & & \\
\hline Hydrofluoride, dimer & & & & & & & & & \\
\hline 2,2'-Iminobisacetic acid & & & & & & & & & \\
\hline Water & $9.969 \mathrm{E}+02$ & $9.969 \mathrm{E}+02$ & $4.984 \mathrm{E}+02$ & $4.984 \mathrm{E}+02$ & $9.969 \mathrm{E}+01$ & $9.969 \mathrm{E}+01$ & $9.969 \mathrm{E}+01$ & $9.969 \mathrm{E}+01$ & $9.969 \mathrm{E}+01$ \\
\hline Sulfuric Acid & & & & & & & & & \\
\hline Hydrogen Chloride & & & & & & & & & \\
\hline Formic Acid & & & & & & & & & \\
\hline Hydrofluoric Acid & & & & & & & & & \\
\hline Mercury (II) Chloride & & & & & & & & & \\
\hline Mercury (elemental) & & & & & & & & & \\
\hline Mercury (II) Oxide & & & & & & & & & \\
\hline Nitrous (III) Acid & & & & & & & & & \\
\hline Nitric Acid & & & & & & & & & \\
\hline Hexachlorobutadiene & & & & & & & & & \\
\hline Potassium Citrate & & & & & & & & & \\
\hline Potassium Acetate & & & & & & & & & \\
\hline Potassium Chloride & & & & & & & & & \\
\hline
\end{tabular}


WSRC-TR-2002-00550, Rev. 1

SRT-RPP-2002-0027.

\begin{tabular}{|c|c|c|c|c|c|c|c|c|c|}
\hline Stream & CWash-In & $\begin{array}{l}\text { Total } \\
\text { CWash }\end{array}$ & $\begin{array}{l}\text { FCWash } \\
\text { Total }\end{array}$ & $\begin{array}{l}\text { SCWash } \\
\text { Total }\end{array}$ & FCWash-1 & FCWash-2 & FCWash-3 & FCWash-4 & FCWash-5 \\
\hline \multicolumn{10}{|l|}{ Potassium Glycolate } \\
\hline \multicolumn{10}{|l|}{ Potassium Nitrate (VI) } \\
\hline \multicolumn{10}{|l|}{ gamma-BHC (Lindane) } \\
\hline \multicolumn{10}{|l|}{$\begin{array}{c}\text { 4-methyl-2-pentanone } \\
\text { (MIBK) }\end{array}$} \\
\hline \multirow{2}{*}{\multicolumn{10}{|c|}{$\begin{array}{c}\text { Magnesium Oxalate } \\
\text { Magnesium Dichromate (VI) }\end{array}$}} \\
\hline & & & & & & & & & \\
\hline \multicolumn{10}{|l|}{ Magnesium Hydroxide } \\
\hline \multirow{2}{*}{\multicolumn{10}{|c|}{$\begin{array}{l}\text { Manganese (II) } \\
\text { Orthophosphate }\end{array}$}} \\
\hline & & & & & & & & & \\
\hline \multicolumn{10}{|l|}{ Manganese (II) Oxalate } \\
\hline \multicolumn{10}{|l|}{$\begin{array}{c}\text { Manganese (II) Hydroxide } \\
\text { Nitrogen }\end{array}$} \\
\hline \multirow{2}{*}{\multicolumn{10}{|c|}{$\begin{array}{c}\text { Nitrogen } \\
\text { Sodium Oxalate }\end{array}$}} \\
\hline & & & & & & & & & \\
\hline \multicolumn{10}{|l|}{ Sodium Chromate (VI) } \\
\hline \multicolumn{10}{|l|}{ Sodium Fluoride Sulfate } \\
\hline \multicolumn{10}{|l|}{ Sodium Acetate } \\
\hline \multirow{2}{*}{\multicolumn{10}{|c|}{$\begin{array}{c}\text { Sodium Glycolate } \\
\text { Sodium Nitrite }\end{array}$}} \\
\hline & & & & & & & & & \\
\hline Sodium Hydroxide & 3.999E-01 & $3.999 \mathrm{E}-01$ & $1.999 \mathrm{E}-01$ & $1.999 \mathrm{E}-01$ & $3.999 \mathrm{E}-02$ & $3.999 \mathrm{E}-02$ & $3.999 \mathrm{E}-02$ & $3.999 \mathrm{E}-02$ & $3.999 \mathrm{E}-02$ \\
\hline \multicolumn{10}{|l|}{ Naphthalene } \\
\hline \multicolumn{10}{|l|}{ Sodium Aluminosilicate Gel } \\
\hline \multicolumn{10}{|l|}{ Nickel Orthophosphate } \\
\hline \multicolumn{10}{|l|}{ Nickel (II) Oxalate } \\
\hline \multirow{2}{*}{\multicolumn{10}{|c|}{$\begin{array}{c}\text { Nickel (II) Hydroxide } \\
\text { Oxygen }\end{array}$}} \\
\hline & & & & & & & & & \\
\hline Phosphorus Pentoxide & & & & & & & & & \\
\hline PYRENE & & & & & & & & & \\
\hline Silicon Dioxide & & & & & & & & & \\
\hline Sulfur Trioxide & & & & & & & & & \\
\hline 1,2,4-trichlorobenzene & & & & & & & & & \\
\hline 1,2,3-trichloropropane & & & & & & & & & \\
\hline TOLUENE & & & & & & & & & \\
\hline Zinc Dichloride Pentoxid & & & & & & & & & \\
\hline Zinc Oxalate & & & & & & & & & \\
\hline Zinc Hydroxide & & & & & & & & & \\
\hline Zirconium EDTA & & & & & & & & & \\
\hline Zirconium Oxide & & & & & & & & & \\
\hline Total $\mathrm{g} / \mathrm{hr}$ & $9.973 \mathrm{E}+02$ & $9.973 \mathrm{E}+02$ & $4.986 \mathrm{E}+02$ & $4.986 \mathrm{E}+02$ & $9.973 \mathrm{E}+01$ & $9.973 \mathrm{E}+01$ & $9.973 \mathrm{E}+01$ & $9.973 \mathrm{E}+01$ & $9.973 \mathrm{E}+01$ \\
\hline Volume, $\mathrm{L} / \mathrm{hr}$ & $1.000 \mathrm{E}+00$ & $1.000 \mathrm{E}+00$ & $5.000 \mathrm{E}-01$ & $5.000 \mathrm{E}-01$ & $1.000 \mathrm{E}-01$ & $1.000 \mathrm{E}-01$ & $1.000 \mathrm{E}-01$ & $1.000 \mathrm{E}-01$ & $1.000 \mathrm{E}-01$ \\
\hline Enthalpy, cal/hr & $-3.781 \mathrm{E}+06$ & $-3.781 \mathrm{E}+06$ & $-1.891 \mathrm{E}+06$ & $-1.891 \mathrm{E}+06$ & $-3.781 \mathrm{E}+05$ & $-3.781 \mathrm{E}+05$ & $-3.781 \mathrm{E}+05$ & $-3.781 \mathrm{E}+05$ & $-3.781 \mathrm{E}+05$ \\
\hline Vapor fraction & & & & & & & & & \\
\hline Solid fraction & & & & & & & & & \\
\hline Organic fraction & & & & & & & & & \\
\hline Osmotic Pres, atm & $4.741 \mathrm{E}-01$ & 4.741E-01 & 4.741E-01 & $4.741 \mathrm{E}-01$ & $4.741 \mathrm{E}-01$ & 4.741E-01 & $4.741 \mathrm{E}-01$ & $4.741 \mathrm{E}-01$ & $4.741 \mathrm{E}-01$ \\
\hline Redox Pot, volts & & & & & & & & & \\
\hline E-Con, 1/ohm-cm & $2.366 \mathrm{E}-03$ & $2.366 \mathrm{E}-03$ & $2.366 \mathrm{E}-03$ & $2.366 \mathrm{E}-03$ & $2.366 \mathrm{E}-03$ & $2.366 \mathrm{E}-03$ & $2.366 \mathrm{E}-03$ & $2.366 \mathrm{E}-03$ & $2.366 \mathrm{E}-03$ \\
\hline E-Con, cm2/ohm-mol & $2.367 \mathrm{E}+02$ & $2.367 \mathrm{E}+02$ & $2.367 \mathrm{E}+02$ & $2.367 \mathrm{E}+02$ & $2.367 \mathrm{E}+02$ & $2.367 \mathrm{E}+02$ & $2.367 \mathrm{E}+02$ & $2.367 \mathrm{E}+02$ & $2.367 \mathrm{E}+02$ \\
\hline Abs Visc, cP & $8.929 \mathrm{E}-01$ & $8.929 \mathrm{E}-01$ & $8.929 \mathrm{E}-01$ & $8.929 \mathrm{E}-01$ & $8.929 \mathrm{E}-01$ & $8.929 \mathrm{E}-01$ & $8.929 \mathrm{E}-01$ & $8.929 \mathrm{E}-01$ & $8.929 \mathrm{E}-01$ \\
\hline Rel Visc & $1.002 \mathrm{E}+00$ & $1.002 \mathrm{E}+00$ & $1.002 \mathrm{E}+00$ & $1.002 \mathrm{E}+00$ & $1.002 \mathrm{E}+00$ & $1.002 \mathrm{E}+00$ & $1.002 \mathrm{E}+00$ & $1.002 \mathrm{E}+00$ & $1.002 \mathrm{E}+00$ \\
\hline Ionic Strength & $1.003 \mathrm{E}-02$ & $1.003 \mathrm{E}-02$ & $1.003 \mathrm{E}-02$ & $1.003 \mathrm{E}-02$ & $1.003 \mathrm{E}-02$ & $1.003 \mathrm{E}-02$ & $1.003 \mathrm{E}-02$ & $1.003 \mathrm{E}-02$ & $1.003 \mathrm{E}-02$ \\
\hline
\end{tabular}


WSRC-TR-2002-00550, Rev. 1

SRT-RPP-2002-0027.

Table B- 1. Waste Feed Evaporator 5 Stage Model Results for UF 1.22 Density Case (cont'd) ${ }^{8}$

\begin{tabular}{|c|c|c|c|c|c|c|c|c|c|}
\hline Stream & SCWash-1 & SCWash-2 & SCWash-3 & SCWash-4 & SCWash-5 & $\begin{array}{l}\text { FCWash } \\
\text { Slurry }\end{array}$ & $\begin{array}{l}\text { FCW1 } \\
\text { Liquor }\end{array}$ & $\begin{array}{l}\text { FCW1 } \\
\text { Solids }\end{array}$ & $\begin{array}{l}\text { FCW1 } \\
\text { Recycle }\end{array}$ \\
\hline Phase & Aqueous & Aqueous & Aqueous & Aqueous & Aqueous & Mixed & Aqueous & Solid & Aqueous \\
\hline Temperature, $\mathrm{C}$ & 25 & 25 & 25 & 25 & 25 & 25 & 25 & 25 & 25 \\
\hline Pressure, atm & 1 & 1 & 1 & 1 & 1 & 1 & 1 & 1 & 1 \\
\hline $\mathrm{pH}$ & 11.9511 & 11.9511 & 11.9511 & 11.9511 & 11.9511 & 13.5065 & 13.5065 & & 13.5065 \\
\hline Total mol/hr & 5.5346 & 5.5346 & 5.5346 & 5.5346 & 5.5346 & 2738.02 & 2639.05 & 98.977 & 5.52038 \\
\hline Flow Units & $\mathrm{g} / \mathrm{hr}$ & $\mathrm{g} / \mathrm{hr}$ & $\mathrm{g} / \mathrm{hr}$ & $\mathrm{g} / \mathrm{hr}$ & $\mathrm{g} / \mathrm{hr}$ & $\mathrm{g} / \mathrm{hr}$ & $\mathrm{g} / \mathrm{hr}$ & $\mathrm{g} / \mathrm{hr}$ & $\mathrm{g} / \mathrm{hr}$ \\
\hline \multicolumn{10}{|l|}{ Acetic Acid, Dimer } \\
\hline \multicolumn{10}{|l|}{ Acetic Acid } \\
\hline Aluminum Oxide & & & & & & $9.190 \mathrm{E}+01$ & $9.190 \mathrm{E}+01$ & & $1.922 \mathrm{E}-01$ \\
\hline Aldrin & & & & & & $5.222 E-04$ & $5.222 E-04$ & & $1.092 E-06$ \\
\hline Aluminum Hydrogen EDTA & & & & & & $3.101 \mathrm{E}+01$ & $3.101 \mathrm{E}+01$ & & $6.487 \mathrm{E}-02$ \\
\hline Aluminum Hydroxide & & & & & & $7.800 \mathrm{E}+02$ & & $7.800 \mathrm{E}+02$ & \\
\hline Benzene & & & & & & $2.577 E-06$ & $2.577 E-06$ & & $5.390 E-09$ \\
\hline $\begin{array}{c}\text { Bis(2-ethylhexyl)phthalate } \\
\text { (BEHP) }\end{array}$ & & & & & & $6.563 E-03$ & $6.563 E-03$ & & $1.373 E-05$ \\
\hline Benzo(a)pyrene (BaP) & & & & & & $4.180 E+00$ & $4.281 E-05$ & $4.179 E+00$ & $8.955 E-08$ \\
\hline Phenol & & & & & & $9.146 E-02$ & $9.146 E-02$ & & $1.913 E-04$ \\
\hline \multicolumn{10}{|l|}{ Calcium Chloride Oxide } \\
\hline Calcium Oxalate & & & & & & $8.946 \mathrm{E}+00$ & $8.946 \mathrm{E}+00$ & & $1.871 \mathrm{E}-02$ \\
\hline \multicolumn{10}{|l|}{ Calcium Oxalate Monohydrate } \\
\hline \multicolumn{10}{|l|}{ Calcium Carbonate } \\
\hline \multicolumn{10}{|l|}{ Calcium Dichromate (VI) } \\
\hline Calcium Fluoride & & & & & & $1.536 \mathrm{E}+03$ & & $1.536 \mathrm{E}+03$ & \\
\hline \multicolumn{10}{|l|}{ Calcium Hydroxide } \\
\hline Pentachlorophenol & & & & & & $7.898 E-03$ & $7.898 E-03$ & & $1.652 E-05$ \\
\hline Hexachlorobenzene & & & & & & $4.183 E+00$ & $1.022 E-04$ & $4.183 E+00$ & $2.138 E-07$ \\
\hline Chlorobenzene & & & & & & $5.526 E-06$ & $5.526 E-06$ & & $1.156 E-08$ \\
\hline 2-Chloronaphthalene & & & & & & $1.485 E-05$ & $1.485 E-05$ & & $3.105 E-08$ \\
\hline Carbon Dioxide & & & & & & $1.713 \mathrm{E}+03$ & $1.713 \mathrm{E}+03$ & & $3.584 \mathrm{E}+00$ \\
\hline Cesium Acetate & & & & & & $4.306 \mathrm{E}+00$ & $4.306 \mathrm{E}+00$ & & $9.007 \mathrm{E}-03$ \\
\hline \multicolumn{10}{|l|}{ Cesium Glycolate } \\
\hline Dibenz[a,h]anthracene & & & & & & $3.669 E-03$ & $3.669 E-03$ & & $7.674 E-06$ \\
\hline 1,2-dibromoethane & & & & & & $1.328 E-04$ & $1.328 E-04$ & & $2.778 E-07$ \\
\hline Diethyl phthalate & & & & & & $1.995 E-02$ & $1.995 E-02$ & & $4.173 E-05$ \\
\hline \multicolumn{10}{|l|}{ Iron (III) Oxalate } \\
\hline \multicolumn{10}{|l|}{ Iron (III) Citrate } \\
\hline Iron (III) Hydrogen EDTA & & & & & & $7.335 \mathrm{E}+00$ & $7.335 \mathrm{E}+00$ & & $1.534 \mathrm{E}-02$ \\
\hline Iron (III) Hydroxide & & & & & & $8.319 \mathrm{E}+01$ & & $8.319 \mathrm{E}+01$ & \\
\hline \multicolumn{10}{|l|}{ Formic Acid, dimer } \\
\hline \multicolumn{10}{|l|}{ Hydrofluoride, dimer } \\
\hline $2,2^{\prime}$-Iminobisacetic acid & & & & & & $2.472 \mathrm{E}+01$ & $2.472 \mathrm{E}+01$ & & $5.171 \mathrm{E}-02$ \\
\hline Water & $9.969 \mathrm{E}+01$ & $9.969 \mathrm{E}+01$ & $9.969 \mathrm{E}+01$ & $9.969 \mathrm{E}+01$ & $9.969 \mathrm{E}+01$ & $4.112 \mathrm{E}+04$ & $4.112 \mathrm{E}+04$ & & $8.602 \mathrm{E}+01$ \\
\hline \multicolumn{10}{|l|}{ Sulfuric Acid } \\
\hline Hydrogen Chloride & & & & & & $2.244 \mathrm{E}+02$ & $2.244 \mathrm{E}+02$ & & $4.695 \mathrm{E}-01$ \\
\hline Formic Acid & & & & & & $8.647 \mathrm{E}+00$ & $8.647 \mathrm{E}+00$ & & $1.809 \mathrm{E}-02$ \\
\hline Hydrofluoric Acid & & & & & & $6.675 \mathrm{E}+01$ & $6.675 \mathrm{E}+01$ & & $1.396 \mathrm{E}-01$ \\
\hline \multicolumn{10}{|l|}{ Mercury (II) Chloride } \\
\hline Mercury (elemental) & & & & & & $1.266 E-05$ & $1.266 E-05$ & & $2.647 E-08$ \\
\hline Mercury (II) Oxide & & & & & & $2.951 E+01$ & $8.674 E-01$ & $2.865 E+01$ & $1.814 E-03$ \\
\hline Nitrous (III) Acid & & & & & & $2.027 \mathrm{E}+03$ & $2.027 \mathrm{E}+03$ & & $4.239 \mathrm{E}+00$ \\
\hline Nitric Acid & & & & & & $1.392 \mathrm{E}+03$ & $1.392 \mathrm{E}+03$ & & $2.911 \mathrm{E}+00$ \\
\hline Hexachlorobutadiene & & & & & & $1.158 E-07$ & $1.158 E-07$ & & $2.423 E-10$ \\
\hline Potassium Citrate & & & & & & $6.164 \mathrm{E}+01$ & $6.164 \mathrm{E}+01$ & & $1.289 \mathrm{E}-01$ \\
\hline Potassium Acetate & & & & & & & & & \\
\hline Potassium Chloride & & & & & & $8.155 \mathrm{E}+02$ & $8.155 \mathrm{E}+02$ & & $1.706 \mathrm{E}+00$ \\
\hline
\end{tabular}


WSRC-TR-2002-00550, Rev. 1 SRT-RPP-2002-0027.

\begin{tabular}{|c|c|c|c|c|c|c|c|c|c|}
\hline Stream & SCWash-1 & SCWash-2 & SCWash-3 & SCWash-4 & SCWash-5 & $\begin{array}{l}\text { FCWash } \\
\text { Slurry }\end{array}$ & $\begin{array}{l}\text { FCW1 } \\
\text { Liquor }\end{array}$ & $\begin{array}{l}\text { FCW1 } \\
\text { Solids }\end{array}$ & $\begin{array}{l}\text { FCW1 } \\
\text { Recycle }\end{array}$ \\
\hline \multicolumn{10}{|l|}{ Potassium Glycolate } \\
\hline \multicolumn{10}{|l|}{ Potassium Nitrate (VI) } \\
\hline gamma-BHC (Lindane) & & & & & & $3.226 E-04$ & $3.226 E-04$ & & $6.748 E-07$ \\
\hline $\begin{array}{c}\text { 4-methyl-2-pentanone } \\
(\text { MIBK) }\end{array}$ & & & & & & $6.160 E-05$ & $6.160 E-05$ & & $1.288 E-07$ \\
\hline Magnesium Oxalate & & & & & & $3.468 \mathrm{E}-04$ & $3.468 \mathrm{E}-04$ & & $7.253 \mathrm{E}-07$ \\
\hline \multicolumn{10}{|l|}{ Magnesium Dichromate (VI) } \\
\hline Magnesium Hydroxide & & & & & & $2.385 \mathrm{E}+02$ & & $2.385 \mathrm{E}+02$ & \\
\hline \multicolumn{10}{|l|}{ Manganese (II) } \\
\hline \multicolumn{10}{|l|}{ Orthophosphate } \\
\hline Manganese (II) Oxalate & & & & & & $2.860 \mathrm{E}-02$ & $2.860 \mathrm{E}-02$ & & $5.983 \mathrm{E}-05$ \\
\hline \multicolumn{10}{|l|}{ Manganese (II) Hydroxide } \\
\hline Nitrogen & & & & & & $1.270 \mathrm{E}-05$ & $1.270 \mathrm{E}-05$ & & $2.657 \mathrm{E}-08$ \\
\hline Sodium Oxalate & & & & & & $8.304 \mathrm{E}+03$ & $1.184 \mathrm{E}+02$ & $8.186 \mathrm{E}+03$ & $2.477 \mathrm{E}-01$ \\
\hline Sodium Chromate (VI) & & & & & & $1.823 \mathrm{E}+02$ & $1.823 \mathrm{E}+02$ & & $3.813 \mathrm{E}-01$ \\
\hline \multicolumn{10}{|l|}{ Sodium Fluoride Sulfate } \\
\hline Sodium Acetate & & & & & & $4.007 \mathrm{E}+00$ & $4.007 \mathrm{E}+00$ & & $8.381 \mathrm{E}-03$ \\
\hline Sodium Glycolate & & & & & & $9.948 \mathrm{E}+01$ & $9.948 \mathrm{E}+01$ & & $2.081 \mathrm{E}-01$ \\
\hline Sodium Nitrite & & & & & & $1.589 \mathrm{E}+03$ & $1.589 \mathrm{E}+03$ & & $3.324 \mathrm{E}+00$ \\
\hline Sodium Hydroxide & $3.999 \mathrm{E}-02$ & $3.999 \mathrm{E}-02$ & $3.999 \mathrm{E}-02$ & $3.999 \mathrm{E}-02$ & $3.999 \mathrm{E}-02$ & $7.636 \mathrm{E}+03$ & $7.636 \mathrm{E}+03$ & & $1.597 \mathrm{E}+01$ \\
\hline Naphthalene & & & & & & $1.634 \mathrm{E}-05$ & $1.634 \mathrm{E}-05$ & & $3.417 \mathrm{E}-08$ \\
\hline Sodium Aluminosilicate Gel & & & & & & $3.690 \mathrm{E}+03$ & & $3.690 \mathrm{E}+03$ & \\
\hline \multicolumn{10}{|l|}{ Nickel Orthophosphate } \\
\hline Nickel (II) Oxalate & & & & & & $8.625 \mathrm{E}-02$ & $8.625 \mathrm{E}-02$ & & $1.804 \mathrm{E}-04$ \\
\hline \multicolumn{10}{|l|}{ Nickel (II) Hydroxide } \\
\hline Oxygen & & & & & & $7.067 \mathrm{E}-06$ & $7.067 \mathrm{E}-06$ & & $1.478 \mathrm{E}-08$ \\
\hline Phosphorus Pentoxide & & & & & & $1.395 \mathrm{E}+01$ & $1.395 \mathrm{E}+01$ & & $2.919 \mathrm{E}-02$ \\
\hline PYRENE & & & & & & $5.073 \mathrm{E}-04$ & $5.073 \mathrm{E}-04$ & & $1.061 \mathrm{E}-06$ \\
\hline Silicon Dioxide & & & & & & $1.906 \mathrm{E}+00$ & $1.906 \mathrm{E}+00$ & & $3.988 \mathrm{E}-03$ \\
\hline Sulfur Trioxide & & & & & & $1.037 \mathrm{E}+03$ & $1.037 \mathrm{E}+03$ & & $2.169 \mathrm{E}+00$ \\
\hline 1,2,4-trichlorobenzene & & & & & & $3.449 \mathrm{E}-06$ & $3.449 \mathrm{E}-06$ & & $7.215 \mathrm{E}-09$ \\
\hline 1,2,3-trichloropropane & & & & & & $2.431 \mathrm{E}-05$ & $2.431 \mathrm{E}-05$ & & 5.085E-08 \\
\hline TOLUENE & & & & & & $2.142 \mathrm{E}-06$ & $2.142 \mathrm{E}-06$ & & $4.480 \mathrm{E}-09$ \\
\hline \multicolumn{10}{|l|}{ Zinc Dichloride Pentoxide } \\
\hline Zinc Oxalate & & & & & & $2.487 \mathrm{E}+01$ & $2.487 \mathrm{E}+01$ & & $5.203 \mathrm{E}-02$ \\
\hline \multicolumn{10}{|l|}{ Zinc Hydroxide } \\
\hline Zirconium EDTA & & & & & & $3.308 \mathrm{E}-01$ & $3.308 \mathrm{E}-01$ & & 6.919E-04 \\
\hline \multicolumn{10}{|l|}{ Zirconium Oxide } \\
\hline Total g/hr & $9.973 \mathrm{E}+01$ & $9.973 \mathrm{E}+01$ & $9.973 \mathrm{E}+01$ & $9.973 \mathrm{E}+01$ & $9.973 \mathrm{E}+01$ & $7.286 \mathrm{E}+04$ & $5.831 \mathrm{E}+04$ & $1.455 \mathrm{E}+04$ & $1.220 \mathrm{E}+02$ \\
\hline Volume, L/hr & $1.000 \mathrm{E}-01$ & $1.000 \mathrm{E}-01$ & $1.000 \mathrm{E}-01$ & $1.000 \mathrm{E}-01$ & $1.000 \mathrm{E}-01$ & $5.155 \mathrm{E}+01$ & $4.781 \mathrm{E}+01$ & $3.741 \mathrm{E}+00$ & $1.000 \mathrm{E}-01$ \\
\hline Enthalpy, cal/hr & $-3.781 \mathrm{E}+05$ & $-3.781 \mathrm{E}+05$ & $-3.781 \mathrm{E}+05$ & $-3.781 \mathrm{E}+05$ & $-3.781 \mathrm{E}+05$ & $-2.354 \mathrm{E}+08$ & $-1.926 \mathrm{E}+08$ & $-4.284 \mathrm{E}+07$ & $-4.028 \mathrm{E}+05$ \\
\hline \multicolumn{10}{|l|}{ Vapor fraction } \\
\hline Solid fraction & & & & & & $3.615 \mathrm{E}-02$ & & $1.000 \mathrm{E}+00$ & \\
\hline \multicolumn{10}{|l|}{ Organic fraction } \\
\hline Osmotic Pres, atm & $4.741 \mathrm{E}-01$ & $4.741 \mathrm{E}-01$ & $4.741 \mathrm{E}-01$ & $4.741 \mathrm{E}-01$ & $4.741 \mathrm{E}-01$ & $1.736 \mathrm{E}+02$ & $1.736 \mathrm{E}+02$ & & $1.736 \mathrm{E}+02$ \\
\hline \multicolumn{10}{|l|}{ Redox Pot, volts } \\
\hline E-Con, 1/ohm-cm & $2.366 \mathrm{E}-03$ & $2.366 \mathrm{E}-03$ & $2.366 \mathrm{E}-03$ & $2.366 \mathrm{E}-03$ & $2.366 \mathrm{E}-03$ & $2.010 \mathrm{E}-01$ & $2.010 \mathrm{E}-01$ & & $2.010 \mathrm{E}-01$ \\
\hline E-Con, cm2/ohm-mol & $2.367 \mathrm{E}+02$ & $2.367 \mathrm{E}+02$ & $2.367 \mathrm{E}+02$ & $2.367 \mathrm{E}+02$ & $2.367 \mathrm{E}+02$ & $2.110 \mathrm{E}+01$ & $2.110 \mathrm{E}+01$ & & $2.110 \mathrm{E}+01$ \\
\hline Abs Visc, cP & $8.929 \mathrm{E}-01$ & $8.929 \mathrm{E}-01$ & $8.929 \mathrm{E}-01$ & $8.929 \mathrm{E}-01$ & $8.929 \mathrm{E}-01$ & $2.323 \mathrm{E}+00$ & $2.323 \mathrm{E}+00$ & & $2.323 \mathrm{E}+00$ \\
\hline Rel Visc & $1.002 \mathrm{E}+00$ & $1.002 \mathrm{E}+00$ & $1.002 \mathrm{E}+00$ & $1.002 \mathrm{E}+00$ & $1.002 \mathrm{E}+00$ & $2.608 \mathrm{E}+00$ & $2.608 \mathrm{E}+00$ & & $2.608 \mathrm{E}+00$ \\
\hline Ionic Strength & $1.003 \mathrm{E}-02$ & $1.003 \mathrm{E}-02$ & $1.003 \mathrm{E}-02$ & $1.003 \mathrm{E}-02$ & $1.003 \mathrm{E}-02$ & $5.558 \mathrm{E}+00$ & $5.558 \mathrm{E}+00$ & & $5.558 \mathrm{E}+00$ \\
\hline
\end{tabular}


WSRC-TR-2002-00550, Rev. 1

SRT-RPP-2002-0027.

Table B- 1. Waste Feed Evaporator 5 Stage Model Results for UF 1.22 Density Case (cont'd) ${ }^{8}$

\begin{tabular}{|c|c|c|c|c|c|c|c|c|c|}
\hline Stream & $\begin{array}{l}\text { FCW1 } \\
\text { Return }\end{array}$ & $\begin{array}{l}\text { FCW2 } \\
\text { Slurry }\end{array}$ & $\begin{array}{l}\text { FCW2 } \\
\text { Liquor }\end{array}$ & $\begin{array}{l}\text { FCW2 } \\
\text { Solids }\end{array}$ & $\begin{array}{l}\text { FCW2 } \\
\text { Recycle }\end{array}$ & $\begin{array}{l}\text { FCW2 } \\
\text { Return }\end{array}$ & $\begin{array}{l}\text { FCW3 } \\
\text { Slurry }\end{array}$ & $\begin{array}{l}\text { FCW3 } \\
\text { Liquor }\end{array}$ & $\begin{array}{l}\text { FCW3 } \\
\text { Solids }\end{array}$ \\
\hline Phase & Aqueous & Mixed & Aqueous & Solid & Aqueous & Aqueous & Mixed & Aqueous & Solid \\
\hline Temperature, $\mathrm{C}$ & 25 & 25 & 25 & 25 & 25 & 25 & 25 & 25 & 25 \\
\hline Pressure, atm & 1 & 1 & 1 & 1 & 1 & 1 & 1 & 1 & 1 \\
\hline $\mathrm{pH}$ & 13.5065 & 13.5047 & 13.5047 & & 13.5047 & 13.5047 & 13.5029 & 13.5029 & \\
\hline Total $\mathrm{mol} / \mathrm{hr}$ & 2633.53 & 2738.03 & 2639.06 & 98.9753 & 5.52057 & 2633.54 & 2738.05 & 2639.07 & 98.9737 \\
\hline Flow Units & $\mathrm{g} / \mathrm{hr}$ & $\mathrm{g} / \mathrm{hr}$ & $\mathrm{g} / \mathrm{hr}$ & $\mathrm{g} / \mathrm{hr}$ & $\mathrm{g} / \mathrm{hr}$ & $\mathrm{g} / \mathrm{hr}$ & $\mathrm{g} / \mathrm{hr}$ & $\mathrm{g} / \mathrm{hr}$ & $\mathrm{g} / \mathrm{hr}$ \\
\hline \multicolumn{10}{|l|}{ Acetic Acid, Dimer } \\
\hline \multicolumn{10}{|l|}{ Acetic Acid } \\
\hline Aluminum Oxide & $9.171 \mathrm{E}+01$ & $9.159 \mathrm{E}+01$ & $9.159 \mathrm{E}+01$ & & $1.916 \mathrm{E}-01$ & $9.140 \mathrm{E}+01$ & $9.128 \mathrm{E}+01$ & $9.128 \mathrm{E}+01$ & \\
\hline Aldrin & $5.211 E-04$ & $5.211 E-04$ & $5.211 E-04$ & & $1.090 E-06$ & $5.200 E-04$ & $5.200 E-04$ & $5.200 E-04$ & \\
\hline Aluminum Hydrogen EDTA & $3.095 \mathrm{E}+01$ & $3.096 \mathrm{E}+01$ & $3.096 \mathrm{E}+01$ & & $6.477 \mathrm{E}-02$ & $3.090 \mathrm{E}+01$ & $3.091 \mathrm{E}+01$ & $3.091 \mathrm{E}+01$ & \\
\hline Aluminum Hydroxide & & $7.802 \mathrm{E}+02$ & & $7.802 \mathrm{E}+02$ & & & $7.804 \mathrm{E}+02$ & & $7.804 \mathrm{E}+02$ \\
\hline Benzene & $2.571 E-06$ & $2.571 E-06$ & $2.571 E-06$ & & $5.378 E-09$ & $2.566 E-06$ & $2.566 E-06$ & $2.566 E-06$ & \\
\hline $\begin{array}{c}\text { Bis(2-ethylhexyl)phthalate } \\
\text { (BEHP) }\end{array}$ & $6.549 E-03$ & $6.549 E-03$ & $6.549 E-03$ & & $1.370 E-05$ & $6.535 E-03$ & $6.535 E-03$ & $6.535 E-03$ & \\
\hline Benzo(a)pyrene (BaP) & $4.272 E-05$ & $4.180 E+00$ & $4.289 E-05$ & $4.179 E+00$ & $8.971 E-08$ & $4.280 E-05$ & $4.180 E+00$ & $4.297 E-05$ & $4.179 E+00$ \\
\hline Phenol & $9.127 E-02$ & $9.127 E-02$ & $9.127 E-02$ & & $1.909 E-04$ & $9.108 E-02$ & $9.108 E-02$ & $9.108 E-02$ & \\
\hline \multicolumn{10}{|l|}{ Calcium Chloride Oxide } \\
\hline Calcium Oxalate & $8.927 \mathrm{E}+00$ & $8.922 \mathrm{E}+00$ & $8.922 \mathrm{E}+00$ & & $1.866 \mathrm{E}-02$ & $8.903 \mathrm{E}+00$ & $8.898 \mathrm{E}+00$ & $8.898 \mathrm{E}+00$ & \\
\hline \multicolumn{10}{|l|}{ Calcium Oxalate Monohydrate } \\
\hline \multicolumn{10}{|l|}{ Calcium Carbonate } \\
\hline \multicolumn{10}{|l|}{ Calcium Dichromate (VI) } \\
\hline Calcium Fluoride & & $1.536 \mathrm{E}+03$ & & $1.536 \mathrm{E}+03$ & & & $1.536 \mathrm{E}+03$ & & $1.536 \mathrm{E}+03$ \\
\hline \multicolumn{10}{|l|}{ Calcium Hydroxide } \\
\hline Pentachlorophenol & $7.881 E-03$ & $7.881 E-03$ & $7.881 E-03$ & & $1.649 E-05$ & $7.865 E-03$ & $7.865 E-03$ & $7.865 E-03$ & \\
\hline Hexachlorobenzene & $1.020 E-04$ & $4.183 E+00$ & $1.024 E-04$ & $4.183 E+00$ & $2.142 E-07$ & $1.022 E-04$ & $4.183 E+00$ & $1.026 E-04$ & $4.183 E+00$ \\
\hline Chlorobenzene & $5.515 E-06$ & $5.515 E-06$ & $5.515 E-06$ & & $1.154 E-08$ & $5.503 E-06$ & $5.503 E-06$ & $5.503 E-06$ & \\
\hline 2-Chloronaphthalene & $1.481 E-05$ & $1.481 E-05$ & $1.481 E-05$ & & $3.099 E-08$ & $1.478 E-05$ & $1.478 E-05$ & $1.478 E-05$ & \\
\hline Carbon Dioxide & $1.710 \mathrm{E}+03$ & $1.710 \mathrm{E}+03$ & $1.710 \mathrm{E}+03$ & & $3.576 \mathrm{E}+00$ & $1.706 \mathrm{E}+03$ & $1.706 \mathrm{E}+03$ & $1.706 \mathrm{E}+03$ & \\
\hline Cesium Acetate & $4.297 \mathrm{E}+00$ & $4.297 \mathrm{E}+00$ & $4.297 \mathrm{E}+00$ & & $8.989 \mathrm{E}-03$ & $4.288 \mathrm{E}+00$ & $4.288 \mathrm{E}+00$ & $4.288 \mathrm{E}+00$ & \\
\hline \multicolumn{10}{|l|}{ Cesium Glycolate } \\
\hline Dibenz[a,h]anthracene & $3.661 E-03$ & $3.661 E-03$ & $3.661 E-03$ & & $7.658 E-06$ & $3.653 E-03$ & $3.653 E-03$ & $3.653 E-03$ & \\
\hline 1,2-dibromoethane & $1.325 E-04$ & $1.325 E-04$ & $1.325 E-04$ & & $2.772 E-07$ & $1.323 E-04$ & $1.323 E-04$ & $1.323 E-04$ & \\
\hline Diethyl phthalate & $1.991 E-02$ & $1.991 E-02$ & $1.991 E-02$ & & $4.165 E-05$ & $1.987 E-02$ & $1.987 E-02$ & $1.987 E-02$ & \\
\hline \multicolumn{10}{|l|}{ Iron (III) Oxalate } \\
\hline \multicolumn{10}{|l|}{ Iron (III) Citrate } \\
\hline Iron (III) Hydrogen EDTA & $7.320 \mathrm{E}+00$ & $7.306 \mathrm{E}+00$ & $7.306 \mathrm{E}+00$ & & $1.528 \mathrm{E}-02$ & $7.291 \mathrm{E}+00$ & $7.278 \mathrm{E}+00$ & $7.278 \mathrm{E}+00$ & \\
\hline Iron (III) Hydroxide & & $8.319 \mathrm{E}+01$ & & $8.319 \mathrm{E}+01$ & & & $8.320 \mathrm{E}+01$ & & $8.320 \mathrm{E}+01$ \\
\hline \multicolumn{10}{|l|}{ Formic Acid, dimer } \\
\hline \multicolumn{10}{|l|}{ Hydrofluoride, dimer } \\
\hline 2,2'-Iminobisacetic acid & $2.467 \mathrm{E}+01$ & $2.467 \mathrm{E}+01$ & $2.467 \mathrm{E}+01$ & & $5.161 \mathrm{E}-02$ & $2.462 \mathrm{E}+01$ & $2.462 \mathrm{E}+01$ & $2.462 \mathrm{E}+01$ & \\
\hline Water & $4.103 \mathrm{E}+04$ & $4.113 \mathrm{E}+04$ & $4.113 \mathrm{E}+04$ & & $8.605 \mathrm{E}+01$ & $4.105 \mathrm{E}+04$ & $4.115 \mathrm{E}+04$ & $4.115 \mathrm{E}+04$ & \\
\hline \multicolumn{10}{|l|}{ Sulfuric Acid } \\
\hline Hydrogen Chloride & $2.240 \mathrm{E}+02$ & $2.240 \mathrm{E}+02$ & $2.240 \mathrm{E}+02$ & & $4.685 \mathrm{E}-01$ & $2.235 \mathrm{E}+02$ & $2.235 \mathrm{E}+02$ & $2.235 \mathrm{E}+02$ & \\
\hline Formic Acid & $8.629 \mathrm{E}+00$ & $8.629 \mathrm{E}+00$ & $8.629 \mathrm{E}+00$ & & $1.805 \mathrm{E}-02$ & $8.611 \mathrm{E}+00$ & $8.611 \mathrm{E}+00$ & $8.611 \mathrm{E}+00$ & \\
\hline Hydrofluoric Acid & $6.661 \mathrm{E}+01$ & $6.661 \mathrm{E}+01$ & $6.661 \mathrm{E}+01$ & & $1.393 \mathrm{E}-01$ & $6.647 \mathrm{E}+01$ & $6.647 \mathrm{E}+01$ & $6.647 \mathrm{E}+01$ & \\
\hline \multicolumn{10}{|l|}{ Mercury (II) Chloride } \\
\hline Mercury (elemental) & $1.263 E-05$ & $1.263 E-05$ & $1.263 E-05$ & & $2.642 E-08$ & $1.260 E-05$ & $1.260 E-05$ & $1.260 E-05$ & \\
\hline Mercury (II) Oxide & $8.655 E-01$ & $2.951 E+01$ & $8.685 E-01$ & $2.864 E+01$ & $1.817 E-03$ & $8.667 E-01$ & $2.951 E+01$ & $8.696 E-01$ & $2.864 E+01$ \\
\hline Nitrous (III) Acid & $2.022 \mathrm{E}+03$ & $2.023 \mathrm{E}+03$ & $2.023 \mathrm{E}+03$ & & $4.231 \mathrm{E}+00$ & $2.018 \mathrm{E}+03$ & $2.019 \mathrm{E}+03$ & $2.019 \mathrm{E}+03$ & \\
\hline Nitric Acid & $1.389 \mathrm{E}+03$ & $1.389 \mathrm{E}+03$ & $1.389 \mathrm{E}+03$ & & $2.905 \mathrm{E}+00$ & $1.386 \mathrm{E}+03$ & $1.386 \mathrm{E}+03$ & $1.386 \mathrm{E}+03$ & \\
\hline Hexachlorobutadiene & $1.156 E-07$ & $1.156 E-07$ & $1.156 E-07$ & & $2.418 E-10$ & $1.154 E-07$ & 1.154E-07 & $1.154 E-07$ & \\
\hline Potassium Citrate & $6.151 \mathrm{E}+01$ & $6.151 \mathrm{E}+01$ & $6.151 \mathrm{E}+01$ & & $1.287 \mathrm{E}-01$ & $6.138 \mathrm{E}+01$ & $6.138 \mathrm{E}+01$ & $6.138 \mathrm{E}+01$ & \\
\hline \multicolumn{10}{|l|}{ Potassium Acetate } \\
\hline Potassium Chloride & $8.138 \mathrm{E}+02$ & $8.138 \mathrm{E}+02$ & $8.138 \mathrm{E}+02$ & & $1.702 \mathrm{E}+00$ & $8.121 \mathrm{E}+02$ & $8.121 \mathrm{E}+02$ & $8.121 \mathrm{E}+02$ & \\
\hline
\end{tabular}


WSRC-TR-2002-00550, Rev. 1 SRT-RPP-2002-0027.

\begin{tabular}{|c|c|c|c|c|c|c|c|c|c|}
\hline Stream & $\begin{array}{l}\text { FCW1 } \\
\text { Return }\end{array}$ & $\begin{array}{l}\text { FCW2 } \\
\text { Slurry }\end{array}$ & $\begin{array}{l}\text { FCW2 } \\
\text { Liquor }\end{array}$ & $\begin{array}{l}\text { FCW2 } \\
\text { Solids }\end{array}$ & $\begin{array}{l}\text { FCW2 } \\
\text { Recycle }\end{array}$ & $\begin{array}{l}\text { FCW2 } \\
\text { Return }\end{array}$ & $\begin{array}{l}\text { FCW3 } \\
\text { Slurry }\end{array}$ & $\begin{array}{l}\text { FCW3 } \\
\text { Liquor }\end{array}$ & $\begin{array}{l}\text { FCW3 } \\
\text { Solids }\end{array}$ \\
\hline \multicolumn{10}{|l|}{ Potassium Glycolate } \\
\hline \multicolumn{10}{|l|}{ Potassium Nitrate (VI) } \\
\hline gamma-BHC (Lindane) & $3.219 E-04$ & $3.219 E-04$ & $3.219 E-04$ & & $6.734 E-07$ & $3.212 E-04$ & $3.212 E-04$ & $3.212 E-04$ & \\
\hline $\begin{array}{c}\text { 4-methyl-2-pentanone } \\
\text { (MIBK) }\end{array}$ & $6.147 E-05$ & $6.147 E-05$ & $6.147 E-05$ & & $1.286 E-07$ & $6.134 E-05$ & $6.134 E-05$ & $6.134 E-05$ & \\
\hline Magnesium Oxalate & $3.460 \mathrm{E}-04$ & $3.471 \mathrm{E}-04$ & $3.471 \mathrm{E}-04$ & & $7.261 \mathrm{E}-07$ & $3.464 \mathrm{E}-04$ & $3.475 \mathrm{E}-04$ & $3.475 \mathrm{E}-04$ & \\
\hline \multicolumn{10}{|l|}{ Magnesium Dichromate (VI) } \\
\hline Magnesium Hydroxide & & $2.385 \mathrm{E}+02$ & & $2.385 \mathrm{E}+02$ & & & $2.385 \mathrm{E}+02$ & & $2.385 \mathrm{E}+02$ \\
\hline \multicolumn{10}{|l|}{$\begin{array}{l}\text { Manganese (II) } \\
\text { Orthophosphate }\end{array}$} \\
\hline Manganese (II) Oxalate & $2.854 \mathrm{E}-02$ & $2.854 \mathrm{E}-02$ & $2.854 \mathrm{E}-02$ & & $5.971 \mathrm{E}-05$ & $2.848 \mathrm{E}-02$ & $2.848 \mathrm{E}-02$ & $2.848 \mathrm{E}-02$ & \\
\hline \multicolumn{10}{|l|}{ Manganese (II) Hydroxide } \\
\hline Nitrogen & $1.267 \mathrm{E}-05$ & $1.267 \mathrm{E}-05$ & $1.267 \mathrm{E}-05$ & & $2.651 \mathrm{E}-08$ & $1.265 \mathrm{E}-05$ & $1.265 \mathrm{E}-05$ & $1.265 \mathrm{E}-05$ & \\
\hline Sodium Oxalate & $1.182 \mathrm{E}+02$ & $8.304 \mathrm{E}+03$ & $1.187 \mathrm{E}+02$ & $8.185 \mathrm{E}+03$ & $2.484 \mathrm{E}-01$ & $1.185 \mathrm{E}+02$ & $8.304 \mathrm{E}+03$ & $1.190 \mathrm{E}+02$ & $8.185 \mathrm{E}+03$ \\
\hline Sodium Chromate (VI) & $1.819 \mathrm{E}+02$ & $1.819 \mathrm{E}+02$ & $1.819 \mathrm{E}+02$ & & $3.805 \mathrm{E}-01$ & $1.815 \mathrm{E}+02$ & $1.815 \mathrm{E}+02$ & $1.815 \mathrm{E}+02$ & \\
\hline \multicolumn{10}{|l|}{ Sodium Fluoride Sulfate } \\
\hline Sodium Acetate & $3.998 \mathrm{E}+00$ & $3.998 \mathrm{E}+00$ & $3.998 \mathrm{E}+00$ & & $8.364 \mathrm{E}-03$ & $3.990 \mathrm{E}+00$ & $3.990 \mathrm{E}+00$ & $3.990 \mathrm{E}+00$ & \\
\hline Sodium Glycolate & $9.927 \mathrm{E}+01$ & $9.927 \mathrm{E}+01$ & $9.927 \mathrm{E}+01$ & & $2.077 \mathrm{E}-01$ & $9.906 \mathrm{E}+01$ & $9.906 \mathrm{E}+01$ & $9.906 \mathrm{E}+01$ & \\
\hline Sodium Nitrite & $1.586 \mathrm{E}+03$ & $1.585 \mathrm{E}+03$ & $1.585 \mathrm{E}+03$ & & $3.316 \mathrm{E}+00$ & $1.582 \mathrm{E}+03$ & $1.582 \mathrm{E}+03$ & $1.582 \mathrm{E}+03$ & \\
\hline Sodium Hydroxide & $7.620 \mathrm{E}+03$ & $7.620 \mathrm{E}+03$ & $7.620 \mathrm{E}+03$ & & $1.594 \mathrm{E}+01$ & $7.604 \mathrm{E}+03$ & $7.605 \mathrm{E}+03$ & $7.605 \mathrm{E}+03$ & \\
\hline Naphthalene & $1.630 \mathrm{E}-05$ & $1.630 \mathrm{E}-05$ & $1.630 \mathrm{E}-05$ & & $3.410 \mathrm{E}-08$ & $1.627 \mathrm{E}-05$ & $1.627 \mathrm{E}-05$ & $1.627 \mathrm{E}-05$ & \\
\hline Sodium Aluminosilicate Gel & & $3.690 \mathrm{E}+03$ & & $3.690 \mathrm{E}+03$ & & & $3.690 \mathrm{E}+03$ & & $3.690 \mathrm{E}+03$ \\
\hline \multicolumn{10}{|l|}{ Nickel Orthophosphate } \\
\hline Nickel (II) Oxalate & $8.607 \mathrm{E}-02$ & $8.607 \mathrm{E}-02$ & $8.607 \mathrm{E}-02$ & & $1.801 \mathrm{E}-04$ & $8.589 \mathrm{E}-02$ & $8.589 \mathrm{E}-02$ & $8.589 \mathrm{E}-02$ & \\
\hline \multicolumn{10}{|l|}{ Nickel (II) Hydroxide } \\
\hline Oxygen & $7.052 \mathrm{E}-06$ & $7.052 \mathrm{E}-06$ & $7.052 \mathrm{E}-06$ & & $1.475 \mathrm{E}-08$ & $7.037 \mathrm{E}-06$ & $7.037 \mathrm{E}-06$ & $7.037 \mathrm{E}-06$ & \\
\hline Phosphorus Pentoxide & $1.393 \mathrm{E}+01$ & $1.393 \mathrm{E}+01$ & $1.393 \mathrm{E}+01$ & & $2.913 \mathrm{E}-02$ & $1.390 \mathrm{E}+01$ & $1.390 \mathrm{E}+01$ & $1.390 \mathrm{E}+01$ & \\
\hline PYRENE & $5.062 \mathrm{E}-04$ & $5.062 \mathrm{E}-04$ & $5.062 \mathrm{E}-04$ & & $1.059 \mathrm{E}-06$ & $5.052 \mathrm{E}-04$ & $5.052 \mathrm{E}-04$ & $5.052 \mathrm{E}-04$ & \\
\hline Silicon Dioxide & $1.902 \mathrm{E}+00$ & $1.903 \mathrm{E}+00$ & $1.903 \mathrm{E}+00$ & & $3.981 \mathrm{E}-03$ & $1.899 \mathrm{E}+00$ & $1.900 \mathrm{E}+00$ & $1.900 \mathrm{E}+00$ & \\
\hline Sulfur Trioxide & $1.035 \mathrm{E}+03$ & $1.035 \mathrm{E}+03$ & $1.035 \mathrm{E}+03$ & & $2.164 \mathrm{E}+00$ & $1.032 \mathrm{E}+03$ & $1.032 \mathrm{E}+03$ & $1.032 \mathrm{E}+03$ & \\
\hline 1,2,4-trichlorobenzene & $3.442 \mathrm{E}-06$ & $3.442 \mathrm{E}-06$ & $3.442 \mathrm{E}-06$ & & $7.200 \mathrm{E}-09$ & $3.435 \mathrm{E}-06$ & $3.435 \mathrm{E}-06$ & $3.435 \mathrm{E}-06$ & \\
\hline 1,2,3-trichloropropane & $2.426 \mathrm{E}-05$ & $2.426 \mathrm{E}-05$ & $2.426 \mathrm{E}-05$ & & $5.075 \mathrm{E}-08$ & $2.421 \mathrm{E}-05$ & $2.421 \mathrm{E}-05$ & $2.421 \mathrm{E}-05$ & \\
\hline TOLUENE & $2.137 \mathrm{E}-06$ & $2.137 \mathrm{E}-06$ & $2.137 \mathrm{E}-06$ & & $4.471 \mathrm{E}-09$ & $2.133 \mathrm{E}-06$ & $2.133 \mathrm{E}-06$ & $2.133 \mathrm{E}-06$ & \\
\hline \multicolumn{10}{|l|}{ Zinc Dichloride Pentoxide } \\
\hline Zinc Oxalate & $2.482 \mathrm{E}+01$ & $2.482 \mathrm{E}+01$ & $2.482 \mathrm{E}+01$ & & $5.192 \mathrm{E}-02$ & $2.477 \mathrm{E}+01$ & $2.477 \mathrm{E}+01$ & $2.477 \mathrm{E}+01$ & \\
\hline \multicolumn{10}{|l|}{ Zinc Hydroxide } \\
\hline Zirconium EDTA & $3.301 \mathrm{E}-01$ & $3.301 \mathrm{E}-01$ & $3.301 \mathrm{E}-01$ & & $6.905 \mathrm{E}-04$ & $3.294 \mathrm{E}-01$ & $3.294 \mathrm{E}-01$ & $3.294 \mathrm{E}-01$ & \\
\hline \multicolumn{10}{|l|}{ Zirconium Oxide } \\
\hline Total g/hr & $5.818 \mathrm{E}+04$ & $7.283 \mathrm{E}+04$ & $5.828 \mathrm{E}+04$ & $1.455 \mathrm{E}+04$ & $1.219 \mathrm{E}+02$ & $5.816 \mathrm{E}+04$ & $7.281 \mathrm{E}+04$ & $5.826 \mathrm{E}+04$ & $1.455 \mathrm{E}+04$ \\
\hline Volume, L/hr & $4.771 \mathrm{E}+01$ & $5.155 \mathrm{E}+01$ & $4.780 \mathrm{E}+01$ & $3.741 \mathrm{E}+00$ & $1.000 \mathrm{E}-01$ & $4.770 \mathrm{E}+01$ & $5.154 \mathrm{E}+01$ & $4.780 \mathrm{E}+01$ & $3.741 \mathrm{E}+00$ \\
\hline Enthalpy, cal/hr & $-1.922 \mathrm{E}+08$ & $-2.354 \mathrm{E}+08$ & $-1.926 \mathrm{E}+08$ & $-4.284 \mathrm{E}+07$ & $-4.028 \mathrm{E}+05$ & $-1.922 \mathrm{E}+08$ & $-2.354 \mathrm{E}+08$ & $-1.925 \mathrm{E}+08$ & $-4.284 \mathrm{E}+07$ \\
\hline \multicolumn{10}{|l|}{ Vapor fraction } \\
\hline Solid fraction & & $3.615 \mathrm{E}-02$ & & $1.000 \mathrm{E}+00$ & & & $3.615 \mathrm{E}-02$ & & $1.000 \mathrm{E}+00$ \\
\hline \multicolumn{10}{|l|}{ Organic fraction } \\
\hline Osmotic Pres, atm & $1.736 \mathrm{E}+02$ & $1.732 \mathrm{E}+02$ & $1.732 \mathrm{E}+02$ & & $1.732 \mathrm{E}+02$ & $1.732 \mathrm{E}+02$ & $1.729 \mathrm{E}+02$ & $1.729 \mathrm{E}+02$ & \\
\hline \multicolumn{10}{|l|}{ Redox Pot, volts } \\
\hline E-Con, 1/ohm-cm & $2.010 \mathrm{E}-01$ & $2.008 \mathrm{E}-01$ & $2.008 \mathrm{E}-01$ & & $2.008 \mathrm{E}-01$ & $2.008 \mathrm{E}-01$ & $2.006 \mathrm{E}-01$ & $2.006 \mathrm{E}-01$ & \\
\hline E-Con, cm2/ohm-mol & $2.110 \mathrm{E}+01$ & $2.111 \mathrm{E}+01$ & $2.111 \mathrm{E}+01$ & & $2.111 \mathrm{E}+01$ & $2.111 \mathrm{E}+01$ & $2.112 \mathrm{E}+01$ & $2.112 \mathrm{E}+01$ & \\
\hline Abs Visc, cP & $2.323 \mathrm{E}+00$ & $2.318 \mathrm{E}+00$ & $2.318 \mathrm{E}+00$ & & $2.318 \mathrm{E}+00$ & $2.318 \mathrm{E}+00$ & $2.313 \mathrm{E}+00$ & $2.313 \mathrm{E}+00$ & \\
\hline Rel Visc & $2.608 \mathrm{E}+00$ & $2.602 \mathrm{E}+00$ & $2.602 \mathrm{E}+00$ & & $2.602 \mathrm{E}+00$ & $2.602 \mathrm{E}+00$ & $2.596 \mathrm{E}+00$ & $2.596 \mathrm{E}+00$ & \\
\hline Ionic Strength & $5.558 \mathrm{E}+00$ & $5.545 \mathrm{E}+00$ & $5.545 \mathrm{E}+00$ & & $5.545 \mathrm{E}+00$ & $5.545 \mathrm{E}+00$ & $5.533 \mathrm{E}+00$ & $5.533 \mathrm{E}+00$ & \\
\hline
\end{tabular}


WSRC-TR-2002-00550, Rev. 1

SRT-RPP-2002-0027.

Table B- 1. Waste Feed Evaporator 5 Stage Model Results for UF 1.22 Density Case (cont'd) ${ }^{8}$

\begin{tabular}{|c|c|c|c|c|c|c|c|c|c|}
\hline Stream & $\begin{array}{l}\text { FCW3 } \\
\text { Recycle }\end{array}$ & $\begin{array}{l}\text { FCW3 } \\
\text { Return }\end{array}$ & $\begin{array}{l}\text { FCW4 } \\
\text { Slurry }\end{array}$ & $\begin{array}{l}\text { FCW4 } \\
\text { Liquor }\end{array}$ & $\begin{array}{l}\text { FCW4 } \\
\text { Solids }\end{array}$ & $\begin{array}{l}\text { FCW4 } \\
\text { Recycle }\end{array}$ & $\begin{array}{l}\text { FCW4 } \\
\text { Return }\end{array}$ & $\begin{array}{l}\text { FCWash5 } \\
\text { Slurry }\end{array}$ & $\begin{array}{l}\text { FCW5 } \\
\text { Liquor }\end{array}$ \\
\hline Phase & Aqueous & Aqueous & Mixed & Aqueous & Solid & Aqueous & Aqueous & Mixed & Aqueous \\
\hline Temperature, $\mathrm{C}$ & 25 & 25 & 25 & 25 & 25 & 25 & 25 & 25 & 25 \\
\hline Pressure, atm & 1 & 1 & 1 & 1 & 1 & 1 & 1 & 1 & 1 \\
\hline $\mathrm{pH}$ & 13.5029 & 13.5029 & 13.5012 & 13.5012 & & 13.5012 & 13.5012 & 13.4994 & 13.4994 \\
\hline Total mol/hr & 5.52076 & 2633.55 & 2738.06 & 2639.09 & 98.972 & 5.52096 & 2633.56 & 2738.07 & 2639.1 \\
\hline Flow Units & $\mathrm{g} / \mathrm{hr}$ & $\mathrm{g} / \mathrm{hr}$ & $\mathrm{g} / \mathrm{hr}$ & $\mathrm{g} / \mathrm{hr}$ & $\mathrm{g} / \mathrm{hr}$ & $\mathrm{g} / \mathrm{hr}$ & $\mathrm{g} / \mathrm{hr}$ & $\mathrm{g} / \mathrm{hr}$ & $\mathrm{g} / \mathrm{hr}$ \\
\hline \multicolumn{10}{|l|}{ Acetic Acid, Dimer } \\
\hline \multicolumn{10}{|l|}{ Acetic Acid } \\
\hline Aluminum Oxide & $1.909 \mathrm{E}-01$ & $9.109 \mathrm{E}+01$ & $9.097 \mathrm{E}+01$ & $9.097 \mathrm{E}+01$ & & $1.903 \mathrm{E}-01$ & $9.078 \mathrm{E}+01$ & $9.066 \mathrm{E}+01$ & $9.066 \mathrm{E}+01$ \\
\hline Aldrin & $1.088 E-06$ & $5.190 E-04$ & $5.189 E-04$ & $5.190 E-04$ & & $1.086 E-06$ & $5.179 E-04$ & $5.179 E-04$ & $5.179 E-04$ \\
\hline Aluminum Hydrogen EDTA & $6.466 \mathrm{E}-02$ & $3.084 \mathrm{E}+01$ & $3.086 \mathrm{E}+01$ & $3.086 \mathrm{E}+01$ & & $6.455 \mathrm{E}-02$ & $3.079 \mathrm{E}+01$ & $3.080 \mathrm{E}+01$ & $3.080 \mathrm{E}+01$ \\
\hline Aluminum Hydroxide & & & $7.806 \mathrm{E}+02$ & & $7.806 \mathrm{E}+02$ & & & $7.808 \mathrm{E}+02$ & \\
\hline Benzene & $5.367 E-09$ & $2.560 E-06$ & $2.560 E-06$ & $2.560 E-06$ & & $5.356 E-09$ & $2.555 E-06$ & $2.555 E-06$ & $2.555 E-06$ \\
\hline $\begin{array}{c}\text { Bis(2-ethylhexyl)phthalate } \\
\text { (BEHP) }\end{array}$ & $1.367 E-05$ & $6.521 E-03$ & $6.521 E-03$ & $6.521 E-03$ & & $1.364 E-05$ & $6.508 E-03$ & $6.508 E-03$ & $6.508 E-03$ \\
\hline Benzo(a)pyrene (BaP) & $8.988 E-08$ & $4.288 E-05$ & $4.180 E+00$ & $4.304 E-05$ & $4.180 E+00$ & $9.005 E-08$ & $4.295 E-05$ & $4.180 E+00$ & $4.312 E-05$ \\
\hline Phenol & $1.905 E-04$ & $9.089 E-02$ & $9.089 E-02$ & $9.089 E-02$ & & $1.901 E-04$ & $9.070 E-02$ & $9.070 E-02$ & $9.070 E-02$ \\
\hline \multicolumn{10}{|l|}{ Calcium Chloride Oxide } \\
\hline Calcium Oxalate & $1.861 \mathrm{E}-02$ & $8.880 \mathrm{E}+00$ & $8.875 \mathrm{E}+00$ & $8.875 \mathrm{E}+00$ & & $1.857 \mathrm{E}-02$ & $8.856 \mathrm{E}+00$ & $8.851 \mathrm{E}+00$ & $8.851 \mathrm{E}+00$ \\
\hline \multicolumn{10}{|l|}{ Calcium Oxalate Monohydrate } \\
\hline \multicolumn{10}{|l|}{ Calcium Carbonate } \\
\hline \multicolumn{10}{|l|}{ Calcium Dichromate (VI) } \\
\hline Calcium Fluoride & & & $1.536 \mathrm{E}+03$ & & $1.536 \mathrm{E}+03$ & & & $1.536 \mathrm{E}+03$ & \\
\hline \multicolumn{10}{|l|}{ Calcium Hydroxide } \\
\hline Pentachlorophenol & $1.645 E-05$ & $7.848 E-03$ & $7.848 E-03$ & $7.848 E-03$ & & $1.642 E-05$ & $7.832 E-03$ & $7.832 E-03$ & $7.832 E-03$ \\
\hline Hexachlorobenzene & $2.146 E-07$ & $1.023 E-04$ & $4.183 E+00$ & $1.028 E-04$ & $4.183 E+00$ & $2.150 E-07$ & $1.025 E-04$ & $4.183 E+00$ & $1.029 E-04$ \\
\hline Chlorobenzene & $1.151 E-08$ & $5.492 E-06$ & $5.492 E-06$ & $5.492 E-06$ & & $1.149 E-08$ & $5.480 E-06$ & $5.480 E-06$ & $5.480 E-06$ \\
\hline 2-Chloronaphthalene & $3.093 E-08$ & $1.475 E-05$ & $1.475 E-05$ & $1.475 E-05$ & & $3.086 E-08$ & $1.472 E-05$ & $1.472 E-05$ & $1.472 E-05$ \\
\hline Carbon Dioxide & $3.569 \mathrm{E}+00$ & $1.703 \mathrm{E}+03$ & $1.703 \mathrm{E}+03$ & $1.703 \mathrm{E}+03$ & & $3.562 \mathrm{E}+00$ & $1.699 \mathrm{E}+03$ & $1.699 \mathrm{E}+03$ & $1.699 \mathrm{E}+03$ \\
\hline Cesium Acetate & $8.970 \mathrm{E}-03$ & $4.279 \mathrm{E}+00$ & $4.279 \mathrm{E}+00$ & $4.279 \mathrm{E}+00$ & & $8.952 \mathrm{E}-03$ & $4.270 \mathrm{E}+00$ & $4.270 \mathrm{E}+00$ & $4.270 \mathrm{E}+00$ \\
\hline \multicolumn{10}{|l|}{ Cesium Glycolate } \\
\hline Dibenz[a,h]anthracene & $7.642 E-06$ & $3.646 E-03$ & $3.646 E-03$ & $3.646 E-03$ & & $7.627 E-06$ & $3.638 E-03$ & $3.638 E-03$ & $3.638 E-03$ \\
\hline 1,2-dibromoethane & $2.767 E-07$ & $1.320 E-04$ & $1.320 E-04$ & $1.320 E-04$ & & $2.761 E-07$ & $1.317 E-04$ & $1.317 E-04$ & $1.317 E-04$ \\
\hline Diethyl phthalate & $4.156 E-05$ & $1.983 E-02$ & $1.983 E-02$ & $1.983 E-02$ & & $4.148 E-05$ & $1.978 E-02$ & $1.978 E-02$ & $1.978 E-02$ \\
\hline \multicolumn{10}{|l|}{ Iron (III) Oxalate } \\
\hline \multicolumn{10}{|l|}{ Iron (III) Citrate } \\
\hline Iron (III) Hydrogen EDTA & $1.522 \mathrm{E}-02$ & $7.262 \mathrm{E}+00$ & $7.249 \mathrm{E}+00$ & $7.249 \mathrm{E}+00$ & & $1.516 \mathrm{E}-02$ & $7.234 \mathrm{E}+00$ & $7.221 \mathrm{E}+00$ & $7.221 \mathrm{E}+00$ \\
\hline Iron (III) Hydroxide & & & $8.320 \mathrm{E}+01$ & & $8.320 \mathrm{E}+01$ & & & $8.320 \mathrm{E}+01$ & \\
\hline \multicolumn{10}{|l|}{ Formic Acid, dimer } \\
\hline \multicolumn{10}{|l|}{ Hydrofluoride, dimer } \\
\hline $2,2^{\prime}$-Iminobisacetic acid & $5.150 \mathrm{E}-02$ & $2.457 \mathrm{E}+01$ & $2.457 \mathrm{E}+01$ & $2.457 \mathrm{E}+01$ & & $5.140 \mathrm{E}-02$ & $2.452 \mathrm{E}+01$ & $2.452 \mathrm{E}+01$ & $2.452 \mathrm{E}+01$ \\
\hline Water & $8.608 \mathrm{E}+01$ & $4.106 \mathrm{E}+04$ & $4.116 \mathrm{E}+04$ & $4.116 \mathrm{E}+04$ & & $8.611 \mathrm{E}+01$ & $4.108 \mathrm{E}+04$ & $4.117 \mathrm{E}+04$ & $4.117 \mathrm{E}+04$ \\
\hline \multicolumn{10}{|l|}{ Sulfuric Acid } \\
\hline Hydrogen Chloride & $4.676 \mathrm{E}-01$ & $2.230 \mathrm{E}+02$ & $2.230 \mathrm{E}+02$ & $2.230 \mathrm{E}+02$ & & $4.666 \mathrm{E}-01$ & $2.226 \mathrm{E}+02$ & $2.226 \mathrm{E}+02$ & $2.226 \mathrm{E}+02$ \\
\hline Formic Acid & $1.801 \mathrm{E}-02$ & $8.593 \mathrm{E}+00$ & $8.593 \mathrm{E}+00$ & $8.593 \mathrm{E}+00$ & & $1.798 \mathrm{E}-02$ & $8.575 \mathrm{E}+00$ & $8.575 \mathrm{E}+00$ & $8.575 \mathrm{E}+00$ \\
\hline Hydrofluoric Acid & $1.390 \mathrm{E}-01$ & $6.633 \mathrm{E}+01$ & $6.633 \mathrm{E}+01$ & $6.633 \mathrm{E}+01$ & & $1.388 \mathrm{E}-01$ & $6.619 \mathrm{E}+01$ & $6.619 \mathrm{E}+01$ & $6.619 \mathrm{E}+01$ \\
\hline \multicolumn{10}{|l|}{ Mercury (II) Chloride } \\
\hline Mercury (elemental) & $2.636 E-08$ & $1.258 E-05$ & $1.258 E-05$ & $1.258 E-05$ & & $2.631 E-08$ & $1.255 E-05$ & $1.255 E-05$ & $1.255 E-05$ \\
\hline Mercury (II) Oxide & $1.819 E-03$ & $8.678 E-01$ & $2.951 E+01$ & $8.708 E-01$ & $2.864 E+01$ & $1.822 E-03$ & $8.689 E-01$ & $2.951 E+01$ & $8.719 E-01$ \\
\hline Nitrous (III) Acid & $4.223 \mathrm{E}+00$ & $2.014 \mathrm{E}+03$ & $2.015 \mathrm{E}+03$ & $2.015 \mathrm{E}+03$ & & $4.215 \mathrm{E}+00$ & $2.010 \mathrm{E}+03$ & $2.011 \mathrm{E}+03$ & $2.011 \mathrm{E}+03$ \\
\hline Nitric Acid & $2.899 \mathrm{E}+00$ & $1.383 \mathrm{E}+03$ & $1.383 \mathrm{E}+03$ & $1.383 \mathrm{E}+03$ & & $2.894 \mathrm{E}+00$ & $1.380 \mathrm{E}+03$ & $1.380 \mathrm{E}+03$ & $1.380 \mathrm{E}+03$ \\
\hline Hexachlorobutadiene & $2.413 E-10$ & $1.151 E-07$ & $1.151 E-07$ & $1.151 E-07$ & & $2.408 E-10$ & $1.149 E-07$ & $1.149 E-07$ & $1.149 E-07$ \\
\hline Potassium Citrate & $1.284 \mathrm{E}-01$ & $6.125 \mathrm{E}+01$ & $6.125 \mathrm{E}+01$ & $6.125 \mathrm{E}+01$ & & $1.281 \mathrm{E}-01$ & $6.112 \mathrm{E}+01$ & $6.113 \mathrm{E}+01$ & $6.113 \mathrm{E}+01$ \\
\hline \multicolumn{10}{|l|}{ Potassium Acetate } \\
\hline Potassium Chloride & $1.699 \mathrm{E}+00$ & $8.104 \mathrm{E}+02$ & $8.104 \mathrm{E}+02$ & $8.104 \mathrm{E}+02$ & & $1.695 \mathrm{E}+00$ & $8.087 \mathrm{E}+02$ & $8.087 \mathrm{E}+02$ & $8.087 \mathrm{E}+02$ \\
\hline
\end{tabular}


WSRC-TR-2002-00550, Rev. 1

SRT-RPP-2002-0027.

\begin{tabular}{|c|c|c|c|c|c|c|c|c|c|}
\hline Stream & $\begin{array}{l}\text { FCW3 } \\
\text { Recycle }\end{array}$ & $\begin{array}{l}\text { FCW3 } \\
\text { Return }\end{array}$ & $\begin{array}{l}\text { FCW4 } \\
\text { Slurry }\end{array}$ & $\begin{array}{l}\text { FCW4 } \\
\text { Liquor }\end{array}$ & $\begin{array}{l}\text { FCW4 } \\
\text { Solids }\end{array}$ & $\begin{array}{l}\text { FCW4 } \\
\text { Recycle }\end{array}$ & $\begin{array}{l}\text { FCW4 } \\
\text { Return }\end{array}$ & $\begin{array}{l}\text { FCWash5 } \\
\text { Slurry }\end{array}$ & $\begin{array}{l}\text { FCW5 } \\
\text { Liquor }\end{array}$ \\
\hline \multicolumn{10}{|l|}{ Potassium Glycolate } \\
\hline \multicolumn{10}{|l|}{ Potassium Nitrate (VI) } \\
\hline gamma-BHC (Lindane) & $6.720 E-07$ & $3.206 E-04$ & $3.206 E-04$ & $3.206 E-04$ & & $6.706 E-07$ & $3.199 E-04$ & $3.199 E-04$ & $3.199 E-04$ \\
\hline $\begin{array}{c}\text { 4-methyl-2-pentanone } \\
\text { (MIBK) }\end{array}$ & $1.283 E-07$ & $6.121 E-05$ & $6.121 E-05$ & $6.121 E-05$ & & $1.281 E-07$ & $6.108 E-05$ & $6.108 E-05$ & $6.108 E-05$ \\
\hline Magnesium Oxalate & $7.269 \mathrm{E}-07$ & $3.468 \mathrm{E}-04$ & $3.479 \mathrm{E}-04$ & $3.479 \mathrm{E}-04$ & & $7.277 \mathrm{E}-07$ & $3.471 \mathrm{E}-04$ & $3.482 \mathrm{E}-04$ & $3.482 \mathrm{E}-04$ \\
\hline \multicolumn{10}{|l|}{ Magnesium Dichromate (VI) } \\
\hline Magnesium Hydroxide & & & $2.385 \mathrm{E}+02$ & & $2.385 \mathrm{E}+02$ & & & $2.385 \mathrm{E}+02$ & \\
\hline \multicolumn{10}{|l|}{$\begin{array}{l}\text { Manganese (II) } \\
\text { Orthophosphate }\end{array}$} \\
\hline Manganese (II) Oxalate & 5.959E-05 & $2.842 \mathrm{E}-02$ & $2.842 \mathrm{E}-02$ & $2.842 \mathrm{E}-02$ & & $5.946 \mathrm{E}-05$ & $2.837 \mathrm{E}-02$ & $2.837 \mathrm{E}-02$ & $2.837 \mathrm{E}-02$ \\
\hline \multicolumn{10}{|l|}{ Manganese (II) Hydroxide } \\
\hline Nitrogen & $2.646 \mathrm{E}-08$ & $1.262 \mathrm{E}-05$ & $1.262 \mathrm{E}-05$ & $1.262 \mathrm{E}-05$ & & $2.640 \mathrm{E}-08$ & $1.260 \mathrm{E}-05$ & $1.260 \mathrm{E}-05$ & $1.260 \mathrm{E}-05$ \\
\hline Sodium Oxalate & $2.490 \mathrm{E}-01$ & $1.188 \mathrm{E}+02$ & $8.304 \mathrm{E}+03$ & $1.193 \mathrm{E}+02$ & $8.184 \mathrm{E}+03$ & $2.496 \mathrm{E}-01$ & $1.191 \mathrm{E}+02$ & $8.303 \mathrm{E}+03$ & $1.196 \mathrm{E}+02$ \\
\hline Sodium Chromate (VI) & $3.797 \mathrm{E}-01$ & $1.811 \mathrm{E}+02$ & $1.811 \mathrm{E}+02$ & $1.811 \mathrm{E}+02$ & & $3.790 \mathrm{E}-01$ & $1.808 \mathrm{E}+02$ & $1.808 \mathrm{E}+02$ & $1.808 \mathrm{E}+02$ \\
\hline \multicolumn{10}{|l|}{ Sodium Fluoride Sulfate } \\
\hline Sodium Acetate & $8.347 \mathrm{E}-03$ & $3.982 \mathrm{E}+00$ & $3.982 \mathrm{E}+00$ & $3.982 \mathrm{E}+00$ & & $8.329 \mathrm{E}-03$ & $3.973 \mathrm{E}+00$ & $3.973 \mathrm{E}+00$ & $3.973 \mathrm{E}+00$ \\
\hline Sodium Glycolate & $2.072 \mathrm{E}-01$ & $9.886 \mathrm{E}+01$ & $9.886 \mathrm{E}+01$ & $9.886 \mathrm{E}+01$ & & $2.068 \mathrm{E}-01$ & $9.865 \mathrm{E}+01$ & $9.865 \mathrm{E}+01$ & $9.865 \mathrm{E}+01$ \\
\hline Sodium Nitrite & $3.308 \mathrm{E}+00$ & $1.578 \mathrm{E}+03$ & $1.578 \mathrm{E}+03$ & $1.578 \mathrm{E}+03$ & & $3.301 \mathrm{E}+00$ & $1.574 \mathrm{E}+03$ & $1.574 \mathrm{E}+03$ & $1.574 \mathrm{E}+03$ \\
\hline Sodium Hydroxide & $1.591 \mathrm{E}+01$ & $7.589 \mathrm{E}+03$ & $7.589 \mathrm{E}+03$ & $7.589 \mathrm{E}+03$ & & $1.588 \mathrm{E}+01$ & $7.573 \mathrm{E}+03$ & $7.573 \mathrm{E}+03$ & $7.573 \mathrm{E}+03$ \\
\hline Naphthalene & $3.403 \mathrm{E}-08$ & $1.623 \mathrm{E}-05$ & $1.623 \mathrm{E}-05$ & $1.623 \mathrm{E}-05$ & & $3.396 \mathrm{E}-08$ & $1.620 \mathrm{E}-05$ & $1.620 \mathrm{E}-05$ & $1.620 \mathrm{E}-05$ \\
\hline Sodium Aluminosilicate Gel & & & $3.690 \mathrm{E}+03$ & & $3.690 \mathrm{E}+03$ & & & $3.690 \mathrm{E}+03$ & \\
\hline \multicolumn{10}{|l|}{ Nickel Orthophosphate } \\
\hline Nickel (II) Oxalate & $1.797 \mathrm{E}-04$ & $8.571 \mathrm{E}-02$ & $8.571 \mathrm{E}-02$ & $8.571 \mathrm{E}-02$ & & $1.793 \mathrm{E}-04$ & $8.553 \mathrm{E}-02$ & $8.553 \mathrm{E}-02$ & $8.553 \mathrm{E}-02$ \\
\hline \multicolumn{10}{|l|}{ Nickel (II) Hydroxide } \\
\hline Oxygen & $1.472 \mathrm{E}-08$ & $7.022 \mathrm{E}-06$ & $7.022 \mathrm{E}-06$ & $7.022 \mathrm{E}-06$ & & $1.469 \mathrm{E}-08$ & $7.008 \mathrm{E}-06$ & $7.008 \mathrm{E}-06$ & $7.008 \mathrm{E}-06$ \\
\hline Phosphorus Pentoxide & $2.907 \mathrm{E}-02$ & $1.387 \mathrm{E}+01$ & $1.387 \mathrm{E}+01$ & $1.387 \mathrm{E}+01$ & & $2.901 \mathrm{E}-02$ & $1.384 \mathrm{E}+01$ & $1.384 \mathrm{E}+01$ & $1.384 \mathrm{E}+01$ \\
\hline PYRENE & $1.057 \mathrm{E}-06$ & $5.041 \mathrm{E}-04$ & $5.041 \mathrm{E}-04$ & $5.041 \mathrm{E}-04$ & & $1.055 \mathrm{E}-06$ & $5.030 \mathrm{E}-04$ & $5.030 \mathrm{E}-04$ & $5.030 \mathrm{E}-04$ \\
\hline Silicon Dioxide & $3.974 \mathrm{E}-03$ & $1.896 \mathrm{E}+00$ & $1.896 \mathrm{E}+00$ & $1.896 \mathrm{E}+00$ & & $3.967 \mathrm{E}-03$ & $1.892 \mathrm{E}+00$ & $1.893 \mathrm{E}+00$ & $1.893 \mathrm{E}+00$ \\
\hline Sulfur Trioxide & $2.160 \mathrm{E}+00$ & $1.030 \mathrm{E}+03$ & $1.030 \mathrm{E}+03$ & $1.030 \mathrm{E}+03$ & & $2.155 \mathrm{E}+00$ & $1.028 \mathrm{E}+03$ & $1.028 \mathrm{E}+03$ & $1.028 \mathrm{E}+03$ \\
\hline 1,2,4-trichlorobenzene & $7.185 \mathrm{E}-09$ & $3.427 \mathrm{E}-06$ & $3.427 \mathrm{E}-06$ & $3.427 \mathrm{E}-06$ & & $7.170 \mathrm{E}-09$ & $3.420 \mathrm{E}-06$ & $3.420 \mathrm{E}-06$ & $3.420 \mathrm{E}-06$ \\
\hline 1,2,3-trichloropropane & $5.064 \mathrm{E}-08$ & $2.416 \mathrm{E}-05$ & $2.416 \mathrm{E}-05$ & $2.416 \mathrm{E}-05$ & & $5.054 \mathrm{E}-08$ & $2.411 \mathrm{E}-05$ & $2.411 \mathrm{E}-05$ & $2.411 \mathrm{E}-05$ \\
\hline TOLUENE & $4.462 \mathrm{E}-09$ & $2.128 \mathrm{E}-06$ & $2.128 \mathrm{E}-06$ & $2.128 \mathrm{E}-06$ & & $4.453 \mathrm{E}-09$ & $2.124 \mathrm{E}-06$ & $2.124 \mathrm{E}-06$ & $2.124 \mathrm{E}-06$ \\
\hline \multicolumn{10}{|l|}{ Zinc Dichloride Pentoxide } \\
\hline Zinc Oxalate & $5.182 \mathrm{E}-02$ & $2.472 \mathrm{E}+01$ & $2.472 \mathrm{E}+01$ & $2.472 \mathrm{E}+01$ & & $5.171 \mathrm{E}-02$ & $2.467 \mathrm{E}+01$ & $2.467 \mathrm{E}+01$ & $2.467 \mathrm{E}+01$ \\
\hline \multicolumn{10}{|l|}{ Zinc Hydroxide } \\
\hline Zirconium EDTA & $6.890 \mathrm{E}-04$ & $3.287 \mathrm{E}-01$ & $3.287 \mathrm{E}-01$ & $3.287 \mathrm{E}-01$ & & $6.876 \mathrm{E}-04$ & $3.280 \mathrm{E}-01$ & $3.280 \mathrm{E}-01$ & $3.280 \mathrm{E}-01$ \\
\hline \multicolumn{10}{|l|}{ Zirconium Oxide } \\
\hline Total g/hr & $1.219 \mathrm{E}+02$ & $5.814 \mathrm{E}+04$ & $7.279 \mathrm{E}+04$ & $5.824 \mathrm{E}+04$ & $1.455 \mathrm{E}+04$ & $1.218 \mathrm{E}+02$ & $5.812 \mathrm{E}+04$ & $7.277 \mathrm{E}+04$ & $5.822 \mathrm{E}+04$ \\
\hline Volume, L/hr & $1.000 \mathrm{E}-01$ & $4.770 \mathrm{E}+01$ & $5.154 \mathrm{E}+01$ & $4.780 \mathrm{E}+01$ & $3.741 \mathrm{E}+00$ & $1.000 \mathrm{E}-01$ & $4.770 \mathrm{E}+01$ & $5.154 \mathrm{E}+01$ & $4.780 \mathrm{E}+01$ \\
\hline Enthalpy, cal/hr & $-4.028 \mathrm{E}+05$ & $-1.921 \mathrm{E}+08$ & $-2.353 \mathrm{E}+08$ & $-1.925 \mathrm{E}+08$ & $-4.284 \mathrm{E}+07$ & $-4.027 \mathrm{E}+05$ & $-1.921 \mathrm{E}+08$ & $-2.353 \mathrm{E}+08$ & $-1.925 \mathrm{E}+08$ \\
\hline \multicolumn{10}{|l|}{ Vapor fraction } \\
\hline Solid fraction & & & $3.615 \mathrm{E}-02$ & & $1.000 \mathrm{E}+00$ & & & $3.615 \mathrm{E}-02$ & \\
\hline \multicolumn{10}{|l|}{ Organic fraction } \\
\hline Osmotic Pres, atm & $1.729 \mathrm{E}+02$ & $1.729 \mathrm{E}+02$ & $1.725 \mathrm{E}+02$ & $1.725 \mathrm{E}+02$ & & $1.725 \mathrm{E}+02$ & $1.725 \mathrm{E}+02$ & $1.722 \mathrm{E}+02$ & $1.722 \mathrm{E}+02$ \\
\hline \multicolumn{10}{|l|}{ Redox Pot, volts } \\
\hline E-Con, 1/ohm-cm & $2.006 \mathrm{E}-01$ & $2.006 \mathrm{E}-01$ & $2.003 \mathrm{E}-01$ & $2.003 \mathrm{E}-01$ & & $2.003 \mathrm{E}-01$ & $2.003 \mathrm{E}-01$ & $2.001 \mathrm{E}-01$ & 2.001E-01 \\
\hline E-Con, cm2/ohm-mol & $2.112 \mathrm{E}+01$ & $2.112 \mathrm{E}+01$ & $2.113 \mathrm{E}+01$ & $2.113 \mathrm{E}+01$ & & $2.113 \mathrm{E}+01$ & $2.113 \mathrm{E}+01$ & $2.114 \mathrm{E}+01$ & $2.114 \mathrm{E}+01$ \\
\hline Abs Visc, cP & $2.313 \mathrm{E}+00$ & $2.313 \mathrm{E}+00$ & $2.308 \mathrm{E}+00$ & $2.308 \mathrm{E}+00$ & & $2.308 \mathrm{E}+00$ & $2.308 \mathrm{E}+00$ & $2.303 \mathrm{E}+00$ & $2.303 \mathrm{E}+00$ \\
\hline Rel Visc & $2.596 \mathrm{E}+00$ & $2.596 \mathrm{E}+00$ & $2.591 \mathrm{E}+00$ & $2.591 \mathrm{E}+00$ & & $2.591 \mathrm{E}+00$ & $2.591 \mathrm{E}+00$ & $2.585 \mathrm{E}+00$ & $2.585 \mathrm{E}+00$ \\
\hline Ionic Strength & $5.533 \mathrm{E}+00$ & $5.533 \mathrm{E}+00$ & $5.520 \mathrm{E}+00$ & $5.520 \mathrm{E}+00$ & & $5.520 \mathrm{E}+00$ & $5.520 \mathrm{E}+00$ & $5.508 \mathrm{E}+00$ & $5.508 \mathrm{E}+00$ \\
\hline
\end{tabular}


WSRC-TR-2002-00550, Rev. 1

SRT-RPP-2002-0027.

Table B- 1. Waste Feed Evaporator 5 Stage Model Results for UF 1.22 Density Case (cont'd) ${ }^{8}$

\begin{tabular}{|c|c|c|c|c|c|c|c|c|c|}
\hline Stream & $\begin{array}{l}\text { FCW5 } \\
\text { Solids }\end{array}$ & $\begin{array}{l}\text { FCW5 } \\
\text { Recycle }\end{array}$ & $\begin{array}{l}\text { FCW5 } \\
\text { Return }\end{array}$ & Leach-in & Total Leach & $\begin{array}{l}\text { FCWash- } \\
\text { Leach }\end{array}$ & Steam-in & Total Steam & UF2 Filtrate \\
\hline Phase & Solid & Aqueous & Aqueous & Aqueous & Aqueous & Mixed & Aqueous & Aqueous & Aqueous \\
\hline Temperature, $\mathrm{C}$ & 25 & 25 & 25 & 25 & 25 & 25 & 25 & 25 & 25 \\
\hline Pressure, atm & 1 & 1 & 1 & 1 & 1 & 1 & 1 & 1 & 1 \\
\hline $\mathrm{pH}$ & & 13.4994 & 13.4994 & 17.8006 & 17.8006 & 14.9827 & 6.9969 & 6.9969 & 14.8733 \\
\hline Total $\mathrm{mol} / \mathrm{hr}$ & 98.9703 & 5.52114 & 2633.58 & 62.1856 & 608.973 & 3342.56 & 55.3332 & 395.386 & 1041.54 \\
\hline Flow Units & $\mathrm{g} / \mathrm{hr}$ & $\mathrm{g} / \mathrm{hr}$ & $\mathrm{g} / \mathrm{hr}$ & $\mathrm{g} / \mathrm{hr}$ & $\mathrm{g} / \mathrm{hr}$ & $\mathrm{g} / \mathrm{hr}$ & $\mathrm{g} / \mathrm{hr}$ & $\mathrm{g} / \mathrm{hr}$ & $\mathrm{g} / \mathrm{hr}$ \\
\hline Acetic Acid, Dimer & & & & & & & & & \\
\hline Acetic Acid & & & & & & $4.236 \mathrm{E}+00$ & & & $1.208 \mathrm{E}+00$ \\
\hline Aluminum Oxide & & $1.897 \mathrm{E}-01$ & $9.047 \mathrm{E}+01$ & & & $6.958 \mathrm{E}+02$ & & & $1.945 \mathrm{E}+02$ \\
\hline Aldrin & & $1.083 E-06$ & $5.168 E-04$ & & & $5.168 E-04$ & & & $1.474 E-04$ \\
\hline Aluminum Hydrogen EDTA & & $6.444 \mathrm{E}-02$ & $3.074 \mathrm{E}+01$ & & & & & & \\
\hline Aluminum Hydroxide & $7.808 \mathrm{E}+02$ & & & & & & & & \\
\hline Benzene & & $5.345 E-09$ & $2.550 E-06$ & & & $2.550 E-06$ & & & $7.273 E-07$ \\
\hline $\begin{array}{c}\text { Bis(2-ethylhexyl)phthalate } \\
\text { (BEHP) }\end{array}$ & & $1.361 E-05$ & $6.494 E-03$ & & & $6.494 E-03$ & & & $1.852 E-03$ \\
\hline Benzo(a)pyrene (BaP) & $4.179 E+00$ & $9.021 E-08$ & $4.303 E-05$ & & & $4.180 E+00$ & & & $8.551 E-06$ \\
\hline Phenol & & $1.898 E-04$ & $9.051 E-02$ & & & $9.051 E-02$ & & & $2.582 E-02$ \\
\hline Calcium Chloride Oxide & & & & & & & & & \\
\hline Calcium Oxalate & & $1.852 \mathrm{E}-02$ & $8.832 \mathrm{E}+00$ & & & & & & \\
\hline Calcium Oxalate Monohydrate & & & & & & & & & \\
\hline Calcium Carbonate & & & & & & & & & \\
\hline Calcium Dichromate (VI) & & & & & & $2.837 \mathrm{E}+01$ & & & $8.335 \mathrm{E}+00$ \\
\hline Calcium Fluoride & $1.536 \mathrm{E}+03$ & & & & & $1.214 \mathrm{E}+03$ & & & \\
\hline Calcium Hydroxide & & & & & & $3.032 \mathrm{E}+02$ & & & \\
\hline Pentachlorophenol & & $1.638 E-05$ & $7.815 E-03$ & & & $7.815 E-03$ & & & $2.229 E-03$ \\
\hline Hexachlorobenzene & $4.183 E+00$ & $2.153 E-07$ & $1.027 E-04$ & & & $4.183 E+00$ & & & $2.041 E-05$ \\
\hline Chlorobenzene & & $1.146 E-08$ & $5.469 E-06$ & & & $5.469 E-06$ & & & $1.560 E-06$ \\
\hline 2-Chloronaphthalene & & $3.080 E-08$ & $1.469 E-05$ & & & $1.469 E-05$ & & & $4.191 E-06$ \\
\hline Carbon Dioxide & & $3.554 \mathrm{E}+00$ & $1.695 \mathrm{E}+03$ & & & $1.695 \mathrm{E}+03$ & & & $4.836 \mathrm{E}+02$ \\
\hline Cesium Acetate & & $8.933 \mathrm{E}-03$ & $4.261 \mathrm{E}+00$ & & & & & & \\
\hline Cesium Glycolate & & & & & & $4.616 \mathrm{E}+00$ & & & $1.317 \mathrm{E}+00$ \\
\hline Dibenz[a,h]anthracene & & $7.611 E-06$ & $3.630 E-03$ & & & $3.630 E-03$ & & & $1.036 E-03$ \\
\hline 1,2-dibromoethane & & $2.755 E-07$ & $1.314 E-04$ & & & $1.314 E-04$ & & & $3.749 E-05$ \\
\hline Diethyl phthalate & & $4.139 E-05$ & $1.974 E-02$ & & & $1.974 E-02$ & & & $5.632 E-03$ \\
\hline Iron (III) Oxalate & & & & & & $2.529 \mathrm{E}+01$ & & & $9.862 \mathrm{E}+00$ \\
\hline Iron (III) Citrate & & & & & & $4.876 \mathrm{E}+01$ & & & $1.391 \mathrm{E}+01$ \\
\hline Iron (III) Hydrogen EDTA & & $1.511 \mathrm{E}-02$ & $7.205 \mathrm{E}+00$ & & & $4.105 \mathrm{E}+01$ & & & $1.171 \mathrm{E}+01$ \\
\hline Iron (III) Hydroxide & $8.320 \mathrm{E}+01$ & & & & & $3.706 \mathrm{E}+01$ & & & $3.022 \mathrm{E}+00$ \\
\hline \multicolumn{10}{|l|}{ Formic Acid, dimer } \\
\hline \multicolumn{10}{|l|}{ Hydrofluoride, dimer } \\
\hline 2,2'-Iminobisacetic acid & & $5.129 \mathrm{E}-02$ & $2.446 \mathrm{E}+01$ & & & $2.446 \mathrm{E}+01$ & & & $6.979 \mathrm{E}+00$ \\
\hline Water & & $8.614 \mathrm{E}+01$ & $4.109 \mathrm{E}+04$ & $7.780 \mathrm{E}+02$ & $7.619 \mathrm{E}+03$ & $4.880 \mathrm{E}+04$ & $9.968 \mathrm{E}+02$ & $7.123 \mathrm{E}+03$ & $1.593 \mathrm{E}+04$ \\
\hline \multicolumn{10}{|l|}{ Sulfuric Acid } \\
\hline Hydrogen Chloride & & $4.656 \mathrm{E}-01$ & $2.221 \mathrm{E}+02$ & & & $2.342 \mathrm{E}+02$ & & & $6.680 \mathrm{E}+01$ \\
\hline Formic Acid & & $1.794 \mathrm{E}-02$ & $8.557 \mathrm{E}+00$ & & & $8.557 \mathrm{E}+00$ & & & $2.441 \mathrm{E}+00$ \\
\hline Hydrofluoric Acid & & $1.385 \mathrm{E}-01$ & $6.605 \mathrm{E}+01$ & & & $5.709 \mathrm{E}+01$ & & & $1.451 \mathrm{E}+01$ \\
\hline \multicolumn{10}{|l|}{ Mercury (II) Chloride } \\
\hline Mercury (elemental) & & $2.626 E-08$ & $1.252 E-05$ & & & $1.252 E-05$ & & & $3.572 E-06$ \\
\hline Mercury (II) Oxide & $2.864 E+01$ & $1.824 E-03$ & $8.701 E-01$ & & & $2.951 E+01$ & & & $9.502 E-01$ \\
\hline Nitrous (III) Acid & & $4.206 \mathrm{E}+00$ & $2.006 \mathrm{E}+03$ & & & $3.077 \mathrm{E}+03$ & & & $8.777 \mathrm{E}+02$ \\
\hline Nitric Acid & & $2.888 \mathrm{E}+00$ & $1.377 \mathrm{E}+03$ & & & $1.377 \mathrm{E}+03$ & & & $3.929 \mathrm{E}+02$ \\
\hline Hexachlorobutadiene & & $2.403 E-10$ & $1.146 E-07$ & & & $1.146 E-07$ & & & $3.270 E-08$ \\
\hline Potassium Citrate & & $1.279 \mathrm{E}-01$ & $6.100 \mathrm{E}+01$ & & & & & & \\
\hline \multicolumn{10}{|l|}{ Potassium Acetate } \\
\hline Potassium Ch loride & & $1.692 \mathrm{E}+00$ & $8.070 \mathrm{E}+02$ & & & $7.783 \mathrm{E}+02$ & & & $2.220 \mathrm{E}+02$ \\
\hline
\end{tabular}


WSRC-TR-2002-00550, Rev. 1

SRT-RPP-2002-0027.

\begin{tabular}{|c|c|c|c|c|c|c|c|c|c|}
\hline Stream & $\begin{array}{l}\text { FCW5 } \\
\text { Solids }\end{array}$ & $\begin{array}{l}\text { FCW5 } \\
\text { Recycle }\end{array}$ & $\begin{array}{l}\text { FCW5 } \\
\text { Return }\end{array}$ & Leach-in & Total Leach & $\begin{array}{l}\text { FCWash- } \\
\text { Leach }\end{array}$ & Steam-in & Total Steam & UF2 Filtrate \\
\hline Potassium Glycolate & & & & & & $1.121 \mathrm{E}+02$ & & & $3.197 \mathrm{E}+01$ \\
\hline \multicolumn{10}{|l|}{ Potassium Nitrate (VI) } \\
\hline gamma-BHC (Lindane) & & $6.692 E-07$ & $3.192 E-04$ & & & $3.192 E-04$ & & & $9.106 E-05$ \\
\hline $\begin{array}{c}\text { 4-methyl-2-pentanone } \\
\text { (MIBK) }\end{array}$ & & $1.278 E-07$ & $6.095 E-05$ & & & $6.095 E-05$ & & & $1.739 E-05$ \\
\hline Magnesium Oxalate & & $7.285 \mathrm{E}-07$ & $3.475 \mathrm{E}-04$ & & & & & & \\
\hline Magnesium Dichromate (VI) & & & & & & $2.469 \mathrm{E}-06$ & & & 9.791E-07 \\
\hline Magnesium Hydroxide & $2.385 \mathrm{E}+02$ & & & & & $2.385 \mathrm{E}+02$ & & & \\
\hline Manganese (II) & & & & & & $2.341 \mathrm{E}-02$ & & & $6.679 \mathrm{E}-03$ \\
\hline \multicolumn{10}{|l|}{ Orthophosphate } \\
\hline Manganese (II) Oxalate & & $5.934 \mathrm{E}-05$ & $2.831 \mathrm{E}-02$ & & & & & & \\
\hline \multicolumn{10}{|l|}{ Manganese (II) Hydroxide } \\
\hline Nitrogen & & $2.635 \mathrm{E}-08$ & $1.257 \mathrm{E}-05$ & & & $1.257 \mathrm{E}-05$ & & & $3.585 \mathrm{E}-06$ \\
\hline Sodium Oxalate & $8.184 \mathrm{E}+03$ & $2.503 \mathrm{E}-01$ & $1.194 \mathrm{E}+02$ & & & $8.307 \mathrm{E}+03$ & & & \\
\hline Sodium Chromate (VI) & & $3.782 \mathrm{E}-01$ & $1.804 \mathrm{E}+02$ & & & $1.445 \mathrm{E}+02$ & & & $4.091 \mathrm{E}+01$ \\
\hline Sodium Fluoride Sulfate & & & & & & $1.604 \mathrm{E}+03$ & & & \\
\hline Sodium Acetate & & $8.312 \mathrm{E}-03$ & $3.965 \mathrm{E}+00$ & & & & & & \\
\hline Sodium Glycolate & & $2.064 \mathrm{E}-01$ & $9.844 \mathrm{E}+01$ & & & & & & \\
\hline Sodium Nitrite & & $3.293 \mathrm{E}+00$ & $1.571 \mathrm{E}+03$ & & & & & & \\
\hline Sodium Hydroxide & & $1.584 \mathrm{E}+01$ & $7.558 \mathrm{E}+03$ & $7.599 \mathrm{E}+02$ & $7.442 \mathrm{E}+03$ & $1.498 \mathrm{E}+04$ & & & $4.411 \mathrm{E}+03$ \\
\hline Naphthalene & & $3.389 \mathrm{E}-08$ & $1.616 \mathrm{E}-05$ & & & $1.616 \mathrm{E}-05$ & & & $4.611 \mathrm{E}-06$ \\
\hline Sodium Aluminosilicate Gel & $3.690 \mathrm{E}+03$ & & & & & $3.348 \mathrm{E}+03$ & & & \\
\hline Nickel Orthophosphate & & & & & & $7.098 \mathrm{E}-02$ & & & $2.025 \mathrm{E}-02$ \\
\hline Nickel (II) Oxalate & & $1.789 \mathrm{E}-04$ & $8.535 \mathrm{E}-02$ & & & & & & \\
\hline \multicolumn{10}{|l|}{ Nickel (II) Hydroxide } \\
\hline Oxygen & & $1.466 \mathrm{E}-08$ & $6.993 \mathrm{E}-06$ & & & $6.993 \mathrm{E}-06$ & & & $1.995 \mathrm{E}-06$ \\
\hline Phosphorus Pentoxide & & $2.895 \mathrm{E}-02$ & $1.381 \mathrm{E}+01$ & & & $1.377 \mathrm{E}+01$ & & & $3.929 \mathrm{E}+00$ \\
\hline PYRENE & & $1.052 \mathrm{E}-06$ & $5.020 \mathrm{E}-04$ & & & $5.020 \mathrm{E}-04$ & & & $1.432 \mathrm{E}-04$ \\
\hline Silicon Dioxide & & $3.961 \mathrm{E}-03$ & $1.889 \mathrm{E}+00$ & & & $1.258 \mathrm{E}+02$ & & & $3.044 \mathrm{E}+01$ \\
\hline Sulfur Trioxide & & $2.151 \mathrm{E}+00$ & $1.026 \mathrm{E}+03$ & & & $3.282 \mathrm{E}+02$ & & & $1.861 \mathrm{E}+02$ \\
\hline 1,2,4-trichlorobenzene & & $7.155 \mathrm{E}-09$ & $3.413 \mathrm{E}-06$ & & & $3.413 \mathrm{E}-06$ & & & $9.736 \mathrm{E}-07$ \\
\hline 1,2,3-trichloropropane & & 5.043E-08 & $2.406 \mathrm{E}-05$ & & & $2.406 \mathrm{E}-05$ & & & $6.862 \mathrm{E}-06$ \\
\hline TOLUENE & & 4.443E-09 & $2.119 \mathrm{E}-06$ & & & $2.119 \mathrm{E}-06$ & & & $6.046 \mathrm{E}-07$ \\
\hline Zinc Dichloride Pentoxide & & & & & & $1.453 \mathrm{E}+01$ & & & $4.144 \mathrm{E}+00$ \\
\hline Zinc Oxalate & & $5.160 \mathrm{E}-02$ & $2.461 \mathrm{E}+01$ & & & & & & \\
\hline \multicolumn{10}{|l|}{ Zinc Hydroxide } \\
\hline Zirconium EDTA & & $6.862 \mathrm{E}-04$ & $3.273 \mathrm{E}-01$ & & & & & & \\
\hline Zirconium Oxide & & & & & & $1.063 \mathrm{E}-01$ & & & 3.032E-02 \\
\hline Total $\mathrm{g} / \mathrm{hr}$ & $1.455 \mathrm{E}+04$ & $1.218 \mathrm{E}+02$ & $5.810 \mathrm{E}+04$ & $1.538 \mathrm{E}+03$ & $1.506 \mathrm{E}+04$ & $8.771 \mathrm{E}+04$ & $9.968 \mathrm{E}+02$ & $7.123 \mathrm{E}+03$ & $2.295 \mathrm{E}+04$ \\
\hline Volume, L/hr & $3.741 \mathrm{E}+00$ & $1.000 \mathrm{E}-01$ & $4.770 \mathrm{E}+01$ & $1.001 \mathrm{E}+00$ & $9.801 \mathrm{E}+00$ & $6.020 \mathrm{E}+01$ & $1.000 \mathrm{E}+00$ & $7.146 \mathrm{E}+00$ & $1.832 \mathrm{E}+01$ \\
\hline Enthalpy, cal/hr & $-4.284 \mathrm{E}+07$ & $-4.027 \mathrm{E}+05$ & $-1.921 \mathrm{E}+08$ & $-4.956 \mathrm{E}+06$ & $-4.854 \mathrm{E}+07$ & $-2.840 \mathrm{E}+08$ & $-3.780 \mathrm{E}+06$ & $-2.701 \mathrm{E}+07$ & $-7.708 \mathrm{E}+07$ \\
\hline \multicolumn{10}{|l|}{ Vapor fraction } \\
\hline Solid fraction & $1.000 \mathrm{E}+00$ & & & & & $2.920 \mathrm{E}-02$ & & & \\
\hline \multicolumn{10}{|l|}{ Organic fraction } \\
\hline Osmotic Pres, atm & & $1.722 \mathrm{E}+02$ & $1.722 \mathrm{E}+02$ & $4.623 \mathrm{E}+03$ & $4.623 \mathrm{E}+03$ & $4.195 \mathrm{E}+02$ & & & $3.703 \mathrm{E}+02$ \\
\hline \multicolumn{10}{|l|}{ Redox Pot, volts } \\
\hline E-Con, 1/ohm-cm & & $2.001 \mathrm{E}-01$ & $2.001 \mathrm{E}-01$ & & & $2.602 \mathrm{E}-01$ & 5.489E-08 & 5.489E-08 & \\
\hline E-Con, cm2/ohm-mol & & $2.114 \mathrm{E}+01$ & $2.114 \mathrm{E}+01$ & & & $2.326 \mathrm{E}+01$ & & & \\
\hline Abs Visc, cP & & $2.303 \mathrm{E}+00$ & $2.303 \mathrm{E}+00$ & $4.904 \mathrm{E}+01$ & $4.904 \mathrm{E}+01$ & $4.276 \mathrm{E}+00$ & $8.907 \mathrm{E}-01$ & $8.907 \mathrm{E}-01$ & \\
\hline Rel Visc & & $2.585 \mathrm{E}+00$ & $2.585 \mathrm{E}+00$ & $5.506 \mathrm{E}+01$ & $5.506 \mathrm{E}+01$ & $4.801 \mathrm{E}+00$ & $1.000 \mathrm{E}+00$ & $1.000 \mathrm{E}+00$ & \\
\hline Ionic Strength & & $5.508 \mathrm{E}+00$ & $5.508 \mathrm{E}+00$ & $2.442 \mathrm{E}+01$ & $2.442 \mathrm{E}+01$ & $7.781 \mathrm{E}+00$ & $1.008 \mathrm{E}-07$ & $1.008 \mathrm{E}-07$ & $7.029 \mathrm{E}+00$ \\
\hline
\end{tabular}


WSRC-TR-2002-00550, Rev. 1

SRT-RPP-2002-0027.

Table B- 1. Waste Feed Evaporator 5 Stage Model Results for UF 1.22 Density Case (cont'd) ${ }^{8}$

\begin{tabular}{|c|c|c|c|c|c|c|c|c|c|}
\hline Stream & UF2 Slurry & $\begin{array}{l}\text { SCWash1 } \\
\text { Slurry }\end{array}$ & $\begin{array}{l}\text { SCW1 } \\
\text { Liquor }\end{array}$ & $\begin{array}{l}\text { SCW1 } \\
\text { Solids }\end{array}$ & $\begin{array}{c}\text { SCW1 } \\
\text { Recycle }\end{array}$ & $\begin{array}{l}\text { SCW1 } \\
\text { Return }\end{array}$ & $\begin{array}{l}\text { SCW2 } \\
\text { Slurry }\end{array}$ & $\begin{array}{l}\text { SCW2 } \\
\text { Liquor }\end{array}$ & $\begin{array}{l}\text { SCW2 } \\
\text { Solids }\end{array}$ \\
\hline Phase & Mixed & Mixed & Aqueous & Solid & Aqueous & Aqueous & Mixed & Aqueous & Solid \\
\hline Temperature, $\mathrm{C}$ & 25 & 25 & 25 & 25 & 25 & 25 & 25 & 25 & 25 \\
\hline Pressure, atm & 1 & 1 & 1 & 1 & 1 & 1 & 1 & 1 & 1 \\
\hline $\mathrm{pH}$ & 14.8733 & 14.8715 & 14.8715 & & 14.8715 & 14.8715 & 14.8696 & 14.8696 & \\
\hline Total $\mathrm{mol} / \mathrm{hr}$ & 2703.34 & 2709 & 2615.48 & 93.5186 & 5.68446 & 2609.8 & 2708.98 & 2615.53 & 93.4496 \\
\hline Flow Units & $\mathrm{g} / \mathrm{hr}$ & $\mathrm{g} / \mathrm{hr}$ & $\mathrm{g} / \mathrm{hr}$ & $\mathrm{g} / \mathrm{hr}$ & $\mathrm{g} / \mathrm{hr}$ & $\mathrm{g} / \mathrm{hr}$ & $\mathrm{g} / \mathrm{hr}$ & $\mathrm{g} / \mathrm{hr}$ & $\mathrm{g} / \mathrm{hr}$ \\
\hline \multicolumn{10}{|l|}{ Acetic Acid, Dimer } \\
\hline Acetic Acid & $3.027 \mathrm{E}+00$ & $3.027 \mathrm{E}+00$ & $3.027 \mathrm{E}+00$ & & $6.580 \mathrm{E}-03$ & $3.021 \mathrm{E}+00$ & $3.021 \mathrm{E}+00$ & $3.021 \mathrm{E}+00$ & \\
\hline Aluminum Oxide & $4.874 \mathrm{E}+02$ & $4.873 \mathrm{E}+02$ & $4.873 \mathrm{E}+02$ & & $1.059 \mathrm{E}+00$ & $4.862 \mathrm{E}+02$ & $4.861 \mathrm{E}+02$ & $4.861 \mathrm{E}+02$ & \\
\hline Aldrin & $3.694 E-04$ & $3.694 E-04$ & $3.694 E-04$ & & $8.028 E-07$ & $3.686 E-04$ & $3.686 E-04$ & $3.686 E-04$ & \\
\hline \multicolumn{10}{|l|}{ Aluminum Hydrogen EDTA } \\
\hline \multicolumn{10}{|l|}{ Aluminum Hydroxide } \\
\hline Benzene & $1.822 E-06$ & $1.822 E-06$ & $1.822 E-06$ & & $3.961 E-09$ & $1.818 E-06$ & $1.818 E-06$ & $1.818 E-06$ & \\
\hline $\begin{array}{c}\text { Bis(2-ethylhexyl)phthalate } \\
\text { (BEHP) }\end{array}$ & $4.642 E-03$ & $4.642 E-03$ & $4.642 E-03$ & & $1.009 E-05$ & $4.632 E-03$ & $4.632 E-03$ & $4.632 E-03$ & \\
\hline Benzo(a)pyrene (BaP) & $4.180 E+00$ & $4.180 E+00$ & $2.153 E-05$ & $4.180 E+00$ & $4.679 E-08$ & $2.148 E-05$ & $4.180 E+00$ & $2.158 E-05$ & $4.180 E+00$ \\
\hline Phenol & $6.469 E-02$ & $6.469 E-02$ & $6.469 E-02$ & & $1.406 E-04$ & $6.455 E-02$ & $6.455 E-02$ & $6.455 E-02$ & \\
\hline \multicolumn{10}{|l|}{ Calcium Chloride Oxide } \\
\hline \multicolumn{10}{|l|}{ Calcium Oxalate } \\
\hline \multicolumn{10}{|l|}{ Calcium Oxalate Monohydrate } \\
\hline \multicolumn{10}{|l|}{ Calcium Carbonate } \\
\hline Calcium Dichromate (VI) & $2.088 \mathrm{E}+01$ & $2.089 \mathrm{E}+01$ & $2.089 \mathrm{E}+01$ & & $4.541 \mathrm{E}-02$ & $2.085 \mathrm{E}+01$ & $2.086 \mathrm{E}+01$ & $2.086 \mathrm{E}+01$ & \\
\hline Calcium Fluoride & $1.384 \mathrm{E}+03$ & $1.387 \mathrm{E}+03$ & & $1.387 \mathrm{E}+03$ & & & $1.389 \mathrm{E}+03$ & & $1.389 \mathrm{E}+03$ \\
\hline Calcium Hydroxide & $1.414 \mathrm{E}+02$ & $1.388 \mathrm{E}+02$ & & $1.388 \mathrm{E}+02$ & & & $1.361 \mathrm{E}+02$ & & $1.361 \mathrm{E}+02$ \\
\hline Pentachlorophenol & $5.586 E-03$ & $5.586 E-03$ & $5.586 E-03$ & & $1.214 E-05$ & $5.574 E-03$ & $5.574 E-03$ & $5.574 E-03$ & \\
\hline Hexachlorobenzene & $4.183 E+00$ & $4.183 E+00$ & $5.139 E-05$ & $4.183 E+00$ & $1.117 E-07$ & $5.128 E-05$ & $4.183 E+00$ & $5.152 E-05$ & $4.183 E+00$ \\
\hline Chlorobenzene & $3.909 E-06$ & $3.909 E-06$ & $3.909 E-06$ & & $8.495 E-09$ & $3.900 E-06$ & $3.900 E-06$ & $3.900 E-06$ & \\
\hline 2-Chloronaphthalene & $1.050 E-05$ & $1.050 E-05$ & $1.050 E-05$ & & $2.282 E-08$ & $1.048 E-05$ & $1.048 E-05$ & $1.048 E-05$ & \\
\hline Carbon Dioxide & $1.212 \mathrm{E}+03$ & $1.212 \mathrm{E}+03$ & $1.212 \mathrm{E}+03$ & & $2.634 \mathrm{E}+00$ & $1.209 \mathrm{E}+03$ & $1.209 \mathrm{E}+03$ & $1.209 \mathrm{E}+03$ & \\
\hline \multicolumn{10}{|l|}{ Cesium Acetate } \\
\hline Cesium Glycolate & $3.300 \mathrm{E}+00$ & $3.300 \mathrm{E}+00$ & $3.300 \mathrm{E}+00$ & & $7.171 \mathrm{E}-03$ & $3.292 \mathrm{E}+00$ & $3.292 \mathrm{E}+00$ & $3.292 \mathrm{E}+00$ & \\
\hline Dibenz[a,h]anthracene & $2.595 E-03$ & $2.595 E-03$ & $2.595 E-03$ & & $5.640 E-06$ & $2.589 E-03$ & $2.589 E-03$ & $2.589 E-03$ & \\
\hline 1,2-dibromoethane & $9.394 E-05$ & $9.394 E-05$ & $9.394 E-05$ & & $2.042 E-07$ & $9.373 E-05$ & $9.373 E-05$ & $9.373 E-05$ & \\
\hline Diethyl phthalate & $1.411 E-02$ & $1.411 E-02$ & $1.411 E-02$ & & $3.067 E-05$ & $1.408 E-02$ & $1.408 E-02$ & $1.408 E-02$ & \\
\hline Iron (III) Oxalate & $2.471 \mathrm{E}+01$ & $2.485 \mathrm{E}+01$ & $2.485 \mathrm{E}+01$ & & $5.400 \mathrm{E}-02$ & $2.479 \mathrm{E}+01$ & $2.493 \mathrm{E}+01$ & $2.493 \mathrm{E}+01$ & \\
\hline Iron (III) Citrate & $3.485 \mathrm{E}+01$ & $3.485 \mathrm{E}+01$ & $3.485 \mathrm{E}+01$ & & $7.575 \mathrm{E}-02$ & $3.478 \mathrm{E}+01$ & $3.478 \mathrm{E}+01$ & $3.478 \mathrm{E}+01$ & \\
\hline Iron (III) Hydrogen EDTA & $2.934 \mathrm{E}+01$ & $2.934 \mathrm{E}+01$ & $2.934 \mathrm{E}+01$ & & $6.376 \mathrm{E}-02$ & $2.928 \mathrm{E}+01$ & $2.928 \mathrm{E}+01$ & $2.928 \mathrm{E}+01$ & \\
\hline Iron (III) Hydroxide & $2.876 \mathrm{E}+01$ & $2.868 \mathrm{E}+01$ & $7.427 \mathrm{E}+00$ & $2.126 \mathrm{E}+01$ & $1.614 \mathrm{E}-02$ & $7.411 \mathrm{E}+00$ & $2.859 \mathrm{E}+01$ & $7.265 \mathrm{E}+00$ & $2.133 \mathrm{E}+01$ \\
\hline \multicolumn{10}{|l|}{ Formic Acid, dimer } \\
\hline \multicolumn{10}{|l|}{ Hydrofluoride, dimer } \\
\hline 2,2'-Iminobisacetic acid & $1.749 \mathrm{E}+01$ & $1.749 \mathrm{E}+01$ & $1.749 \mathrm{E}+01$ & & $3.800 \mathrm{E}-02$ & $1.745 \mathrm{E}+01$ & $1.745 \mathrm{E}+01$ & $1.745 \mathrm{E}+01$ & \\
\hline Water & $3.991 \mathrm{E}+04$ & $4.001 \mathrm{E}+04$ & $4.001 \mathrm{E}+04$ & & $8.696 \mathrm{E}+01$ & $3.992 \mathrm{E}+04$ & $4.002 \mathrm{E}+04$ & $4.002 \mathrm{E}+04$ & \\
\hline \multicolumn{10}{|l|}{ Sulfuric Acid } \\
\hline Hydrogen Chloride & $1.674 \mathrm{E}+02$ & $1.674 \mathrm{E}+02$ & $1.674 \mathrm{E}+02$ & & $3.638 \mathrm{E}-01$ & $1.670 \mathrm{E}+02$ & $1.670 \mathrm{E}+02$ & $1.670 \mathrm{E}+02$ & \\
\hline Formic Acid & $6.116 \mathrm{E}+00$ & $6.116 \mathrm{E}+00$ & $6.116 \mathrm{E}+00$ & & $1.329 \mathrm{E}-02$ & $6.103 \mathrm{E}+00$ & $6.103 \mathrm{E}+00$ & $6.103 \mathrm{E}+00$ & \\
\hline Hydrofluoric Acid & $3.635 \mathrm{E}+01$ & $3.629 \mathrm{E}+01$ & $3.629 \mathrm{E}+01$ & & $7.886 \mathrm{E}-02$ & $3.621 \mathrm{E}+01$ & $3.615 \mathrm{E}+01$ & $3.615 \mathrm{E}+01$ & \\
\hline \multicolumn{10}{|l|}{ Mercury (II) Chloride } \\
\hline Mercury (elemental) & $8.951 E-06$ & $8.951 E-06$ & $8.951 E-06$ & & $1.945 E-08$ & $8.932 E-06$ & $8.932 E-06$ & $8.932 E-06$ & \\
\hline Mercury (II) Oxide & $2.856 E+01$ & $2.856 E+01$ & $2.381 E+00$ & $2.617 E+01$ & $5.174 E-03$ & $2.375 E+00$ & $2.855 E+01$ & $2.375 E+00$ & $2.617 E+01$ \\
\hline Nitrous (III) Acid & $2.199 \mathrm{E}+03$ & $2.199 \mathrm{E}+03$ & $2.199 \mathrm{E}+03$ & & $4.780 \mathrm{E}+00$ & $2.194 \mathrm{E}+03$ & $2.194 \mathrm{E}+03$ & $2.194 \mathrm{E}+03$ & \\
\hline Nitric Acid & $9.845 \mathrm{E}+02$ & $9.845 \mathrm{E}+02$ & $9.845 \mathrm{E}+02$ & & $2.140 \mathrm{E}+00$ & $9.823 \mathrm{E}+02$ & $9.823 \mathrm{E}+02$ & $9.823 \mathrm{E}+02$ & \\
\hline Hexachlorobutadiene & $8.194 E-08$ & $8.194 E-08$ & $8.194 E-08$ & & $1.781 E-10$ & $8.176 E-08$ & $8.176 E-08$ & $8.176 E-08$ & \\
\hline \multicolumn{10}{|l|}{ Potassium Citrate } \\
\hline \multicolumn{10}{|l|}{ Potassium Acetate } \\
\hline Potassium Chloride & $5.563 \mathrm{E}+02$ & $5.563 \mathrm{E}+02$ & $5.563 \mathrm{E}+02$ & & $1.209 \mathrm{E}+00$ & $5.551 \mathrm{E}+02$ & $5.551 \mathrm{E}+02$ & $5.551 \mathrm{E}+02$ & \\
\hline
\end{tabular}


WSRC-TR-2002-00550, Rev. 1 SRT-RPP-2002-0027.

\begin{tabular}{|c|c|c|c|c|c|c|c|c|c|}
\hline Stream & UF2 Slurry & $\begin{array}{l}\text { SCWash1 } \\
\text { Slurry }\end{array}$ & $\begin{array}{l}\text { SCW1 } \\
\text { Liquor }\end{array}$ & $\begin{array}{l}\text { SCW1 } \\
\text { Solids }\end{array}$ & $\begin{array}{c}\text { SCW1 } \\
\text { Recycle }\end{array}$ & $\begin{array}{l}\text { SCW1 } \\
\text { Return }\end{array}$ & $\begin{array}{l}\text { SCW2 } \\
\text { Slurry }\end{array}$ & $\begin{array}{l}\text { SCW2 } \\
\text { Liquor }\end{array}$ & $\begin{array}{l}\text { SCW2 } \\
\text { Solids }\end{array}$ \\
\hline Potassium Glycolate & $8.011 \mathrm{E}+01$ & $8.011 \mathrm{E}+01$ & $8.011 \mathrm{E}+01$ & & $1.741 \mathrm{E}-01$ & $7.994 \mathrm{E}+01$ & $7.994 \mathrm{E}+01$ & $7.994 \mathrm{E}+01$ & \\
\hline \multicolumn{10}{|l|}{ Potassium Nitrate (VI) } \\
\hline gamma-BHC (Lindane) & $2.282 E-04$ & $2.282 E-04$ & $2.282 E-04$ & & $4.959 E-07$ & $2.277 E-04$ & $2.277 E-04$ & $2.277 E-04$ & \\
\hline $\begin{array}{l}\text { 4-methyl-2-pentanone } \\
\text { (MIBK) }\end{array}$ & $4.357 E-05$ & $4.357 E-05$ & $4.357 E-05$ & & $9.469 E-08$ & $4.347 E-05$ & $4.347 E-05$ & $4.347 E-05$ & \\
\hline \multicolumn{10}{|l|}{ Magnesium Oxalate } \\
\hline Magnesium Dichromate (VI) & $2.453 \mathrm{E}-06$ & $2.468 \mathrm{E}-06$ & $2.468 \mathrm{E}-06$ & & $5.363 \mathrm{E}-09$ & $2.462 \mathrm{E}-06$ & $2.477 \mathrm{E}-06$ & $2.477 \mathrm{E}-06$ & \\
\hline Magnesium Hydroxide & $2.385 \mathrm{E}+02$ & $2.385 \mathrm{E}+02$ & & $2.385 \mathrm{E}+02$ & & & $2.385 \mathrm{E}+02$ & & $2.385 \mathrm{E}+02$ \\
\hline $\begin{array}{c}\text { Manganese (II) } \\
\text { Orthophosphate }\end{array}$ & $1.674 \mathrm{E}-02$ & $1.674 \mathrm{E}-02$ & $1.674 \mathrm{E}-02$ & & $3.637 \mathrm{E}-05$ & $1.670 \mathrm{E}-02$ & $1.670 \mathrm{E}-02$ & $1.670 \mathrm{E}-02$ & \\
\hline \multicolumn{10}{|l|}{ Manganese (II) Oxalate } \\
\hline \multicolumn{10}{|l|}{ Manganese (II) Hydroxide } \\
\hline Nitrogen & 8.984E-06 & $8.984 \mathrm{E}-06$ & $8.984 \mathrm{E}-06$ & & $1.952 \mathrm{E}-08$ & $8.964 \mathrm{E}-06$ & $8.964 \mathrm{E}-06$ & $8.964 \mathrm{E}-06$ & \\
\hline Sodium Oxalate & $8.297 \mathrm{E}+03$ & $8.297 \mathrm{E}+03$ & & $8.297 \mathrm{E}+03$ & & & $8.297 \mathrm{E}+03$ & & $8.297 \mathrm{E}+03$ \\
\hline Sodium Chromate (VI) & $1.025 \mathrm{E}+02$ & $1.025 \mathrm{E}+02$ & $1.025 \mathrm{E}+02$ & & $2.228 \mathrm{E}-01$ & $1.023 \mathrm{E}+02$ & $1.023 \mathrm{E}+02$ & $1.023 \mathrm{E}+02$ & \\
\hline Sodium Fluoride Sulfate & $8.589 \mathrm{E}+02$ & $8.463 \mathrm{E}+02$ & & $8.463 \mathrm{E}+02$ & & & $8.336 \mathrm{E}+02$ & & $8.336 \mathrm{E}+02$ \\
\hline \multicolumn{10}{|l|}{ Sodium Acetate } \\
\hline \multicolumn{10}{|l|}{ Sodium Glycolate } \\
\hline \multicolumn{10}{|l|}{ Sodium Nitrite } \\
\hline Sodium Hydroxide & $1.105 \mathrm{E}+04$ & $1.106 \mathrm{E}+04$ & $1.106 \mathrm{E}+04$ & & $2.404 \mathrm{E}+01$ & $1.104 \mathrm{E}+04$ & $1.105 \mathrm{E}+04$ & $1.105 \mathrm{E}+04$ & \\
\hline Naphthalene & $1.155 \mathrm{E}-05$ & $1.155 \mathrm{E}-05$ & $1.155 \mathrm{E}-05$ & & $2.511 \mathrm{E}-08$ & $1.153 \mathrm{E}-05$ & $1.153 \mathrm{E}-05$ & $1.153 \mathrm{E}-05$ & \\
\hline Sodium Aluminosilicate Gel & $3.401 \mathrm{E}+03$ & $3.401 \mathrm{E}+03$ & & $3.401 \mathrm{E}+03$ & & & $3.402 \mathrm{E}+03$ & & $3.402 \mathrm{E}+03$ \\
\hline Nickel Orthophosphate & $5.073 \mathrm{E}-02$ & $5.073 \mathrm{E}-02$ & $5.073 \mathrm{E}-02$ & & $1.103 \mathrm{E}-04$ & $5.062 \mathrm{E}-02$ & $5.062 \mathrm{E}-02$ & $5.062 \mathrm{E}-02$ & \\
\hline \multicolumn{10}{|l|}{ Nickel (II) Oxalate } \\
\hline \multicolumn{10}{|l|}{ Nickel (II) Hydroxide } \\
\hline Oxygen & $4.998 \mathrm{E}-06$ & $4.998 \mathrm{E}-06$ & $4.998 \mathrm{E}-06$ & & $1.086 \mathrm{E}-08$ & $4.987 \mathrm{E}-06$ & $4.987 \mathrm{E}-06$ & $4.987 \mathrm{E}-06$ & \\
\hline Phosphorus Pentoxide & $9.844 \mathrm{E}+00$ & $9.844 \mathrm{E}+00$ & $9.844 \mathrm{E}+00$ & & $2.139 \mathrm{E}-02$ & $9.822 \mathrm{E}+00$ & $9.822 \mathrm{E}+00$ & $9.822 \mathrm{E}+00$ & \\
\hline PYRENE & $3.588 \mathrm{E}-04$ & $3.588 \mathrm{E}-04$ & $3.588 \mathrm{E}-04$ & & 7.798E-07 & $3.580 \mathrm{E}-04$ & $3.580 \mathrm{E}-04$ & $3.580 \mathrm{E}-04$ & \\
\hline Silicon Dioxide & $7.628 \mathrm{E}+01$ & $7.610 \mathrm{E}+01$ & $7.610 \mathrm{E}+01$ & & $1.654 \mathrm{E}-01$ & $7.593 \mathrm{E}+01$ & $7.575 \mathrm{E}+01$ & $7.575 \mathrm{E}+01$ & \\
\hline Sulfur Trioxide & $4.662 \mathrm{E}+02$ & $4.717 \mathrm{E}+02$ & $4.717 \mathrm{E}+02$ & & $1.025 \mathrm{E}+00$ & $4.707 \mathrm{E}+02$ & $4.762 \mathrm{E}+02$ & $4.762 \mathrm{E}+02$ & \\
\hline 1,2,4-trichlorobenzene & $2.439 \mathrm{E}-06$ & $2.439 \mathrm{E}-06$ & $2.439 \mathrm{E}-06$ & & 5.302E-09 & $2.434 \mathrm{E}-06$ & $2.434 \mathrm{E}-06$ & $2.434 \mathrm{E}-06$ & \\
\hline 1,2,3-trichloropropane & $1.719 \mathrm{E}-05$ & $1.719 \mathrm{E}-05$ & $1.719 \mathrm{E}-05$ & & $3.737 \mathrm{E}-08$ & $1.716 \mathrm{E}-05$ & $1.716 \mathrm{E}-05$ & $1.716 \mathrm{E}-05$ & \\
\hline TOLUENE & $1.515 \mathrm{E}-06$ & $1.515 \mathrm{E}-06$ & $1.515 \mathrm{E}-06$ & & 3.292E-09 & $1.512 \mathrm{E}-06$ & $1.512 \mathrm{E}-06$ & $1.512 \mathrm{E}-06$ & \\
\hline Zinc Dichloride Pentoxide & $1.038 \mathrm{E}+01$ & $1.038 \mathrm{E}+01$ & $1.038 \mathrm{E}+01$ & & $2.257 \mathrm{E}-02$ & $1.036 \mathrm{E}+01$ & $1.036 \mathrm{E}+01$ & $1.036 \mathrm{E}+01$ & \\
\hline \multicolumn{10}{|l|}{ Zinc Oxalate } \\
\hline \multicolumn{10}{|l|}{ Zinc Hydroxide } \\
\hline \multicolumn{10}{|l|}{ Zirconium EDTA } \\
\hline Zirconium Oxide & $7.598 \mathrm{E}-02$ & $7.598 \mathrm{E}-02$ & $7.598 \mathrm{E}-02$ & & $1.651 \mathrm{E}-04$ & $7.581 \mathrm{E}-02$ & $7.581 \mathrm{E}-02$ & $7.581 \mathrm{E}-02$ & \\
\hline Total $\mathrm{g} / \mathrm{hr}$ & $7.188 \mathrm{E}+04$ & $7.198 \mathrm{E}+04$ & $5.762 \mathrm{E}+04$ & $1.436 \mathrm{E}+04$ & $1.252 \mathrm{E}+02$ & $5.749 \mathrm{E}+04$ & $7.195 \mathrm{E}+04$ & $5.760 \mathrm{E}+04$ & $1.435 \mathrm{E}+04$ \\
\hline Volume, L/hr & $4.911 \mathrm{E}+01$ & $4.921 \mathrm{E}+01$ & $4.601 \mathrm{E}+01$ & $3.198 \mathrm{E}+00$ & $1.000 \mathrm{E}-01$ & $4.591 \mathrm{E}+01$ & $4.921 \mathrm{E}+01$ & $4.601 \mathrm{E}+01$ & $3.198 \mathrm{E}+00$ \\
\hline Enthalpy, cal/hr & $-2.340 \mathrm{E}+08$ & $-2.344 \mathrm{E}+08$ & $-1.935 \mathrm{E}+08$ & $-4.087 \mathrm{E}+07$ & $-4.206 \mathrm{E}+05$ & $-1.931 \mathrm{E}+08$ & $-2.344 \mathrm{E}+08$ & $-1.935 \mathrm{E}+08$ & $-4.084 \mathrm{E}+07$ \\
\hline \multicolumn{10}{|l|}{ Vapor fraction } \\
\hline Solid fraction & $3.462 \mathrm{E}-02$ & $3.452 \mathrm{E}-02$ & & $1.000 \mathrm{E}+00$ & & & $3.450 \mathrm{E}-02$ & & $1.000 \mathrm{E}+00$ \\
\hline \multicolumn{10}{|l|}{ Organic fraction } \\
\hline Osmotic Pres, atm & $3.703 \mathrm{E}+02$ & $3.695 \mathrm{E}+02$ & $3.695 \mathrm{E}+02$ & & $3.695 \mathrm{E}+02$ & $3.695 \mathrm{E}+02$ & $3.687 \mathrm{E}+02$ & $3.687 \mathrm{E}+02$ & \\
\hline \multicolumn{10}{|l|}{ Redox Pot, volts } \\
\hline E-Con, 1/ohm-cm & & $2.592 \mathrm{E}-01$ & $2.592 \mathrm{E}-01$ & & $2.592 \mathrm{E}-01$ & $2.592 \mathrm{E}-01$ & $2.592 \mathrm{E}-01$ & $2.592 \mathrm{E}-01$ & \\
\hline E-Con, cm2/ohm-mol & & $2.445 \mathrm{E}+01$ & $2.445 \mathrm{E}+01$ & & $2.445 \mathrm{E}+01$ & $2.445 \mathrm{E}+01$ & $2.448 \mathrm{E}+01$ & $2.448 \mathrm{E}+01$ & \\
\hline Abs Visc, cP & & $3.701 \mathrm{E}+00$ & $3.701 \mathrm{E}+00$ & & $3.701 \mathrm{E}+00$ & $3.701 \mathrm{E}+00$ & $3.692 \mathrm{E}+00$ & $3.692 \mathrm{E}+00$ & \\
\hline Rel Visc & & $4.155 \mathrm{E}+00$ & $4.155 \mathrm{E}+00$ & & $4.155 \mathrm{E}+00$ & $4.155 \mathrm{E}+00$ & $4.145 \mathrm{E}+00$ & $4.145 \mathrm{E}+00$ & \\
\hline Ionic Strength & $7.029 \mathrm{E}+00$ & $7.017 \mathrm{E}+00$ & $7.017 \mathrm{E}+00$ & & $7.017 \mathrm{E}+00$ & $7.017 \mathrm{E}+00$ & $7.005 \mathrm{E}+00$ & $7.005 \mathrm{E}+00$ & \\
\hline
\end{tabular}


WSRC-TR-2002-00550, Rev. 1

SRT-RPP-2002-0027.

Table B- 1. Waste Feed Evaporator 5 Stage Model Results for UF 1.22 Density Case (cont'd) ${ }^{8}$

\begin{tabular}{|c|c|c|c|c|c|c|c|c|c|}
\hline Stream & $\begin{array}{c}\text { SCW2 } \\
\text { Recycle }\end{array}$ & $\begin{array}{l}\text { SCW2 } \\
\text { Return }\end{array}$ & $\begin{array}{l}\text { SCW3 } \\
\text { Slurry }\end{array}$ & $\begin{array}{l}\text { SCW3 } \\
\text { Liquor }\end{array}$ & $\begin{array}{l}\text { SCW3 } \\
\text { Solids }\end{array}$ & $\begin{array}{c}\text { SCW3 } \\
\text { Recycle }\end{array}$ & $\begin{array}{l}\text { SCW3 } \\
\text { Return }\end{array}$ & $\begin{array}{l}\text { SCW4 } \\
\text { Slurry }\end{array}$ & $\begin{array}{l}\text { SCW4 } \\
\text { Liquor }\end{array}$ \\
\hline Phase & Aqueous & Aqueous & Mixed & Aqueous & Solid & Aqueous & Aqueous & Mixed & Aqueous \\
\hline Temperature, $\mathrm{C}$ & 25 & 25 & 25 & 25 & 25 & 25 & 25 & 25 & 25 \\
\hline Pressure, atm & 1 & 1 & 1 & 1 & 1 & 1 & 1 & 1 & 1 \\
\hline $\mathrm{pH}$ & 14.8696 & 14.8696 & 14.8678 & 14.8678 & & 14.8678 & 14.8678 & 14.866 & 14.866 \\
\hline Total mol/hr & 5.68434 & 2609.84 & 2708.95 & 2615.57 & 93.3801 & 5.68422 & 2609.89 & 2708.93 & 2615.62 \\
\hline Flow Units & $\mathrm{g} / \mathrm{hr}$ & $\mathrm{g} / \mathrm{hr}$ & $\mathrm{g} / \mathrm{hr}$ & $\mathrm{g} / \mathrm{hr}$ & $\mathrm{g} / \mathrm{hr}$ & $\mathrm{g} / \mathrm{hr}$ & $\mathrm{g} / \mathrm{hr}$ & $\mathrm{g} / \mathrm{hr}$ & $\mathrm{g} / \mathrm{hr}$ \\
\hline \multicolumn{10}{|l|}{ Acetic Acid, Dimer } \\
\hline Acetic Acid & $6.565 \mathrm{E}-03$ & $3.014 \mathrm{E}+00$ & $3.014 \mathrm{E}+00$ & $3.014 \mathrm{E}+00$ & & $6.551 \mathrm{E}-03$ & $3.008 \mathrm{E}+00$ & $3.008 \mathrm{E}+00$ & $3.008 \mathrm{E}+00$ \\
\hline Aluminum Oxide & $1.056 \mathrm{E}+00$ & $4.850 \mathrm{E}+02$ & $4.849 \mathrm{E}+02$ & $4.849 \mathrm{E}+02$ & & $1.054 \mathrm{E}+00$ & $4.839 \mathrm{E}+02$ & $4.837 \mathrm{E}+02$ & $4.837 \mathrm{E}+02$ \\
\hline Aldrin & $8.010 E-07$ & $3.678 E-04$ & $3.678 E-04$ & $3.678 E-04$ & & $7.992 E-07$ & $3.670 E-04$ & $3.670 E-04$ & $3.670 E-04$ \\
\hline \multicolumn{10}{|l|}{ Aluminum Hydrogen EDTA } \\
\hline \multicolumn{10}{|l|}{ Aluminum Hydroxide } \\
\hline Benzene & $3.952 E-09$ & $1.814 E-06$ & $1.814 E-06$ & $1.814 E-06$ & & $3.943 E-09$ & $1.811 E-06$ & $1.811 E-06$ & $1.811 E-06$ \\
\hline $\begin{array}{c}\text { Bis(2-ethylhexyl)phthalate } \\
\text { (BEHP) }\end{array}$ & $1.007 E-05$ & $4.622 E-03$ & $4.622 E-03$ & $4.622 E-03$ & & $1.004 E-05$ & $4.612 E-03$ & $4.612 E-03$ & $4.612 E-03$ \\
\hline Benzo(a)pyrene (BaP) & $4.691 E-08$ & $2.154 E-05$ & $4.180 E+00$ & $2.164 E-05$ & $4.180 E+00$ & $4.702 E-08$ & $2.159 E-05$ & $4.180 E+00$ & $2.169 E-05$ \\
\hline Phenol & $1.403 E-04$ & $6.441 E-02$ & $6.441 E-02$ & $6.441 E-02$ & & $1.400 E-04$ & $6.427 E-02$ & $6.427 E-02$ & $6.427 E-02$ \\
\hline \multicolumn{10}{|l|}{ Calcium Chloride Oxide } \\
\hline \multicolumn{10}{|l|}{ Calcium Oxalate } \\
\hline \multicolumn{10}{|l|}{ Calcium Oxalate Monohydrate } \\
\hline \multicolumn{10}{|l|}{ Calcium Carbonate } \\
\hline Calcium Dichromate (VI) & $4.533 \mathrm{E}-02$ & $2.081 \mathrm{E}+01$ & $2.082 \mathrm{E}+01$ & $2.082 \mathrm{E}+01$ & & $4.525 \mathrm{E}-02$ & $2.078 \mathrm{E}+01$ & $2.079 \mathrm{E}+01$ & $2.079 \mathrm{E}+01$ \\
\hline Calcium Fluoride & & & $1.392 \mathrm{E}+03$ & & $1.392 \mathrm{E}+03$ & & & $1.395 \mathrm{E}+03$ & \\
\hline Calcium Hydroxide & & & $1.334 \mathrm{E}+02$ & & $1.334 \mathrm{E}+02$ & & & $1.307 \mathrm{E}+02$ & \\
\hline Pentachlorophenol & $1.211 E-05$ & $5.562 E-03$ & $5.562 E-03$ & $5.562 E-03$ & & $1.209 E-05$ & $5.550 E-03$ & $5.550 E-03$ & $5.550 E-03$ \\
\hline Hexachlorobenzene & $1.120 E-07$ & $5.141 E-05$ & $4.183 E+00$ & $5.165 E-05$ & $4.183 E+00$ & $1.123 E-07$ & $5.154 E-05$ & $4.183 E+00$ & $5.178 E-05$ \\
\hline Chlorobenzene & $8.476 E-09$ & $3.892 E-06$ & $3.892 E-06$ & $3.892 E-06$ & & $8.458 E-09$ & $3.883 E-06$ & $3.883 E-06$ & $3.883 E-06$ \\
\hline 2-Chloronaphthalene & $2.277 E-08$ & $1.045 E-05$ & $1.045 E-05$ & $1.045 E-05$ & & $2.272 E-08$ & $1.043 E-05$ & $1.043 E-05$ & $1.043 E-05$ \\
\hline Carbon Dioxide & $2.628 \mathrm{E}+00$ & $1.207 \mathrm{E}+03$ & $1.207 \mathrm{E}+03$ & $1.207 \mathrm{E}+03$ & & $2.622 \mathrm{E}+00$ & $1.204 \mathrm{E}+03$ & $1.204 \mathrm{E}+03$ & $1.204 \mathrm{E}+03$ \\
\hline \multicolumn{10}{|l|}{ Cesium Acetate } \\
\hline Cesium Glycolate & $7.155 \mathrm{E}-03$ & $3.285 \mathrm{E}+00$ & $3.285 \mathrm{E}+00$ & $3.285 \mathrm{E}+00$ & & $7.139 \mathrm{E}-03$ & $3.278 \mathrm{E}+00$ & $3.278 \mathrm{E}+00$ & $3.278 \mathrm{E}+00$ \\
\hline Dibenz[a,h]anthracene & $5.627 E-06$ & $2.584 E-03$ & $2.584 E-03$ & $2.584 E-03$ & & $5.615 E-06$ & $2.578 E-03$ & $2.578 E-03$ & $2.578 E-03$ \\
\hline 1,2-dibromoethane & $2.037 E-07$ & $9.353 E-05$ & $9.353 E-05$ & $9.353 E-05$ & & $2.033 E-07$ & $9.333 E-05$ & $9.332 E-05$ & $9.332 E-05$ \\
\hline Diethyl phthalate & $3.060 E-05$ & $1.405 E-02$ & $1.405 E-02$ & $1.405 E-02$ & & $3.053 E-05$ & $1.402 E-02$ & $1.402 E-02$ & $1.402 E-02$ \\
\hline Iron (III) Oxalate & $5.417 \mathrm{E}-02$ & $2.487 \mathrm{E}+01$ & $2.501 \mathrm{E}+01$ & $2.501 \mathrm{E}+01$ & & $5.435 \mathrm{E}-02$ & $2.495 \mathrm{E}+01$ & $2.509 \mathrm{E}+01$ & $2.509 \mathrm{E}+01$ \\
\hline Iron (III) Citrate & $7.558 \mathrm{E}-02$ & $3.470 \mathrm{E}+01$ & $3.470 \mathrm{E}+01$ & $3.470 \mathrm{E}+01$ & & $7.542 \mathrm{E}-02$ & $3.463 \mathrm{E}+01$ & $3.463 \mathrm{E}+01$ & $3.463 \mathrm{E}+01$ \\
\hline Iron (III) Hydrogen EDTA & $6.362 \mathrm{E}-02$ & $2.921 \mathrm{E}+01$ & $2.921 \mathrm{E}+01$ & $2.921 \mathrm{E}+01$ & & $6.348 \mathrm{E}-02$ & $2.915 \mathrm{E}+01$ & $2.915 \mathrm{E}+01$ & $2.915 \mathrm{E}+01$ \\
\hline Iron (III) Hydroxide & $1.579 \mathrm{E}-02$ & $7.249 \mathrm{E}+00$ & $2.850 \mathrm{E}+01$ & $7.104 \mathrm{E}+00$ & $2.139 \mathrm{E}+01$ & $1.544 \mathrm{E}-02$ & $7.089 \mathrm{E}+00$ & $2.841 \mathrm{E}+01$ & $6.945 \mathrm{E}+00$ \\
\hline \multicolumn{10}{|l|}{ Formic Acid, dimer } \\
\hline \multicolumn{10}{|l|}{ Hydrofluoride, dimer } \\
\hline 2,2'-Iminobisacetic acid & $3.792 \mathrm{E}-02$ & $1.741 \mathrm{E}+01$ & $1.741 \mathrm{E}+01$ & $1.741 \mathrm{E}+01$ & & $3.784 \mathrm{E}-02$ & $1.737 \mathrm{E}+01$ & $1.737 \mathrm{E}+01$ & $1.737 \mathrm{E}+01$ \\
\hline Water & $8.698 \mathrm{E}+01$ & $3.994 \mathrm{E}+04$ & $4.003 \mathrm{E}+04$ & $4.003 \mathrm{E}+04$ & & $8.700 \mathrm{E}+01$ & $3.995 \mathrm{E}+04$ & $4.005 \mathrm{E}+04$ & $4.005 \mathrm{E}+04$ \\
\hline \multicolumn{10}{|l|}{ Sulfuric Acid } \\
\hline Hydrogen Chloride & $3.630 \mathrm{E}-01$ & $1.667 \mathrm{E}+02$ & $1.667 \mathrm{E}+02$ & $1.667 \mathrm{E}+02$ & & $3.622 \mathrm{E}-01$ & $1.663 \mathrm{E}+02$ & $1.663 \mathrm{E}+02$ & $1.663 \mathrm{E}+02$ \\
\hline Formic Acid & $1.326 \mathrm{E}-02$ & $6.090 \mathrm{E}+00$ & $6.089 \mathrm{E}+00$ & $6.089 \mathrm{E}+00$ & & $1.323 \mathrm{E}-02$ & $6.076 \mathrm{E}+00$ & $6.076 \mathrm{E}+00$ & $6.076 \mathrm{E}+00$ \\
\hline Hydrofluoric Acid & $7.856 \mathrm{E}-02$ & $3.607 \mathrm{E}+01$ & $3.601 \mathrm{E}+01$ & $3.601 \mathrm{E}+01$ & & $7.825 \mathrm{E}-02$ & $3.593 \mathrm{E}+01$ & $3.587 \mathrm{E}+01$ & $3.587 \mathrm{E}+01$ \\
\hline \multicolumn{10}{|l|}{ Mercury (II) Chloride } \\
\hline Mercury (elemental) & $1.941 E-08$ & $8.913 E-06$ & $8.913 E-06$ & $8.913 E-06$ & & $1.937 E-08$ & $8.893 E-06$ & $8.893 E-06$ & $8.893 E-06$ \\
\hline Mercury (II) Oxide & $5.162 E-03$ & $2.370 E+00$ & $2.854 E+01$ & $2.370 E+00$ & $2.618 E+01$ & $5.150 E-03$ & $2.364 E+00$ & $2.854 E+01$ & $2.364 E+00$ \\
\hline Nitrous (III) Acid & $4.769 \mathrm{E}+00$ & $2.190 \mathrm{E}+03$ & $2.190 \mathrm{E}+03$ & $2.190 \mathrm{E}+03$ & & $4.758 \mathrm{E}+00$ & $2.185 \mathrm{E}+03$ & $2.185 \mathrm{E}+03$ & $2.185 \mathrm{E}+03$ \\
\hline Nitric Acid & $2.135 \mathrm{E}+00$ & $9.802 \mathrm{E}+02$ & $9.802 \mathrm{E}+02$ & $9.802 \mathrm{E}+02$ & & $2.130 \mathrm{E}+00$ & $9.781 \mathrm{E}+02$ & $9.781 \mathrm{E}+02$ & $9.781 \mathrm{E}+02$ \\
\hline Hexachlorobutadiene & $1.777 E-10$ & $8.158 E-08$ & $8.158 E-08$ & $8.158 E-08$ & & $1.773 E-10$ & $8.141 E-08$ & $8.141 E-08$ & $8.141 E-08$ \\
\hline \multicolumn{10}{|l|}{ Potassium Citrate } \\
\hline \multicolumn{10}{|l|}{ Potassium Acetate } \\
\hline Potassium Chloride & $1.206 \mathrm{E}+00$ & $5.539 \mathrm{E}+02$ & $5.539 \mathrm{E}+02$ & $5.539 \mathrm{E}+02$ & & $1.204 \mathrm{E}+00$ & $5.527 \mathrm{E}+02$ & $5.527 \mathrm{E}+02$ & $5.527 \mathrm{E}+02$ \\
\hline
\end{tabular}


WSRC-TR-2002-00550, Rev. 1

SRT-RPP-2002-0027.

\begin{tabular}{|c|c|c|c|c|c|c|c|c|c|}
\hline Stream & $\begin{array}{c}\text { SCW2 } \\
\text { Recycle }\end{array}$ & $\begin{array}{l}\text { SCW2 } \\
\text { Return }\end{array}$ & $\begin{array}{l}\text { SCW3 } \\
\text { Slurry }\end{array}$ & $\begin{array}{l}\text { SCW3 } \\
\text { Liquor }\end{array}$ & $\begin{array}{l}\text { SCW3 } \\
\text { Solids }\end{array}$ & $\begin{array}{c}\text { SCW3 } \\
\text { Recycle }\end{array}$ & $\begin{array}{l}\text { SCW3 } \\
\text { Return }\end{array}$ & $\begin{array}{l}\text { SCW4 } \\
\text { Slurry }\end{array}$ & $\begin{array}{l}\text { SCW4 } \\
\text { Liquor }\end{array}$ \\
\hline Potassium Glycolate & $1.737 \mathrm{E}-01$ & $7.977 \mathrm{E}+01$ & $7.977 \mathrm{E}+01$ & $7.977 \mathrm{E}+01$ & & $1.733 \mathrm{E}-01$ & $7.959 \mathrm{E}+01$ & $7.959 \mathrm{E}+01$ & $7.959 \mathrm{E}+01$ \\
\hline \multicolumn{10}{|l|}{ Potassium Nitrate (VI) } \\
\hline gamma-BHC (Lindane) & $4.948 E-07$ & $2.272 E-04$ & $2.272 E-04$ & $2.272 E-04$ & & $4.937 E-07$ & $2.267 E-04$ & $2.267 E-04$ & $2.267 E-04$ \\
\hline $\begin{array}{c}\text { 4-methyl-2-pentanone } \\
(\mathrm{MIBK})\end{array}$ & $9.448 E-08$ & $4.338 E-05$ & $4.338 E-05$ & $4.338 E-05$ & & $9.427 E-08$ & $4.328 E-05$ & $4.328 E-05$ & $4.328 E-05$ \\
\hline \multicolumn{10}{|l|}{ Magnesium Oxalate } \\
\hline Magnesium Dichromate (VI) & 5.383E-09 & $2.471 \mathrm{E}-06$ & $2.486 \mathrm{E}-06$ & $2.486 \mathrm{E}-06$ & & $5.402 \mathrm{E}-09$ & $2.481 \mathrm{E}-06$ & $2.495 \mathrm{E}-06$ & $2.495 \mathrm{E}-06$ \\
\hline Magnesium Hydroxide & & & $2.385 \mathrm{E}+02$ & & $2.385 \mathrm{E}+02$ & & & $2.385 \mathrm{E}+02$ & \\
\hline $\begin{array}{l}\text { Manganese (II) } \\
\text { Orthophosphate }\end{array}$ & $3.629 \mathrm{E}-05$ & $1.666 \mathrm{E}-02$ & $1.666 \mathrm{E}-02$ & $1.666 \mathrm{E}-02$ & & $3.621 \mathrm{E}-05$ & $1.663 \mathrm{E}-02$ & $1.663 \mathrm{E}-02$ & $1.663 \mathrm{E}-02$ \\
\hline \multicolumn{10}{|l|}{ Manganese (II) Oxalate } \\
\hline \multicolumn{10}{|l|}{ Manganese (II) Hydroxide } \\
\hline Nitrogen & $1.948 \mathrm{E}-08$ & $8.945 \mathrm{E}-06$ & $8.945 \mathrm{E}-06$ & $8.945 \mathrm{E}-06$ & & $1.944 \mathrm{E}-08$ & $8.925 \mathrm{E}-06$ & $8.925 \mathrm{E}-06$ & $8.925 \mathrm{E}-06$ \\
\hline Sodium Oxalate & & & $8.297 \mathrm{E}+03$ & & $8.297 \mathrm{E}+03$ & & & $8.297 \mathrm{E}+03$ & \\
\hline Sodium Chromate (VI) & $2.223 \mathrm{E}-01$ & $1.020 \mathrm{E}+02$ & $1.020 \mathrm{E}+02$ & $1.020 \mathrm{E}+02$ & & $2.217 \mathrm{E}-01$ & $1.018 \mathrm{E}+02$ & $1.018 \mathrm{E}+02$ & $1.018 \mathrm{E}+02$ \\
\hline Sodium Fluoride Sulfate & & & $8.208 \mathrm{E}+02$ & & $8.208 \mathrm{E}+02$ & & & $8.080 \mathrm{E}+02$ & \\
\hline \multicolumn{10}{|l|}{ Sodium Acetate } \\
\hline \multicolumn{10}{|l|}{ Sodium Glycolate } \\
\hline \multicolumn{10}{|l|}{ Sodium Nitrite } \\
\hline Sodium Hydroxide & $2.401 \mathrm{E}+01$ & $1.102 \mathrm{E}+04$ & $1.103 \mathrm{E}+04$ & $1.103 \mathrm{E}+04$ & & $2.397 \mathrm{E}+01$ & $1.101 \mathrm{E}+04$ & $1.101 \mathrm{E}+04$ & $1.101 \mathrm{E}+04$ \\
\hline Naphthalene & $2.506 \mathrm{E}-08$ & $1.150 \mathrm{E}-05$ & $1.150 \mathrm{E}-05$ & $1.150 \mathrm{E}-05$ & & $2.500 \mathrm{E}-08$ & $1.148 \mathrm{E}-05$ & $1.148 \mathrm{E}-05$ & $1.148 \mathrm{E}-05$ \\
\hline Sodium Aluminosilicate Gel & & & $3.402 \mathrm{E}+03$ & & $3.402 \mathrm{E}+03$ & & & $3.403 \mathrm{E}+03$ & \\
\hline Nickel Orthophosphate & $1.100 \mathrm{E}-04$ & $5.051 \mathrm{E}-02$ & $5.051 \mathrm{E}-02$ & $5.051 \mathrm{E}-02$ & & $1.098 \mathrm{E}-04$ & $5.040 \mathrm{E}-02$ & $5.040 \mathrm{E}-02$ & $5.040 \mathrm{E}-02$ \\
\hline \multicolumn{10}{|l|}{ Nickel (II) Oxalate } \\
\hline \multicolumn{10}{|l|}{ Nickel (II) Hydroxide } \\
\hline Oxygen & $1.084 \mathrm{E}-08$ & $4.977 \mathrm{E}-06$ & $4.977 \mathrm{E}-06$ & $4.977 \mathrm{E}-06$ & & $1.082 \mathrm{E}-08$ & $4.966 \mathrm{E}-06$ & $4.966 \mathrm{E}-06$ & $4.966 \mathrm{E}-06$ \\
\hline Phosphorus Pentoxide & $2.135 \mathrm{E}-02$ & $9.801 \mathrm{E}+00$ & $9.801 \mathrm{E}+00$ & $9.801 \mathrm{E}+00$ & & $2.130 \mathrm{E}-02$ & $9.780 \mathrm{E}+00$ & $9.780 \mathrm{E}+00$ & $9.780 \mathrm{E}+00$ \\
\hline PYRENE & $7.781 \mathrm{E}-07$ & $3.572 \mathrm{E}-04$ & $3.572 \mathrm{E}-04$ & $3.572 \mathrm{E}-04$ & & $7.764 \mathrm{E}-07$ & $3.565 \mathrm{E}-04$ & $3.565 \mathrm{E}-04$ & $3.565 \mathrm{E}-04$ \\
\hline Silicon Dioxide & $1.646 \mathrm{E}-01$ & $7.559 \mathrm{E}+01$ & $7.541 \mathrm{E}+01$ & $7.541 \mathrm{E}+01$ & & $1.639 \mathrm{E}-01$ & $7.524 \mathrm{E}+01$ & $7.506 \mathrm{E}+01$ & $7.506 \mathrm{E}+01$ \\
\hline Sulfur Trioxide & $1.035 \mathrm{E}+00$ & $4.752 \mathrm{E}+02$ & $4.807 \mathrm{E}+02$ & $4.807 \mathrm{E}+02$ & & $1.045 \mathrm{E}+00$ & $4.797 \mathrm{E}+02$ & $4.853 \mathrm{E}+02$ & $4.853 \mathrm{E}+02$ \\
\hline 1,2,4-trichlorobenzene & $5.290 \mathrm{E}-09$ & $2.429 \mathrm{E}-06$ & $2.429 \mathrm{E}-06$ & $2.429 \mathrm{E}-06$ & & $5.278 \mathrm{E}-09$ & $2.424 \mathrm{E}-06$ & $2.424 \mathrm{E}-06$ & $2.424 \mathrm{E}-06$ \\
\hline 1,2,3-trichloropropane & $3.729 \mathrm{E}-08$ & $1.712 \mathrm{E}-05$ & $1.712 \mathrm{E}-05$ & $1.712 \mathrm{E}-05$ & & $3.721 \mathrm{E}-08$ & $1.708 \mathrm{E}-05$ & $1.708 \mathrm{E}-05$ & $1.708 \mathrm{E}-05$ \\
\hline TOLUENE & $3.285 \mathrm{E}-09$ & $1.508 \mathrm{E}-06$ & $1.508 \mathrm{E}-06$ & $1.508 \mathrm{E}-06$ & & $3.278 \mathrm{E}-09$ & $1.505 \mathrm{E}-06$ & $1.505 \mathrm{E}-06$ & $1.505 \mathrm{E}-06$ \\
\hline Zinc Dichloride Pentoxide & $2.252 \mathrm{E}-02$ & $1.034 \mathrm{E}+01$ & $1.034 \mathrm{E}+01$ & $1.034 \mathrm{E}+01$ & & $2.247 \mathrm{E}-02$ & $1.032 \mathrm{E}+01$ & $1.032 \mathrm{E}+01$ & $1.032 \mathrm{E}+01$ \\
\hline \multicolumn{10}{|l|}{ Zinc Oxalate } \\
\hline \multicolumn{10}{|l|}{ Zinc Hydroxide } \\
\hline \multicolumn{10}{|l|}{ Zirconium EDTA } \\
\hline Zirconium Oxide & $1.648 \mathrm{E}-04$ & $7.565 \mathrm{E}-02$ & $7.565 \mathrm{E}-02$ & $7.565 \mathrm{E}-02$ & & $1.644 \mathrm{E}-04$ & $7.548 \mathrm{E}-02$ & $7.548 \mathrm{E}-02$ & $7.548 \mathrm{E}-02$ \\
\hline Total g/hr & $1.252 \mathrm{E}+02$ & $5.748 \mathrm{E}+04$ & $7.193 \mathrm{E}+04$ & $5.759 \mathrm{E}+04$ & $1.434 \mathrm{E}+04$ & $1.252 \mathrm{E}+02$ & $5.746 \mathrm{E}+04$ & $7.190 \mathrm{E}+04$ & $5.758 \mathrm{E}+04$ \\
\hline Volume, L/hr & $1.000 \mathrm{E}-01$ & $4.591 \mathrm{E}+01$ & $4.921 \mathrm{E}+01$ & $4.601 \mathrm{E}+01$ & $3.198 \mathrm{E}+00$ & $1.000 \mathrm{E}-01$ & $4.591 \mathrm{E}+01$ & $4.921 \mathrm{E}+01$ & $4.602 \mathrm{E}+01$ \\
\hline Enthalpy, cal/hr & $-4.206 \mathrm{E}+05$ & $-1.931 \mathrm{E}+08$ & $-2.343 \mathrm{E}+08$ & $-1.935 \mathrm{E}+08$ & $-4.081 \mathrm{E}+07$ & $-4.206 \mathrm{E}+05$ & $-1.931 \mathrm{E}+08$ & $-2.343 \mathrm{E}+08$ & $-1.935 \mathrm{E}+08$ \\
\hline \multicolumn{10}{|l|}{ Vapor fraction } \\
\hline Solid fraction & & & $3.447 \mathrm{E}-02$ & & $1.000 \mathrm{E}+00$ & & & $3.445 \mathrm{E}-02$ & \\
\hline \multicolumn{10}{|l|}{ Organic fraction } \\
\hline Osmotic Pres, atm & $3.687 \mathrm{E}+02$ & $3.687 \mathrm{E}+02$ & $3.679 \mathrm{E}+02$ & $3.679 \mathrm{E}+02$ & & $3.679 \mathrm{E}+02$ & $3.679 \mathrm{E}+02$ & $3.672 \mathrm{E}+02$ & $3.672 \mathrm{E}+02$ \\
\hline \multicolumn{10}{|l|}{ Redox Pot, volts } \\
\hline E-Con, 1/ohm-cm & $2.592 \mathrm{E}-01$ & $2.592 \mathrm{E}-01$ & $2.591 \mathrm{E}-01$ & $2.591 \mathrm{E}-01$ & & $2.591 \mathrm{E}-01$ & $2.591 \mathrm{E}-01$ & $2.591 \mathrm{E}-01$ & $2.591 \mathrm{E}-01$ \\
\hline E-Con, cm2/ohm-mol & $2.448 \mathrm{E}+01$ & $2.448 \mathrm{E}+01$ & $2.451 \mathrm{E}+01$ & $2.451 \mathrm{E}+01$ & & $2.451 \mathrm{E}+01$ & $2.451 \mathrm{E}+01$ & $2.454 \mathrm{E}+01$ & $2.454 \mathrm{E}+01$ \\
\hline Abs Visc, cP & $3.692 \mathrm{E}+00$ & $3.692 \mathrm{E}+00$ & $3.684 \mathrm{E}+00$ & $3.684 \mathrm{E}+00$ & & $3.684 \mathrm{E}+00$ & $3.684 \mathrm{E}+00$ & $3.675 \mathrm{E}+00$ & $3.675 \mathrm{E}+00$ \\
\hline Rel Visc & $4.145 \mathrm{E}+00$ & $4.145 \mathrm{E}+00$ & $4.136 \mathrm{E}+00$ & $4.136 \mathrm{E}+00$ & & $4.136 \mathrm{E}+00$ & $4.136 \mathrm{E}+00$ & $4.126 \mathrm{E}+00$ & $4.126 \mathrm{E}+00$ \\
\hline Ionic Strength & $7.005 \mathrm{E}+00$ & $7.005 \mathrm{E}+00$ & $6.993 \mathrm{E}+00$ & $6.993 \mathrm{E}+00$ & & $6.993 \mathrm{E}+00$ & $6.993 \mathrm{E}+00$ & $6.982 \mathrm{E}+00$ & $6.982 \mathrm{E}+00$ \\
\hline
\end{tabular}


WSRC-TR-2002-00550, Rev. 1

SRT-RPP-2002-0027.

Table B- 1. Waste Feed Evaporator 5 Stage Model Results for UF 1.22 Density Case (cont'd) ${ }^{8}$

\begin{tabular}{|c|c|c|c|c|c|c|c|c|c|}
\hline Stream & $\begin{array}{l}\text { SCW4 } \\
\text { Solids }\end{array}$ & $\begin{array}{c}\text { SCW4 } \\
\text { Recycle }\end{array}$ & $\begin{array}{l}\text { SCW4 } \\
\text { Return }\end{array}$ & $\begin{array}{c}\text { SCWash5 } \\
\text { Slurry }\end{array}$ & $\begin{array}{l}\text { SCW5 } \\
\text { Liquor }\end{array}$ & $\begin{array}{l}\text { SCW5 } \\
\text { Solids }\end{array}$ & $\begin{array}{c}\text { SCW5 } \\
\text { Recycle }\end{array}$ & $\begin{array}{l}\text { SCW5 } \\
\text { Return }\end{array}$ & Acid Rinse \\
\hline Phase & Solid & Aqueous & Aqueous & Mixed & Aqueous & Solid & Aqueous & Aqueous & Aqueous \\
\hline Temperature, $\mathrm{C}$ & 25 & 25 & 25 & 25 & 25 & 25 & 25 & 25 & 25 \\
\hline Pressure, atm & 1 & 1 & 1 & 1 & 1 & 1 & 1 & 1 & 1 \\
\hline $\mathrm{pH}$ & & 14.866 & 14.866 & 14.8642 & 14.8642 & & 14.8642 & 14.8642 & -0.301785 \\
\hline Total mol/hr & 93.3101 & 5.6841 & 2609.94 & 2708.91 & 2615.67 & 93.2397 & 5.68398 & 2609.99 & 53.9076 \\
\hline Flow Units & $\mathrm{g} / \mathrm{hr}$ & $\mathrm{g} / \mathrm{hr}$ & $\mathrm{g} / \mathrm{hr}$ & $\mathrm{g} / \mathrm{hr}$ & $\mathrm{g} / \mathrm{hr}$ & $\mathrm{g} / \mathrm{hr}$ & $\mathrm{g} / \mathrm{hr}$ & $\mathrm{g} / \mathrm{hr}$ & $\mathrm{g} / \mathrm{hr}$ \\
\hline \multicolumn{10}{|l|}{ Acetic Acid, Dimer } \\
\hline Acetic Acid & & $6.536 \mathrm{E}-03$ & $3.001 \mathrm{E}+00$ & $3.001 \mathrm{E}+00$ & $3.001 \mathrm{E}+00$ & & $6.522 \mathrm{E}-03$ & $2.995 \mathrm{E}+00$ & \\
\hline Aluminum Oxide & & $1.051 \mathrm{E}+00$ & $4.827 \mathrm{E}+02$ & $4.825 \mathrm{E}+02$ & $4.825 \mathrm{E}+02$ & & $1.049 \mathrm{E}+00$ & $4.815 \mathrm{E}+02$ & $1.214 \mathrm{E}+00$ \\
\hline Aldrin & & $7.975 E-07$ & $3.662 E-04$ & $3.662 E-04$ & $3.662 E-04$ & & $7.957 E-07$ & $3.654 E-04$ & \\
\hline \multicolumn{10}{|l|}{ Aluminum Hydrogen EDTA } \\
\hline \multicolumn{10}{|l|}{ Aluminum Hydroxide } \\
\hline Benzene & & $3.935 E-09$ & $1.807 E-06$ & $1.807 E-06$ & $1.807 E-06$ & & $3.926 E-09$ & $1.803 E-06$ & \\
\hline $\begin{array}{c}\text { Bis(2-ethylhexyl)phthalate } \\
\text { (BEHP) }\end{array}$ & & $1.002 E-05$ & $4.601 E-03$ & $4.601 E-03$ & $4.601 E-03$ & & $9.999 E-06$ & $4.591 E-03$ & \\
\hline Benzo(a)pyrene (BaP) & $4.180 E+00$ & $4.714 E-08$ & $2.165 E-05$ & $4.180 E+00$ & $2.175 E-05$ & $4.180 E+00$ & $4.726 E-08$ & $2.170 E-05$ & \\
\hline Phenol & & $1.397 E-04$ & $6.413 E-02$ & $6.413 E-02$ & $6.413 E-02$ & & $1.394 E-04$ & $6.399 E-02$ & \\
\hline \multicolumn{10}{|l|}{ Calcium Chloride Oxide } \\
\hline \multicolumn{10}{|l|}{ Calcium Oxalate } \\
\hline \multicolumn{10}{|l|}{ Calcium Oxalate Monohydrate } \\
\hline \multicolumn{10}{|l|}{ Calcium Carbonate } \\
\hline Calcium Dichromate (VI) & & $4.517 \mathrm{E}-02$ & $2.074 \mathrm{E}+01$ & $2.075 \mathrm{E}+01$ & $2.075 \mathrm{E}+01$ & & $4.509 \mathrm{E}-02$ & $2.070 \mathrm{E}+01$ & \\
\hline Calcium Fluoride & $1.395 \mathrm{E}+03$ & & & $1.398 \mathrm{E}+03$ & & $1.398 \mathrm{E}+03$ & & & \\
\hline Calcium Hydroxide & $1.307 \mathrm{E}+02$ & & & $1.280 \mathrm{E}+02$ & & $1.280 \mathrm{E}+02$ & & & \\
\hline Pentachlorophenol & & $1.206 E-05$ & $5.538 E-03$ & $5.538 E-03$ & $5.538 E-03$ & & $1.203 E-05$ & $5.526 E-03$ & \\
\hline Hexachlorobenzene & $4.183 E+00$ & $1.125 E-07$ & $5.167 E-05$ & $4.183 E+00$ & $5.191 E-05$ & $4.183 E+00$ & $1.128 E-07$ & $5.180 E-05$ & \\
\hline Chlorobenzene & & $8.439 E-09$ & $3.875 E-06$ & $3.875 E-06$ & $3.875 E-06$ & & $8.420 E-09$ & $3.866 E-06$ & \\
\hline 2-Chloronaphthalene & & $2.267 E-08$ & $1.041 E-05$ & $1.041 E-05$ & $1.041 E-05$ & & $2.262 E-08$ & $1.039 E-05$ & \\
\hline Carbon Dioxide & & $2.616 \mathrm{E}+00$ & $1.201 \mathrm{E}+03$ & $1.201 \mathrm{E}+03$ & $1.201 \mathrm{E}+03$ & & $2.611 \mathrm{E}+00$ & $1.199 \mathrm{E}+03$ & \\
\hline \multicolumn{10}{|l|}{ Cesium Acetate } \\
\hline Cesium Glycolate & & $7.124 \mathrm{E}-03$ & $3.271 \mathrm{E}+00$ & $3.271 \mathrm{E}+00$ & $3.271 \mathrm{E}+00$ & & $7.108 \mathrm{E}-03$ & $3.264 \mathrm{E}+00$ & \\
\hline Dibenz[a,h]anthracene & & $5.602 E-06$ & $2.572 E-03$ & $2.572 E-03$ & $2.572 E-03$ & & $5.590 E-06$ & $2.567 E-03$ & \\
\hline 1,2-dibromoethane & & $2.028 E-07$ & $9.312 E-05$ & $9.312 E-05$ & $9.312 E-05$ & & $2.024 E-07$ & $9.292 E-05$ & \\
\hline Diethyl phthalate & & $3.047 E-05$ & $1.399 E-02$ & $1.399 E-02$ & $1.399 E-02$ & & $3.040 E-05$ & $1.396 E-02$ & \\
\hline Iron (III) Oxalate & & $5.452 \mathrm{E}-02$ & $2.503 \mathrm{E}+01$ & $2.517 \mathrm{E}+01$ & $2.517 \mathrm{E}+01$ & & $5.469 \mathrm{E}-02$ & $2.511 \mathrm{E}+01$ & \\
\hline Iron (III) Citrate & & $7.525 \mathrm{E}-02$ & $3.455 \mathrm{E}+01$ & $3.455 \mathrm{E}+01$ & $3.455 \mathrm{E}+01$ & & $7.508 \mathrm{E}-02$ & $3.448 \mathrm{E}+01$ & \\
\hline Iron (III) Hydrogen EDTA & & $6.334 \mathrm{E}-02$ & $2.908 \mathrm{E}+01$ & $2.908 \mathrm{E}+01$ & $2.908 \mathrm{E}+01$ & & $6.320 \mathrm{E}-02$ & $2.902 \mathrm{E}+01$ & \\
\hline Iron (III) Hydroxide & $2.146 \mathrm{E}+01$ & $1.509 \mathrm{E}-02$ & $6.930 \mathrm{E}+00$ & $2.831 \mathrm{E}+01$ & $6.786 \mathrm{E}+00$ & $2.153 \mathrm{E}+01$ & $1.475 \mathrm{E}-02$ & $6.772 \mathrm{E}+00$ & $9.175 \mathrm{E}-01$ \\
\hline \multicolumn{10}{|l|}{ Formic Acid, dimer } \\
\hline \multicolumn{10}{|l|}{ Hydrofluoride, dimer } \\
\hline 2,2'-Iminobisacetic acid & & $3.775 \mathrm{E}-02$ & $1.733 \mathrm{E}+01$ & $1.733 \mathrm{E}+01$ & $1.733 \mathrm{E}+01$ & & $3.767 \mathrm{E}-02$ & $1.730 \mathrm{E}+01$ & \\
\hline Water & & $8.703 \mathrm{E}+01$ & $3.996 \mathrm{E}+04$ & $4.006 \mathrm{E}+04$ & $4.006 \mathrm{E}+04$ & & $8.705 \mathrm{E}+01$ & $3.997 \mathrm{E}+04$ & $9.343 \mathrm{E}+02$ \\
\hline \multicolumn{10}{|l|}{ Sulfuric Acid } \\
\hline Hydrogen Chloride & & $3.614 \mathrm{E}-01$ & $1.659 \mathrm{E}+02$ & $1.659 \mathrm{E}+02$ & $1.659 \mathrm{E}+02$ & & $3.606 \mathrm{E}-01$ & $1.656 \mathrm{E}+02$ & \\
\hline Formic Acid & & $1.320 \mathrm{E}-02$ & $6.063 \mathrm{E}+00$ & $6.063 \mathrm{E}+00$ & $6.063 \mathrm{E}+00$ & & $1.318 \mathrm{E}-02$ & $6.050 \mathrm{E}+00$ & \\
\hline Hydrofluoric Acid & & $7.795 \mathrm{E}-02$ & $3.579 \mathrm{E}+01$ & $3.573 \mathrm{E}+01$ & $3.573 \mathrm{E}+01$ & & $7.765 \mathrm{E}-02$ & $3.566 \mathrm{E}+01$ & \\
\hline \multicolumn{10}{|l|}{ Mercury (II) Chloride } \\
\hline Mercury (elemental) & & $1.933 E-08$ & $8.874 E-06$ & $8.874 E-06$ & $8.874 E-06$ & & $1.928 E-08$ & $8.855 E-06$ & \\
\hline Mercury (II) Oxide & $2.618 E+01$ & $5.138 E-03$ & $2.359 E+00$ & $2.853 E+01$ & $2.359 E+00$ & $2.618 E+01$ & $5.126 E-03$ & $2.354 E+00$ & \\
\hline Nitrous (III) Acid & & $4.748 \mathrm{E}+00$ & $2.180 \mathrm{E}+03$ & $2.180 \mathrm{E}+03$ & $2.180 \mathrm{E}+03$ & & $4.737 \mathrm{E}+00$ & $2.175 \mathrm{E}+03$ & \\
\hline Nitric Acid & & $2.125 \mathrm{E}+00$ & $9.759 \mathrm{E}+02$ & $9.759 \mathrm{E}+02$ & $9.759 \mathrm{E}+02$ & & $2.121 \mathrm{E}+00$ & $9.738 \mathrm{E}+02$ & $1.266 \mathrm{E}+02$ \\
\hline Hexachlorobutadiene & & $1.769 E-10$ & $8.123 E-08$ & $8.123 E-08$ & $8.123 E-08$ & & $1.765 E-10$ & $8.105 E-08$ & \\
\hline \multicolumn{10}{|l|}{ Potassium Citrate } \\
\hline \multicolumn{10}{|l|}{ Potassium Acetate } \\
\hline Potassium Chloride & & $1.201 \mathrm{E}+00$ & $5.515 \mathrm{E}+02$ & $5.515 \mathrm{E}+02$ & $5.515 \mathrm{E}+02$ & & $1.198 \mathrm{E}+00$ & $5.503 \mathrm{E}+02$ & \\
\hline
\end{tabular}


WSRC-TR-2002-00550, Rev. 1 SRT-RPP-2002-0027.

\begin{tabular}{|c|c|c|c|c|c|c|c|c|c|}
\hline Stream & $\begin{array}{l}\text { SCW4 } \\
\text { Solids }\end{array}$ & $\begin{array}{l}\text { SCW4 } \\
\text { Recycle }\end{array}$ & $\begin{array}{l}\text { SCW4 } \\
\text { Return }\end{array}$ & $\begin{array}{l}\text { SCWash5 } \\
\text { Slurry }\end{array}$ & $\begin{array}{l}\text { SCW5 } \\
\text { Liquor }\end{array}$ & $\begin{array}{l}\text { SCW5 } \\
\text { Solids }\end{array}$ & $\begin{array}{l}\text { SCW5 } \\
\text { Recycle }\end{array}$ & $\begin{array}{l}\text { SCW5 } \\
\text { Return }\end{array}$ & Acid Rinse \\
\hline Potassium Glycolate & & $1.730 \mathrm{E}-01$ & $7.942 \mathrm{E}+01$ & $7.942 \mathrm{E}+01$ & $7.942 \mathrm{E}+01$ & & $1.726 \mathrm{E}-01$ & $7.925 \mathrm{E}+01$ & \\
\hline \multicolumn{10}{|l|}{ Potassium Nitrate (VI) } \\
\hline gamma-BHC (Lindane) & & $4.926 E-07$ & $2.262 E-04$ & $2.262 E-04$ & $2.262 E-04$ & & $4.915 E-07$ & $2.257 E-04$ & \\
\hline $\begin{array}{c}\text { 4-methyl-2-pentanone } \\
(\text { MIBK) }\end{array}$ & & $9.406 E-08$ & $4.319 E-05$ & $4.319 E-05$ & $4.319 E-05$ & & $9.385 E-08$ & $4.310 E-05$ & \\
\hline \multicolumn{10}{|l|}{ Magnesium Oxalate } \\
\hline Magnesium Dichromate (VI) & & $5.422 \mathrm{E}-09$ & $2.490 \mathrm{E}-06$ & $2.504 \mathrm{E}-06$ & $2.504 \mathrm{E}-06$ & & 5.442E-09 & $2.499 \mathrm{E}-06$ & \\
\hline Magnesium Hydroxide & $2.385 \mathrm{E}+02$ & & & $2.385 \mathrm{E}+02$ & & $2.385 \mathrm{E}+02$ & & & $4.297 \mathrm{E}-02$ \\
\hline $\begin{array}{l}\text { Manganese (II) } \\
\text { Orthophosphate }\end{array}$ & & $3.613 \mathrm{E}-05$ & $1.659 \mathrm{E}-02$ & $1.659 \mathrm{E}-02$ & $1.659 \mathrm{E}-02$ & & $3.605 \mathrm{E}-05$ & $1.655 \mathrm{E}-02$ & \\
\hline \multicolumn{10}{|l|}{ Manganese (II) Oxalate } \\
\hline Manganese (II) Hydroxide & & & & & & & & & $5.433 \mathrm{E}-02$ \\
\hline Nitrogen & & $1.940 \mathrm{E}-08$ & $8.906 \mathrm{E}-06$ & $8.906 \mathrm{E}-06$ & $8.906 \mathrm{E}-06$ & & $1.935 \mathrm{E}-08$ & $8.886 \mathrm{E}-06$ & \\
\hline Sodium Oxalate & $8.296 \mathrm{E}+03$ & & & $8.296 \mathrm{E}+03$ & & $8.296 \mathrm{E}+03$ & & & \\
\hline Sodium Chromate (VI) & & $2.212 \mathrm{E}-01$ & $1.016 \mathrm{E}+02$ & $1.016 \mathrm{E}+02$ & $1.016 \mathrm{E}+02$ & & $2.207 \mathrm{E}-01$ & $1.013 \mathrm{E}+02$ & \\
\hline Sodium Fluoride Sulfate & $8.080 \mathrm{E}+02$ & & & $7.950 \mathrm{E}+02$ & & $7.950 \mathrm{E}+02$ & & & \\
\hline \multicolumn{10}{|l|}{ Sodium Acetate } \\
\hline \multicolumn{10}{|l|}{ Sodium Glycolate } \\
\hline \multicolumn{10}{|l|}{ Sodium Nitrite } \\
\hline Sodium Hydroxide & & $2.394 \mathrm{E}+01$ & $1.099 \mathrm{E}+04$ & $1.100 \mathrm{E}+04$ & $1.100 \mathrm{E}+04$ & & $2.390 \mathrm{E}+01$ & $1.097 \mathrm{E}+04$ & $4.629 \mathrm{E}-01$ \\
\hline Naphthalene & & $2.494 \mathrm{E}-08$ & $1.145 \mathrm{E}-05$ & $1.145 \mathrm{E}-05$ & $1.145 \mathrm{E}-05$ & & $2.489 \mathrm{E}-08$ & $1.143 \mathrm{E}-05$ & \\
\hline Sodium Aluminosilicate Gel & $3.403 \mathrm{E}+03$ & & & $3.403 \mathrm{E}+03$ & & $3.403 \mathrm{E}+03$ & & & \\
\hline Nickel Orthophosphate & & $1.095 \mathrm{E}-04$ & $5.029 \mathrm{E}-02$ & $5.029 \mathrm{E}-02$ & $5.029 \mathrm{E}-02$ & & $1.093 \mathrm{E}-04$ & $5.018 \mathrm{E}-02$ & \\
\hline \multicolumn{10}{|l|}{ Nickel (II) Oxalate } \\
\hline Nickel (II) Hydroxide & & & & & & & & & $1.663 \mathrm{E}-01$ \\
\hline Oxygen & & $1.079 \mathrm{E}-08$ & $4.955 \mathrm{E}-06$ & $4.955 \mathrm{E}-06$ & $4.955 \mathrm{E}-06$ & & $1.077 \mathrm{E}-08$ & $4.944 \mathrm{E}-06$ & \\
\hline Phosphorus Pentoxide & & $2.125 \mathrm{E}-02$ & $9.758 \mathrm{E}+00$ & $9.758 \mathrm{E}+00$ & $9.758 \mathrm{E}+00$ & & $2.121 \mathrm{E}-02$ & $9.737 \mathrm{E}+00$ & \\
\hline PYRENE & & $7.746 \mathrm{E}-07$ & $3.557 \mathrm{E}-04$ & $3.557 \mathrm{E}-04$ & $3.557 \mathrm{E}-04$ & & $7.729 \mathrm{E}-07$ & $3.549 \mathrm{E}-04$ & \\
\hline Silicon Dioxide & & $1.631 \mathrm{E}-01$ & $7.490 \mathrm{E}+01$ & $7.473 \mathrm{E}+01$ & $7.473 \mathrm{E}+01$ & & $1.624 \mathrm{E}-01$ & $7.456 \mathrm{E}+01$ & $1.093 \mathrm{E}-01$ \\
\hline Sulfur Trioxide & & $1.055 \mathrm{E}+00$ & $4.842 \mathrm{E}+02$ & $4.899 \mathrm{E}+02$ & $4.899 \mathrm{E}+02$ & & $1.064 \mathrm{E}+00$ & $4.888 \mathrm{E}+02$ & \\
\hline 1,2,4-trichlorobenzene & & $5.267 \mathrm{E}-09$ & $2.418 \mathrm{E}-06$ & $2.418 \mathrm{E}-06$ & $2.418 \mathrm{E}-06$ & & $5.255 \mathrm{E}-09$ & $2.413 \mathrm{E}-06$ & \\
\hline 1,2,3-trichloropropane & & $3.712 \mathrm{E}-08$ & $1.705 \mathrm{E}-05$ & $1.705 \mathrm{E}-05$ & $1.705 \mathrm{E}-05$ & & $3.704 \mathrm{E}-08$ & $1.701 \mathrm{E}-05$ & \\
\hline TOLUENE & & $3.271 \mathrm{E}-09$ & $1.502 \mathrm{E}-06$ & $1.502 \mathrm{E}-06$ & $1.502 \mathrm{E}-06$ & & $3.263 \mathrm{E}-09$ & $1.499 \mathrm{E}-06$ & \\
\hline Zinc Dichloride Pentoxide & & $2.242 \mathrm{E}-02$ & $1.029 \mathrm{E}+01$ & $1.029 \mathrm{E}+01$ & $1.029 \mathrm{E}+01$ & & $2.237 \mathrm{E}-02$ & $1.027 \mathrm{E}+01$ & \\
\hline \multicolumn{10}{|l|}{ Zinc Oxalate } \\
\hline \multicolumn{10}{|l|}{ Zinc Hydroxide } \\
\hline \multicolumn{10}{|l|}{ Zirconium EDTA } \\
\hline Zirconium Oxide & & $1.640 \mathrm{E}-04$ & $7.532 \mathrm{E}-02$ & $7.532 \mathrm{E}-02$ & $7.532 \mathrm{E}-02$ & & $1.637 \mathrm{E}-04$ & $7.515 \mathrm{E}-02$ & $3.279 \mathrm{E}-01$ \\
\hline Total g/hr & $1.433 \mathrm{E}+04$ & $1.251 \mathrm{E}+02$ & $5.745 \mathrm{E}+04$ & $7.188 \mathrm{E}+04$ & $5.756 \mathrm{E}+04$ & $1.432 \mathrm{E}+04$ & $1.251 \mathrm{E}+02$ & $5.744 \mathrm{E}+04$ & $1.064 \mathrm{E}+03$ \\
\hline Volume, L/hr & $3.198 \mathrm{E}+00$ & $1.000 \mathrm{E}-01$ & $4.592 \mathrm{E}+01$ & $4.922 \mathrm{E}+01$ & $4.602 \mathrm{E}+01$ & $3.198 \mathrm{E}+00$ & $1.000 \mathrm{E}-01$ & $4.592 \mathrm{E}+01$ & $1.000 \mathrm{E}+00$ \\
\hline Enthalpy, cal/hr & $-4.078 \mathrm{E}+07$ & $-4.205 \mathrm{E}+05$ & $-1.931 \mathrm{E}+08$ & $-2.342 \mathrm{E}+08$ & $-1.935 \mathrm{E}+08$ & $-4.075 \mathrm{E}+07$ & $-4.205 \mathrm{E}+05$ & $-1.931 \mathrm{E}+08$ & $-3.648 \mathrm{E}+06$ \\
\hline \multicolumn{10}{|l|}{ Vapor fraction } \\
\hline Solid fraction & $1.000 \mathrm{E}+00$ & & & $3.442 \mathrm{E}-02$ & & $1.000 \mathrm{E}+00$ & & & \\
\hline \multicolumn{10}{|l|}{ Organic fraction } \\
\hline Osmotic Pres, atm & & $3.672 \mathrm{E}+02$ & $3.672 \mathrm{E}+02$ & $3.664 \mathrm{E}+02$ & $3.664 \mathrm{E}+02$ & & $3.664 \mathrm{E}+02$ & $3.664 \mathrm{E}+02$ & $9.744 \mathrm{E}+01$ \\
\hline \multicolumn{10}{|l|}{ Redox Pot, volts } \\
\hline E-Con, 1/ohm-cm & & $2.591 \mathrm{E}-01$ & $2.591 \mathrm{E}-01$ & $2.591 \mathrm{E}-01$ & $2.591 \mathrm{E}-01$ & & $2.591 \mathrm{E}-01$ & $2.591 \mathrm{E}-01$ & $5.395 \mathrm{E}-01$ \\
\hline E-Con, cm2/ohm-mol & & $2.454 \mathrm{E}+01$ & $2.454 \mathrm{E}+01$ & $2.457 \mathrm{E}+01$ & $2.457 \mathrm{E}+01$ & & $2.457 \mathrm{E}+01$ & $2.457 \mathrm{E}+01$ & $2.691 \mathrm{E}+02$ \\
\hline Abs Visc, cP & & $3.675 \mathrm{E}+00$ & $3.675 \mathrm{E}+00$ & $3.667 \mathrm{E}+00$ & $3.667 \mathrm{E}+00$ & & $3.667 \mathrm{E}+00$ & $3.667 \mathrm{E}+00$ & $9.672 \mathrm{E}-01$ \\
\hline Rel Visc & & $4.126 \mathrm{E}+00$ & $4.126 \mathrm{E}+00$ & $4.116 \mathrm{E}+00$ & $4.116 \mathrm{E}+00$ & & $4.116 \mathrm{E}+00$ & $4.116 \mathrm{E}+00$ & $1.086 \mathrm{E}+00$ \\
\hline Ionic Strength & & $6.982 \mathrm{E}+00$ & $6.982 \mathrm{E}+00$ & $6.970 \mathrm{E}+00$ & $6.970 \mathrm{E}+00$ & & $6.970 \mathrm{E}+00$ & $6.970 \mathrm{E}+00$ & $2.114 \mathrm{E}+00$ \\
\hline
\end{tabular}


WSRC-TR-2002-00550, Rev. 1

SRT-RPP-2002-0027.

Table B- 1. Waste Feed Evaporator 5 Stage Model Results for UF 1.22 Density Case (cont'd) ${ }^{8}$

\begin{tabular}{|c|c|c|c|c|c|c|c|}
\hline Stream & Total Acid & Caustic Rinse & Total C Rinse & Acc1 Recycle & Total Recycle & $\begin{array}{l}\text { HLW OG } \\
\text { Cond }\end{array}$ & Evap Feed \\
\hline Phase & Aqueous & Aqueous & Aqueous & Mixed & Mixed & Mixed & Mixed \\
\hline Temperature, $\mathrm{C}$ & 25 & 25 & 25 & 25 & 25 & 25 & 25 \\
\hline Pressure, atm & 1 & 1 & 1 & 1 & 1 & 1 & 1 \\
\hline $\mathrm{pH}$ & -0.301785 & 12.8948 & 12.8948 & 14.2654 & 13.4748 & 11.2694 & 13.0705 \\
\hline Total mol/hr & 808.918 & 55.498 & 2498.34 & 38.9656 & 4402.02 & 188132 & 197463 \\
\hline Flow Units & $\mathrm{g} / \mathrm{hr}$ & $\mathrm{g} / \mathrm{hr}$ & $\mathrm{g} / \mathrm{hr}$ & $\mathrm{g} / \mathrm{hr}$ & $\mathrm{g} / \mathrm{hr}$ & $\mathrm{g} / \mathrm{hr}$ & $\mathrm{g} / \mathrm{hr}$ \\
\hline \multicolumn{8}{|l|}{ Acetic Acid, Dimer } \\
\hline Acetic Acid & & & & $5.773 \mathrm{E}-02$ & $1.286 \mathrm{E}+00$ & & \\
\hline Aluminum Oxide & $1.822 \mathrm{E}+01$ & & & $3.044 \mathrm{E}+00$ & $1.567 \mathrm{E}+02$ & $1.231 \mathrm{E}+00$ & $2.657 \mathrm{E}+03$ \\
\hline Aldrin & & & & $7.043 E-06$ & $1.568 E-04$ & & $4.195 E+00$ \\
\hline Aluminum Hydrogen EDTA & & & & & & & $1.741 \mathrm{E}+03$ \\
\hline Aluminum Hydroxide & & & & & $6.264 \mathrm{E}+01$ & & $3.103 \mathrm{E}+03$ \\
\hline Benzene & & & & $3.475 E-08$ & $7.739 E-07$ & & $4.194 E+00$ \\
\hline $\begin{array}{c}\text { Bis(2-ethylhexyl)phthalate } \\
\text { (BEHP) }\end{array}$ & & & & $8.851 E-05$ & $1.971 E-03$ & & $4.193 E+00$ \\
\hline Benzo(a)pyrene (BaP) & & & & $5.431 E-07$ & $9.236 E-06$ & & $4.181 E+00$ \\
\hline Phenol & & & & $1.234 E-03$ & $2.747 E-02$ & & $4.196 E+00$ \\
\hline Calcium Chloride Oxide & & & & & & $5.464 \mathrm{E}+02$ & \\
\hline Calcium Oxalate & & & & & & $5.512 \mathrm{E}+00$ & $6.736 \mathrm{E}+02$ \\
\hline Calcium Oxalate Monohydrate & & & & & & $2.059 \mathrm{E}+00$ & \\
\hline Calcium Carbonate & & & & & $2.337 \mathrm{E}+00$ & & \\
\hline Calcium Dichromate (VI) & & & & $2.768 \mathrm{E}-01$ & $2.770 \mathrm{E}+00$ & & \\
\hline Calcium Fluoride & & & & & & $5.358 \mathrm{E}+02$ & $3.237 \mathrm{E}+02$ \\
\hline \multicolumn{8}{|l|}{ Calcium Hydroxide } \\
\hline Pentachlorophenol & & & & $1.065 E-04$ & $2.372 E-03$ & & $4.196 E+00$ \\
\hline Hexachlorobenzene & & & & $1.296 E-06$ & $2.205 E-05$ & & $4.188 E+00$ \\
\hline Chlorobenzene & & & & $7.453 E-08$ & $1.660 E-06$ & & $4.190 E+00$ \\
\hline 2-Chloronaphthalene & & & & $2.002 E-07$ & $4.459 E-06$ & & $4.194 E+00$ \\
\hline Carbon Dioxide & & & & $2.311 \mathrm{E}+01$ & $5.136 \mathrm{E}+02$ & & $7.847 \mathrm{E}+04$ \\
\hline Cesium Acetate & & & & & & & $1.972 \mathrm{E}+02$ \\
\hline Cesium Glycolate & & & & $6.292 \mathrm{E}-02$ & $1.401 \mathrm{E}+00$ & & \\
\hline Dibenz[a,h]anthracene & & & & $4.948 E-05$ & $1.102 E-03$ & & $4.191 E+00$ \\
\hline 1,2-dibromoethane & & & & $1.791 E-06$ & $3.989 E-05$ & & $2.806 E+01$ \\
\hline Diethyl phthalate & & & & $2.691 E-04$ & $5.992 E-03$ & & $4.202 E+00$ \\
\hline Iron (III) Oxalate & & & & $2.528 \mathrm{E}-02$ & $3.730 \mathrm{E}-02$ & $1.343 \mathrm{E}+00$ & \\
\hline \multicolumn{8}{|l|}{ Iron (III) Citrate } \\
\hline Iron (III) Hydrogen EDTA & & & & $5.554 \mathrm{E}-01$ & $1.097 \mathrm{E}+01$ & & \\
\hline Iron (III) Hydroxide & $1.377 \mathrm{E}+01$ & & & $3.613 \mathrm{E}-02$ & $2.919 \mathrm{E}+01$ & $1.547 \mathrm{E}+02$ & \\
\hline \multicolumn{8}{|l|}{ Formic Acid, dimer } \\
\hline \multicolumn{8}{|l|}{ Hydrofluoride, dimer } \\
\hline 2,2'-Iminobisacetic acid & & & & $3.334 \mathrm{E}-01$ & $7.425 \mathrm{E}+00$ & & $1.132 \mathrm{E}+03$ \\
\hline Water & $1.402 \mathrm{E}+04$ & $9.980 \mathrm{E}+02$ & $4.493 \mathrm{E}+04$ & $5.997 \mathrm{E}+02$ & $7.570 \mathrm{E}+04$ & $3.361 \mathrm{E}+06$ & $3.290 \mathrm{E}+06$ \\
\hline \multicolumn{8}{|l|}{ Sulfuric Acid } \\
\hline Hydrogen Chloride & & & & $3.515 \mathrm{E}+00$ & $7.828 \mathrm{E}+01$ & $1.862 \mathrm{E}+04$ & \\
\hline Formic Acid & & & & $1.166 \mathrm{E}-01$ & $2.597 \mathrm{E}+00$ & & $3.961 \mathrm{E}+02$ \\
\hline Hydrofluoric Acid & & & & $8.527 \mathrm{E}-01$ & $1.559 \mathrm{E}+01$ & $2.116 \mathrm{E}+01$ & $3.388 \mathrm{E}+03$ \\
\hline \multicolumn{8}{|l|}{ Mercury (II) Chloride } \\
\hline Mercury (elemental) & & & & $1.707 E-07$ & $3.801 E-06$ & & \\
\hline Mercury (II) Oxide & & & & $1.943 E-02$ & $9.851 E-01$ & & $1.361 E+02$ \\
\hline Nitrous (III) Acid & & & & $4.193 \mathrm{E}+01$ & $9.338 \mathrm{E}+02$ & $3.708 \mathrm{E}+03$ & $9.342 \mathrm{E}+04$ \\
\hline Nitric Acid & $1.899 \mathrm{E}+03$ & & & $1.877 \mathrm{E}+01$ & $2.317 \mathrm{E}+03$ & $1.888 \mathrm{E}+03$ & $4.525 \mathrm{E}+04$ \\
\hline Hexachlorobutadiene & & & & $1.562 E-09$ & $3.479 E-08$ & & $4.200 E+00$ \\
\hline Potassium Citrate & & & & $8.313 \mathrm{E}-01$ & $1.851 \mathrm{E}+01$ & & $2.823 \mathrm{E}+03$ \\
\hline Potassium Acetate & & & & & & & $2.195 \mathrm{E}+02$ \\
\hline Potassium Chloride & & & & $1.000 \mathrm{E}+01$ & $2.227 \mathrm{E}+02$ & $1.845 \mathrm{E}+04$ & $1.350 \mathrm{E}+03$ \\
\hline
\end{tabular}


WSRC-TR-2002-00550, Rev. 1 SRT-RPP-2002-0027.

\begin{tabular}{|c|c|c|c|c|c|c|c|}
\hline Stream & Total Acid & Caustic Rinse & Total C Rinse & Acc1 Recycle & Total Recycle & $\begin{array}{l}\text { HLW OG } \\
\text { Cond }\end{array}$ & Evap Feed \\
\hline Potassium Glycolate & & & & $1.528 \mathrm{E}+00$ & $3.402 \mathrm{E}+01$ & & \\
\hline Potassium Nitrate (VI) & & & & & & & $2.357 \mathrm{E}+04$ \\
\hline gamma-BHC (Lindane) & & & & $4.351 E-06$ & $9.689 E-05$ & & $4.209 E+00$ \\
\hline $\begin{array}{l}\text { 4-methyl-2-pentanone } \\
\text { (MIBK) }\end{array}$ & & & & $8.308 E-07$ & $1.850 E-05$ & & $4.197 E+00$ \\
\hline Magnesium Oxalate & & & & & & $2.060 \mathrm{E}+00$ & \\
\hline Magnesium Dichromate (VI) & & & & $9.765 \mathrm{E}-07$ & $1.612 \mathrm{E}-04$ & & \\
\hline Magnesium Hydroxide & $6.449 \mathrm{E}-01$ & & & $1.653 \mathrm{E}-06$ & $6.448 \mathrm{E}-01$ & $2.368 \mathrm{E}+02$ & \\
\hline \multicolumn{8}{|l|}{$\begin{array}{l}\text { Manganese (II) } \\
\text { Orthophosphate }\end{array}$} \\
\hline Manganese (II) Oxalate & & & & $3.858 \mathrm{E}-04$ & $1.182 \mathrm{E}-01$ & & \\
\hline Manganese (II) Hydroxide & $8.152 \mathrm{E}-01$ & & & & $7.470 \mathrm{E}-01$ & & \\
\hline Nitrogen & & & & $1.713 \mathrm{E}-07$ & $3.815 \mathrm{E}-06$ & & \\
\hline Sodium Oxalate & & & & $1.364 \mathrm{E}+00$ & $2.103 \mathrm{E}+00$ & & $1.433 \mathrm{E}+04$ \\
\hline Sodium Chromate (VI) & & & & $2.108 \mathrm{E}+00$ & $5.125 \mathrm{E}+01$ & & $8.349 \mathrm{E}+03$ \\
\hline \multicolumn{8}{|l|}{ Sodium Fluoride Sulfate } \\
\hline \multicolumn{8}{|l|}{ Sodium Acetate } \\
\hline Sodium Glycolate & & & & & & & $4.556 \mathrm{E}+03$ \\
\hline Sodium Nitrite & & & & & & $3.054 \mathrm{E}+03$ & $6.342 \mathrm{E}+04$ \\
\hline Sodium Hydroxide & $6.946 \mathrm{E}+00$ & $4.000 \mathrm{E}+00$ & $1.801 \mathrm{E}+02$ & $1.376 \mathrm{E}+02$ & $4.790 \mathrm{E}+03$ & $2.572 \mathrm{E}+04$ & $3.253 \mathrm{E}+05$ \\
\hline Naphthalene & & & & $2.203 \mathrm{E}-07$ & $4.906 \mathrm{E}-06$ & & $4.188 \mathrm{E}+00$ \\
\hline Sodium Aluminosilicate Gel & & & & $2.971 \mathrm{E}-01$ & $8.103 \mathrm{E}+01$ & $2.920 \mathrm{E}+03$ & \\
\hline \multicolumn{8}{|l|}{ Nickel Orthophosphate } \\
\hline Nickel (II) Oxalate & & & & $1.163 \mathrm{E}-03$ & $3.977 \mathrm{E}+00$ & & \\
\hline Nickel (II) Hydroxide & $2.496 \mathrm{E}+00$ & & & & & & \\
\hline Oxygen & & & & $9.531 \mathrm{E}-08$ & $2.122 \mathrm{E}-06$ & & \\
\hline Phosphorus Pentoxide & & & & $1.882 \mathrm{E}-01$ & $4.191 \mathrm{E}+00$ & & $6.391 \mathrm{E}+02$ \\
\hline PYRENE & & & & $6.842 \mathrm{E}-06$ & $1.524 \mathrm{E}-04$ & & $4.202 \mathrm{E}+00$ \\
\hline Silicon Dioxide & $1.640 \mathrm{E}+00$ & & & $2.422 \mathrm{E}-01$ & $3.561 \mathrm{E}+00$ & $3.339 \mathrm{E}+02$ & \\
\hline Sulfur Trioxide & & & & $1.286 \mathrm{E}+01$ & $2.021 \mathrm{E}+02$ & $5.781 \mathrm{E}+02$ & $4.702 \mathrm{E}+04$ \\
\hline 1,2,4-trichlorobenzene & & & & $4.652 \mathrm{E}-08$ & $1.036 \mathrm{E}-06$ & & $4.193 \mathrm{E}+00$ \\
\hline 1,2,3-trichloropropane & & & & $3.279 \mathrm{E}-07$ & $7.301 \mathrm{E}-06$ & & $4.198 \mathrm{E}+00$ \\
\hline TOLUENE & & & & $2.889 \mathrm{E}-08$ & $6.433 \mathrm{E}-07$ & & $4.194 \mathrm{E}+00$ \\
\hline Zinc Dichloride Pentoxide & & & & & & $9.198 \mathrm{E}+00$ & \\
\hline Zinc Oxalate & & & & 3.355E-01 & $7.471 \mathrm{E}+00$ & & \\
\hline Zinc Hydroxide & & & & & & $7.281 \mathrm{E}+02$ & \\
\hline Zirconium EDTA & & & & $4.461 \mathrm{E}-03$ & $1.634 \mathrm{E}+00$ & & \\
\hline Zirconium Oxide & $4.920 \mathrm{E}+00$ & & & & $4.421 \mathrm{E}+00$ & & \\
\hline Total g/hr & $1.597 \mathrm{E}+04$ & $1.002 \mathrm{E}+03$ & $4.511 \mathrm{E}+04$ & $8.598 \mathrm{E}+02$ & $8.526 \mathrm{E}+04$ & $3.438 \mathrm{E}+06$ & $4.012 \mathrm{E}+06$ \\
\hline Volume, L/hr & $1.501 \mathrm{E}+01$ & $1.001 \mathrm{E}+00$ & $4.505 \mathrm{E}+01$ & 6.999E-01 & $7.946 \mathrm{E}+01$ & $3.405 \mathrm{E}+03$ & $3.551 \mathrm{E}+03$ \\
\hline Enthalpy, cal/hr & $-5.475 \mathrm{E}+07$ & $-3.796 \mathrm{E}+06$ & $-1.709 \mathrm{E}+08$ & $-2.855 \mathrm{E}+06$ & $-3.074 \mathrm{E}+08$ & $-1.289 \mathrm{E}+10$ & $-1.404 \mathrm{E}+10$ \\
\hline \multicolumn{8}{|l|}{ Vapor fraction } \\
\hline Solid fraction & & & & $7.960 \mathrm{E}-05$ & $2.782 \mathrm{E}-04$ & $1.181 \mathrm{E}-04$ & $2.238 \mathrm{E}-04$ \\
\hline \multicolumn{8}{|l|}{ Organic fraction } \\
\hline Osmotic Pres, atm & $9.744 \mathrm{E}+01$ & $4.622 \mathrm{E}+00$ & $4.622 \mathrm{E}+00$ & $2.223 \mathrm{E}+02$ & $6.077 \mathrm{E}+01$ & $1.256 \mathrm{E}+01$ & $9.071 \mathrm{E}+01$ \\
\hline \multicolumn{8}{|l|}{ Redox Pot, volts } \\
\hline E-Con, 1/ohm-cm & $5.395 \mathrm{E}-01$ & $2.233 \mathrm{E}-02$ & $2.233 \mathrm{E}-02$ & $2.249 \mathrm{E}-01$ & $1.235 \mathrm{E}-01$ & $2.639 \mathrm{E}-02$ & $1.378 \mathrm{E}-01$ \\
\hline E-Con, cm2/ohm-mol & $2.691 \mathrm{E}+02$ & $2.235 \mathrm{E}+02$ & $2.235 \mathrm{E}+02$ & $2.899 \mathrm{E}+01$ & $4.880 \mathrm{E}+01$ & $9.004 \mathrm{E}+01$ & $6.830 \mathrm{E}+01$ \\
\hline Abs Visc, cP & $9.672 \mathrm{E}-01$ & $9.101 \mathrm{E}-01$ & $9.101 \mathrm{E}-01$ & $2.637 \mathrm{E}+00$ & $1.150 \mathrm{E}+00$ & $9.062 \mathrm{E}-01$ & $1.494 \mathrm{E}+00$ \\
\hline Rel Visc & $1.086 \mathrm{E}+00$ & $1.022 \mathrm{E}+00$ & $1.022 \mathrm{E}+00$ & $2.961 \mathrm{E}+00$ & $1.291 \mathrm{E}+00$ & $1.017 \mathrm{E}+00$ & $1.678 \mathrm{E}+00$ \\
\hline Ionic Strength & $2.114 \mathrm{E}+00$ & $1.002 \mathrm{E}-01$ & $1.002 \mathrm{E}-01$ & $5.949 \mathrm{E}+00$ & $1.596 \mathrm{E}+00$ & $2.815 \mathrm{E}-01$ & $3.062 \mathrm{E}+00$ \\
\hline
\end{tabular}


Table B - 2. Waste Feed Evaporator 5 Stage Model Results for UF 1.35 Density Case ${ }^{8}$

\begin{tabular}{|c|c|c|c|c|c|c|c|c|c|}
\hline Stream & $\begin{array}{c}\text { Air } \\
\text { Inleakage }\end{array}$ & $\begin{array}{c}\text { EVAP } \\
\text { REDRXN } \\
\text { OUT }\end{array}$ & $\begin{array}{c}\text { Evap } \\
\text { Contents }\end{array}$ & Overhead & $\begin{array}{c}\text { Evap } \\
\text { Bottoms }\end{array}$ & \begin{tabular}{|c|} 
Cooled Evap \\
Bott
\end{tabular} & UF1 Filtrate & UF1 Slurry & CWash-In \\
\hline Phase & Vapor & Mixed & Mixed & Vapor & Mixed & Mixed & Aqueous & Mixed & Aqueous \\
\hline Temperature, $\mathrm{C}$ & 25 & 50 & 50 & 50 & 50 & 25 & 25 & 25 & 25 \\
\hline Pressure, atm & 1 & 0.108335 & 0.102137 & 0.102137 & 0.102137 & 1 & 1 & 1 & 1 \\
\hline $\mathrm{pH}$ & & 12.6386 & 13.1314 & & 13.1314 & 14.034 & 14.034 & 14.034 & 11.9511 \\
\hline Total mol/hr & 135.18 & 393540 & 393679 & 321648 & 72031 & 71852.2 & 66180.6 & 5671.64 & 55.346 \\
\hline Flow Units & $\mathrm{g} / \mathrm{hr}$ & $\mathrm{g} / \mathrm{hr}$ & $\mathrm{g} / \mathrm{hr}$ & $\mathrm{g} / \mathrm{hr}$ & $\mathrm{g} / \mathrm{hr}$ & $\mathrm{g} / \mathrm{hr}$ & $\mathrm{g} / \mathrm{hr}$ & $\mathrm{g} / \mathrm{hr}$ & $\mathrm{g} / \mathrm{hr}$ \\
\hline Acetic Acid, Dimer & & $1.584 \mathrm{E}-18$ & $1.194 \mathrm{E}-18$ & $1.194 \mathrm{E}-18$ & & & & & \\
\hline Acetic Acid & & $2.804 \mathrm{E}-06$ & $2.715 \mathrm{E}-06$ & $2.715 \mathrm{E}-06$ & & & & & \\
\hline Aluminum Oxide & & $4.704 \mathrm{E}+03$ & $4.740 \mathrm{E}+03$ & & $4.740 \mathrm{E}+03$ & $4.737 \mathrm{E}+03$ & $4.375 \mathrm{E}+03$ & $3.615 \mathrm{E}+02$ & \\
\hline Aldrin & & $4.195 E+00$ & $4.195 E+00$ & $4.189 E+00$ & $5.701 E-03$ & $5.701 E-03$ & $5.266 E-03$ & $4.351 E-04$ & \\
\hline Aluminum Hydrogen EDTA & & $1.209 \mathrm{E}+03$ & $1.208 \mathrm{E}+03$ & & $1.208 \mathrm{E}+03$ & $1.188 \mathrm{E}+03$ & $1.097 \mathrm{E}+03$ & $9.067 \mathrm{E}+01$ & \\
\hline \multicolumn{10}{|l|}{ Aluminum Hydroxide } \\
\hline Benzene & & $4.194 E+00$ & $4.194 E+00$ & $4.194 E+00$ & $2.799 E-05$ & $2.799 E-05$ & $2.585 E-05$ & $2.136 E-06$ & \\
\hline $\begin{array}{c}\text { Bis(2-ethylhexyl)phthalate } \\
\text { (BEHP) }\end{array}$ & & $4.194 E+00$ & $4.194 E+00$ & $4.119 E+00$ & $7.548 E-02$ & $7.548 E-02$ & $6.972 E-02$ & $5.760 E-03$ & \\
\hline Benzo(a)pyrene (BaP) & & $4.181 E+00$ & $4.181 E+00$ & & $4.181 E+00$ & $4.181 E+00$ & $5.716 E-04$ & $4.181 E+00$ & \\
\hline Phenol & & $4.249 E+00$ & $4.249 E+00$ & $4.512 E-03$ & $4.245 E+00$ & $4.245 E+00$ & $3.921 E+00$ & $3.239 E-01$ & \\
\hline \multicolumn{10}{|l|}{ Calcium Chloride Oxide } \\
\hline Calcium Oxalate & & $2.141 \mathrm{E}+02$ & $3.096 \mathrm{E}+02$ & & $3.096 \mathrm{E}+02$ & $6.266 \mathrm{E}+02$ & $5.788 \mathrm{E}+02$ & $4.782 \mathrm{E}+01$ & \\
\hline \multicolumn{10}{|l|}{ Calcium Oxalate Monohydrate } \\
\hline \multicolumn{10}{|l|}{ Calcium Carbonate } \\
\hline \multicolumn{10}{|l|}{ Calcium Dichromate (VI) } \\
\hline \multicolumn{10}{|l|}{ Calcium Citrate } \\
\hline Calcium Fluoride & & $1.660 \mathrm{E}+03$ & $1.601 \mathrm{E}+03$ & & $1.601 \mathrm{E}+03$ & $1.408 \mathrm{E}+03$ & & $1.408 \mathrm{E}+03$ & \\
\hline \multicolumn{10}{|l|}{ Calcium Hydroxide } \\
\hline Pentachlorophenol & & $4.197 E+00$ & $4.197 E+00$ & $4.106 E+00$ & $9.188 E-02$ & $9.188 E-02$ & $8.487 E-02$ & $7.012 E-03$ & \\
\hline Hexachlorobenzene & & $4.188 E+00$ & $4.188 E+00$ & & $4.188 E+00$ & $4.188 E+00$ & $1.364 E-03$ & $4.186 E+00$ & \\
\hline Chlorobenzene & & $4.190 E+00$ & $4.190 E+00$ & $4.190 E+00$ & $6.003 E-05$ & $6.003 E-05$ & $5.545 E-05$ & $4.581 E-06$ & \\
\hline 2-Chloronaphthalene & & $4.194 E+00$ & $4.194 E+00$ & $4.194 E+00$ & $1.613 E-04$ & $1.613 E-04$ & $1.490 E-04$ & $1.231 E-05$ & \\
\hline Carbon Dioxide & $1.949 \mathrm{E}+00$ & $7.948 \mathrm{E}+04$ & $7.948 \mathrm{E}+04$ & $5.045 \mathrm{E}-02$ & $7.948 \mathrm{E}+04$ & $7.948 \mathrm{E}+04$ & $7.341 \mathrm{E}+04$ & $6.065 \mathrm{E}+03$ & \\
\hline Cesium Acetate & & $1.998 \mathrm{E}+02$ & $1.998 \mathrm{E}+02$ & & $1.998 \mathrm{E}+02$ & $1.998 \mathrm{E}+02$ & $1.845 \mathrm{E}+02$ & $1.524 \mathrm{E}+01$ & \\
\hline \multicolumn{10}{|l|}{ Cesium Glycolate } \\
\hline Dibenz[a,h]anthracene & & $4.191 E+00$ & $4.191 E+00$ & $4.150 E+00$ & $4.114 E-02$ & $4.114 E-02$ & $3.800 E-02$ & $3.140 E-03$ & \\
\hline 1,2-dibromoethane & & $2.806 E+01$ & $2.806 E+01$ & $2.806 E+01$ & $1.443 E-03$ & $1.443 E-03$ & $1.333 E-03$ & $1.101 E-04$ & \\
\hline Diethyl phthalate & & $4.205 E+00$ & $4.205 E+00$ & $3.945 E+00$ & $2.601 E-01$ & $2.601 E-01$ & $2.403 E-01$ & $1.985 E-02$ & \\
\hline \multicolumn{10}{|l|}{ Iron (III) Oxalate } \\
\hline Iron (III) Hydrogen EDTA & & $5.994 \mathrm{E}+02$ & $5.994 \mathrm{E}+02$ & & $5.994 \mathrm{E}+02$ & $5.994 \mathrm{E}+02$ & $5.537 \mathrm{E}+02$ & $4.574 \mathrm{E}+01$ & \\
\hline \multicolumn{10}{|l|}{ Iron (III) Hydroxide } \\
\hline Formic Acid, dimer & & $7.829 \mathrm{E}-25$ & $6.209 \mathrm{E}-25$ & $6.209 \mathrm{E}-25$ & & & & & \\
\hline Hydrofluoride, dimer & & $2.203 \mathrm{E}-22$ & $1.184 \mathrm{E}-22$ & $1.184 \mathrm{E}-22$ & & & & & \\
\hline 2,2'-Iminobisacetic acid & & $1.147 \mathrm{E}+03$ & $1.147 \mathrm{E}+03$ & & $1.147 \mathrm{E}+03$ & $1.147 \mathrm{E}+03$ & $1.059 \mathrm{E}+03$ & $8.752 \mathrm{E}+01$ & \\
\hline Water & $1.819 \mathrm{E}+01$ & $6.789 \mathrm{E}+06$ & $6.790 \mathrm{E}+06$ & $5.792 \mathrm{E}+06$ & $9.977 \mathrm{E}+05$ & $1.001 \mathrm{E}+06$ & $9.246 \mathrm{E}+05$ & $7.639 \mathrm{E}+04$ & $9.969 \mathrm{E}+02$ \\
\hline \multicolumn{10}{|l|}{ Sulfuric Acid } \\
\hline Hydrogen Chloride & & $1.041 \mathrm{E}+04$ & $1.041 \mathrm{E}+04$ & $4.263 \mathrm{E}-11$ & $1.041 \mathrm{E}+04$ & $1.041 \mathrm{E}+04$ & $9.617 \mathrm{E}+03$ & $7.945 \mathrm{E}+02$ & \\
\hline Formic Acid & & $4.011 \mathrm{E}+02$ & $4.011 \mathrm{E}+02$ & $3.091 \mathrm{E}-07$ & $4.011 \mathrm{E}+02$ & $4.011 \mathrm{E}+02$ & $3.705 \mathrm{E}+02$ & $3.061 \mathrm{E}+01$ & \\
\hline Hydrofluoric Acid & & $3.023 \mathrm{E}+03$ & $3.053 \mathrm{E}+03$ & $1.450 \mathrm{E}-07$ & $3.053 \mathrm{E}+03$ & $1.345 \mathrm{E}+03$ & $1.242 \mathrm{E}+03$ & $1.026 \mathrm{E}+02$ & \\
\hline Mercury (II) Chloride & & $2.442 \mathrm{E}-07$ & $1.603 \mathrm{E}-07$ & $1.603 \mathrm{E}-07$ & & & & & \\
\hline Mercury (elemental) & & $6.374 \mathrm{E}+01$ & $6.374 \mathrm{E}+01$ & $6.374 \mathrm{E}+01$ & $1.380 \mathrm{E}-04$ & $1.380 \mathrm{E}-04$ & $1.275 \mathrm{E}-04$ & $1.053 \mathrm{E}-05$ & \\
\hline Mercury (II) Oxide & & $6.878 \mathrm{E}+01$ & $6.878 \mathrm{E}+01$ & & $6.878 \mathrm{E}+01$ & $6.878 \mathrm{E}+01$ & $1.794 \mathrm{E}+01$ & $5.084 \mathrm{E}+01$ & \\
\hline Nitrous (III) Acid & & $9.653 \mathrm{E}+04$ & $9.378 \mathrm{E}+04$ & $6.469 \mathrm{E}-03$ & $9.378 \mathrm{E}+04$ & $9.330 \mathrm{E}+04$ & $8.618 \mathrm{E}+04$ & $7.120 \mathrm{E}+03$ & \\
\hline Nitric Acid & & $6.653 \mathrm{E}+04$ & $6.653 \mathrm{E}+04$ & $3.522 \mathrm{E}-12$ & $6.653 \mathrm{E}+04$ & $6.653 \mathrm{E}+04$ & $6.145 \mathrm{E}+04$ & $5.077 \mathrm{E}+03$ & \\
\hline Hexachlorobutadiene & & $4.200 E+00$ & $4.200 E+00$ & $4.200 E+00$ & $1.259 E-06$ & $1.259 E-06$ & $1.163 E-06$ & $9.605 E-08$ & \\
\hline Potassium Citrate & & $2.859 \mathrm{E}+03$ & $2.859 \mathrm{E}+03$ & & $2.859 \mathrm{E}+03$ & $2.859 \mathrm{E}+03$ & $2.641 \mathrm{E}+03$ & $2.182 \mathrm{E}+02$ & \\
\hline Potassium Acetate & & & & & & & & & \\
\hline
\end{tabular}


WSRC-TR-2002-00550, Rev. 1

SRT-RPP-2002-0027.

\begin{tabular}{|c|c|c|c|c|c|c|c|c|c|}
\hline Stream & $\begin{array}{c}\text { Air } \\
\text { Inleakage }\end{array}$ & $\begin{array}{c}\text { EVAP } \\
\text { REDRXN } \\
\text { OUT }\end{array}$ & $\begin{array}{c}\text { Evap } \\
\text { Contents }\end{array}$ & Overhead & $\begin{array}{c}\text { Evap } \\
\text { Bottoms }\end{array}$ & $\begin{array}{c}\text { Cooled Evap } \\
\text { Bott }\end{array}$ & UF1 Filtrate & UF1 Slurry & CWash-In \\
\hline Potassium Chloride & & $3.783 \mathrm{E}+04$ & $3.783 \mathrm{E}+04$ & & $3.783 \mathrm{E}+04$ & $3.783 \mathrm{E}+04$ & $3.494 \mathrm{E}+04$ & $2.887 \mathrm{E}+03$ & \\
\hline \multicolumn{10}{|l|}{ Potassium Glycolate } \\
\hline \multicolumn{10}{|l|}{ Potassium Nitrate (VI) } \\
\hline gamma-BHC (Lindane) & & $4.209 E+00$ & $4.209 E+00$ & $4.205 E+00$ & $3.515 E-03$ & $3.515 E-03$ & $3.246 E-03$ & $2.682 E-04$ & \\
\hline $\begin{array}{c}\text { 4-methyl-2-pentanone } \\
\text { (MIBK) }\end{array}$ & & $4.197 E+00$ & $4.197 E+00$ & $4.196 E+00$ & $6.694 E-04$ & $6.694 E-04$ & $6.184 E-04$ & $5.109 E-05$ & \\
\hline Magnesium Oxalate & & $2.956 \mathrm{E}-02$ & $1.704 \mathrm{E}-02$ & & $1.704 \mathrm{E}-02$ & $2.043 \mathrm{E}-03$ & $1.887 \mathrm{E}-03$ & $1.559 \mathrm{E}-04$ & \\
\hline \multicolumn{10}{|l|}{ Magnesium Dichromate (VI) } \\
\hline Magnesium Hydroxide & & $2.391 \mathrm{E}+02$ & $2.391 \mathrm{E}+02$ & & $2.391 \mathrm{E}+02$ & $2.392 \mathrm{E}+02$ & & $2.392 \mathrm{E}+02$ & \\
\hline Manganese (II) Oxalate & & $2.679 \mathrm{E}+00$ & $2.679 \mathrm{E}+00$ & & $2.679 \mathrm{E}+00$ & $2.679 \mathrm{E}+00$ & $2.474 \mathrm{E}+00$ & $2.044 \mathrm{E}-01$ & \\
\hline \multicolumn{10}{|l|}{ Manganese (II) Hydroxide } \\
\hline Nitrogen & $2.970 \mathrm{E}+03$ & $1.743 \mathrm{E}-06$ & $2.970 \mathrm{E}+03$ & $2.970 \mathrm{E}+03$ & $1.379 \mathrm{E}-04$ & $1.379 \mathrm{E}-04$ & $1.274 \mathrm{E}-04$ & $1.053 \mathrm{E}-05$ & \\
\hline Sodium Oxalate & & $1.382 \mathrm{E}+04$ & $1.372 \mathrm{E}+04$ & & $1.372 \mathrm{E}+04$ & $1.339 \mathrm{E}+04$ & $1.480 \mathrm{E}+03$ & $1.191 \mathrm{E}+04$ & \\
\hline Sodium Chromate (VI) & & $8.456 \mathrm{E}+03$ & $8.456 \mathrm{E}+03$ & & $8.456 \mathrm{E}+03$ & $8.456 \mathrm{E}+03$ & $7.811 \mathrm{E}+03$ & $6.453 \mathrm{E}+02$ & \\
\hline Sodium Fluoride Sulfate & & & & & & $1.662 \mathrm{E}+04$ & & $1.662 \mathrm{E}+04$ & \\
\hline Sodium Acetate & & $1.859 \mathrm{E}+02$ & $1.859 \mathrm{E}+02$ & & $1.859 \mathrm{E}+02$ & $1.859 \mathrm{E}+02$ & $1.717 \mathrm{E}+02$ & $1.418 \mathrm{E}+01$ & \\
\hline Sodium Glycolate & & $4.615 \mathrm{E}+03$ & $4.615 \mathrm{E}+03$ & & $4.615 \mathrm{E}+03$ & $4.615 \mathrm{E}+03$ & $4.263 \mathrm{E}+03$ & $3.522 \mathrm{E}+02$ & \\
\hline Sodium Nitrite & & $7.001 \mathrm{E}+04$ & $7.404 \mathrm{E}+04$ & & $7.404 \mathrm{E}+04$ & $7.474 \mathrm{E}+04$ & $6.903 \mathrm{E}+04$ & $5.703 \mathrm{E}+03$ & \\
\hline Sodium Hydroxide & & $3.563 \mathrm{E}+05$ & $3.540 \mathrm{E}+05$ & & $3.540 \mathrm{E}+05$ & $3.430 \mathrm{E}+05$ & $3.168 \mathrm{E}+05$ & $2.617 \mathrm{E}+04$ & 3.999E-01 \\
\hline Naphthalene & & $4.188 E+00$ & $4.188 E+00$ & $4.188 E+00$ & $1.775 E-04$ & $1.775 E-04$ & $1.639 E-04$ & $1.354 E-05$ & \\
\hline Sodium Aluminosilicate Gel & & $3.894 \mathrm{E}+03$ & $3.759 \mathrm{E}+03$ & & $3.759 \mathrm{E}+03$ & $3.784 \mathrm{E}+03$ & & $3.784 \mathrm{E}+03$ & \\
\hline Nickel (II) Oxalate & & $8.077 \mathrm{E}+00$ & $8.077 \mathrm{E}+00$ & & $8.077 \mathrm{E}+00$ & $8.077 \mathrm{E}+00$ & $7.461 \mathrm{E}+00$ & $6.164 \mathrm{E}-01$ & \\
\hline \multicolumn{10}{|l|}{ Nickel (II) Hydroxide } \\
\hline Oxygen & 8.994E+02 & $9.698 \mathrm{E}-07$ & $8.994 \mathrm{E}+02$ & $8.994 \mathrm{E}+02$ & $7.674 \mathrm{E}-05$ & 7.674E-05 & $7.088 \mathrm{E}-05$ & $5.856 \mathrm{E}-06$ & \\
\hline Phosphorus Pentoxide & & $6.473 \mathrm{E}+02$ & $6.473 \mathrm{E}+02$ & & $6.473 \mathrm{E}+02$ & $6.473 \mathrm{E}+02$ & $5.979 \mathrm{E}+02$ & $4.940 \mathrm{E}+01$ & \\
\hline PYRENE & & $4.202 E+00$ & $4.202 E+00$ & $4.196 E+00$ & $5.536 E-03$ & $5.536 E-03$ & $5.114 E-03$ & $4.225 E-04$ & \\
\hline Silicon Dioxide & & $1.102 \mathrm{E}+02$ & $1.589 \mathrm{E}+02$ & & $1.589 \mathrm{E}+02$ & $1.502 \mathrm{E}+02$ & $1.387 \mathrm{E}+02$ & $1.146 \mathrm{E}+01$ & \\
\hline Sulfur Trioxide & & $4.770 \mathrm{E}+04$ & $4.770 \mathrm{E}+04$ & & $4.770 \mathrm{E}+04$ & $4.047 \mathrm{E}+04$ & $3.738 \mathrm{E}+04$ & $3.088 \mathrm{E}+03$ & \\
\hline 1,2,4-trichlorobenzene & & $4.193 E+00$ & $4.193 E+00$ & $4.193 E+00$ & $3.747 E-05$ & $3.747 E-05$ & $3.461 E-05$ & $2.859 E-06$ & \\
\hline 1,2,3-trichloropropane & & $4.198 E+00$ & $4.198 E+00$ & $4.198 E+00$ & $2.641 E-04$ & $2.641 E-04$ & $2.440 E-04$ & $2.016 E-05$ & \\
\hline TOLUENE & & $4.194 E+00$ & $4.194 E+00$ & $4.194 E+00$ & $2.327 E-05$ & $2.327 E-05$ & $2.149 E-05$ & $1.776 E-06$ & \\
\hline \multicolumn{10}{|l|}{ Zinc Dichloride Pentoxide } \\
\hline Zinc Oxalate & & $1.154 \mathrm{E}+03$ & $1.154 \mathrm{E}+03$ & & $1.154 \mathrm{E}+03$ & $1.154 \mathrm{E}+03$ & $1.066 \mathrm{E}+03$ & $8.805 \mathrm{E}+01$ & \\
\hline \multicolumn{10}{|l|}{ Zinc Hydroxide } \\
\hline Zirconium EDTA & & $6.570 \mathrm{E}+00$ & $7.143 \mathrm{E}+00$ & & $7.143 \mathrm{E}+00$ & $3.098 \mathrm{E}+01$ & $2.861 \mathrm{E}+01$ & $2.364 \mathrm{E}+00$ & \\
\hline Zirconium Oxide & & $7.926 \mathrm{E}+00$ & $7.740 \mathrm{E}+00$ & & $7.740 \mathrm{E}+00$ & & & & \\
\hline Total g/hr & $3.889 \mathrm{E}+03$ & $7.603 \mathrm{E}+06$ & $7.607 \mathrm{E}+06$ & $5.796 \mathrm{E}+06$ & $1.811 \mathrm{E}+06$ & $1.811 \mathrm{E}+06$ & $1.641 \mathrm{E}+06$ & $1.695 \mathrm{E}+05$ & $9.973 \mathrm{E}+02$ \\
\hline Volume, L/hr & $3.307 \mathrm{E}+03$ & $6.703 \mathrm{E}+07$ & $8.341 \mathrm{E}+07$ & $8.341 \mathrm{E}+07$ & $1.368 \mathrm{E}+03$ & $1.319 \mathrm{E}+03$ & $1.216 \mathrm{E}+03$ & $1.039 \mathrm{E}+02$ & $1.000 \mathrm{E}+00$ \\
\hline Enthalpy, cal/hr & $-6.275 \mathrm{E}+04$ & $-2.450 \mathrm{E}+10$ & $-2.402 \mathrm{E}+10$ & $-1.852 \mathrm{E}+10$ & $-5.498 \mathrm{E}+09$ & $-5.522 \mathrm{E}+09$ & $-5.017 \mathrm{E}+09$ & $-5.049 \mathrm{E}+08$ & $-3.781 \mathrm{E}+06$ \\
\hline Vapor fraction & $1.000 \mathrm{E}+00$ & $6.967 \mathrm{E}-01$ & $8.170 \mathrm{E}-01$ & $1.000 \mathrm{E}+00$ & & & & & \\
\hline Solid fraction & & $1.349 \mathrm{E}-04$ & $2.539 \mathrm{E}-04$ & & $1.387 \mathrm{E}-03$ & $2.838 \mathrm{E}-03$ & & $3.596 \mathrm{E}-02$ & \\
\hline \multicolumn{10}{|l|}{ Organic fraction } \\
\hline Osmotic Pres, atm & & $1.801 \mathrm{E}+02$ & $2.724 \mathrm{E}+02$ & & $2.724 \mathrm{E}+02$ & $2.716 \mathrm{E}+02$ & $2.716 \mathrm{E}+02$ & $2.716 \mathrm{E}+02$ & $4.741 \mathrm{E}-01$ \\
\hline \multicolumn{10}{|l|}{ Redox Pot, volts } \\
\hline E-Con, 1/ohm-cm & & $2.765 \mathrm{E}-01$ & $3.799 \mathrm{E}-01$ & & $3.799 \mathrm{E}-01$ & $2.596 \mathrm{E}-01$ & & & $2.366 \mathrm{E}-03$ \\
\hline E-Con, cm2/ohm-mol & & $3.659 \mathrm{E}+01$ & $3.077 \mathrm{E}+01$ & & $3.077 \mathrm{E}+01$ & $2.052 \mathrm{E}+01$ & & & $2.367 \mathrm{E}+02$ \\
\hline Abs Visc, cP & & $1.449 \mathrm{E}+00$ & $2.382 \mathrm{E}+00$ & & $2.382 \mathrm{E}+00$ & $4.188 \mathrm{E}+00$ & & & $8.929 \mathrm{E}-01$ \\
\hline Rel Visc & & $2.648 \mathrm{E}+00$ & $4.353 \mathrm{E}+00$ & & $4.353 \mathrm{E}+00$ & $4.701 \mathrm{E}+00$ & & & $1.002 \mathrm{E}+00$ \\
\hline Ionic Strength & & $5.935 \mathrm{E}+00$ & $1.052 \mathrm{E}+01$ & & $1.052 \mathrm{E}+01$ & $9.970 \mathrm{E}+00$ & $9.970 \mathrm{E}+00$ & $9.970 \mathrm{E}+00$ & $1.003 \mathrm{E}-02$ \\
\hline
\end{tabular}


WSRC-TR-2002-00550, Rev. 1

SRT-RPP-2002-0027.

Table B- 2. Waste Feed Evaporator 5 Stage Model Results for UF 1.35 Density Case (cont'd) ${ }^{8}$

\begin{tabular}{|c|c|c|c|c|c|c|c|c|c|}
\hline Stream & $\begin{array}{l}\text { Total } \\
\text { CWash }\end{array}$ & $\begin{array}{c}\text { FCWash } \\
\text { Total }\end{array}$ & $\begin{array}{l}\text { SCWash } \\
\text { Total }\end{array}$ & FCWash-1 & FCWash-2 & FCWash-3 & FCWash-4 & FCWash-5 & SCWash-1 \\
\hline Phase & Aqueous & Aqueous & Aqueous & Aqueous & Aqueous & Aqueous & Aqueous & Aqueous & Aqueous \\
\hline Temperature, $\mathrm{C}$ & 25 & 25 & 25 & 25 & 25 & 25 & 25 & 25 & 25 \\
\hline Pressure, atm & 1 & 1 & 1 & 1 & 1 & 1 & 1 & 1 & 1 \\
\hline $\mathrm{pH}$ & 11.9511 & 11.9511 & 11.9511 & 11.9511 & 11.9511 & 11.9511 & 11.9511 & 11.9511 & 11.9511 \\
\hline Total mol $/ \mathrm{hr}$ & 55.346 & 27.673 & 27.673 & 5.5346 & 5.5346 & 5.5346 & 5.5346 & 5.5346 & 5.5346 \\
\hline Flow Units & $\mathrm{g} / \mathrm{hr}$ & $\mathrm{g} / \mathrm{hr}$ & $\mathrm{g} / \mathrm{hr}$ & $\mathrm{g} / \mathrm{hr}$ & $\mathrm{g} / \mathrm{hr}$ & $\mathrm{g} / \mathrm{hr}$ & $\mathrm{g} / \mathrm{hr}$ & $\mathrm{g} / \mathrm{hr}$ & $\mathrm{g} / \mathrm{hr}$ \\
\hline \multicolumn{10}{|l|}{$\begin{array}{c}\text { Acetic Acid, Dimer } \\
\text { Acetic Acid }\end{array}$} \\
\hline Acetic Acid & & & & & & & & & \\
\hline \multicolumn{10}{|l|}{ Aluminum Oxide } \\
\hline \multicolumn{10}{|l|}{ Aldrin } \\
\hline \multirow{2}{*}{\multicolumn{10}{|c|}{$\begin{array}{c}\text { Aluminum Hydrogen EDTA } \\
\text { Aluminum Hvdroxide }\end{array}$}} \\
\hline \multirow{2}{*}{\multicolumn{7}{|c|}{$\begin{array}{c}\text { Aluminum Hydroxide } \\
\text { Benzene }\end{array}$}} & & & \\
\hline & & & & & & & & & \\
\hline \multicolumn{10}{|l|}{$\begin{array}{c}\text { Bis(2-ethylhexyl)phthalate } \\
\text { (BEHP) }\end{array}$} \\
\hline \multicolumn{10}{|l|}{ Benzo(a)pyrene (BaP) } \\
\hline \multicolumn{10}{|l|}{ Phenol } \\
\hline \multirow{2}{*}{\multicolumn{10}{|c|}{$\begin{array}{c}\text { Calcium Chloride Oxide } \\
\text { Calcium Oxalate }\end{array}$}} \\
\hline & & & & & & & & & \\
\hline \multicolumn{10}{|l|}{ Calcium Oxalate Monohydrate } \\
\hline \multicolumn{10}{|l|}{ Calcium Carbonate } \\
\hline \multicolumn{10}{|l|}{ Calcium Dichromate $(\mathrm{VI})$} \\
\hline \multicolumn{10}{|l|}{ Calcium Citrate } \\
\hline \multirow{2}{*}{\multicolumn{10}{|c|}{$\begin{array}{c}\text { Calcium Fluoride } \\
\text { Calcium Hydroxide }\end{array}$}} \\
\hline & & & & & & & & & \\
\hline \multicolumn{10}{|l|}{ Pentachlorophenol } \\
\hline \multicolumn{10}{|l|}{ Hexachlorobenzene } \\
\hline \multicolumn{10}{|l|}{ Chlorobenzene } \\
\hline 2-Chloronaphthalene & & & & & & & & & \\
\hline Carbon Dioxide & & & & & & & & & \\
\hline Cesium Acetate & & & & & & & & & \\
\hline Cesium Glycolate & & & & & & & & & \\
\hline Dibenz[a,h]anthracene & & & & & & & & & \\
\hline 1,2-dibromoethane & & & & & & & & & \\
\hline Diethyl phthalate & & & & & & & & & \\
\hline Iron (III) Oxalate & & & & & & & & & \\
\hline Iron (III) Hydrogen EDTA & & & & & & & & & \\
\hline Iron (III) Hydroxide & & & & & & & & & \\
\hline Formic Acid, dimer & & & & & & & & & \\
\hline Hydrofluoride, dimer & & & & & & & & & \\
\hline 2,2'-Iminobisacetic acid & & & & & & & & & \\
\hline Water & $9.969 \mathrm{E}+02$ & $4.984 \mathrm{E}+02$ & $4.984 \mathrm{E}+02$ & $9.969 \mathrm{E}+01$ & $9.969 \mathrm{E}+01$ & $9.969 \mathrm{E}+01$ & $9.969 \mathrm{E}+01$ & $9.969 \mathrm{E}+01$ & $9.969 \mathrm{E}+01$ \\
\hline Sulfuric Acid & & & & & & & & & \\
\hline Hydrogen Chloride & & & & & & & & & \\
\hline Formic Acid & & & & & & & & & \\
\hline Hydrofluoric Acid & & & & & & & & & \\
\hline Mercury (II) Chloride & & & & & & & & & \\
\hline Mercury (elemental) & & & & & & & & & \\
\hline Mercury (II) Oxide & & & & & & & & & \\
\hline Nitrous (III) Acid & & & & & & & & & \\
\hline Nitric Acid & & & & & & & & & \\
\hline Hexachlorobutadiene & & & & & & & & & \\
\hline Potassium Citrate & & & & & & & & & \\
\hline Potassium Acetate & & & & & & & & & \\
\hline Potassium Chloride & & & & & & & & & \\
\hline
\end{tabular}


WSRC-TR-2002-00550, Rev. 1

SRT-RPP-2002-0027.

\begin{tabular}{|c|c|c|c|c|c|c|c|c|c|}
\hline Stream & $\begin{array}{c}\text { Total } \\
\text { CWash }\end{array}$ & $\begin{array}{c}\text { FCWash } \\
\text { Total }\end{array}$ & $\begin{array}{l}\text { SCWash } \\
\text { Total }\end{array}$ & FCWash-1 & FCWash-2 & FCWash-3 & FCWash-4 & FCWash-5 & SCWash-1 \\
\hline \multicolumn{10}{|l|}{ Potassium Glycolate } \\
\hline \multicolumn{10}{|l|}{ Potassium Nitrate (VI) } \\
\hline \multicolumn{10}{|l|}{ gamma-BHC (Lindane) } \\
\hline \multicolumn{10}{|l|}{$\begin{array}{l}\text { 4-methyl-2-pentanone } \\
\text { (MIBK) }\end{array}$} \\
\hline \multirow{2}{*}{\multicolumn{10}{|c|}{ Magnesium Oxalate }} \\
\hline Magnesium Dichromate $(\mathrm{V}$ & & & & & & & & & \\
\hline \multicolumn{10}{|l|}{ Magnesium Hydroxide } \\
\hline \multicolumn{10}{|l|}{ Manganese (II) Oxalate } \\
\hline \multicolumn{10}{|l|}{ Manganese (II) Hydroxide } \\
\hline \multicolumn{10}{|l|}{ Nitrogen } \\
\hline \multirow{2}{*}{\multicolumn{10}{|c|}{$\begin{array}{c}\text { Sodium Oxalate } \\
\text { Sodium Chromate }(\mathrm{VI})\end{array}$}} \\
\hline & & & & & & & & & \\
\hline \multicolumn{10}{|l|}{ Sodium Fluoride Sulfate } \\
\hline \multicolumn{10}{|l|}{ Sodium Acetate } \\
\hline \multicolumn{10}{|l|}{ Sodium Glycolate } \\
\hline \multicolumn{10}{|l|}{ Sodium Nitrite } \\
\hline Sodium Hydroxide & $3.999 \mathrm{E}-01$ & $1.999 \mathrm{E}-01$ & $1.999 \mathrm{E}-01$ & $3.999 \mathrm{E}-02$ & $3.999 \mathrm{E}-02$ & $3.999 \mathrm{E}-02$ & $3.999 \mathrm{E}-02$ & $3.999 \mathrm{E}-02$ & $3.999 \mathrm{E}-02$ \\
\hline \multicolumn{10}{|l|}{ Naphthalene } \\
\hline \multicolumn{10}{|l|}{ Sodium Aluminosilicate Gel } \\
\hline \multirow{2}{*}{\multicolumn{10}{|c|}{$\begin{array}{l}\text { Nickel (II) Oxalate } \\
\text { Nickel (II) Hydroxide }\end{array}$}} \\
\hline & & & & & & \multicolumn{4}{|c|}{ Nickel (II) Hydroxide } \\
\hline \multicolumn{10}{|l|}{ Oxygen } \\
\hline \multicolumn{10}{|l|}{ Phosphorus Pentoxide } \\
\hline PYRENE & & & & & & & & & \\
\hline Silicon Dioxide & & & & & & & & & \\
\hline Sulfur Trioxide & & & & & & & & & \\
\hline 1,2,4-trichlorobenzene & & & & & & & & & \\
\hline 1,2,3-trichloropropane & & & & & & & & & \\
\hline TOLUENE & & & & & & & & & \\
\hline Zinc Dichloride Pentoxid & & & & & & & & & \\
\hline Zinc Oxalate & & & & & & & & & \\
\hline Zinc Hydroxide & & & & & & & & & \\
\hline Zirconium EDTA & & & & & & & & & \\
\hline Zirconium Oxide & & & & & & & & & \\
\hline Total $\mathrm{g} / \mathrm{hr}$ & $9.973 \mathrm{E}+02$ & $4.986 \mathrm{E}+02$ & $4.986 \mathrm{E}+02$ & $9.973 \mathrm{E}+01$ & $9.973 \mathrm{E}+01$ & $9.973 \mathrm{E}+01$ & $9.973 \mathrm{E}+01$ & $9.973 \mathrm{E}+01$ & $9.973 \mathrm{E}+01$ \\
\hline Volume, L/hr & $1.000 \mathrm{E}+00$ & $5.000 \mathrm{E}-01$ & $5.000 \mathrm{E}-01$ & $1.000 \mathrm{E}-01$ & $1.000 \mathrm{E}-01$ & $1.000 \mathrm{E}-01$ & $1.000 \mathrm{E}-01$ & $1.000 \mathrm{E}-01$ & $1.000 \mathrm{E}-01$ \\
\hline Enthalpy, cal/hr & $-3.781 \mathrm{E}+06$ & $-1.891 \mathrm{E}+06$ & $-1.891 \mathrm{E}+06$ & $-3.781 \mathrm{E}+05$ & $-3.781 \mathrm{E}+05$ & $-3.781 \mathrm{E}+05$ & $-3.781 \mathrm{E}+05$ & $-3.781 \mathrm{E}+05$ & $-3.781 \mathrm{E}+05$ \\
\hline Vapor fraction & & & & & & & & & \\
\hline Solid fraction & & & & & & & & & \\
\hline Organic fraction & & & & & & & & & \\
\hline Osmotic Pres, atm & $4.741 \mathrm{E}-01$ & $4.741 \mathrm{E}-01$ & $4.741 \mathrm{E}-01$ & $4.741 \mathrm{E}-01$ & $4.741 \mathrm{E}-01$ & $4.741 \mathrm{E}-01$ & 4.741E-01 & $4.741 \mathrm{E}-01$ & $4.741 \mathrm{E}-01$ \\
\hline Redox Pot, volts & & & & & & & & & \\
\hline E-Con, 1/ohm-cm & $2.366 \mathrm{E}-03$ & $2.366 \mathrm{E}-03$ & $2.366 \mathrm{E}-03$ & $2.366 \mathrm{E}-03$ & $2.366 \mathrm{E}-03$ & $2.366 \mathrm{E}-03$ & $2.366 \mathrm{E}-03$ & $2.366 \mathrm{E}-03$ & $2.366 \mathrm{E}-03$ \\
\hline E-Con, cm2/ohm-mol & $2.367 \mathrm{E}+02$ & $2.367 \mathrm{E}+02$ & $2.367 \mathrm{E}+02$ & $2.367 \mathrm{E}+02$ & $2.367 \mathrm{E}+02$ & $2.367 \mathrm{E}+02$ & $2.367 \mathrm{E}+02$ & $2.367 \mathrm{E}+02$ & $2.367 \mathrm{E}+02$ \\
\hline Abs Visc, cP & $8.929 \mathrm{E}-01$ & $8.929 \mathrm{E}-01$ & $8.929 \mathrm{E}-01$ & $8.929 \mathrm{E}-01$ & $8.929 \mathrm{E}-01$ & $8.929 \mathrm{E}-01$ & $8.929 \mathrm{E}-01$ & $8.929 \mathrm{E}-01$ & $8.929 \mathrm{E}-01$ \\
\hline Rel Visc & $1.002 \mathrm{E}+00$ & $1.002 \mathrm{E}+00$ & $1.002 \mathrm{E}+00$ & $1.002 \mathrm{E}+00$ & $1.002 \mathrm{E}+00$ & $1.002 \mathrm{E}+00$ & $1.002 \mathrm{E}+00$ & $1.002 \mathrm{E}+00$ & $1.002 \mathrm{E}+00$ \\
\hline Ionic Strength & $1.003 \mathrm{E}-02$ & $1.003 \mathrm{E}-02$ & $1.003 \mathrm{E}-02$ & $1.003 \mathrm{E}-02$ & $1.003 \mathrm{E}-02$ & $1.003 \mathrm{E}-02$ & \begin{tabular}{|c|}
$1.003 \mathrm{E}-02$ \\
\end{tabular} & $1.003 \mathrm{E}-02$ & $1.003 \mathrm{E}-02$ \\
\hline
\end{tabular}


WSRC-TR-2002-00550, Rev. 1

SRT-RPP-2002-0027.

Table B- 2. Waste Feed Evaporator 5 Stage Model Results for UF 1.35 Density Case (cont'd) ${ }^{8}$

\begin{tabular}{|c|c|c|c|c|c|c|c|c|c|}
\hline Stream & SCWash-2 & SCWash-3 & SCWash-4 & SCWash-5 & $\begin{array}{l}\text { FCWash } \\
\text { Slurry }\end{array}$ & $\begin{array}{l}\text { FCW1 } \\
\text { Liquor }\end{array}$ & $\begin{array}{l}\text { FCW1 } \\
\text { Solids }\end{array}$ & $\begin{array}{l}\text { FCW1 } \\
\text { Recycle }\end{array}$ & $\begin{array}{l}\text { FCW1 } \\
\text { Return }\end{array}$ \\
\hline Phase & Aqueous & Aqueous & Aqueous & Aqueous & Mixed & Aqueous & Solid & Aqueous & Aqueous \\
\hline Temperature, $\mathrm{C}$ & 25 & 25 & 25 & 25 & 25 & 25 & 25 & 25 & 25 \\
\hline Pressure, atm & 1 & 1 & 1 & 1 & 1 & 1 & 1 & 1 & 1 \\
\hline $\mathrm{pH}$ & 11.9511 & 11.9511 & 11.9511 & 11.9511 & 14.0328 & 14.0328 & & 14.0328 & 14.0328 \\
\hline Total mol/hr & 5.5346 & 5.5346 & 5.5346 & 5.5346 & 5677.21 & 5473.3 & 203.912 & 5.44456 & 5467.86 \\
\hline Flow Units & $\mathrm{g} / \mathrm{hr}$ & $\mathrm{g} / \mathrm{hr}$ & $\mathrm{g} / \mathrm{hr}$ & $\mathrm{g} / \mathrm{hr}$ & $\mathrm{g} / \mathrm{hr}$ & $\mathrm{g} / \mathrm{hr}$ & $\mathrm{g} / \mathrm{hr}$ & $\mathrm{g} / \mathrm{hr}$ & $\mathrm{g} / \mathrm{hr}$ \\
\hline \multicolumn{10}{|l|}{ Acetic Acid, Dimer } \\
\hline \multicolumn{10}{|l|}{ Acetic Acid } \\
\hline Aluminum Oxide & & & & & $3.614 \mathrm{E}+02$ & $3.614 \mathrm{E}+02$ & & $3.595 \mathrm{E}-01$ & $3.611 \mathrm{E}+02$ \\
\hline Aldrin & & & & & $4.351 E-04$ & $4.351 E-04$ & & $4.328 E-07$ & $4.346 E-04$ \\
\hline Aluminum Hydrogen EDTA & & & & & $9.067 \mathrm{E}+01$ & $9.067 \mathrm{E}+01$ & & $9.020 \mathrm{E}-02$ & $9.058 \mathrm{E}+01$ \\
\hline \multicolumn{10}{|l|}{ Aluminum Hydroxide } \\
\hline Benzene & & & & & $2.136 E-06$ & $2.136 E-06$ & & $2.125 E-09$ & $2.134 E-06$ \\
\hline $\begin{array}{c}\text { Bis(2-ethylhexyl)phthalate } \\
\text { (BEHP) }\end{array}$ & & & & & $5.760 E-03$ & $5.760 E-03$ & & $5.730 E-06$ & $5.755 E-03$ \\
\hline Benzo(a)pyrene (BaP) & & & & & $4.181 E+00$ & $4.735 E-05$ & $4.181 E+00$ & $4.710 E-08$ & $4.730 E-05$ \\
\hline Phenol & & & & & $3.239 E-01$ & $3.239 E-01$ & & $3.222 E-04$ & $3.236 E-01$ \\
\hline \multicolumn{10}{|l|}{ Calcium Chloride Oxide } \\
\hline Calcium Oxalate & & & & & $4.779 \mathrm{E}+01$ & $4.778 \mathrm{E}+01$ & & $4.753 \mathrm{E}-02$ & $4.774 \mathrm{E}+01$ \\
\hline \multicolumn{10}{|l|}{ Calcium Oxalate Monohydrate } \\
\hline \multicolumn{10}{|l|}{ Calcium Carbonate } \\
\hline \multicolumn{10}{|l|}{ Calcium Dichromate (VI) } \\
\hline \multicolumn{10}{|l|}{ Calcium Citrate } \\
\hline Calcium Fluoride & & & & & $1.408 \mathrm{E}+03$ & & $1.408 \mathrm{E}+03$ & & \\
\hline \multicolumn{10}{|l|}{ Calcium Hydroxide } \\
\hline Pentachlorophenol & & & & & $7.012 E-03$ & $7.012 E-03$ & & $6.975 E-06$ & $7.005 E-03$ \\
\hline Hexachlorobenzene & & & & & $4.186 E+00$ & $1.130 E-04$ & $4.186 E+00$ & $1.124 E-07$ & $1.129 E-04$ \\
\hline Chlorobenzene & & & & & $4.581 E-06$ & $4.581 E-06$ & & $4.557 E-09$ & $4.577 E-06$ \\
\hline 2-Chloronaphthalene & & & & & $1.231 E-05$ & $1.231 E-05$ & & $1.225 E-08$ & $1.230 E-05$ \\
\hline Carbon Dioxide & & & & & $6.065 \mathrm{E}+03$ & $6.065 \mathrm{E}+03$ & & $6.033 \mathrm{E}+00$ & $6.059 \mathrm{E}+03$ \\
\hline Cesium Acetate & & & & & $1.524 \mathrm{E}+01$ & $1.524 \mathrm{E}+01$ & & $1.516 \mathrm{E}-02$ & $1.523 \mathrm{E}+01$ \\
\hline \multicolumn{10}{|l|}{ Cesium Glycolate } \\
\hline Dibenz[a,h]anthracene & & & & & $3.140 E-03$ & $3.140 E-03$ & & $3.123 E-06$ & $3.137 E-03$ \\
\hline 1,2-dibromoethane & & & & & $1.101 E-04$ & $1.101 E-04$ & & $1.095 E-07$ & $1.100 E-04$ \\
\hline Diethyl phthalate & & & & & $1.985 E-02$ & $1.985 E-02$ & & $1.975 E-05$ & $1.983 E-02$ \\
\hline \multicolumn{10}{|l|}{ Iron (III) Oxalate } \\
\hline Iron (III) Hydrogen EDTA & & & & & $4.574 \mathrm{E}+01$ & $4.574 \mathrm{E}+01$ & & $4.550 \mathrm{E}-02$ & $4.570 \mathrm{E}+01$ \\
\hline \multicolumn{10}{|l|}{ Iron (III) Hydroxide } \\
\hline \multicolumn{10}{|l|}{ Formic Acid, dimer } \\
\hline \multicolumn{10}{|l|}{ Hydrofluoride, dimer } \\
\hline 2,2'-Iminobisacetic acid & & & & & $8.752 \mathrm{E}+01$ & $8.752 \mathrm{E}+01$ & & $8.706 \mathrm{E}-02$ & $8.743 \mathrm{E}+01$ \\
\hline Water & $9.969 \mathrm{E}+01$ & $9.969 \mathrm{E}+01$ & $9.969 \mathrm{E}+01$ & $9.969 \mathrm{E}+01$ & $7.649 \mathrm{E}+04$ & $7.649 \mathrm{E}+04$ & & $7.609 \mathrm{E}+01$ & $7.641 \mathrm{E}+04$ \\
\hline \multicolumn{10}{|l|}{ Sulfuric Acid } \\
\hline Hydrogen Chloride & & & & & $7.945 \mathrm{E}+02$ & $7.945 \mathrm{E}+02$ & & $7.904 \mathrm{E}-01$ & $7.937 \mathrm{E}+02$ \\
\hline Formic Acid & & & & & $3.061 \mathrm{E}+01$ & $3.061 \mathrm{E}+01$ & & $3.045 \mathrm{E}-02$ & $3.058 \mathrm{E}+01$ \\
\hline Hydrofluoric Acid & & & & & $1.030 \mathrm{E}+02$ & $1.030 \mathrm{E}+02$ & & $1.024 \mathrm{E}-01$ & $1.029 \mathrm{E}+02$ \\
\hline \multicolumn{10}{|l|}{ Mercury (II) Chloride } \\
\hline Mercury (elemental) & & & & & $1.053 \mathrm{E}-05$ & $1.053 \mathrm{E}-05$ & & $1.048 \mathrm{E}-08$ & $1.052 \mathrm{E}-05$ \\
\hline Mercury (II) Oxide & & & & & $5.084 \mathrm{E}+01$ & $1.483 \mathrm{E}+00$ & $4.936 \mathrm{E}+01$ & $1.475 \mathrm{E}-03$ & $1.482 \mathrm{E}+00$ \\
\hline Nitrous (III) Acid & & & & & $7.120 \mathrm{E}+03$ & $7.120 \mathrm{E}+03$ & & $7.083 \mathrm{E}+00$ & $7.113 \mathrm{E}+03$ \\
\hline Nitric Acid & & & & & $5.077 \mathrm{E}+03$ & $5.077 \mathrm{E}+03$ & & $5.050 \mathrm{E}+00$ & $5.072 \mathrm{E}+03$ \\
\hline Hexachlorobutadiene & & & & & $9.605 E-08$ & $9.605 E-08$ & & $9.554 E-11$ & $9.595 E-08$ \\
\hline Potassium Citrate & & & & & $2.182 \mathrm{E}+02$ & $2.182 \mathrm{E}+02$ & & $2.171 \mathrm{E}-01$ & $2.180 \mathrm{E}+02$ \\
\hline Potassium Acetate & & & & & & & & & \\
\hline Potassium Chloride & & & & & $2.887 \mathrm{E}+03$ & $2.887 \mathrm{E}+03$ & & $2.872 \mathrm{E}+00$ & $2.884 \mathrm{E}+03$ \\
\hline
\end{tabular}


WSRC-TR-2002-00550, Rev. 1 SRT-RPP-2002-0027.

\begin{tabular}{|c|c|c|c|c|c|c|c|c|c|}
\hline \\
\hline \multicolumn{10}{|l|}{ Potassium Nitrate (VI) } \\
\hline gamma-BHC (Lindane) & & & & & $2.682 E-04$ & $2.682 E-04$ & & $2.668 E-07$ & $2.680 E-04$ \\
\hline $\begin{array}{c}\text { 4-methyl-2-pentanone } \\
\text { (MIBK) }\end{array}$ & & & & & $5.109 E-05$ & $5.109 E-05$ & & $5.082 E-08$ & $5.104 E-05$ \\
\hline Magnesium Oxalate & & & & & $1.571 \mathrm{E}-04$ & $1.571 \mathrm{E}-04$ & & $1.563 \mathrm{E}-07$ & $1.569 \mathrm{E}-04$ \\
\hline \multicolumn{10}{|l|}{ Magnesium Dichromate (VI) } \\
\hline Magnesium Hydroxide & & & & & $2.392 \mathrm{E}+02$ & & $2.392 \mathrm{E}+02$ & & \\
\hline Manganese (II) Oxalate & & & & & $2.044 \mathrm{E}-01$ & $2.044 \mathrm{E}-01$ & & $2.033 \mathrm{E}-04$ & $2.042 \mathrm{E}-01$ \\
\hline \multicolumn{10}{|l|}{ Manganese (II) Hydroxide } \\
\hline Nitrogen & & & & & $1.053 \mathrm{E}-05$ & $1.053 \mathrm{E}-05$ & & $1.047 \mathrm{E}-08$ & $1.052 \mathrm{E}-05$ \\
\hline Sodium Oxalate & & & & & $1.191 \mathrm{E}+04$ & $1.227 \mathrm{E}+02$ & $1.178 \mathrm{E}+04$ & $1.221 \mathrm{E}-01$ & $1.226 \mathrm{E}+02$ \\
\hline Sodium Chromate (VI) & & & & & $6.453 \mathrm{E}+02$ & $6.453 \mathrm{E}+02$ & & $6.419 \mathrm{E}-01$ & $6.447 \mathrm{E}+02$ \\
\hline Sodium Fluoride Sulfate & & & & & $1.662 \mathrm{E}+04$ & & $1.662 \mathrm{E}+04$ & & \\
\hline Sodium Acetate & & & & & $1.418 \mathrm{E}+01$ & $1.418 \mathrm{E}+01$ & & $1.411 \mathrm{E}-02$ & $1.417 \mathrm{E}+01$ \\
\hline Sodium Glycolate & & & & & $3.522 \mathrm{E}+02$ & $3.522 \mathrm{E}+02$ & & $3.503 \mathrm{E}-01$ & $3.518 \mathrm{E}+02$ \\
\hline Sodium Nitrite & & & & & $5.704 \mathrm{E}+03$ & $5.704 \mathrm{E}+03$ & & $5.674 \mathrm{E}+00$ & $5.698 \mathrm{E}+03$ \\
\hline Sodium Hydroxide & $3.999 \mathrm{E}-02$ & $3.999 \mathrm{E}-02$ & $3.999 \mathrm{E}-02$ & $3.999 \mathrm{E}-02$ & $2.618 \mathrm{E}+04$ & $2.618 \mathrm{E}+04$ & & $2.604 \mathrm{E}+01$ & $2.615 \mathrm{E}+04$ \\
\hline Naphthalene & & & & & $1.354 E-05$ & $1.354 E-05$ & & $1.347 E-08$ & $1.353 E-05$ \\
\hline Sodium Aluminosilicate Gel & & & & & $3.784 \mathrm{E}+03$ & & $3.784 \mathrm{E}+03$ & & \\
\hline Nickel (II) Oxalate & & & & & $6.164 \mathrm{E}-01$ & $6.164 \mathrm{E}-01$ & & $6.132 \mathrm{E}-04$ & $6.158 \mathrm{E}-01$ \\
\hline \multicolumn{10}{|l|}{ Nickel (II) Hydroxide } \\
\hline Oxygen & & & & & $5.856 \mathrm{E}-06$ & $5.856 \mathrm{E}-06$ & & $5.826 \mathrm{E}-09$ & $5.851 \mathrm{E}-06$ \\
\hline Phosphorus Pentoxide & & & & & $4.940 \mathrm{E}+01$ & $4.940 \mathrm{E}+01$ & & $4.914 \mathrm{E}-02$ & $4.935 \mathrm{E}+01$ \\
\hline PYRENE & & & & & $4.225 E-04$ & $4.225 E-04$ & & $4.203 E-07$ & $4.221 E-04$ \\
\hline Silicon Dioxide & & & & & $1.143 \mathrm{E}+01$ & $1.143 \mathrm{E}+01$ & & $1.137 \mathrm{E}-02$ & $1.142 \mathrm{E}+01$ \\
\hline Sulfur Trioxide & & & & & $3.090 \mathrm{E}+03$ & $3.090 \mathrm{E}+03$ & & $3.074 \mathrm{E}+00$ & $3.087 \mathrm{E}+03$ \\
\hline 1,2,4-trichlorobenzene & & & & & $2.859 E-06$ & $2.859 E-06$ & & $2.844 E-09$ & $2.857 E-06$ \\
\hline 1,2,3-trichloropropane & & & & & $2.016 E-05$ & $2.016 E-05$ & & $2.005 E-08$ & $2.014 E-05$ \\
\hline TOLUENE & & & & & $1.776 E-06$ & $1.776 E-06$ & & $1.766 E-09$ & $1.774 E-06$ \\
\hline \multicolumn{10}{|l|}{ Zinc Dichloride Pentoxide } \\
\hline Zinc Oxalate & & & & & $8.805 \mathrm{E}+01$ & $8.805 \mathrm{E}+01$ & & $8.759 \mathrm{E}-02$ & $8.796 \mathrm{E}+01$ \\
\hline \multicolumn{10}{|l|}{ Zinc Hydroxide } \\
\hline Zirconium EDTA & & & & & $2.364 \mathrm{E}+00$ & $2.364 \mathrm{E}+00$ & & $2.351 \mathrm{E}-03$ & $2.361 \mathrm{E}+00$ \\
\hline \multicolumn{10}{|l|}{ Zirconium Oxide } \\
\hline Total g/hr & $9.973 \mathrm{E}+01$ & $9.973 \mathrm{E}+01$ & $9.973 \mathrm{E}+01$ & $9.973 \mathrm{E}+01$ & $1.696 \mathrm{E}+05$ & $1.357 \mathrm{E}+05$ & $3.389 \mathrm{E}+04$ & $1.350 \mathrm{E}+02$ & $1.356 \mathrm{E}+05$ \\
\hline Volume, L/hr & $1.000 \mathrm{E}-01$ & $1.000 \mathrm{E}-01$ & $1.000 \mathrm{E}-01$ & $1.000 \mathrm{E}-01$ & $1.040 \mathrm{E}+02$ & $1.005 \mathrm{E}+02$ & $3.430 \mathrm{E}+00$ & $1.000 \mathrm{E}-01$ & $1.004 \mathrm{E}+02$ \\
\hline Enthalpy, cal/hr & $-3.781 \mathrm{E}+05$ & $-3.781 \mathrm{E}+05$ & $-3.781 \mathrm{E}+05$ & $-3.781 \mathrm{E}+05$ & $-5.053 \mathrm{E}+08$ & $-4.149 \mathrm{E}+08$ & $-9.038 \mathrm{E}+07$ & $-4.127 \mathrm{E}+05$ & $-4.145 \mathrm{E}+08$ \\
\hline \multicolumn{10}{|l|}{ Vapor fraction } \\
\hline Solid fraction & & & & & $3.592 \mathrm{E}-02$ & & $1.000 \mathrm{E}+00$ & & \\
\hline \multicolumn{10}{|l|}{ Organic fraction } \\
\hline Osmotic Pres, atm & $4.741 \mathrm{E}-01$ & $4.741 \mathrm{E}-01$ & 4.741E-01 & $4.741 \mathrm{E}-01$ & $2.713 \mathrm{E}+02$ & $2.713 \mathrm{E}+02$ & & $2.713 \mathrm{E}+02$ & $2.713 \mathrm{E}+02$ \\
\hline \multicolumn{10}{|l|}{ Redox Pot, volts } \\
\hline E-Con, 1/ohm-cm & $2.366 \mathrm{E}-03$ & $2.366 \mathrm{E}-03$ & $2.366 \mathrm{E}-03$ & $2.366 \mathrm{E}-03$ & $2.595 \mathrm{E}-01$ & $2.595 \mathrm{E}-01$ & & $2.595 \mathrm{E}-01$ & $2.595 \mathrm{E}-01$ \\
\hline E-Con, cm2/ohm-mol & $2.367 \mathrm{E}+02$ & $2.367 \mathrm{E}+02$ & $2.367 \mathrm{E}+02$ & $2.367 \mathrm{E}+02$ & $1.823 \mathrm{E}+01$ & $1.823 \mathrm{E}+01$ & & $1.823 \mathrm{E}+01$ & $1.823 \mathrm{E}+01$ \\
\hline Abs Visc, cP & $8.929 \mathrm{E}-01$ & $8.929 \mathrm{E}-01$ & $8.929 \mathrm{E}-01$ & $8.929 \mathrm{E}-01$ & $4.183 \mathrm{E}+00$ & $4.183 \mathrm{E}+00$ & & $4.183 \mathrm{E}+00$ & $4.183 \mathrm{E}+00$ \\
\hline Rel Visc & $1.002 \mathrm{E}+00$ & $1.002 \mathrm{E}+00$ & $1.002 \mathrm{E}+00$ & $1.002 \mathrm{E}+00$ & $4.696 \mathrm{E}+00$ & $4.696 \mathrm{E}+00$ & & $4.696 \mathrm{E}+00$ & $4.696 \mathrm{E}+00$ \\
\hline Ionic Strength & $1.003 \mathrm{E}-02$ & $1.003 \mathrm{E}-02$ & $1.003 \mathrm{E}-02$ & $1.003 \mathrm{E}-02$ & $9.959 \mathrm{E}+00$ & $9.959 \mathrm{E}+00$ & & $9.959 \mathrm{E}+00$ & $9.959 \mathrm{E}+00$ \\
\hline
\end{tabular}


WSRC-TR-2002-00550, Rev. 1

SRT-RPP-2002-0027.

Table B- 2. Waste Feed Evaporator 5 Stage Model Results for UF 1.35 Density Case (cont'd) ${ }^{8}$

\begin{tabular}{|c|c|c|c|c|c|c|c|c|c|}
\hline Stream & $\begin{array}{l}\text { FCW2 } \\
\text { Slurry }\end{array}$ & $\begin{array}{l}\text { FCW2 } \\
\text { Liquor }\end{array}$ & $\begin{array}{l}\text { FCW2 } \\
\text { Solids }\end{array}$ & $\begin{array}{l}\text { FCW2 } \\
\text { Recycle }\end{array}$ & $\begin{array}{l}\text { FCW2 } \\
\text { Return }\end{array}$ & $\begin{array}{l}\text { FCW3 } \\
\text { Slurry }\end{array}$ & $\begin{array}{l}\text { FCW3 } \\
\text { Liquor }\end{array}$ & $\begin{array}{l}\text { FCW3 } \\
\text { Solids } \\
\end{array}$ & $\begin{array}{l}\text { FCW3 } \\
\text { Recycle }\end{array}$ \\
\hline Phase & Mixed & Aqueous & Solid & Aqueous & Aqueous & Mixed & Aqueous & Solid & Aqueous \\
\hline Temperature, $\mathrm{C}$ & 25 & 25 & 25 & 25 & 25 & 25 & 25 & 25 & 25 \\
\hline Pressure, atm & 1 & 1 & 1 & 1 & 1 & 1 & 1 & 1 & 1 \\
\hline $\mathrm{pH}$ & 14.0316 & 14.0316 & & 14.0316 & 14.0316 & 14.0304 & 14.0304 & & 14.0304 \\
\hline Total mol/hr & 5677.34 & 5473.45 & 203.891 & 5.44478 & 5468 & 5677.46 & 5473.59 & 203.871 & 5.445 \\
\hline Flow Units & $\mathrm{g} / \mathrm{hr}$ & $\mathrm{g} / \mathrm{hr}$ & $\mathrm{g} / \mathrm{hr}$ & $\mathrm{g} / \mathrm{hr}$ & $\mathrm{g} / \mathrm{hr}$ & $\mathrm{g} / \mathrm{hr}$ & $\mathrm{g} / \mathrm{hr}$ & $\mathrm{g} / \mathrm{hr}$ & $\mathrm{g} / \mathrm{hr}$ \\
\hline \multicolumn{10}{|l|}{ Acetic Acid, Dimer } \\
\hline \multicolumn{10}{|l|}{ Acetic Acid } \\
\hline Aluminum Oxide & $3.611 \mathrm{E}+02$ & $3.611 \mathrm{E}+02$ & & $3.592 \mathrm{E}-01$ & $3.607 \mathrm{E}+02$ & $3.607 \mathrm{E}+02$ & $3.607 \mathrm{E}+02$ & & $3.588 \mathrm{E}-01$ \\
\hline Aldrin & $4.346 E-04$ & $4.346 E-04$ & & $4.323 E-07$ & $4.342 E-04$ & $4.342 E-04$ & $4.342 E-04$ & & $4.319 E-07$ \\
\hline Aluminum Hydrogen EDTA & $9.058 \mathrm{E}+01$ & $9.058 \mathrm{E}+01$ & & $9.011 \mathrm{E}-02$ & $9.049 \mathrm{E}+01$ & $9.049 \mathrm{E}+01$ & $9.049 \mathrm{E}+01$ & & $9.002 \mathrm{E}-02$ \\
\hline \multicolumn{10}{|l|}{ Aluminum Hydroxide } \\
\hline Benzene & $2.134 E-06$ & $2.134 E-06$ & & $2.123 E-09$ & $2.132 E-06$ & $2.132 E-06$ & $2.132 E-06$ & & $2.120 E-09$ \\
\hline $\begin{array}{c}\text { Bis(2-ethylhexyl)phthalate } \\
\text { (BEHP) }\end{array}$ & $5.755 E-03$ & $5.755 E-03$ & & $5.725 E-06$ & $5.749 E-03$ & $5.749 E-03$ & $5.749 E-03$ & & $5.719 E-06$ \\
\hline Benzo(a)pyrene (BaP) & $4.181 E+00$ & $4.743 E-05$ & $4.181 E+00$ & $4.718 E-08$ & $4.738 E-05$ & $4.181 E+00$ & $4.751 E-05$ & $4.181 E+00$ & $4.726 E-08$ \\
\hline Phenol & $3.236 E-01$ & $3.236 E-01$ & & $3.219 E-04$ & $3.233 E-01$ & $3.233 E-01$ & $3.233 E-01$ & & $3.216 E-04$ \\
\hline \multicolumn{10}{|l|}{ Calcium Chloride Oxide } \\
\hline Calcium Oxalate & $4.770 \mathrm{E}+01$ & $4.770 \mathrm{E}+01$ & & $4.745 \mathrm{E}-02$ & $4.765 \mathrm{E}+01$ & $4.762 \mathrm{E}+01$ & $4.762 \mathrm{E}+01$ & & 4.737E-02 \\
\hline \multicolumn{10}{|l|}{ Calcium Oxalate Monohydrate } \\
\hline \multicolumn{10}{|l|}{ Calcium Carbonate } \\
\hline \multicolumn{10}{|l|}{ Calcium Dichromate (VI) } \\
\hline \multicolumn{10}{|l|}{ Calcium Citrate } \\
\hline Calcium Fluoride & $1.408 \mathrm{E}+03$ & & $1.408 \mathrm{E}+03$ & & & $1.408 \mathrm{E}+03$ & & $1.408 \mathrm{E}+03$ & \\
\hline \multicolumn{10}{|l|}{ Calcium Hydroxide } \\
\hline Pentachlorophenol & $7.005 E-03$ & $7.005 E-03$ & & $6.968 E-06$ & $6.998 E-03$ & $6.998 E-03$ & $6.998 E-03$ & & $6.961 E-06$ \\
\hline Hexachlorobenzene & $4.186 E+00$ & $1.132 E-04$ & $4.186 E+00$ & $1.126 E-07$ & $1.131 E-04$ & $4.186 E+00$ & $1.134 E-04$ & $4.186 E+00$ & $1.128 E-07$ \\
\hline Chlorobenzene & $4.577 E-06$ & $4.577 E-06$ & & $4.553 E-09$ & $4.572 E-06$ & $4.572 E-06$ & $4.572 E-06$ & & $4.548 E-09$ \\
\hline 2-Chloronaphthalene & $1.230 E-05$ & $1.230 E-05$ & & $1.223 E-08$ & $1.229 E-05$ & $1.229 E-05$ & $1.229 E-05$ & & $1.222 E-08$ \\
\hline Carbon Dioxide & $6.059 \mathrm{E}+03$ & $6.059 \mathrm{E}+03$ & & $6.027 \mathrm{E}+00$ & $6.053 \mathrm{E}+03$ & $6.053 \mathrm{E}+03$ & $6.053 \mathrm{E}+03$ & & $6.021 \mathrm{E}+00$ \\
\hline Cesium Acetate & $1.523 \mathrm{E}+01$ & $1.523 \mathrm{E}+01$ & & $1.515 \mathrm{E}-02$ & $1.521 \mathrm{E}+01$ & $1.521 \mathrm{E}+01$ & $1.521 \mathrm{E}+01$ & & $1.513 \mathrm{E}-02$ \\
\hline \multicolumn{10}{|l|}{ Cesium Glycolate } \\
\hline Dibenz[a,h]anthracene & $3.137 E-03$ & $3.137 E-03$ & & $3.120 E-06$ & $3.134 E-03$ & $3.134 E-03$ & $3.134 E-03$ & & $3.117 E-06$ \\
\hline 1,2-dibromoethane & $1.100 E-04$ & $1.100 E-04$ & & $1.094 E-07$ & $1.099 E-04$ & $1.099 E-04$ & $1.099 E-04$ & & $1.093 E-07$ \\
\hline Diethyl phthalate & $1.983 E-02$ & $1.983 E-02$ & & $1.973 E-05$ & $1.981 E-02$ & $1.981 E-02$ & $1.981 E-02$ & & $1.971 E-05$ \\
\hline \multicolumn{10}{|l|}{ Iron (III) Oxalate } \\
\hline Iron (III) Hydrogen EDTA & $4.570 \mathrm{E}+01$ & $4.570 \mathrm{E}+01$ & & $4.546 \mathrm{E}-02$ & $4.565 \mathrm{E}+01$ & $4.565 \mathrm{E}+01$ & $4.565 \mathrm{E}+01$ & & $4.541 \mathrm{E}-02$ \\
\hline \multicolumn{10}{|l|}{ Iron (III) Hydroxide } \\
\hline \multicolumn{10}{|l|}{ Formic Acid, dimer } \\
\hline \multicolumn{10}{|l|}{ Hydrofluoride, dimer } \\
\hline 2,2'-Iminobisacetic acid & $8.743 \mathrm{E}+01$ & $8.743 \mathrm{E}+01$ & & 8.697E-02 & $8.734 \mathrm{E}+01$ & $8.734 \mathrm{E}+01$ & $8.734 \mathrm{E}+01$ & & 8.689E-02 \\
\hline Water & $7.651 \mathrm{E}+04$ & $7.651 \mathrm{E}+04$ & & $7.611 \mathrm{E}+01$ & $7.644 \mathrm{E}+04$ & $7.654 \mathrm{E}+04$ & $7.654 \mathrm{E}+04$ & & $7.614 \mathrm{E}+01$ \\
\hline \multicolumn{10}{|l|}{ Sulfuric Acid } \\
\hline Hydrogen Chloride & $7.937 \mathrm{E}+02$ & $7.937 \mathrm{E}+02$ & & $7.896 \mathrm{E}-01$ & $7.930 \mathrm{E}+02$ & $7.930 \mathrm{E}+02$ & $7.930 \mathrm{E}+02$ & & $7.888 \mathrm{E}-01$ \\
\hline Formic Acid & $3.058 \mathrm{E}+01$ & $3.058 \mathrm{E}+01$ & & $3.042 \mathrm{E}-02$ & $3.055 \mathrm{E}+01$ & $3.055 \mathrm{E}+01$ & $3.055 \mathrm{E}+01$ & & $3.039 \mathrm{E}-02$ \\
\hline Hydrofluoric Acid & $1.032 \mathrm{E}+02$ & $1.032 \mathrm{E}+02$ & & $1.027 \mathrm{E}-01$ & $1.031 \mathrm{E}+02$ & $1.035 \mathrm{E}+02$ & $1.035 \mathrm{E}+02$ & & $1.030 \mathrm{E}-01$ \\
\hline \multicolumn{10}{|l|}{ Mercury (II) Chloride } \\
\hline Mercury (elemental) & $1.052 \mathrm{E}-05$ & $1.052 \mathrm{E}-05$ & & $1.046 \mathrm{E}-08$ & $1.051 \mathrm{E}-05$ & $1.051 \mathrm{E}-05$ & $1.051 \mathrm{E}-05$ & & $1.045 \mathrm{E}-08$ \\
\hline Mercury (II) Oxide & $5.084 \mathrm{E}+01$ & $1.483 \mathrm{E}+00$ & $4.935 \mathrm{E}+01$ & $1.475 \mathrm{E}-03$ & $1.481 \mathrm{E}+00$ & $5.084 \mathrm{E}+01$ & $1.483 \mathrm{E}+00$ & $4.935 \mathrm{E}+01$ & $1.475 \mathrm{E}-03$ \\
\hline Nitrous (III) Acid & $7.113 \mathrm{E}+03$ & $7.113 \mathrm{E}+03$ & & $7.075 \mathrm{E}+00$ & $7.105 \mathrm{E}+03$ & $7.105 \mathrm{E}+03$ & $7.105 \mathrm{E}+03$ & & $7.068 \mathrm{E}+00$ \\
\hline Nitric Acid & $5.072 \mathrm{E}+03$ & $5.072 \mathrm{E}+03$ & & $5.045 \mathrm{E}+00$ & $5.067 \mathrm{E}+03$ & $5.067 \mathrm{E}+03$ & $5.067 \mathrm{E}+03$ & & $5.040 \mathrm{E}+00$ \\
\hline Hexachlorobutadiene & $9.595 E-08$ & $9.595 E-08$ & & $9.545 E-11$ & $9.586 E-08$ & $9.586 E-08$ & $9.586 E-08$ & & $9.536 E-11$ \\
\hline Potassium Citrate & $2.180 \mathrm{E}+02$ & $2.180 \mathrm{E}+02$ & & $2.168 \mathrm{E}-01$ & $2.178 \mathrm{E}+02$ & $2.178 \mathrm{E}+02$ & $2.178 \mathrm{E}+02$ & & $2.166 \mathrm{E}-01$ \\
\hline \multicolumn{10}{|l|}{ Potassium Acetate } \\
\hline Potassium Chloride & $2.884 \mathrm{E}+03$ & $2.884 \mathrm{E}+03$ & & $2.869 \mathrm{E}+00$ & $2.881 \mathrm{E}+03$ & $2.881 \mathrm{E}+03$ & $2.881 \mathrm{E}+03$ & & $2.866 \mathrm{E}+00$ \\
\hline
\end{tabular}


WSRC-TR-2002-00550, Rev. I SRT-RPP-2002-0027.

\begin{tabular}{|c|c|c|c|c|c|c|c|c|c|}
\hline Stream & $\begin{array}{l}\text { FCW2 } \\
\text { Slurry }\end{array}$ & $\begin{array}{l}\text { FCW2 } \\
\text { Liquor }\end{array}$ & $\begin{array}{l}\text { FCW2 } \\
\text { Solids }\end{array}$ & $\begin{array}{l}\text { FCW2 } \\
\text { Recycle }\end{array}$ & $\begin{array}{l}\text { FCW2 } \\
\text { Return }\end{array}$ & $\begin{array}{l}\text { FCW3 } \\
\text { Slurry }\end{array}$ & $\begin{array}{l}\text { FCW3 } \\
\text { Liquor }\end{array}$ & $\begin{array}{l}\text { FCW3 } \\
\text { Solids }\end{array}$ & $\begin{array}{l}\text { FCW3 } \\
\text { Recycle }\end{array}$ \\
\hline \multicolumn{10}{|l|}{ Potassium Glycolate } \\
\hline \multicolumn{10}{|l|}{ Potassium Nitrate (VI) } \\
\hline gamma-BHC (Lindane) & $2.680 E-04$ & $2.680 E-04$ & & $2.665 E-07$ & $2.677 E-04$ & $2.677 E-04$ & $2.677 E-04$ & & $2.663 E-07$ \\
\hline $\begin{array}{c}\text { 4-methyl-2-pentanone } \\
\text { (MIBK) }\end{array}$ & $5.104 E-05$ & $5.104 E-05$ & & $5.077 E-08$ & $5.099 E-05$ & $5.099 E-05$ & $5.099 E-05$ & & $5.072 E-08$ \\
\hline Magnesium Oxalate & $1.581 \mathrm{E}-04$ & $1.581 \mathrm{E}-04$ & & $1.573 \mathrm{E}-07$ & $1.579 \mathrm{E}-04$ & $1.591 \mathrm{E}-04$ & $1.591 \mathrm{E}-04$ & & $1.583 \mathrm{E}-07$ \\
\hline \multicolumn{10}{|l|}{ Magnesium Dichromate (VI) } \\
\hline Magnesium Hydroxide & $2.392 \mathrm{E}+02$ & & $2.392 \mathrm{E}+02$ & & & $2.392 \mathrm{E}+02$ & & $2.392 \mathrm{E}+02$ & \\
\hline Manganese (II) Oxalate & $2.042 \mathrm{E}-01$ & $2.042 \mathrm{E}-01$ & & $2.031 \mathrm{E}-04$ & $2.040 \mathrm{E}-01$ & $2.040 \mathrm{E}-01$ & $2.040 \mathrm{E}-01$ & & $2.029 \mathrm{E}-04$ \\
\hline \multicolumn{10}{|l|}{ Manganese (II) Hydroxide } \\
\hline Nitrogen & $1.052 \mathrm{E}-05$ & $1.052 \mathrm{E}-05$ & & $1.046 \mathrm{E}-08$ & $1.050 \mathrm{E}-05$ & $1.050 \mathrm{E}-05$ & $1.050 \mathrm{E}-05$ & & $1.045 \mathrm{E}-08$ \\
\hline Sodium Oxalate & $1.191 \mathrm{E}+04$ & $1.230 \mathrm{E}+02$ & $1.178 \mathrm{E}+04$ & $1.223 \mathrm{E}-01$ & $1.228 \mathrm{E}+02$ & $1.191 \mathrm{E}+04$ & $1.232 \mathrm{E}+02$ & $1.178 \mathrm{E}+04$ & $1.226 \mathrm{E}-01$ \\
\hline Sodium Chromate (VI) & $6.447 \mathrm{E}+02$ & $6.447 \mathrm{E}+02$ & & $6.413 \mathrm{E}-01$ & $6.440 \mathrm{E}+02$ & $6.440 \mathrm{E}+02$ & $6.440 \mathrm{E}+02$ & & $6.407 \mathrm{E}-01$ \\
\hline Sodium Fluoride Sulfate & $1.662 \mathrm{E}+04$ & & $1.662 \mathrm{E}+04$ & & & $1.661 \mathrm{E}+04$ & & $1.661 \mathrm{E}+04$ & \\
\hline Sodium Acetate & $1.417 \mathrm{E}+01$ & $1.417 \mathrm{E}+01$ & & $1.410 \mathrm{E}-02$ & $1.416 \mathrm{E}+01$ & $1.416 \mathrm{E}+01$ & $1.416 \mathrm{E}+01$ & & $1.408 \mathrm{E}-02$ \\
\hline Sodium Glycolate & $3.518 \mathrm{E}+02$ & $3.518 \mathrm{E}+02$ & & $3.500 \mathrm{E}-01$ & $3.515 \mathrm{E}+02$ & $3.515 \mathrm{E}+02$ & $3.515 \mathrm{E}+02$ & & $3.496 \mathrm{E}-01$ \\
\hline Sodium Nitrite & $5.699 \mathrm{E}+03$ & $5.699 \mathrm{E}+03$ & & $5.669 \mathrm{E}+00$ & $5.693 \mathrm{E}+03$ & $5.694 \mathrm{E}+03$ & $5.694 \mathrm{E}+03$ & & $5.664 \mathrm{E}+00$ \\
\hline Sodium Hydroxide & $2.615 \mathrm{E}+04$ & $2.615 \mathrm{E}+04$ & & $2.602 \mathrm{E}+01$ & $2.613 \mathrm{E}+04$ & $2.613 \mathrm{E}+04$ & $2.613 \mathrm{E}+04$ & & $2.599 \mathrm{E}+01$ \\
\hline Naphthalene & $1.353 E-05$ & $1.353 E-05$ & & $1.346 E-08$ & $1.352 E-05$ & $1.352 E-05$ & $1.352 E-05$ & & $1.345 E-08$ \\
\hline Sodium Aluminosilicate Gel & $3.784 \mathrm{E}+03$ & & $3.784 \mathrm{E}+03$ & & & $3.784 \mathrm{E}+03$ & & $3.784 \mathrm{E}+03$ & \\
\hline Nickel (II) Oxalate & $6.158 \mathrm{E}-01$ & $6.158 \mathrm{E}-01$ & & $6.126 \mathrm{E}-04$ & $6.152 \mathrm{E}-01$ & $6.152 \mathrm{E}-01$ & $6.152 \mathrm{E}-01$ & & $6.120 \mathrm{E}-04$ \\
\hline \multicolumn{10}{|l|}{ Nickel (II) Hydroxide } \\
\hline Oxygen & $5.851 \mathrm{E}-06$ & $5.851 \mathrm{E}-06$ & & $5.820 \mathrm{E}-09$ & $5.845 \mathrm{E}-06$ & $5.845 \mathrm{E}-06$ & $5.845 \mathrm{E}-06$ & & 5.814E-09 \\
\hline Phosphorus Pentoxide & $4.935 \mathrm{E}+01$ & $4.935 \mathrm{E}+01$ & & $4.909 \mathrm{E}-02$ & $4.930 \mathrm{E}+01$ & $4.930 \mathrm{E}+01$ & $4.930 \mathrm{E}+01$ & & $4.904 \mathrm{E}-02$ \\
\hline PYRENE & $4.221 E-04$ & $4.221 E-04$ & & $4.199 E-07$ & $4.216 E-04$ & $4.216 E-04$ & $4.216 E-04$ & & $4.194 E-07$ \\
\hline Silicon Dioxide & $1.140 \mathrm{E}+01$ & $1.140 \mathrm{E}+01$ & & $1.134 \mathrm{E}-02$ & $1.138 \mathrm{E}+01$ & $1.136 \mathrm{E}+01$ & $1.136 \mathrm{E}+01$ & & $1.130 \mathrm{E}-02$ \\
\hline Sulfur Trioxide & $3.088 \mathrm{E}+03$ & $3.088 \mathrm{E}+03$ & & $3.072 \mathrm{E}+00$ & $3.085 \mathrm{E}+03$ & $3.087 \mathrm{E}+03$ & $3.087 \mathrm{E}+03$ & & $3.071 \mathrm{E}+00$ \\
\hline 1,2,4-trichlorobenzene & $2.857 E-06$ & $2.857 E-06$ & & $2.842 E-09$ & $2.854 E-06$ & $2.854 E-06$ & $2.854 E-06$ & & $2.839 E-09$ \\
\hline 1,2,3-trichloropropane & $2.014 E-05$ & $2.014 E-05$ & & $2.003 E-08$ & $2.012 E-05$ & $2.012 E-05$ & $2.012 E-05$ & & $2.001 E-08$ \\
\hline TOLUENE & $1.774 E-06$ & $1.774 E-06$ & & $1.765 E-09$ & $1.772 E-06$ & $1.772 E-06$ & $1.772 E-06$ & & $1.763 E-09$ \\
\hline \multicolumn{10}{|l|}{ Zinc Dichloride Pentoxide } \\
\hline Zinc Oxalate & $8.796 \mathrm{E}+01$ & $8.796 \mathrm{E}+01$ & & $8.750 \mathrm{E}-02$ & $8.788 \mathrm{E}+01$ & $8.788 \mathrm{E}+01$ & $8.788 \mathrm{E}+01$ & & 8.742E-02 \\
\hline \multicolumn{10}{|l|}{ Zinc Hydroxide } \\
\hline Zirconium EDTA & $2.361 \mathrm{E}+00$ & $2.361 \mathrm{E}+00$ & & $2.349 \mathrm{E}-03$ & $2.359 \mathrm{E}+00$ & $2.359 \mathrm{E}+00$ & $2.359 \mathrm{E}+00$ & & $2.347 \mathrm{E}-03$ \\
\hline \multicolumn{10}{|l|}{ Zirconium Oxide } \\
\hline Total g/hr & $1.696 \mathrm{E}+05$ & $1.357 \mathrm{E}+05$ & $3.389 \mathrm{E}+04$ & $1.350 \mathrm{E}+02$ & $1.355 \mathrm{E}+05$ & $1.695 \mathrm{E}+05$ & $1.356 \mathrm{E}+05$ & $3.389 \mathrm{E}+04$ & $1.349 \mathrm{E}+02$ \\
\hline Volume, L/hr & $1.040 \mathrm{E}+02$ & $1.005 \mathrm{E}+02$ & $3.430 \mathrm{E}+00$ & $1.000 \mathrm{E}-01$ & $1.004 \mathrm{E}+02$ & $1.040 \mathrm{E}+02$ & $1.005 \mathrm{E}+02$ & $3.430 \mathrm{E}+00$ & $1.000 \mathrm{E}-01$ \\
\hline Enthalpy, cal/hr & $-5.052 \mathrm{E}+08$ & $-4.149 \mathrm{E}+08$ & $-9.037 \mathrm{E}+07$ & $-4.127 \mathrm{E}+05$ & $-4.144 \mathrm{E}+08$ & $-5.052 \mathrm{E}+08$ & $-4.148 \mathrm{E}+08$ & $-9.036 \mathrm{E}+07$ & $-4.127 \mathrm{E}+05$ \\
\hline \multicolumn{10}{|l|}{ Vapor fraction } \\
\hline Solid fraction & $3.591 \mathrm{E}-02$ & & $1.000 \mathrm{E}+00$ & & & $3.591 \mathrm{E}-02$ & & $1.000 \mathrm{E}+00$ & \\
\hline \multicolumn{10}{|l|}{ Organic fraction } \\
\hline Osmotic Pres, atm & $2.711 \mathrm{E}+02$ & $2.711 \mathrm{E}+02$ & & $2.711 \mathrm{E}+02$ & $2.711 \mathrm{E}+02$ & $2.709 \mathrm{E}+02$ & $2.709 \mathrm{E}+02$ & & $2.709 \mathrm{E}+02$ \\
\hline \multicolumn{10}{|l|}{ Redox Pot, volts } \\
\hline E-Con, 1/ohm-cm & $2.594 \mathrm{E}-01$ & $2.594 \mathrm{E}-01$ & & $2.594 \mathrm{E}-01$ & $2.594 \mathrm{E}-01$ & $2.593 \mathrm{E}-01$ & $2.593 \mathrm{E}-01$ & & $2.593 \mathrm{E}-01$ \\
\hline E-Con, cm2/ohm-mol & $1.823 \mathrm{E}+01$ & $1.823 \mathrm{E}+01$ & & $1.823 \mathrm{E}+01$ & $1.823 \mathrm{E}+01$ & $1.824 \mathrm{E}+01$ & $1.824 \mathrm{E}+01$ & & $1.824 \mathrm{E}+01$ \\
\hline Abs Visc, cP & $4.178 \mathrm{E}+00$ & $4.178 \mathrm{E}+00$ & & $4.178 \mathrm{E}+00$ & $4.178 \mathrm{E}+00$ & $4.174 \mathrm{E}+00$ & $4.174 \mathrm{E}+00$ & & $4.174 \mathrm{E}+00$ \\
\hline Rel Visc & $4.691 \mathrm{E}+00$ & $4.691 \mathrm{E}+00$ & & $4.691 \mathrm{E}+00$ & $4.691 \mathrm{E}+00$ & $4.686 \mathrm{E}+00$ & $4.686 \mathrm{E}+00$ & & $4.686 \mathrm{E}+00$ \\
\hline Ionic Strength & $9.948 \mathrm{E}+00$ & $9.948 \mathrm{E}+00$ & & $9.948 \mathrm{E}+00$ & $9.948 \mathrm{E}+00$ & $9.936 \mathrm{E}+00$ & $9.936 \mathrm{E}+00$ & & $9.936 \mathrm{E}+00$ \\
\hline
\end{tabular}


WSRC-TR-2002-00550, Rev. 1

SRT-RPP-2002-0027.

Table B- 2. Waste Feed Evaporator 5 Stage Model Results for UF 1.35 Density Case (cont'd) ${ }^{8}$

\begin{tabular}{|c|c|c|c|c|c|c|c|c|c|}
\hline Stream & $\begin{array}{l}\text { FCW3 } \\
\text { Return }\end{array}$ & $\begin{array}{l}\text { FCW4 } \\
\text { Slurry }\end{array}$ & $\begin{array}{l}\text { FCW4 } \\
\text { Liquor }\end{array}$ & $\begin{array}{l}\text { FCW4 } \\
\text { Solids }\end{array}$ & $\begin{array}{l}\text { FCW4 } \\
\text { Recycle }\end{array}$ & $\begin{array}{l}\text { FCW4 } \\
\text { Return }\end{array}$ & $\begin{array}{l}\text { FCWash5 } \\
\text { Slurry }\end{array}$ & $\begin{array}{l}\text { FCW5 } \\
\text { Liquor }\end{array}$ & $\begin{array}{l}\text { FCW5 } \\
\text { Solids }\end{array}$ \\
\hline Phase & Aqueous & Mixed & Aqueous & Solid & Aqueous & Aqueous & Mixed & Aqueous & Solid \\
\hline Temperature, $\mathrm{C}$ & 25 & 25 & 25 & 25 & 25 & 25 & 25 & 25 & 25 \\
\hline Pressure, atm & 1 & 1 & 1 & 1 & 1 & 1 & 1 & 1 & 1 \\
\hline $\mathrm{pH}$ & 14.0304 & 14.0292 & 14.0292 & & 14.0292 & 14.0292 & 14.028 & 14.028 & \\
\hline Total mol/hr & 5468.15 & 5677.59 & 5473.74 & 203.85 & 5.44522 & 5468.29 & 5677.71 & 5473.88 & 203.829 \\
\hline Flow Units & $\mathrm{g} / \mathrm{hr}$ & $\mathrm{g} / \mathrm{hr}$ & $\mathrm{g} / \mathrm{hr}$ & $\mathrm{g} / \mathrm{hr}$ & $\mathrm{g} / \mathrm{hr}$ & $\mathrm{g} / \mathrm{hr}$ & $\mathrm{g} / \mathrm{hr}$ & $\mathrm{g} / \mathrm{hr}$ & $\mathrm{g} / \mathrm{hr}$ \\
\hline \multicolumn{10}{|l|}{ Acetic Acid, Dimer } \\
\hline \multicolumn{10}{|l|}{ Acetic Acid } \\
\hline Aluminum Oxide & $3.603 \mathrm{E}+02$ & $3.603 \mathrm{E}+02$ & $3.603 \mathrm{E}+02$ & & $3.584 \mathrm{E}-01$ & $3.600 \mathrm{E}+02$ & $3.599 \mathrm{E}+02$ & $3.599 \mathrm{E}+02$ & \\
\hline Aldrin & $4.338 E-04$ & $4.338 E-04$ & $4.338 E-04$ & & $4.315 E-07$ & $4.333 E-04$ & $4.333 E-04$ & $4.333 E-04$ & \\
\hline Aluminum Hydrogen EDTA & $9.040 \mathrm{E}+01$ & $9.040 \mathrm{E}+01$ & $9.040 \mathrm{E}+01$ & & $8.993 \mathrm{E}-02$ & $9.031 \mathrm{E}+01$ & $9.031 \mathrm{E}+01$ & $9.031 \mathrm{E}+01$ & \\
\hline \multicolumn{10}{|l|}{ Aluminum Hydroxide } \\
\hline Benzene & $2.129 E-06$ & $2.129 E-06$ & $2.129 E-06$ & & $2.118 E-09$ & $2.127 E-06$ & $2.127 E-06$ & $2.127 E-06$ & \\
\hline $\begin{array}{c}\text { Bis(2-ethylhexyl)phthalate } \\
\text { (BEHP) }\end{array}$ & $5.743 E-03$ & $5.743 E-03$ & $5.743 E-03$ & & $5.713 E-06$ & $5.738 E-03$ & $5.738 E-03$ & $5.738 E-03$ & \\
\hline Benzo(a)pyrene (BaP) & $4.746 E-05$ & $4.181 E+00$ & $4.758 E-05$ & $4.181 E+00$ & $4.734 E-08$ & $4.754 E-05$ & $4.181 E+00$ & $4.766 E-05$ & $4.181 E+00$ \\
\hline Phenol & $3.230 E-01$ & $3.230 E-01$ & $3.230 E-01$ & & $3.213 E-04$ & $3.227 E-01$ & $3.227 E-01$ & $3.227 E-01$ & \\
\hline \multicolumn{10}{|l|}{ Calcium Chloride Oxide } \\
\hline Calcium Oxalate & $4.757 \mathrm{E}+01$ & $4.753 \mathrm{E}+01$ & $4.753 \mathrm{E}+01$ & & $4.728 \mathrm{E}-02$ & $4.749 \mathrm{E}+01$ & $4.745 \mathrm{E}+01$ & $4.745 \mathrm{E}+01$ & \\
\hline \multicolumn{10}{|l|}{ Calcium Oxalate Monohydrate } \\
\hline \multicolumn{10}{|l|}{ Calcium Carbonate } \\
\hline \multicolumn{10}{|l|}{ Calcium Dichromate (VI) } \\
\hline \multicolumn{10}{|l|}{ Calcium Citrate } \\
\hline Calcium Fluoride & & $1.408 \mathrm{E}+03$ & & $1.408 \mathrm{E}+03$ & & & $1.408 \mathrm{E}+03$ & & $1.408 \mathrm{E}+03$ \\
\hline \multicolumn{10}{|l|}{ Calcium Hydroxide } \\
\hline Pentachlorophenol & $6.991 E-03$ & $6.991 E-03$ & $6.991 E-03$ & & $6.954 E-06$ & $6.984 E-03$ & $6.984 E-03$ & $6.984 E-03$ & \\
\hline Hexachlorobenzene & $1.133 E-04$ & $4.186 E+00$ & $1.136 E-04$ & $4.186 E+00$ & $1.130 E-07$ & $1.135 E-04$ & $4.186 E+00$ & $1.138 E-04$ & $4.186 E+00$ \\
\hline Chlorobenzene & $4.568 E-06$ & $4.568 E-06$ & $4.568 E-06$ & & $4.544 E-09$ & $4.563 E-06$ & $4.563 E-06$ & $4.563 E-06$ & \\
\hline 2-Chloronaphthalene & $1.227 E-05$ & $1.227 E-05$ & $1.227 E-05$ & & $1.221 E-08$ & $1.226 E-05$ & $1.226 E-05$ & $1.226 E-05$ & \\
\hline Carbon Dioxide & $6.047 \mathrm{E}+03$ & $6.047 \mathrm{E}+03$ & $6.047 \mathrm{E}+03$ & & $6.016 \mathrm{E}+00$ & $6.041 \mathrm{E}+03$ & $6.041 \mathrm{E}+03$ & $6.041 \mathrm{E}+03$ & \\
\hline Cesium Acetate & $1.520 \mathrm{E}+01$ & $1.520 \mathrm{E}+01$ & $1.520 \mathrm{E}+01$ & & $1.512 \mathrm{E}-02$ & $1.518 \mathrm{E}+01$ & $1.518 \mathrm{E}+01$ & $1.518 \mathrm{E}+01$ & \\
\hline \multicolumn{10}{|l|}{ Cesium Glycolate } \\
\hline Dibenz[a,h]anthracene & $3.131 E-03$ & $3.130 E-03$ & $3.131 E-03$ & & $3.114 E-06$ & $3.127 E-03$ & $3.127 E-03$ & $3.127 E-03$ & \\
\hline 1,2-dibromoethane & $1.098 E-04$ & $1.098 E-04$ & $1.098 E-04$ & & $1.092 E-07$ & $1.097 E-04$ & $1.097 E-04$ & $1.097 E-04$ & \\
\hline Diethyl phthalate & $1.979 E-02$ & $1.979 E-02$ & $1.979 E-02$ & & $1.969 E-05$ & $1.977 E-02$ & $1.977 E-02$ & $1.977 E-02$ & \\
\hline Iron (III) Oxalate & & & & & & & & & \\
\hline Iron (III) Hydrogen EDTA & $4.561 \mathrm{E}+01$ & $4.561 \mathrm{E}+01$ & $4.561 \mathrm{E}+01$ & & $4.537 \mathrm{E}-02$ & $4.556 \mathrm{E}+01$ & $4.556 \mathrm{E}+01$ & $4.556 \mathrm{E}+01$ & \\
\hline \multicolumn{10}{|l|}{ Iron (III) Hydroxide } \\
\hline \multicolumn{10}{|l|}{ Formic Acid, dimer } \\
\hline \multicolumn{10}{|l|}{ Hydrofluoride, dimer } \\
\hline $2,2^{\prime}$-Iminobisacetic acid & $8.726 \mathrm{E}+01$ & $8.726 \mathrm{E}+01$ & $8.726 \mathrm{E}+01$ & & $8.680 \mathrm{E}-02$ & $8.717 \mathrm{E}+01$ & $8.717 \mathrm{E}+01$ & $8.717 \mathrm{E}+01$ & \\
\hline Water & $7.646 \mathrm{E}+04$ & $7.656 \mathrm{E}+04$ & $7.656 \mathrm{E}+04$ & & $7.616 \mathrm{E}+01$ & $7.648 \mathrm{E}+04$ & $7.658 \mathrm{E}+04$ & $7.658 \mathrm{E}+04$ & \\
\hline \multicolumn{10}{|l|}{ Sulfuric Acid } \\
\hline Hydrogen Chloride & $7.922 \mathrm{E}+02$ & $7.922 \mathrm{E}+02$ & $7.922 \mathrm{E}+02$ & & $7.880 \mathrm{E}-01$ & $7.914 \mathrm{E}+02$ & $7.914 \mathrm{E}+02$ & $7.914 \mathrm{E}+02$ & \\
\hline Formic Acid & $3.052 \mathrm{E}+01$ & $3.052 \mathrm{E}+01$ & $3.052 \mathrm{E}+01$ & & $3.036 \mathrm{E}-02$ & $3.049 \mathrm{E}+01$ & $3.049 \mathrm{E}+01$ & $3.049 \mathrm{E}+01$ & \\
\hline Hydrofluoric Acid & $1.034 \mathrm{E}+02$ & $1.037 \mathrm{E}+02$ & $1.037 \mathrm{E}+02$ & & $1.032 \mathrm{E}-01$ & $1.036 \mathrm{E}+02$ & $1.040 \mathrm{E}+02$ & $1.040 \mathrm{E}+02$ & \\
\hline \multicolumn{10}{|l|}{ Mercury (II) Chloride } \\
\hline Mercury (elemental) & $1.050 \mathrm{E}-05$ & $1.050 \mathrm{E}-05$ & $1.050 \mathrm{E}-05$ & & $1.044 \mathrm{E}-08$ & $1.049 \mathrm{E}-05$ & $1.049 \mathrm{E}-05$ & $1.049 \mathrm{E}-05$ & \\
\hline Mercury (II) Oxide & $1.481 \mathrm{E}+00$ & $5.083 \mathrm{E}+01$ & $1.482 \mathrm{E}+00$ & $4.935 \mathrm{E}+01$ & $1.475 \mathrm{E}-03$ & $1.481 \mathrm{E}+00$ & $5.083 \mathrm{E}+01$ & $1.482 \mathrm{E}+00$ & $4.935 \mathrm{E}+01$ \\
\hline Nitrous (III) Acid & $7.098 \mathrm{E}+03$ & $7.098 \mathrm{E}+03$ & $7.098 \mathrm{E}+03$ & & $7.061 \mathrm{E}+00$ & $7.091 \mathrm{E}+03$ & $7.090 \mathrm{E}+03$ & $7.090 \mathrm{E}+03$ & \\
\hline Nitric Acid & $5.062 \mathrm{E}+03$ & $5.062 \mathrm{E}+03$ & $5.062 \mathrm{E}+03$ & & $5.035 \mathrm{E}+00$ & $5.057 \mathrm{E}+03$ & $5.057 \mathrm{E}+03$ & $5.057 \mathrm{E}+03$ & \\
\hline Hexachlorobutadiene & $9.576 E-08$ & $9.576 E-08$ & $9.576 E-08$ & & $9.526 E-11$ & $9.567 E-08$ & $9.567 E-08$ & $9.567 E-08$ & \\
\hline Potassium Citrate & $2.176 \mathrm{E}+02$ & $2.176 \mathrm{E}+02$ & $2.176 \mathrm{E}+02$ & & $2.164 \mathrm{E}-01$ & $2.173 \mathrm{E}+02$ & $2.173 \mathrm{E}+02$ & $2.173 \mathrm{E}+02$ & \\
\hline \multicolumn{10}{|l|}{ Potassium Acetate } \\
\hline Potassium Chloride & $2.878 \mathrm{E}+03$ & $2.878 \mathrm{E}+03$ & $2.878 \mathrm{E}+03$ & & $2.863 \mathrm{E}+00$ & $2.875 \mathrm{E}+03$ & $2.875 \mathrm{E}+03$ & $2.875 \mathrm{E}+03$ & \\
\hline
\end{tabular}


WSRC-TR-2002-00550, Rev. 1 SRT-RPP-2002-0027.

\begin{tabular}{|c|c|c|c|c|c|c|c|c|c|}
\hline Stream & $\begin{array}{l}\text { FCW3 } \\
\text { Return }\end{array}$ & $\begin{array}{l}\text { FCW4 } \\
\text { Slurry }\end{array}$ & $\begin{array}{l}\text { FCW4 } \\
\text { Liquor }\end{array}$ & $\begin{array}{l}\text { FCW4 } \\
\text { Solids }\end{array}$ & $\begin{array}{l}\text { FCW4 } \\
\text { Recycle }\end{array}$ & $\begin{array}{l}\text { FCW4 } \\
\text { Return }\end{array}$ & $\begin{array}{l}\text { FCWash5 } \\
\text { Slurry }\end{array}$ & $\begin{array}{l}\text { FCW5 } \\
\text { Liquor }\end{array}$ & $\begin{array}{l}\text { FCW5 } \\
\text { Solids }\end{array}$ \\
\hline \multicolumn{10}{|l|}{ Potassium Glycolate } \\
\hline \multicolumn{10}{|l|}{ Potassium Nitrate (VI) } \\
\hline gamma-BHC (Lindane) & $2.674 E-04$ & $2.674 E-04$ & $2.674 E-04$ & & $2.660 E-07$ & $2.672 E-04$ & $2.672 E-04$ & $2.672 E-04$ & \\
\hline $\begin{array}{c}\text { 4-methyl-2-pentanone } \\
(\text { MIBK) }\end{array}$ & $5.093 E-05$ & $5.093 E-05$ & $5.094 E-05$ & & $5.067 E-08$ & $5.088 E-05$ & $5.088 E-05$ & $5.088 E-05$ & \\
\hline Magnesium Oxalate & $1.590 \mathrm{E}-04$ & $1.602 \mathrm{E}-04$ & $1.602 \mathrm{E}-04$ & & $1.593 \mathrm{E}-07$ & $1.600 \mathrm{E}-04$ & $1.612 \mathrm{E}-04$ & $1.612 \mathrm{E}-04$ & \\
\hline \multicolumn{10}{|l|}{ Magnesium Dichromate (VI) } \\
\hline Magnesium Hydroxide & & $2.392 \mathrm{E}+02$ & & $2.392 \mathrm{E}+02$ & & & $2.392 \mathrm{E}+02$ & & $2.392 \mathrm{E}+02$ \\
\hline Manganese (II) Oxalate & $2.038 \mathrm{E}-01$ & $2.038 \mathrm{E}-01$ & $2.038 \mathrm{E}-01$ & & $2.027 \mathrm{E}-04$ & $2.036 \mathrm{E}-01$ & $2.036 \mathrm{E}-01$ & $2.036 \mathrm{E}-01$ & \\
\hline \multicolumn{10}{|l|}{ Manganese (II) Hydroxide } \\
\hline Nitrogen & $1.049 \mathrm{E}-05$ & $1.049 \mathrm{E}-05$ & $1.049 \mathrm{E}-05$ & & $1.044 \mathrm{E}-08$ & $1.048 \mathrm{E}-05$ & $1.048 \mathrm{E}-05$ & $1.048 \mathrm{E}-05$ & \\
\hline Sodium Oxalate & $1.231 \mathrm{E}+02$ & $1.191 \mathrm{E}+04$ & $1.235 \mathrm{E}+02$ & $1.178 \mathrm{E}+04$ & $1.228 \mathrm{E}-01$ & $1.234 \mathrm{E}+02$ & $1.191 \mathrm{E}+04$ & $1.237 \mathrm{E}+02$ & $1.178 \mathrm{E}+04$ \\
\hline Sodium Chromate (VI) & $6.434 \mathrm{E}+02$ & $6.434 \mathrm{E}+02$ & $6.434 \mathrm{E}+02$ & & $6.400 \mathrm{E}-01$ & $6.427 \mathrm{E}+02$ & $6.427 \mathrm{E}+02$ & $6.427 \mathrm{E}+02$ & \\
\hline Sodium Fluoride Sulfate & & $1.661 \mathrm{E}+04$ & & $1.661 \mathrm{E}+04$ & & & $1.661 \mathrm{E}+04$ & & $1.661 \mathrm{E}+04$ \\
\hline Sodium Acetate & $1.414 \mathrm{E}+01$ & $1.414 \mathrm{E}+01$ & $1.414 \mathrm{E}+01$ & & $1.407 \mathrm{E}-02$ & $1.413 \mathrm{E}+01$ & $1.413 \mathrm{E}+01$ & $1.413 \mathrm{E}+01$ & \\
\hline Sodium Glycolate & $3.511 \mathrm{E}+02$ & $3.511 \mathrm{E}+02$ & $3.511 \mathrm{E}+02$ & & $3.493 \mathrm{E}-01$ & $3.508 \mathrm{E}+02$ & $3.508 \mathrm{E}+02$ & $3.508 \mathrm{E}+02$ & \\
\hline Sodium Nitrite & $5.688 \mathrm{E}+03$ & $5.689 \mathrm{E}+03$ & $5.689 \mathrm{E}+03$ & & $5.659 \mathrm{E}+00$ & $5.683 \mathrm{E}+03$ & $5.683 \mathrm{E}+03$ & $5.683 \mathrm{E}+03$ & \\
\hline Sodium Hydroxide & $2.610 \mathrm{E}+04$ & $2.610 \mathrm{E}+04$ & $2.610 \mathrm{E}+04$ & & $2.597 \mathrm{E}+01$ & $2.608 \mathrm{E}+04$ & $2.608 \mathrm{E}+04$ & $2.608 \mathrm{E}+04$ & \\
\hline Naphthalene & $1.350 E-05$ & $1.350 E-05$ & $1.350 E-05$ & & $1.343 E-08$ & $1.349 E-05$ & $1.349 E-05$ & $1.349 E-05$ & \\
\hline Sodium Aluminosilicate Gel & & $3.784 \mathrm{E}+03$ & & $3.784 \mathrm{E}+03$ & & & $3.784 \mathrm{E}+03$ & & $3.784 \mathrm{E}+03$ \\
\hline Nickel (II) Oxalate & $6.146 \mathrm{E}-01$ & $6.146 \mathrm{E}-01$ & $6.146 \mathrm{E}-01$ & & $6.114 \mathrm{E}-04$ & $6.140 \mathrm{E}-01$ & $6.140 \mathrm{E}-01$ & $6.140 \mathrm{E}-01$ & \\
\hline \multicolumn{10}{|l|}{ Nickel (II) Hydroxide } \\
\hline Oxygen & 5.839E-06 & $5.839 \mathrm{E}-06$ & $5.839 \mathrm{E}-06$ & & $5.808 \mathrm{E}-09$ & $5.833 \mathrm{E}-06$ & $5.833 \mathrm{E}-06$ & $5.833 \mathrm{E}-06$ & \\
\hline Phosphorus Pentoxide & $4.925 \mathrm{E}+01$ & $4.925 \mathrm{E}+01$ & $4.925 \mathrm{E}+01$ & & $4.900 \mathrm{E}-02$ & $4.920 \mathrm{E}+01$ & $4.920 \mathrm{E}+01$ & $4.920 \mathrm{E}+01$ & \\
\hline PYRENE & $4.212 E-04$ & $4.212 E-04$ & $4.212 E-04$ & & $4.190 E-07$ & $4.208 E-04$ & $4.208 E-04$ & $4.208 E-04$ & \\
\hline Silicon Dioxide & $1.135 \mathrm{E}+01$ & $1.132 \mathrm{E}+01$ & $1.132 \mathrm{E}+01$ & & $1.126 \mathrm{E}-02$ & $1.131 \mathrm{E}+01$ & $1.128 \mathrm{E}+01$ & $1.128 \mathrm{E}+01$ & \\
\hline Sulfur Trioxide & $3.084 \mathrm{E}+03$ & $3.085 \mathrm{E}+03$ & $3.085 \mathrm{E}+03$ & & $3.069 \mathrm{E}+00$ & $3.082 \mathrm{E}+03$ & $3.083 \mathrm{E}+03$ & $3.083 \mathrm{E}+03$ & \\
\hline 1,2,4-trichlorobenzene & $2.851 E-06$ & $2.851 E-06$ & $2.851 E-06$ & & $2.836 E-09$ & $2.848 E-06$ & $2.848 E-06$ & $2.848 E-06$ & \\
\hline 1,2,3-trichloropropane & $2.010 E-05$ & $2.010 E-05$ & $2.010 E-05$ & & $1.999 E-08$ & $2.008 E-05$ & $2.008 E-05$ & $2.008 E-05$ & \\
\hline TOLUENE & $1.770 E-06$ & $1.770 E-06$ & $1.770 E-06$ & & $1.761 E-09$ & $1.769 E-06$ & $1.769 E-06$ & $1.769 E-06$ & \\
\hline \multicolumn{10}{|l|}{ Zinc Dichloride Pentoxide } \\
\hline Zinc Oxalate & $8.779 \mathrm{E}+01$ & $8.779 \mathrm{E}+01$ & $8.779 \mathrm{E}+01$ & & $8.733 \mathrm{E}-02$ & $8.770 \mathrm{E}+01$ & $8.770 \mathrm{E}+01$ & $8.770 \mathrm{E}+01$ & \\
\hline \multicolumn{10}{|l|}{ Zinc Hydroxide } \\
\hline Zirconium EDTA & $2.357 \mathrm{E}+00$ & $2.357 \mathrm{E}+00$ & $2.357 \mathrm{E}+00$ & & $2.344 \mathrm{E}-03$ & $2.354 \mathrm{E}+00$ & $2.354 \mathrm{E}+00$ & $2.354 \mathrm{E}+00$ & \\
\hline \multicolumn{10}{|l|}{ Zirconium Oxide } \\
\hline Total $\mathrm{g} / \mathrm{hr}$ & $1.355 \mathrm{E}+05$ & $1.695 \mathrm{E}+05$ & $1.356 \mathrm{E}+05$ & $3.388 \mathrm{E}+04$ & $1.349 \mathrm{E}+02$ & $1.355 \mathrm{E}+05$ & $1.694 \mathrm{E}+05$ & $1.356 \mathrm{E}+05$ & $3.388 \mathrm{E}+04$ \\
\hline Volume, L/hr & $1.004 \mathrm{E}+02$ & $1.040 \mathrm{E}+02$ & $1.005 \mathrm{E}+02$ & $3.430 \mathrm{E}+00$ & $1.000 \mathrm{E}-01$ & $1.004 \mathrm{E}+02$ & $1.040 \mathrm{E}+02$ & $1.005 \mathrm{E}+02$ & $3.430 \mathrm{E}+00$ \\
\hline Enthalpy, cal/hr & $-4.144 \mathrm{E}+08$ & $-5.052 \mathrm{E}+08$ & $-4.148 \mathrm{E}+08$ & $-9.035 \mathrm{E}+07$ & $-4.126 \mathrm{E}+05$ & $-4.144 \mathrm{E}+08$ & $-5.051 \mathrm{E}+08$ & $-4.148 \mathrm{E}+08$ & $-9.034 \mathrm{E}+07$ \\
\hline \multicolumn{10}{|l|}{ Vapor fraction } \\
\hline Solid fraction & & $3.590 \mathrm{E}-02$ & & $1.000 \mathrm{E}+00$ & & & $3.590 \mathrm{E}-02$ & & $1.000 \mathrm{E}+00$ \\
\hline \multicolumn{10}{|l|}{ Organic fraction } \\
\hline Osmotic Pres, atm & $2.709 \mathrm{E}+02$ & $2.707 \mathrm{E}+02$ & $2.707 \mathrm{E}+02$ & & $2.707 \mathrm{E}+02$ & $2.707 \mathrm{E}+02$ & $2.704 \mathrm{E}+02$ & $2.704 \mathrm{E}+02$ & \\
\hline \multicolumn{10}{|l|}{ Redox Pot, volts } \\
\hline E-Con, 1/ohm-cm & $2.593 \mathrm{E}-01$ & $2.592 \mathrm{E}-01$ & $2.592 \mathrm{E}-01$ & & $2.592 \mathrm{E}-01$ & $2.592 \mathrm{E}-01$ & $2.591 \mathrm{E}-01$ & $2.591 \mathrm{E}-01$ & \\
\hline E-Con, cm2/ohm-mol & $1.824 \mathrm{E}+01$ & $1.825 \mathrm{E}+01$ & $1.825 \mathrm{E}+01$ & & $1.825 \mathrm{E}+01$ & $1.825 \mathrm{E}+01$ & $1.825 \mathrm{E}+01$ & $1.825 \mathrm{E}+01$ & \\
\hline Abs Visc, cP & $4.174 \mathrm{E}+00$ & $4.169 \mathrm{E}+00$ & $4.169 \mathrm{E}+00$ & & $4.169 \mathrm{E}+00$ & $4.169 \mathrm{E}+00$ & $4.165 \mathrm{E}+00$ & $4.165 \mathrm{E}+00$ & \\
\hline Rel Visc & $4.686 \mathrm{E}+00$ & $4.681 \mathrm{E}+00$ & $4.681 \mathrm{E}+00$ & & $4.681 \mathrm{E}+00$ & $4.681 \mathrm{E}+00$ & $4.676 \mathrm{E}+00$ & $4.676 \mathrm{E}+00$ & \\
\hline Ionic Strength & $9.936 \mathrm{E}+00$ & $9.925 \mathrm{E}+00$ & $9.925 \mathrm{E}+00$ & & $9.925 \mathrm{E}+00$ & $9.925 \mathrm{E}+00$ & $9.914 \mathrm{E}+00$ & $9.914 \mathrm{E}+00$ & \\
\hline
\end{tabular}


WSRC-TR-2002-00550, Rev. 1

SRT-RPP-2002-0027.

Table B- 2. Waste Feed Evaporator 5 Stage Model Results for UF 1.35 Density Case (cont'd) ${ }^{8}$

\begin{tabular}{|c|c|c|c|c|c|c|c|c|c|}
\hline Stream & $\begin{array}{l}\text { FCW5 } \\
\text { Recycle }\end{array}$ & $\begin{array}{l}\text { FCW5 } \\
\text { Return }\end{array}$ & Leach-in & Total Leach & $\begin{array}{l}\text { FCWash- } \\
\text { Leach }\end{array}$ & Steam-in & Total Steam & UF2 Filtrate & UF2 Slurry \\
\hline Phase & Aqueous & Aqueous & Aqueous & Aqueous & Mixed & Aqueous & Aqueous & Aqueous & Mixed \\
\hline Temperature, $\mathrm{C}$ & 25 & 25 & 25 & 25 & 25 & 25 & 25 & 25 & 25 \\
\hline Pressure, atm & 1 & 1 & 1 & 1 & 1 & 1 & 1 & 1 & 1 \\
\hline $\mathrm{pH}$ & 14.028 & 14.028 & 17.8006 & 17.8006 & 15.2536 & 6.9969 & 6.9969 & 15.1074 & 15.1074 \\
\hline Total $\mathrm{mol} / \mathrm{hr}$ & 5.44544 & 5468.43 & 62.1856 & 1320.66 & 6985.88 & 55.3332 & 798.187 & 1189.57 & 6588.1 \\
\hline Flow Units & $\mathrm{g} / \mathrm{hr}$ & $\mathrm{g} / \mathrm{hr}$ & $\mathrm{g} / \mathrm{hr}$ & $\mathrm{g} / \mathrm{hr}$ & $\mathrm{g} / \mathrm{hr}$ & $\mathrm{g} / \mathrm{hr}$ & $\mathrm{g} / \mathrm{hr}$ & $\mathrm{g} / \mathrm{hr}$ & $\mathrm{g} / \mathrm{hr}$ \\
\hline \multicolumn{10}{|l|}{ Acetic Acid, Dimer } \\
\hline Acetic Acid & & & & & $1.508 \mathrm{E}+01$ & & & $2.378 \mathrm{E}+00$ & $1.270 \mathrm{E}+01$ \\
\hline Aluminum Oxide & $3.581 \mathrm{E}-01$ & $3.596 \mathrm{E}+02$ & & & $1.061 \mathrm{E}+03$ & & & $1.448 \mathrm{E}+02$ & $7.732 \mathrm{E}+02$ \\
\hline Aldrin & $4.311 E-07$ & $4.329 E-04$ & & & $4.329 E-04$ & & & $6.827 E-05$ & $3.646 E-04$ \\
\hline Aluminum Hydrogen EDTA & $8.984 \mathrm{E}-02$ & $9.022 \mathrm{E}+01$ & & & $9.022 \mathrm{E}+01$ & & & $1.423 \mathrm{E}+01$ & $7.599 \mathrm{E}+01$ \\
\hline \multicolumn{10}{|l|}{ Aluminum Hydroxide } \\
\hline Benzene & $2.116 E-09$ & $2.125 E-06$ & & & $2.125 E-06$ & & & $3.352 E-07$ & $1.790 E-06$ \\
\hline $\begin{array}{c}\text { Bis(2-ethylhexyl)phthalate } \\
\text { (BEHP) }\end{array}$ & $5.708 E-06$ & $5.732 E-03$ & & & $5.732 E-03$ & & & $9.039 E-04$ & $4.828 E-03$ \\
\hline Benzo(a)pyrene (BaP) & $4.741 E-08$ & $4.761 E-05$ & & & $4.181 E+00$ & & & $6.359 E-06$ & $4.181 E+00$ \\
\hline Phenol & $3.210 E-04$ & $3.223 E-01$ & & & $3.223 E-01$ & & & $5.083 E-02$ & $2.715 E-01$ \\
\hline \multicolumn{10}{|l|}{ Calcium Chloride Oxide } \\
\hline Calcium Oxalate & $4.720 \mathrm{E}-02$ & $4.740 \mathrm{E}+01$ & & & & & & & \\
\hline \multicolumn{10}{|l|}{ Calcium Oxalate Monohydrate } \\
\hline \multicolumn{10}{|l|}{ Calcium Carbonate } \\
\hline Calcium Dichromate (VI) & & & & & $9.399 \mathrm{E}+01$ & & & $1.554 \mathrm{E}+01$ & $8.302 \mathrm{E}+01$ \\
\hline \multicolumn{10}{|l|}{ Calcium Citrate } \\
\hline Calcium Fluoride & & & & & & & & & $9.453 \mathrm{E}+01$ \\
\hline Calcium Hydroxide & & & & & $1.337 \mathrm{E}+03$ & & & & $1.246 \mathrm{E}+03$ \\
\hline Pentachlorophenol & $6.948 E-06$ & $6.977 E-03$ & & & $6.977 E-03$ & & & $1.100 E-03$ & $5.877 E-03$ \\
\hline Hexachlorobenzene & $1.132 E-07$ & $1.137 E-04$ & & & $4.186 E+00$ & & & $1.518 E-05$ & $4.186 E+00$ \\
\hline Chlorobenzene & $4.539 E-09$ & $4.558 E-06$ & & & $4.558 E-06$ & & & $7.189 E-07$ & $3.840 E-06$ \\
\hline 2-Chloronaphthalene & $1.220 E-08$ & $1.225 E-05$ & & & $1.225 E-05$ & & & $1.932 E-06$ & $1.032 E-05$ \\
\hline Carbon Dioxide & $6.010 \mathrm{E}+00$ & $6.035 \mathrm{E}+03$ & & & $6.035 \mathrm{E}+03$ & & & $9.518 \mathrm{E}+02$ & $5.083 \mathrm{E}+03$ \\
\hline Cesium Acetate & $1.510 \mathrm{E}-02$ & $1.517 \mathrm{E}+01$ & & & & & & & \\
\hline Cesium Glycolate & & & & & $1.643 \mathrm{E}+01$ & & & $2.591 \mathrm{E}+00$ & $1.384 \mathrm{E}+01$ \\
\hline Dibenz[a,h]anthracene & $3.111 E-06$ & $3.124 E-03$ & & & $3.124 E-03$ & & & $4.927 E-04$ & $2.632 E-03$ \\
\hline 1,2-dibromoethane & $1.091 E-07$ & $1.096 E-04$ & & & $1.096 E-04$ & & & $1.728 E-05$ & $9.228 E-05$ \\
\hline Diethyl phthalate & $1.967 E-05$ & $1.975 E-02$ & & & $1.975 E-02$ & & & $3.115 E-03$ & $1.664 E-02$ \\
\hline \multicolumn{10}{|l|}{ Iron (III) Oxalate } \\
\hline Iron (III) Hydrogen EDTA & $4.532 \mathrm{E}-02$ & $4.552 \mathrm{E}+01$ & & & $4.552 \mathrm{E}+01$ & & & $7.178 \mathrm{E}+00$ & $3.834 \mathrm{E}+01$ \\
\hline \multicolumn{10}{|l|}{ Iron (III) Hydroxide } \\
\hline \multicolumn{10}{|l|}{ Formic Acid, dimer } \\
\hline \multicolumn{10}{|l|}{ Hydrofluoride, dimer } \\
\hline 2,2'-Iminobisacetic acid & $8.672 \mathrm{E}-02$ & $8.708 \mathrm{E}+01$ & & & $8.708 \mathrm{E}+01$ & & & $1.373 \mathrm{E}+01$ & $7.335 \mathrm{E}+01$ \\
\hline Water & $7.618 \mathrm{E}+01$ & $7.651 \mathrm{E}+04$ & $7.780 \mathrm{E}+02$ & $1.652 \mathrm{E}+04$ & $9.260 \mathrm{E}+04$ & $9.968 \mathrm{E}+02$ & $1.438 \mathrm{E}+04$ & $1.684 \mathrm{E}+04$ & $8.996 \mathrm{E}+04$ \\
\hline \multicolumn{10}{|l|}{ Sulfuric Acid } \\
\hline Hydrogen Chloride & $7.873 \mathrm{E}-01$ & $7.906 \mathrm{E}+02$ & & & $9.158 \mathrm{E}+02$ & & & $1.447 \mathrm{E}+02$ & $7.728 \mathrm{E}+02$ \\
\hline Formic Acid & $3.033 \mathrm{E}-02$ & $3.046 \mathrm{E}+01$ & & & $3.046 \mathrm{E}+01$ & & & $4.804 \mathrm{E}+00$ & $2.566 \mathrm{E}+01$ \\
\hline Hydrofluoric Acid & $1.035 \mathrm{E}-01$ & $1.039 \mathrm{E}+02$ & & & $1.376 \mathrm{E}+02$ & & & $2.364 \mathrm{E}+01$ & $1.263 \mathrm{E}+02$ \\
\hline \multicolumn{10}{|l|}{ Mercury (II) Chloride } \\
\hline Mercury (elemental) & $1.043 \mathrm{E}-08$ & $1.048 \mathrm{E}-05$ & & & $1.048 \mathrm{E}-05$ & & & $1.652 \mathrm{E}-06$ & $8.826 \mathrm{E}-06$ \\
\hline Mercury (II) Oxide & $1.474 \mathrm{E}-03$ & $1.481 \mathrm{E}+00$ & & & $5.083 \mathrm{E}+01$ & & & $1.492 \mathrm{E}+00$ & $4.934 \mathrm{E}+01$ \\
\hline Nitrous (III) Acid & $7.053 \mathrm{E}+00$ & $7.083 \mathrm{E}+03$ & & & $1.095 \mathrm{E}+04$ & & & $1.727 \mathrm{E}+03$ & $9.225 \mathrm{E}+03$ \\
\hline Nitric Acid & $5.030 \mathrm{E}+00$ & $5.052 \mathrm{E}+03$ & & & $5.052 \mathrm{E}+03$ & & & $7.967 \mathrm{E}+02$ & $4.255 \mathrm{E}+03$ \\
\hline Hexachlorobutadiene & $9.517 E-11$ & $9.557 E-08$ & & & $9.557 E-08$ & & & $1.507 E-08$ & $8.050 E-08$ \\
\hline Potassium Citrate & $2.162 \mathrm{E}-01$ & $2.171 \mathrm{E}+02$ & & & $2.171 \mathrm{E}+02$ & & & $3.424 \mathrm{E}+01$ & $1.829 \mathrm{E}+02$ \\
\hline \multicolumn{10}{|l|}{ Potassium Acetate } \\
\hline Potassium Chloride & $2.861 \mathrm{E}+00$ & $2.873 \mathrm{E}+03$ & & & $2.612 \mathrm{E}+03$ & & & $4.119 \mathrm{E}+02$ & $2.200 \mathrm{E}+03$ \\
\hline
\end{tabular}


WSRC-TR-2002-00550, Rev. 1

SRT-RPP-2002-0027.

\begin{tabular}{|c|c|c|c|c|c|c|c|c|c|}
\hline Stream & $\begin{array}{l}\text { FCW5 } \\
\text { Recycle }\end{array}$ & $\begin{array}{l}\text { FCW5 } \\
\text { Return }\end{array}$ & Leach-in & Total Leach & $\begin{array}{l}\text { FCWash- } \\
\text { Leach }\end{array}$ & Steam-in & Total Steam & UF2 Filtrate & UF2 Slurry \\
\hline Potassium Glycolate & & & & & $3.990 \mathrm{E}+02$ & & & $6.292 \mathrm{E}+01$ & $3.361 \mathrm{E}+02$ \\
\hline \multicolumn{10}{|l|}{ Potassium Nitrate (VI) } \\
\hline gamma-BHC (Lindane) & $2.658 E-07$ & $2.669 E-04$ & & & $2.669 E-04$ & & & $4.209 E-05$ & $2.248 E-04$ \\
\hline $\begin{array}{c}\text { 4-methyl-2-pentanone } \\
\text { (MIBK) }\end{array}$ & $5.062 E-08$ & $5.083 E-05$ & & & $5.083 E-05$ & & & $8.017 E-06$ & $4.282 E-05$ \\
\hline Magnesium Oxalate & $1.604 \mathrm{E}-07$ & $1.610 \mathrm{E}-04$ & & & & & & & \\
\hline Magnesium Dichromate (VI) & & & & & $3.974 \mathrm{E}-06$ & & & $8.621 \mathrm{E}-07$ & $4.604 \mathrm{E}-06$ \\
\hline Magnesium Hydroxide & & & & & $2.392 \mathrm{E}+02$ & & & & $2.392 \mathrm{E}+02$ \\
\hline Manganese (II) Oxalate & $2.025 \mathrm{E}-04$ & $2.034 \mathrm{E}-01$ & & & $2.034 \mathrm{E}-01$ & & & $3.208 \mathrm{E}-02$ & $1.713 \mathrm{E}-01$ \\
\hline \multicolumn{10}{|l|}{ Manganese (II) Hydroxide } \\
\hline Nitrogen & $1.043 \mathrm{E}-08$ & $1.047 \mathrm{E}-05$ & & & $1.047 \mathrm{E}-05$ & & & $1.652 \mathrm{E}-06$ & $8.822 \mathrm{E}-06$ \\
\hline Sodium Oxalate & $1.231 \mathrm{E}-01$ & $1.236 \mathrm{E}+02$ & & & $1.198 \mathrm{E}+04$ & & & & $1.196 \mathrm{E}+04$ \\
\hline Sodium Chromate (VI) & $6.394 \mathrm{E}-01$ & $6.421 \mathrm{E}+02$ & & & $5.232 \mathrm{E}+02$ & & & $8.160 \mathrm{E}+01$ & $4.358 \mathrm{E}+02$ \\
\hline Sodium Fluoride Sulfate & & & & & $2.294 \mathrm{E}+04$ & & & & $2.238 \mathrm{E}+04$ \\
\hline Sodium Acetate & $1.405 \mathrm{E}-02$ & $1.411 \mathrm{E}+01$ & & & & & & & \\
\hline Sodium Glycolate & $3.489 \mathrm{E}-01$ & $3.504 \mathrm{E}+02$ & & & & & & & \\
\hline Sodium Nitrite & $5.654 \mathrm{E}+00$ & $5.678 \mathrm{E}+03$ & & & & & & & \\
\hline Sodium Hydroxide & $2.594 \mathrm{E}+01$ & $2.605 \mathrm{E}+04$ & $7.599 \mathrm{E}+02$ & $1.614 \mathrm{E}+04$ & $4.198 \mathrm{E}+04$ & & & $6.666 \mathrm{E}+03$ & $3.560 \mathrm{E}+04$ \\
\hline Naphthalene & $1.342 E-08$ & $1.348 E-05$ & & & $1.348 E-05$ & & & $2.125 E-06$ & $1.135 E-05$ \\
\hline Sodium Aluminosilicate Gel & & & & & $1.122 \mathrm{E}+03$ & & & & $1.665 \mathrm{E}+03$ \\
\hline Nickel (II) Oxalate & $6.108 \mathrm{E}-04$ & $6.133 \mathrm{E}-01$ & & & $6.133 \mathrm{E}-01$ & & & $9.673 \mathrm{E}-02$ & $5.166 \mathrm{E}-01$ \\
\hline \multicolumn{10}{|l|}{ Nickel (II) Hydroxide } \\
\hline Oxygen & $5.803 \mathrm{E}-09$ & $5.827 \mathrm{E}-06$ & & & $5.827 \mathrm{E}-06$ & & & $9.190 \mathrm{E}-07$ & $4.908 \mathrm{E}-06$ \\
\hline Phosphorus Pentoxide & $4.895 \mathrm{E}-02$ & $4.915 \mathrm{E}+01$ & & & $4.915 \mathrm{E}+01$ & & & $7.752 \mathrm{E}+00$ & $4.140 \mathrm{E}+01$ \\
\hline PYRENE & $4.186 E-07$ & $4.204 E-04$ & & & $4.204 E-04$ & & & $6.630 E-05$ & $3.541 E-04$ \\
\hline Silicon Dioxide & $1.122 \mathrm{E}-02$ & $1.127 \mathrm{E}+01$ & & & $9.760 \mathrm{E}+02$ & & & $1.229 \mathrm{E}+02$ & $6.562 \mathrm{E}+02$ \\
\hline Sulfur Trioxide & $3.067 \mathrm{E}+00$ & $3.080 \mathrm{E}+03$ & & & $3.269 \mathrm{E}+02$ & & & $8.990 \mathrm{E}+01$ & $4.802 \mathrm{E}+02$ \\
\hline 1,2,4-trichlorobenzene & $2.833 E-09$ & $2.845 E-06$ & & & $2.845 E-06$ & & & $4.487 E-07$ & $2.397 E-06$ \\
\hline 1,2,3-trichloropropane & $1.997 E-08$ & $2.006 E-05$ & & & $2.006 E-05$ & & & $3.163 E-06$ & $1.689 E-05$ \\
\hline TOLUENE & $1.759 E-09$ & $1.767 E-06$ & & & $1.767 E-06$ & & & $2.786 E-07$ & $1.488 E-06$ \\
\hline Zinc Dichloride Pentoxide & & & & & $1.691 \mathrm{E}+01$ & & & $6.270 \mathrm{E}-01$ & $3.349 \mathrm{E}+00$ \\
\hline Zinc Oxalate & $8.725 \mathrm{E}-02$ & $8.761 \mathrm{E}+01$ & & & $5.896 \mathrm{E}+01$ & & & $1.275 \mathrm{E}+01$ & $6.812 \mathrm{E}+01$ \\
\hline \multicolumn{10}{|l|}{ Zinc Hydroxide } \\
\hline Zirconium EDTA & $2.342 \mathrm{E}-03$ & $2.352 \mathrm{E}+00$ & & & $2.352 \mathrm{E}+00$ & & & $3.709 \mathrm{E}-01$ & $1.981 \mathrm{E}+00$ \\
\hline \multicolumn{10}{|l|}{ Zirconium Oxide } \\
\hline Total g/hr & $1.349 \mathrm{E}+02$ & $1.354 \mathrm{E}+05$ & $1.538 \mathrm{E}+03$ & $3.266 \mathrm{E}+04$ & $2.020 \mathrm{E}+05$ & $9.968 \mathrm{E}+02$ & $1.438 \mathrm{E}+04$ & $2.818 \mathrm{E}+04$ & $1.882 \mathrm{E}+05$ \\
\hline Volume, L/hr & $1.000 \mathrm{E}-01$ & $1.004 \mathrm{E}+02$ & $1.001 \mathrm{E}+00$ & $2.126 \mathrm{E}+01$ & $1.215 \mathrm{E}+02$ & $1.000 \mathrm{E}+00$ & $1.443 \mathrm{E}+01$ & $2.111 \mathrm{E}+01$ & $1.147 \mathrm{E}+02$ \\
\hline Enthalpy, cal/hr & $-4.126 \mathrm{E}+05$ & $-4.144 \mathrm{E}+08$ & $-4.956 \mathrm{E}+06$ & $-1.053 \mathrm{E}+08$ & $-6.111 \mathrm{E}+08$ & $-3.780 \mathrm{E}+06$ & $-5.453 \mathrm{E}+07$ & $-8.975 \mathrm{E}+07$ & $-5.760 \mathrm{E}+08$ \\
\hline \multicolumn{10}{|l|}{ Vapor fraction } \\
\hline Solid fraction & & & & & $3.398 \mathrm{E}-02$ & & & & $3.562 \mathrm{E}-02$ \\
\hline \multicolumn{10}{|l|}{ Organic fraction } \\
\hline Osmotic Pres, atm & $2.704 \mathrm{E}+02$ & $2.704 \mathrm{E}+02$ & $4.623 \mathrm{E}+03$ & $4.623 \mathrm{E}+03$ & $5.479 \mathrm{E}+02$ & & & $4.780 \mathrm{E}+02$ & $4.780 \mathrm{E}+02$ \\
\hline \multicolumn{10}{|l|}{ Redox Pot, volts } \\
\hline E-Con, 1/ohm-cm & $2.591 \mathrm{E}-01$ & $2.591 \mathrm{E}-01$ & & & $2.536 \mathrm{E}-01$ & $5.489 \mathrm{E}-08$ & $5.489 \mathrm{E}-08$ & & \\
\hline E-Con, cm2/ohm-mol & $1.825 \mathrm{E}+01$ & $1.825 \mathrm{E}+01$ & & & $1.664 \mathrm{E}+01$ & & & & \\
\hline Abs Visc, cP & $4.165 \mathrm{E}+00$ & $4.165 \mathrm{E}+00$ & $4.904 \mathrm{E}+01$ & $4.904 \mathrm{E}+01$ & $6.515 \mathrm{E}+00$ & $8.907 \mathrm{E}-01$ & $8.907 \mathrm{E}-01$ & & \\
\hline Rel Visc & $4.676 \mathrm{E}+00$ & $4.676 \mathrm{E}+00$ & $5.506 \mathrm{E}+01$ & $5.506 \mathrm{E}+01$ & $7.314 \mathrm{E}+00$ & $1.000 \mathrm{E}+00$ & $1.000 \mathrm{E}+00$ & & \\
\hline Ionic Strength & $9.914 \mathrm{E}+00$ & $9.914 \mathrm{E}+00$ & $2.442 \mathrm{E}+01$ & $2.442 \mathrm{E}+01$ & $1.142 \mathrm{E}+01$ & $1.008 \mathrm{E}-07$ & $1.008 \mathrm{E}-07$ & $1.002 \mathrm{E}+01$ & $1.002 \mathrm{E}+01$ \\
\hline
\end{tabular}


WSRC-TR-2002-00550, Rev. 1

SRT-RPP-2002-0027.

Table B- 2. Waste Feed Evaporator 5 Stage Model Results for UF 1.35 Density Case (cont'd) ${ }^{8}$

\begin{tabular}{|c|c|c|c|c|c|c|c|c|c|}
\hline Stream & $\begin{array}{l}\text { SCWash1 } \\
\text { Slurry }\end{array}$ & $\begin{array}{l}\text { SCW1 } \\
\text { Liquor }\end{array}$ & $\begin{array}{l}\text { SCW1 } \\
\text { Solids }\end{array}$ & $\begin{array}{c}\text { SCW1 } \\
\text { Recycle }\end{array}$ & $\begin{array}{l}\text { SCW1 } \\
\text { Return }\end{array}$ & $\begin{array}{l}\text { SCW2 } \\
\text { Slurry }\end{array}$ & $\begin{array}{l}\text { SCW2 } \\
\text { Liquor }\end{array}$ & $\begin{array}{l}\text { SCW2 } \\
\text { Solids }\end{array}$ & $\begin{array}{c}\text { SCW2 } \\
\text { Recycle }\end{array}$ \\
\hline Phase & Mixed & Aqueous & Solid & Aqueous & Aqueous & Mixed & Aqueous & Solid & Aqueous \\
\hline Temperature, $\mathrm{C}$ & 25 & 25 & 25 & 25 & 25 & 25 & 25 & 25 & 25 \\
\hline Pressure, atm & 1 & 1 & 1 & 1 & 1 & 1 & 1 & 1 & 1 \\
\hline $\mathrm{pH}$ & 15.1064 & 15.1064 & & 15.1064 & 15.1064 & 15.1054 & 15.1054 & & 15.1054 \\
\hline Total $\mathrm{mol} / \mathrm{hr}$ & 6593.64 & 6359.03 & 234.609 & 5.63599 & 6353.39 & 6593.55 & 6358.97 & 234.576 & 5.63605 \\
\hline Flow Units & $\mathrm{g} / \mathrm{hr}$ & $\mathrm{g} / \mathrm{hr}$ & $\mathrm{g} / \mathrm{hr}$ & $\mathrm{g} / \mathrm{hr}$ & $\mathrm{g} / \mathrm{hr}$ & $\mathrm{g} / \mathrm{hr}$ & $\mathrm{g} / \mathrm{hr}$ & $\mathrm{g} / \mathrm{hr}$ & $\mathrm{g} / \mathrm{hr}$ \\
\hline \multicolumn{10}{|l|}{ Acetic Acid, Dimer } \\
\hline Acetic Acid & $1.270 \mathrm{E}+01$ & $1.270 \mathrm{E}+01$ & & $1.126 \mathrm{E}-02$ & $1.269 \mathrm{E}+01$ & $1.269 \mathrm{E}+01$ & $1.269 \mathrm{E}+01$ & & $1.125 \mathrm{E}-02$ \\
\hline Aluminum Oxide & $7.725 \mathrm{E}+02$ & $7.725 \mathrm{E}+02$ & & $6.846 \mathrm{E}-01$ & $7.718 \mathrm{E}+02$ & $7.711 \mathrm{E}+02$ & $7.711 \mathrm{E}+02$ & & $6.834 \mathrm{E}-01$ \\
\hline Aldrin & $3.646 E-04$ & $3.646 E-04$ & & $3.232 E-07$ & $3.643 E-04$ & $3.643 E-04$ & $3.643 E-04$ & & $3.229 E-07$ \\
\hline Aluminum Hydrogen EDTA & $7.599 \mathrm{E}+01$ & $7.599 \mathrm{E}+01$ & & $6.735 \mathrm{E}-02$ & $7.593 \mathrm{E}+01$ & $7.593 \mathrm{E}+01$ & $7.593 \mathrm{E}+01$ & & $6.730 \mathrm{E}-02$ \\
\hline \multicolumn{10}{|l|}{ Aluminum Hydroxide } \\
\hline Benzene & $1.790 E-06$ & $1.790 E-06$ & & $1.587 E-09$ & $1.788 E-06$ & $1.788 E-06$ & $1.788 E-06$ & & $1.585 E-09$ \\
\hline $\begin{array}{c}\text { Bis(2-ethylhexyl)phthalate } \\
\text { (BEHP) }\end{array}$ & $4.828 E-03$ & $4.828 E-03$ & & $4.279 E-06$ & $4.824 E-03$ & $4.824 E-03$ & $4.824 E-03$ & & $4.275 E-06$ \\
\hline Benzo(a)pyrene (BaP) & $4.181 E+00$ & $3.405 E-05$ & $4.181 E+00$ & $3.018 E-08$ & $3.402 E-05$ & $4.181 E+00$ & $3.410 E-05$ & $4.181 E+00$ & $3.023 E-08$ \\
\hline Phenol & $2.715 E-01$ & $2.715 E-01$ & & $2.406 E-04$ & $2.713 E-01$ & $2.713 E-01$ & $2.713 E-01$ & & $2.404 E-04$ \\
\hline \multicolumn{10}{|l|}{ Calcium Chloride Oxide } \\
\hline \multicolumn{10}{|l|}{ Calcium Oxalate } \\
\hline \multicolumn{10}{|l|}{ Calcium Oxalate Monohydrate } \\
\hline \multicolumn{10}{|l|}{ Calcium Carbonate } \\
\hline Calcium Dichromate (VI) & $8.305 \mathrm{E}+01$ & $8.305 \mathrm{E}+01$ & & $7.360 \mathrm{E}-02$ & $8.297 \mathrm{E}+01$ & $8.300 \mathrm{E}+01$ & $8.300 \mathrm{E}+01$ & & $7.356 \mathrm{E}-02$ \\
\hline \multicolumn{10}{|l|}{ Calcium Citrate } \\
\hline Calcium Fluoride & $9.615 \mathrm{E}+01$ & & $9.615 \mathrm{E}+01$ & & & $9.777 \mathrm{E}+01$ & & $9.777 \mathrm{E}+01$ & \\
\hline Calcium Hydroxide & $1.244 \mathrm{E}+03$ & & $1.244 \mathrm{E}+03$ & & & $1.243 \mathrm{E}+03$ & & $1.243 \mathrm{E}+03$ & \\
\hline Pentachlorophenol & $5.877 E-03$ & $5.877 E-03$ & & $5.208 E-06$ & $5.871 E-03$ & $5.871 E-03$ & $5.871 E-03$ & & $5.204 E-06$ \\
\hline Hexachlorobenzene & $4.186 E+00$ & $8.128 E-05$ & $4.186 E+00$ & $7.203 E-08$ & $8.120 E-05$ & $4.186 E+00$ & $8.141 E-05$ & $4.186 E+00$ & $7.216 E-08$ \\
\hline Chlorobenzene & $3.840 E-06$ & $3.840 E-06$ & & $3.403 E-09$ & $3.836 E-06$ & $3.836 E-06$ & $3.836 E-06$ & & $3.400 E-09$ \\
\hline 2-Chloronaphthalene & $1.032 E-05$ & $1.032 E-05$ & & $9.144 E-09$ & $1.031 E-05$ & $1.031 E-05$ & $1.031 E-05$ & & $9.136 E-09$ \\
\hline Carbon Dioxide & $5.083 \mathrm{E}+03$ & $5.083 \mathrm{E}+03$ & & $4.505 \mathrm{E}+00$ & $5.079 \mathrm{E}+03$ & $5.079 \mathrm{E}+03$ & $5.079 \mathrm{E}+03$ & & $4.501 \mathrm{E}+00$ \\
\hline \multicolumn{10}{|l|}{ Cesium Acetate } \\
\hline Cesium Glycolate & $1.384 \mathrm{E}+01$ & $1.384 \mathrm{E}+01$ & & $1.227 \mathrm{E}-02$ & $1.383 \mathrm{E}+01$ & $1.383 \mathrm{E}+01$ & $1.383 \mathrm{E}+01$ & & $1.226 \mathrm{E}-02$ \\
\hline Dibenz[a,h]anthracene & $2.632 E-03$ & $2.632 E-03$ & & $2.332 E-06$ & $2.629 E-03$ & $2.629 E-03$ & $2.629 E-03$ & & $2.330 E-06$ \\
\hline 1,2-dibromoethane & $9.228 E-05$ & $9.228 E-05$ & & $8.179 E-08$ & $9.220 E-05$ & $9.220 E-05$ & $9.220 E-05$ & & $8.172 E-08$ \\
\hline Diethyl phthalate & $1.664 E-02$ & $1.664 E-02$ & & $1.474 E-05$ & $1.662 E-02$ & $1.662 E-02$ & $1.662 E-02$ & & $1.473 E-05$ \\
\hline \multicolumn{10}{|l|}{ Iron (III) Oxalate } \\
\hline Iron (III) Hydrogen EDTA & $3.834 \mathrm{E}+01$ & $3.834 \mathrm{E}+01$ & & $3.398 \mathrm{E}-02$ & $3.830 \mathrm{E}+01$ & $3.830 \mathrm{E}+01$ & $3.830 \mathrm{E}+01$ & & $3.395 \mathrm{E}-02$ \\
\hline \multicolumn{10}{|l|}{ Iron (III) Hydroxide } \\
\hline \multicolumn{10}{|l|}{ Formic Acid, dimer } \\
\hline \multicolumn{10}{|l|}{ Hydrofluoride, dimer } \\
\hline 2,2'-Iminobisacetic acid & $7.335 \mathrm{E}+01$ & $7.335 \mathrm{E}+01$ & & $6.501 \mathrm{E}-02$ & $7.329 \mathrm{E}+01$ & $7.329 \mathrm{E}+01$ & $7.329 \mathrm{E}+01$ & & $6.495 \mathrm{E}-02$ \\
\hline Water & $9.006 \mathrm{E}+04$ & $9.006 \mathrm{E}+04$ & & $7.982 \mathrm{E}+01$ & $8.998 \mathrm{E}+04$ & $9.008 \mathrm{E}+04$ & $9.008 \mathrm{E}+04$ & & $7.984 \mathrm{E}+01$ \\
\hline \multicolumn{10}{|l|}{ Sulfuric Acid } \\
\hline Hydrogen Chloride & $7.728 \mathrm{E}+02$ & $7.728 \mathrm{E}+02$ & & $6.850 \mathrm{E}-01$ & $7.721 \mathrm{E}+02$ & $7.721 \mathrm{E}+02$ & $7.721 \mathrm{E}+02$ & & $6.844 \mathrm{E}-01$ \\
\hline Formic Acid & $2.566 \mathrm{E}+01$ & $2.566 \mathrm{E}+01$ & & $2.274 \mathrm{E}-02$ & $2.563 \mathrm{E}+01$ & $2.563 \mathrm{E}+01$ & $2.563 \mathrm{E}+01$ & & $2.272 \mathrm{E}-02$ \\
\hline Hydrofluoric Acid & $1.261 \mathrm{E}+02$ & $1.261 \mathrm{E}+02$ & & $1.118 \mathrm{E}-01$ & $1.260 \mathrm{E}+02$ & $1.259 \mathrm{E}+02$ & $1.259 \mathrm{E}+02$ & & $1.116 \mathrm{E}-01$ \\
\hline \multicolumn{10}{|l|}{ Mercury (II) Chloride } \\
\hline Mercury (elemental) & $8.826 \mathrm{E}-06$ & $8.826 \mathrm{E}-06$ & & $7.822 \mathrm{E}-09$ & $8.818 \mathrm{E}-06$ & $8.818 \mathrm{E}-06$ & $8.818 \mathrm{E}-06$ & & $7.815 \mathrm{E}-09$ \\
\hline Mercury (II) Oxide & $4.934 \mathrm{E}+01$ & $7.963 \mathrm{E}+00$ & $4.138 \mathrm{E}+01$ & $7.058 \mathrm{E}-03$ & $7.956 \mathrm{E}+00$ & $4.933 \mathrm{E}+01$ & $7.951 \mathrm{E}+00$ & $4.138 \mathrm{E}+01$ & $7.047 \mathrm{E}-03$ \\
\hline Nitrous (III) Acid & $9.225 \mathrm{E}+03$ & $9.225 \mathrm{E}+03$ & & $8.176 \mathrm{E}+00$ & $9.217 \mathrm{E}+03$ & $9.217 \mathrm{E}+03$ & $9.217 \mathrm{E}+03$ & & $8.169 \mathrm{E}+00$ \\
\hline Nitric Acid & $4.255 \mathrm{E}+03$ & $4.255 \mathrm{E}+03$ & & $3.771 \mathrm{E}+00$ & $4.251 \mathrm{E}+03$ & $4.251 \mathrm{E}+03$ & $4.251 \mathrm{E}+03$ & & $3.768 \mathrm{E}+00$ \\
\hline Hexachlorobutadiene & $8.050 E-08$ & $8.050 E-08$ & & $7.135 E-11$ & $8.043 E-08$ & $8.043 E-08$ & $8.043 E-08$ & & $7.128 E-11$ \\
\hline Potassium Citrate & $1.829 \mathrm{E}+02$ & $1.829 \mathrm{E}+02$ & & $1.621 \mathrm{E}-01$ & $1.827 \mathrm{E}+02$ & $1.827 \mathrm{E}+02$ & $1.827 \mathrm{E}+02$ & & $1.619 \mathrm{E}-01$ \\
\hline \multicolumn{10}{|l|}{ Potassium Acetate } \\
\hline Potassium Chloride & $2.200 \mathrm{E}+03$ & $2.200 \mathrm{E}+03$ & & $1.950 \mathrm{E}+00$ & $2.198 \mathrm{E}+03$ & $2.198 \mathrm{E}+03$ & $2.198 \mathrm{E}+03$ & & $1.948 \mathrm{E}+00$ \\
\hline
\end{tabular}


WSRC-TR-2002-00550, Rev. 1

SRT-RPP-2002-0027.

\begin{tabular}{|c|c|c|c|c|c|c|c|c|c|}
\hline Stream & $\begin{array}{c}\text { SCWash1 } \\
\text { Slurry }\end{array}$ & $\begin{array}{l}\text { SCW1 } \\
\text { Liquor }\end{array}$ & $\begin{array}{l}\text { SCW1 } \\
\text { Solids }\end{array}$ & $\begin{array}{c}\text { SCW1 } \\
\text { Recycle }\end{array}$ & $\begin{array}{l}\text { SCW1 } \\
\text { Return }\end{array}$ & $\begin{array}{l}\text { SCW2 } \\
\text { Slurry }\end{array}$ & $\begin{array}{l}\text { SCW2 } \\
\text { Liquor }\end{array}$ & $\begin{array}{l}\text { SCW2 } \\
\text { Solids }\end{array}$ & $\begin{array}{c}\text { SCW2 } \\
\text { Recycle }\end{array}$ \\
\hline Potassium Glycolate & $3.361 \mathrm{E}+02$ & $3.361 \mathrm{E}+02$ & & $2.978 \mathrm{E}-01$ & $3.358 \mathrm{E}+02$ & $3.358 \mathrm{E}+02$ & $3.358 \mathrm{E}+02$ & & $2.976 \mathrm{E}-01$ \\
\hline \multicolumn{10}{|l|}{ Potassium Nitrate (VI) } \\
\hline gamma-BHC (Lindane) & $2.248 E-04$ & $2.248 E-04$ & & $1.992 E-07$ & $2.246 E-04$ & $2.246 E-04$ & $2.246 E-04$ & & $1.991 E-07$ \\
\hline $\begin{array}{c}\text { 4-methyl-2-pentanone } \\
\text { (MIBK) }\end{array}$ & $4.282 E-05$ & $4.282 E-05$ & & $3.795 E-08$ & $4.278 E-05$ & $4.278 E-05$ & $4.278 E-05$ & & $3.792 E-08$ \\
\hline \multicolumn{10}{|l|}{ Magnesium Oxalate } \\
\hline Magnesium Dichromate (VI) & $4.616 \mathrm{E}-06$ & $4.616 \mathrm{E}-06$ & & 4.091E-09 & $4.612 \mathrm{E}-06$ & $4.623 \mathrm{E}-06$ & $4.623 \mathrm{E}-06$ & & 4.097E-09 \\
\hline Magnesium Hydroxide & $2.392 \mathrm{E}+02$ & & $2.392 \mathrm{E}+02$ & & & $2.392 \mathrm{E}+02$ & & $2.392 \mathrm{E}+02$ & \\
\hline Manganese (II) Oxalate & $1.713 \mathrm{E}-01$ & $1.713 \mathrm{E}-01$ & & $1.518 \mathrm{E}-04$ & $1.712 \mathrm{E}-01$ & $1.712 \mathrm{E}-01$ & $1.712 \mathrm{E}-01$ & & $1.517 \mathrm{E}-04$ \\
\hline \multicolumn{10}{|l|}{ Manganese (II) Hydroxide } \\
\hline Nitrogen & $8.822 \mathrm{E}-06$ & $8.822 \mathrm{E}-06$ & & 7.819E-09 & $8.814 \mathrm{E}-06$ & $8.814 \mathrm{E}-06$ & $8.814 \mathrm{E}-06$ & & 7.812E-09 \\
\hline Sodium Oxalate & $1.196 \mathrm{E}+04$ & & $1.196 \mathrm{E}+04$ & & & $1.196 \mathrm{E}+04$ & & $1.196 \mathrm{E}+04$ & \\
\hline Sodium Chromate (VI) & $4.358 \mathrm{E}+02$ & $4.358 \mathrm{E}+02$ & & $3.862 \mathrm{E}-01$ & $4.354 \mathrm{E}+02$ & $4.354 \mathrm{E}+02$ & $4.354 \mathrm{E}+02$ & & $3.859 \mathrm{E}-01$ \\
\hline Sodium Fluoride Sulfate & $2.237 \mathrm{E}+04$ & & $2.237 \mathrm{E}+04$ & & & $2.237 \mathrm{E}+04$ & & $2.237 \mathrm{E}+04$ & \\
\hline \multicolumn{10}{|l|}{ Sodium Acetate } \\
\hline \multicolumn{10}{|l|}{ Sodium Glycolate } \\
\hline \multicolumn{10}{|l|}{ Sodium Nitrite } \\
\hline Sodium Hydroxide & $3.560 \mathrm{E}+04$ & $3.560 \mathrm{E}+04$ & & $3.156 \mathrm{E}+01$ & $3.557 \mathrm{E}+04$ & $3.558 \mathrm{E}+04$ & $3.558 \mathrm{E}+04$ & & $3.153 \mathrm{E}+01$ \\
\hline Naphthalene & $1.135 E-05$ & $1.135 E-05$ & & $1.006 E-08$ & $1.134 E-05$ & $1.134 E-05$ & $1.134 E-05$ & & $1.005 E-08$ \\
\hline Sodium Aluminosilicate Gel & $1.668 \mathrm{E}+03$ & & $1.668 \mathrm{E}+03$ & & & $1.671 \mathrm{E}+03$ & & $1.671 \mathrm{E}+03$ & \\
\hline Nickel (II) Oxalate & $5.166 \mathrm{E}-01$ & $5.166 \mathrm{E}-01$ & & $4.579 \mathrm{E}-04$ & $5.162 \mathrm{E}-01$ & $5.162 \mathrm{E}-01$ & $5.162 \mathrm{E}-01$ & & $4.575 \mathrm{E}-04$ \\
\hline \multicolumn{10}{|l|}{ Nickel (II) Hydroxide } \\
\hline Oxygen & $4.908 \mathrm{E}-06$ & $4.908 \mathrm{E}-06$ & & $4.350 \mathrm{E}-09$ & 4.904E-06 & $4.904 \mathrm{E}-06$ & 4.904E-06 & & $4.346 \mathrm{E}-09$ \\
\hline Phosphorus Pentoxide & $4.140 \mathrm{E}+01$ & $4.140 \mathrm{E}+01$ & & $3.669 \mathrm{E}-02$ & $4.137 \mathrm{E}+01$ & $4.137 \mathrm{E}+01$ & $4.137 \mathrm{E}+01$ & & $3.666 \mathrm{E}-02$ \\
\hline PYRENE & $3.541 E-04$ & $3.541 E-04$ & & $3.138 E-07$ & $3.538 E-04$ & $3.538 E-04$ & $3.538 E-04$ & & $3.136 E-07$ \\
\hline Silicon Dioxide & $6.552 \mathrm{E}+02$ & $6.552 \mathrm{E}+02$ & & 5.807E-01 & $6.546 \mathrm{E}+02$ & $6.537 \mathrm{E}+02$ & $6.537 \mathrm{E}+02$ & & 5.794E-01 \\
\hline Sulfur Trioxide & $4.829 \mathrm{E}+02$ & $4.829 \mathrm{E}+02$ & & $4.280 \mathrm{E}-01$ & $4.825 \mathrm{E}+02$ & $4.852 \mathrm{E}+02$ & $4.852 \mathrm{E}+02$ & & $4.301 \mathrm{E}-01$ \\
\hline 1,2,4-trichlorobenzene & $2.397 E-06$ & $2.397 E-06$ & & $2.124 E-09$ & $2.394 E-06$ & $2.394 E-06$ & $2.394 E-06$ & & $2.122 E-09$ \\
\hline 1,2,3-trichl oropropane & $1.689 E-05$ & $1.689 E-05$ & & $1.497 E-08$ & $1.688 E-05$ & $1.688 E-05$ & $1.688 E-05$ & & $1.496 E-08$ \\
\hline TOLUENE & $1.488 E-06$ & $1.488 E-06$ & & $1.319 E-09$ & $1.487 E-06$ & $1.487 E-06$ & $1.487 E-06$ & & $1.318 E-09$ \\
\hline Zinc Dichloride Pentoxide & $3.257 \mathrm{E}+00$ & $3.257 \mathrm{E}+00$ & & $2.886 \mathrm{E}-03$ & $3.254 \mathrm{E}+00$ & $3.161 \mathrm{E}+00$ & $3.161 \mathrm{E}+00$ & & $2.802 \mathrm{E}-03$ \\
\hline Zinc Oxalate & $6.828 \mathrm{E}+01$ & $6.828 \mathrm{E}+01$ & & $6.052 \mathrm{E}-02$ & $6.822 \mathrm{E}+01$ & $6.838 \mathrm{E}+01$ & $6.838 \mathrm{E}+01$ & & $6.060 \mathrm{E}-02$ \\
\hline \multicolumn{10}{|l|}{ Zinc Hydroxide } \\
\hline Zirconium EDTA & $1.981 \mathrm{E}+00$ & $1.981 \mathrm{E}+00$ & & $1.756 \mathrm{E}-03$ & $1.979 \mathrm{E}+00$ & $1.979 \mathrm{E}+00$ & $1.979 \mathrm{E}+00$ & & $1.754 \mathrm{E}-03$ \\
\hline \multicolumn{10}{|l|}{ Zirconium Oxide } \\
\hline Total g/hr & $1.883 \mathrm{E}+05$ & $1.506 \mathrm{E}+05$ & $3.763 \mathrm{E}+04$ & $1.335 \mathrm{E}+02$ & $1.505 \mathrm{E}+05$ & $1.882 \mathrm{E}+05$ & $1.506 \mathrm{E}+05$ & $3.763 \mathrm{E}+04$ & $1.335 \mathrm{E}+02$ \\
\hline Volume, L/hr & $1.148 \mathrm{E}+02$ & $1.128 \mathrm{E}+02$ & $1.961 \mathrm{E}+00$ & $1.000 \mathrm{E}-01$ & $1.127 \mathrm{E}+02$ & $1.148 \mathrm{E}+02$ & $1.128 \mathrm{E}+02$ & $1.963 \mathrm{E}+00$ & $1.000 \mathrm{E}-01$ \\
\hline Enthalpy, cal/hr & $-5.764 \mathrm{E}+08$ & $-4.797 \mathrm{E}+08$ & $-9.670 \mathrm{E}+07$ & $-4.252 \mathrm{E}+05$ & $-4.793 \mathrm{E}+08$ & $-5.764 \mathrm{E}+08$ & $-4.797 \mathrm{E}+08$ & $-9.670 \mathrm{E}+07$ & $-4.252 \mathrm{E}+05$ \\
\hline \multicolumn{10}{|l|}{ Vapor fraction } \\
\hline Solid fraction & $3.558 \mathrm{E}-02$ & & $1.000 \mathrm{E}+00$ & & & $3.558 \mathrm{E}-02$ & & $1.000 \mathrm{E}+00$ & \\
\hline \multicolumn{10}{|l|}{ Organic fraction } \\
\hline Osmotic Pres, atm & $4.775 \mathrm{E}+02$ & $4.775 \mathrm{E}+02$ & & $4.775 \mathrm{E}+02$ & $4.775 \mathrm{E}+02$ & $4.770 \mathrm{E}+02$ & $4.770 \mathrm{E}+02$ & & $4.770 \mathrm{E}+02$ \\
\hline \multicolumn{10}{|l|}{ Redox Pot, volts } \\
\hline E-Con, 1/ohm-cm & $2.573 \mathrm{E}-01$ & $2.573 \mathrm{E}-01$ & & $2.573 \mathrm{E}-01$ & $2.573 \mathrm{E}-01$ & $2.573 \mathrm{E}-01$ & $2.573 \mathrm{E}-01$ & & $2.573 \mathrm{E}-01$ \\
\hline E-Con, cm2/ohm-mol & $1.821 \mathrm{E}+01$ & $1.821 \mathrm{E}+01$ & & $1.821 \mathrm{E}+01$ & $1.821 \mathrm{E}+01$ & $1.822 \mathrm{E}+01$ & $1.822 \mathrm{E}+01$ & & $1.822 \mathrm{E}+01$ \\
\hline Abs Visc, cP & $5.517 \mathrm{E}+00$ & $5.517 \mathrm{E}+00$ & & $5.517 \mathrm{E}+00$ & $5.517 \mathrm{E}+00$ & $5.510 \mathrm{E}+00$ & $5.510 \mathrm{E}+00$ & & $5.510 \mathrm{E}+00$ \\
\hline Rel Visc & $6.193 \mathrm{E}+00$ & $6.193 \mathrm{E}+00$ & & $6.193 \mathrm{E}+00$ & $6.193 \mathrm{E}+00$ & $6.186 \mathrm{E}+00$ & $6.186 \mathrm{E}+00$ & & $6.186 \mathrm{E}+00$ \\
\hline Ionic Strength & $1.001 \mathrm{E}+01$ & $1.001 \mathrm{E}+01$ & & $1.001 \mathrm{E}+01$ & $1.001 \mathrm{E}+01$ & $1.000 \mathrm{E}+01$ & $1.000 \mathrm{E}+01$ & & $1.000 \mathrm{E}+01$ \\
\hline
\end{tabular}


WSRC-TR-2002-00550, Rev. 1

SRT-RPP-2002-0027.

Table B- 2. Waste Feed Evaporator 5 Stage Model Results for UF 1.35 Density Case (cont'd) ${ }^{8}$

\begin{tabular}{|c|c|c|c|c|c|c|c|c|c|}
\hline Stream & $\begin{array}{l}\text { SCW2 } \\
\text { Return }\end{array}$ & $\begin{array}{l}\text { SCW3 } \\
\text { Slurry }\end{array}$ & $\begin{array}{l}\text { SCW3 } \\
\text { Liquor }\end{array}$ & $\begin{array}{l}\text { SCW3 } \\
\text { Solids }\end{array}$ & $\begin{array}{c}\text { SCW3 } \\
\text { Recycle }\end{array}$ & $\begin{array}{l}\text { SCW3 } \\
\text { Return }\end{array}$ & $\begin{array}{l}\text { SCW4 } \\
\text { Slurry }\end{array}$ & $\begin{array}{l}\text { SCW4 } \\
\text { Liquor }\end{array}$ & $\begin{array}{l}\text { SCW4 } \\
\text { Solids }\end{array}$ \\
\hline Phase & Aqueous & Mixed & Aqueous & Solid & Aqueous & Aqueous & Mixed & Aqueous & Solid \\
\hline Temperature, $\mathrm{C}$ & 25 & 25 & 25 & 25 & 25 & 25 & 25 & 25 & 25 \\
\hline Pressure, atm & 1 & 1 & 1 & 1 & 1 & 1 & 1 & 1 & 1 \\
\hline $\mathrm{pH}$ & 15.1054 & 15.1044 & 15.1044 & & 15.1044 & 15.1044 & 15.1033 & 15.1033 & \\
\hline Total mol/hr & 6353.33 & 6593.45 & 6358.91 & 234.542 & 5.63612 & 6353.27 & 6593.36 & 6358.85 & 234.509 \\
\hline Flow Units & $\mathrm{g} / \mathrm{hr}$ & $\mathrm{g} / \mathrm{hr}$ & $\mathrm{g} / \mathrm{hr}$ & $\mathrm{g} / \mathrm{hr}$ & $\mathrm{g} / \mathrm{hr}$ & $\mathrm{g} / \mathrm{hr}$ & $\mathrm{g} / \mathrm{hr}$ & $\mathrm{g} / \mathrm{hr}$ & $\mathrm{g} / \mathrm{hr}$ \\
\hline \multicolumn{10}{|l|}{ Acetic Acid, Dimer } \\
\hline Acetic Acid & $1.268 \mathrm{E}+01$ & $1.268 \mathrm{E}+01$ & $1.268 \mathrm{E}+01$ & & $1.124 \mathrm{E}-02$ & $1.267 \mathrm{E}+01$ & $1.267 \mathrm{E}+01$ & $1.267 \mathrm{E}+01$ & \\
\hline Aluminum Oxide & $7.704 \mathrm{E}+02$ & $7.697 \mathrm{E}+02$ & $7.697 \mathrm{E}+02$ & & $6.822 \mathrm{E}-01$ & $7.690 \mathrm{E}+02$ & $7.683 \mathrm{E}+02$ & $7.683 \mathrm{E}+02$ & \\
\hline Aldrin & $3.640 E-04$ & $3.640 E-04$ & $3.640 E-04$ & & $3.226 E-07$ & $3.637 E-04$ & $3.637 E-04$ & $3.637 E-04$ & \\
\hline Aluminum Hydrogen EDTA & $7.586 \mathrm{E}+01$ & $7.586 \mathrm{E}+01$ & $7.586 \mathrm{E}+01$ & & $6.724 \mathrm{E}-02$ & $7.579 \mathrm{E}+01$ & $7.579 \mathrm{E}+01$ & $7.579 \mathrm{E}+01$ & \\
\hline \multicolumn{10}{|l|}{ Aluminum Hydroxide } \\
\hline Benzene & $1.787 E-06$ & $1.787 E-06$ & $1.787 E-06$ & & $1.584 E-09$ & $1.785 E-06$ & $1.785 E-06$ & $1.785 E-06$ & \\
\hline $\begin{array}{c}\text { Bis(2-ethylhexyl)phthalate } \\
\text { (BEHP) }\end{array}$ & $4.819 E-03$ & $4.819 E-03$ & $4.819 E-03$ & & $4.272 E-06$ & $4.815 E-03$ & $4.815 E-03$ & $4.815 E-03$ & \\
\hline Benzo(a)pyrene (BaP) & $3.407 E-05$ & $4.181 E+00$ & $3.416 E-05$ & $4.181 E+00$ & $3.028 E-08$ & $3.413 E-05$ & $4.181 E+00$ & $3.422 E-05$ & $4.181 E+00$ \\
\hline Phenol & $2.710 E-01$ & $2.710 E-01$ & $2.710 E-01$ & & $2.402 E-04$ & $2.708 E-01$ & $2.708 E-01$ & $2.708 E-01$ & \\
\hline \multicolumn{10}{|l|}{ Calcium Chloride Oxide } \\
\hline \multicolumn{10}{|l|}{ Calcium Oxalate } \\
\hline \multicolumn{10}{|l|}{ Calcium Oxalate Monohydrate } \\
\hline \multicolumn{10}{|l|}{ Calcium Carbonate } \\
\hline Calcium Dichromate (VI) & $8.292 \mathrm{E}+01$ & $8.295 \mathrm{E}+01$ & $8.295 \mathrm{E}+01$ & & 7.352E-02 & $8.287 \mathrm{E}+01$ & $8.290 \mathrm{E}+01$ & $8.290 \mathrm{E}+01$ & \\
\hline \multicolumn{10}{|l|}{ Calcium Citrate } \\
\hline Calcium Fluoride & & $9.939 \mathrm{E}+01$ & & $9.939 \mathrm{E}+01$ & & & $1.010 \mathrm{E}+02$ & & $1.010 \mathrm{E}+02$ \\
\hline Calcium Hydroxide & & $1.241 \mathrm{E}+03$ & & $1.241 \mathrm{E}+03$ & & & $1.239 \mathrm{E}+03$ & & $1.239 \mathrm{E}+03$ \\
\hline Pentachlorophenol & $5.866 E-03$ & $5.866 E-03$ & $5.866 E-03$ & & $5.199 E-06$ & $5.861 E-03$ & $5.861 E-03$ & $5.861 E-03$ & \\
\hline Hexachlorobenzene & $8.134 E-05$ & $4.186 E+00$ & $8.155 E-05$ & $4.186 E+00$ & $7.228 E-08$ & $8.147 E-05$ & $4.186 E+00$ & $8.168 E-05$ & $4.186 E+00$ \\
\hline Chlorobenzene & $3.833 E-06$ & $3.833 E-06$ & $3.833 E-06$ & & $3.397 E-09$ & $3.829 E-06$ & $3.829 E-06$ & $3.829 E-06$ & \\
\hline 2-Chloronaphthalene & $1.030 E-05$ & $1.030 E-05$ & $1.030 E-05$ & & $9.128 E-09$ & $1.029 E-05$ & $1.029 E-05$ & $1.029 E-05$ & \\
\hline Carbon Dioxide & $5.074 \mathrm{E}+03$ & $5.074 \mathrm{E}+03$ & $5.074 \mathrm{E}+03$ & & $4.498 \mathrm{E}+00$ & $5.070 \mathrm{E}+03$ & $5.070 \mathrm{E}+03$ & $5.070 \mathrm{E}+03$ & \\
\hline \multicolumn{10}{|l|}{ Cesium Acetate } \\
\hline Cesium Glycolate & $1.382 \mathrm{E}+01$ & $1.382 \mathrm{E}+01$ & $1.382 \mathrm{E}+01$ & & $1.225 \mathrm{E}-02$ & $1.380 \mathrm{E}+01$ & $1.380 \mathrm{E}+01$ & $1.380 \mathrm{E}+01$ & \\
\hline Dibenz[a,h]anthracene & $2.627 E-03$ & $2.627 E-03$ & $2.627 E-03$ & & $2.328 E-06$ & $2.625 E-03$ & $2.625 E-03$ & $2.625 E-03$ & \\
\hline 1,2-dibromoethane & $9.212 E-05$ & $9.212 E-05$ & $9.212 E-05$ & & $8.165 E-08$ & $9.204 E-05$ & $9.204 E-05$ & $9.204 E-05$ & \\
\hline Diethyl phthalate & $1.661 E-02$ & $1.661 E-02$ & $1.661 E-02$ & & $1.472 E-05$ & $1.659 E-02$ & $1.659 E-02$ & $1.659 E-02$ & \\
\hline \multicolumn{10}{|l|}{ Iron (III) Oxalate } \\
\hline Iron (III) Hydrogen EDTA & $3.827 \mathrm{E}+01$ & $3.827 \mathrm{E}+01$ & $3.827 \mathrm{E}+01$ & & 3.392E-02 & $3.824 \mathrm{E}+01$ & $3.824 \mathrm{E}+01$ & $3.824 \mathrm{E}+01$ & \\
\hline \multicolumn{10}{|l|}{ Iron (III) Hydroxide } \\
\hline \multicolumn{10}{|l|}{ Formic Acid, dimer } \\
\hline \multicolumn{10}{|l|}{ Hydrofluoride, dimer } \\
\hline 2,2'-Iminobisacetic acid & $7.322 \mathrm{E}+01$ & $7.322 \mathrm{E}+01$ & $7.322 \mathrm{E}+01$ & & $6.490 \mathrm{E}-02$ & $7.316 \mathrm{E}+01$ & $7.316 \mathrm{E}+01$ & $7.316 \mathrm{E}+01$ & \\
\hline Water & $9.000 \mathrm{E}+04$ & $9.010 \mathrm{E}+04$ & $9.010 \mathrm{E}+04$ & & $7.986 \mathrm{E}+01$ & $9.002 \mathrm{E}+04$ & $9.012 \mathrm{E}+04$ & $9.011 \mathrm{E}+04$ & \\
\hline \multicolumn{10}{|l|}{ Sulfuric Acid } \\
\hline Hydrogen Chloride & $7.715 \mathrm{E}+02$ & $7.715 \mathrm{E}+02$ & $7.715 \mathrm{E}+02$ & & $6.838 \mathrm{E}-01$ & $7.708 \mathrm{E}+02$ & $7.708 \mathrm{E}+02$ & $7.708 \mathrm{E}+02$ & \\
\hline Formic Acid & $2.561 \mathrm{E}+01$ & $2.561 \mathrm{E}+01$ & $2.561 \mathrm{E}+01$ & & $2.270 \mathrm{E}-02$ & $2.559 \mathrm{E}+01$ & $2.559 \mathrm{E}+01$ & $2.559 \mathrm{E}+01$ & \\
\hline Hydrofluoric Acid & $1.258 \mathrm{E}+02$ & $1.256 \mathrm{E}+02$ & $1.256 \mathrm{E}+02$ & & $1.113 \mathrm{E}-01$ & $1.255 \mathrm{E}+02$ & $1.254 \mathrm{E}+02$ & $1.254 \mathrm{E}+02$ & \\
\hline \multicolumn{10}{|l|}{ Mercury (II) Chloride } \\
\hline Mercury (elemental) & $8.810 \mathrm{E}-06$ & $8.810 \mathrm{E}-06$ & $8.810 \mathrm{E}-06$ & & 7.809E-09 & $8.802 \mathrm{E}-06$ & $8.802 \mathrm{E}-06$ & $8.802 \mathrm{E}-06$ & \\
\hline Mercury (II) Oxide & $7.944 \mathrm{E}+00$ & $4.933 \mathrm{E}+01$ & $7.939 \mathrm{E}+00$ & $4.139 \mathrm{E}+01$ & $7.037 \mathrm{E}-03$ & $7.932 \mathrm{E}+00$ & $4.932 \mathrm{E}+01$ & $7.927 \mathrm{E}+00$ & $4.139 \mathrm{E}+01$ \\
\hline Nitrous (III) Acid & $9.209 \mathrm{E}+03$ & $9.209 \mathrm{E}+03$ & $9.209 \mathrm{E}+03$ & & $8.162 \mathrm{E}+00$ & $9.200 \mathrm{E}+03$ & $9.200 \mathrm{E}+03$ & $9.200 \mathrm{E}+03$ & \\
\hline Nitric Acid & $4.247 \mathrm{E}+03$ & $4.247 \mathrm{E}+03$ & $4.247 \mathrm{E}+03$ & & $3.765 \mathrm{E}+00$ & $4.244 \mathrm{E}+03$ & $4.244 \mathrm{E}+03$ & $4.244 \mathrm{E}+03$ & \\
\hline Hexachlorobutadiene & $8.036 E-08$ & $8.036 E-08$ & $8.036 E-08$ & & $7.122 E-11$ & $8.028 E-08$ & $8.028 E-08$ & $8.028 E-08$ & \\
\hline Potassium Citrate & $1.826 \mathrm{E}+02$ & $1.826 \mathrm{E}+02$ & $1.826 \mathrm{E}+02$ & & $1.618 \mathrm{E}-01$ & $1.824 \mathrm{E}+02$ & $1.824 \mathrm{E}+02$ & $1.824 \mathrm{E}+02$ & \\
\hline \multicolumn{10}{|l|}{ Potassium Acetate } \\
\hline Potassium Chloride & $2.196 \mathrm{E}+03$ & $2.196 \mathrm{E}+03$ & $2.196 \mathrm{E}+03$ & & $1.947 \mathrm{E}+00$ & $2.194 \mathrm{E}+03$ & $2.194 \mathrm{E}+03$ & $2.194 \mathrm{E}+03$ & \\
\hline
\end{tabular}


WSRC-TR-2002-00550, Rev. 1 SRT-RPP-2002-0027.

\begin{tabular}{|c|c|c|c|c|c|c|c|c|c|}
\hline Stream & $\begin{array}{l}\text { SCW2 } \\
\text { Return }\end{array}$ & $\begin{array}{l}\text { SCW3 } \\
\text { Slurry }\end{array}$ & $\begin{array}{l}\text { SCW3 } \\
\text { Liquor }\end{array}$ & $\begin{array}{l}\text { SCW3 } \\
\text { Solids }\end{array}$ & $\begin{array}{c}\text { SCW3 } \\
\text { Recycle }\end{array}$ & $\begin{array}{l}\text { SCW3 } \\
\text { Return }\end{array}$ & $\begin{array}{l}\text { SCW4 } \\
\text { Slurry }\end{array}$ & $\begin{array}{l}\text { SCW4 } \\
\text { Liquor }\end{array}$ & $\begin{array}{l}\text { SCW4 } \\
\text { Solids }\end{array}$ \\
\hline Potassium Glycolate & $3.355 \mathrm{E}+02$ & $3.355 \mathrm{E}+02$ & $3.355 \mathrm{E}+02$ & & $2.973 \mathrm{E}-01$ & $3.352 \mathrm{E}+02$ & $3.352 \mathrm{E}+02$ & $3.352 \mathrm{E}+02$ & \\
\hline \multicolumn{10}{|l|}{ Potassium Nitrate (VI) } \\
\hline gamma-BHC (Lindane) & $2.244 E-04$ & $2.244 E-04$ & $2.244 E-04$ & & $1.989 E-07$ & $2.242 E-04$ & $2.242 E-04$ & $2.242 E-04$ & \\
\hline $\begin{array}{c}\text { 4-methyl-2-pentanone } \\
\text { (MIBK) }\end{array}$ & $4.274 E-05$ & $4.274 E-05$ & $4.274 E-05$ & & $3.788 E-08$ & $4.270 E-05$ & $4.270 E-05$ & $4.270 E-05$ & \\
\hline \multicolumn{10}{|l|}{ Magnesium Oxalate } \\
\hline Magnesium Dichromate (VI) & $4.619 \mathrm{E}-06$ & $4.630 \mathrm{E}-06$ & $4.630 \mathrm{E}-06$ & & $4.104 \mathrm{E}-09$ & $4.626 \mathrm{E}-06$ & $4.638 \mathrm{E}-06$ & $4.638 \mathrm{E}-06$ & \\
\hline Magnesium Hydroxide & & $2.392 \mathrm{E}+02$ & & $2.392 \mathrm{E}+02$ & & & $2.392 \mathrm{E}+02$ & & $2.392 \mathrm{E}+02$ \\
\hline Manganese (II) Oxalate & $1.710 \mathrm{E}-01$ & $1.710 \mathrm{E}-01$ & $1.710 \mathrm{E}-01$ & & $1.516 \mathrm{E}-04$ & $1.709 \mathrm{E}-01$ & $1.709 \mathrm{E}-01$ & $1.709 \mathrm{E}-01$ & \\
\hline \multicolumn{10}{|l|}{ Manganese (II) Hydroxide } \\
\hline Nitrogen & $8.806 \mathrm{E}-06$ & $8.806 \mathrm{E}-06$ & $8.806 \mathrm{E}-06$ & & $7.805 \mathrm{E}-09$ & $8.798 \mathrm{E}-06$ & $8.798 \mathrm{E}-06$ & $8.798 \mathrm{E}-06$ & \\
\hline Sodium Oxalate & & $1.196 \mathrm{E}+04$ & & $1.196 \mathrm{E}+04$ & & & $1.196 \mathrm{E}+04$ & & $1.196 \mathrm{E}+04$ \\
\hline Sodium Chromate (VI) & $4.350 \mathrm{E}+02$ & $4.349 \mathrm{E}+02$ & $4.349 \mathrm{E}+02$ & & $3.855 \mathrm{E}-01$ & $4.346 \mathrm{E}+02$ & $4.345 \mathrm{E}+02$ & $4.345 \mathrm{E}+02$ & \\
\hline Sodium Fluoride Sulfate & & $2.236 \mathrm{E}+04$ & & $2.236 \mathrm{E}+04$ & & & $2.235 \mathrm{E}+04$ & & $2.235 \mathrm{E}+04$ \\
\hline \multicolumn{10}{|l|}{ Sodium Acetate } \\
\hline \multicolumn{10}{|l|}{ Sodium Glycolate } \\
\hline \multicolumn{10}{|l|}{ Sodium Nitrite } \\
\hline Sodium Hydroxide & $3.555 \mathrm{E}+04$ & $3.555 \mathrm{E}+04$ & $3.555 \mathrm{E}+04$ & & $3.151 \mathrm{E}+01$ & $3.552 \mathrm{E}+04$ & $3.552 \mathrm{E}+04$ & $3.552 \mathrm{E}+04$ & \\
\hline Naphthalene & $1.133 E-05$ & $1.133 E-05$ & $1.133 E-05$ & & $1.004 E-08$ & $1.132 E-05$ & $1.132 E-05$ & $1.132 E-05$ & \\
\hline Sodium Aluminosilicate Gel & & $1.673 \mathrm{E}+03$ & & $1.673 \mathrm{E}+03$ & & & $1.676 \mathrm{E}+03$ & & $1.676 \mathrm{E}+03$ \\
\hline Nickel (II) Oxalate & $5.157 \mathrm{E}-01$ & $5.157 \mathrm{E}-01$ & $5.157 \mathrm{E}-01$ & & $4.571 \mathrm{E}-04$ & $5.152 \mathrm{E}-01$ & $5.152 \mathrm{E}-01$ & $5.152 \mathrm{E}-01$ & \\
\hline \multicolumn{10}{|l|}{ Nickel (II) Hydroxide } \\
\hline Oxygen & $4.900 \mathrm{E}-06$ & $4.900 \mathrm{E}-06$ & $4.900 \mathrm{E}-06$ & & 4.343E-09 & $4.895 \mathrm{E}-06$ & $4.895 \mathrm{E}-06$ & $4.895 \mathrm{E}-06$ & \\
\hline Phosphorus Pentoxide & $4.133 \mathrm{E}+01$ & $4.133 \mathrm{E}+01$ & $4.133 \mathrm{E}+01$ & & $3.663 \mathrm{E}-02$ & $4.129 \mathrm{E}+01$ & $4.129 \mathrm{E}+01$ & $4.129 \mathrm{E}+01$ & \\
\hline PYRENE & $3.535 E-04$ & $3.535 E-04$ & $3.535 E-04$ & & $3.133 E-07$ & $3.532 E-04$ & $3.531 E-04$ & $3.531 E-04$ & \\
\hline Silicon Dioxide & $6.531 \mathrm{E}+02$ & $6.521 \mathrm{E}+02$ & $6.521 \mathrm{E}+02$ & & $5.780 \mathrm{E}-01$ & $6.515 \mathrm{E}+02$ & $6.506 \mathrm{E}+02$ & $6.506 \mathrm{E}+02$ & \\
\hline Sulfur Trioxide & $4.848 \mathrm{E}+02$ & $4.876 \mathrm{E}+02$ & $4.876 \mathrm{E}+02$ & & $4.322 \mathrm{E}-01$ & $4.871 \mathrm{E}+02$ & $4.899 \mathrm{E}+02$ & $4.899 \mathrm{E}+02$ & \\
\hline 1,2,4-trichlorobenzene & $2.392 E-06$ & $2.392 E-06$ & $2.392 E-06$ & & $2.120 E-09$ & $2.390 E-06$ & $2.390 E-06$ & $2.390 E-06$ & \\
\hline 1,2,3-trichloropropane & $1.686 E-05$ & $1.686 E-05$ & $1.686 E-05$ & & $1.495 E-08$ & $1.685 E-05$ & $1.685 E-05$ & $1.685 E-05$ & \\
\hline TOLUENE & $1.486 E-06$ & $1.486 E-06$ & $1.485 E-06$ & & $1.317 E-09$ & $1.484 E-06$ & $1.484 E-06$ & $1.484 E-06$ & \\
\hline Zinc Dichloride Pentoxide & $3.159 \mathrm{E}+00$ & $3.066 \mathrm{E}+00$ & $3.066 \mathrm{E}+00$ & & $2.718 \mathrm{E}-03$ & $3.063 \mathrm{E}+00$ & $2.971 \mathrm{E}+00$ & $2.971 \mathrm{E}+00$ & \\
\hline Zinc Oxalate & $6.832 \mathrm{E}+01$ & $6.847 \mathrm{E}+01$ & $6.847 \mathrm{E}+01$ & & $6.069 \mathrm{E}-02$ & $6.841 \mathrm{E}+01$ & $6.857 \mathrm{E}+01$ & $6.857 \mathrm{E}+01$ & \\
\hline \multicolumn{10}{|l|}{ Zinc Hydroxide } \\
\hline Zirconium EDTA & $1.978 \mathrm{E}+00$ & $1.978 \mathrm{E}+00$ & $1.978 \mathrm{E}+00$ & & $1.753 \mathrm{E}-03$ & $1.976 \mathrm{E}+00$ & $1.976 \mathrm{E}+00$ & $1.976 \mathrm{E}+00$ & \\
\hline \multicolumn{10}{|l|}{ Zirconium Oxide } \\
\hline Total g/hr & $1.505 \mathrm{E}+05$ & $1.882 \mathrm{E}+05$ & $1.506 \mathrm{E}+05$ & $3.762 \mathrm{E}+04$ & $1.335 \mathrm{E}+02$ & $1.504 \mathrm{E}+05$ & $1.882 \mathrm{E}+05$ & $1.505 \mathrm{E}+05$ & $3.762 \mathrm{E}+04$ \\
\hline Volume, L/hr & $1.127 \mathrm{E}+02$ & $1.148 \mathrm{E}+02$ & $1.128 \mathrm{E}+02$ & $1.964 \mathrm{E}+00$ & $1.000 \mathrm{E}-01$ & $1.127 \mathrm{E}+02$ & $1.148 \mathrm{E}+02$ & $1.128 \mathrm{E}+02$ & $1.966 \mathrm{E}+00$ \\
\hline Enthalpy, cal/hr & $-4.793 \mathrm{E}+08$ & $-5.763 \mathrm{E}+08$ & $-4.796 \mathrm{E}+08$ & $-9.669 \mathrm{E}+07$ & $-4.251 \mathrm{E}+05$ & $-4.792 \mathrm{E}+08$ & $-5.763 \mathrm{E}+08$ & $-4.796 \mathrm{E}+08$ & $-9.668 \mathrm{E}+07$ \\
\hline \multicolumn{10}{|l|}{ Vapor fraction } \\
\hline Solid fraction & & $3.557 \mathrm{E}-02$ & & $1.000 \mathrm{E}+00$ & & & $3.557 \mathrm{E}-02$ & & $1.000 \mathrm{E}+00$ \\
\hline \multicolumn{10}{|l|}{ Organic fraction } \\
\hline Osmotic Pres, atm & $4.770 \mathrm{E}+02$ & $4.766 \mathrm{E}+02$ & $4.766 \mathrm{E}+02$ & & $4.766 \mathrm{E}+02$ & $4.766 \mathrm{E}+02$ & $4.761 \mathrm{E}+02$ & $4.761 \mathrm{E}+02$ & \\
\hline \multicolumn{10}{|l|}{ Redox Pot, volts } \\
\hline E-Con, 1/ohm-cm & $2.573 \mathrm{E}-01$ & $2.573 \mathrm{E}-01$ & $2.573 \mathrm{E}-01$ & & $2.573 \mathrm{E}-01$ & $2.573 \mathrm{E}-01$ & $2.573 \mathrm{E}-01$ & $2.573 \mathrm{E}-01$ & \\
\hline E-Con, cm2/ohm-mol & $1.822 \mathrm{E}+01$ & $1.823 \mathrm{E}+01$ & $1.823 \mathrm{E}+01$ & & $1.823 \mathrm{E}+01$ & $1.823 \mathrm{E}+01$ & $1.825 \mathrm{E}+01$ & $1.825 \mathrm{E}+01$ & \\
\hline Abs Visc, cP & $5.510 \mathrm{E}+00$ & $5.503 \mathrm{E}+00$ & $5.503 \mathrm{E}+00$ & & $5.503 \mathrm{E}+00$ & $5.503 \mathrm{E}+00$ & $5.496 \mathrm{E}+00$ & $5.496 \mathrm{E}+00$ & \\
\hline Rel Visc & $6.186 \mathrm{E}+00$ & $6.178 \mathrm{E}+00$ & $6.178 \mathrm{E}+00$ & & $6.178 \mathrm{E}+00$ & $6.178 \mathrm{E}+00$ & $6.171 \mathrm{E}+00$ & $6.171 \mathrm{E}+00$ & \\
\hline Ionic Strength & $1.000 \mathrm{E}+01$ & $9.992 \mathrm{E}+00$ & $9.992 \mathrm{E}+00$ & & $9.992 \mathrm{E}+00$ & $9.992 \mathrm{E}+00$ & $9.982 \mathrm{E}+00$ & $9.982 \mathrm{E}+00$ & \\
\hline
\end{tabular}


WSRC-TR-2002-00550, Rev. 1

SRT-RPP-2002-0027.

Table B- 2. Waste Feed Evaporator 5 Stage Model Results for UF 1.35 Density Case (cont'd) ${ }^{8}$

\begin{tabular}{|c|c|c|c|c|c|c|c|c|c|}
\hline Stream & $\begin{array}{c}\text { SCW4 } \\
\text { Recycle }\end{array}$ & $\begin{array}{l}\text { SCW4 } \\
\text { Return }\end{array}$ & $\begin{array}{l}\text { SCWash5 } \\
\text { Slurry }\end{array}$ & $\begin{array}{l}\text { SCW5 } \\
\text { Liquor }\end{array}$ & $\begin{array}{l}\text { SCW5 } \\
\text { Solids }\end{array}$ & $\begin{array}{c}\text { SCW5 } \\
\text { Recycle }\end{array}$ & $\begin{array}{l}\text { SCW5 } \\
\text { Return }\end{array}$ & Acid Rinse & Total Acid \\
\hline Phase & Aqueous & Aqueous & Mixed & Aqueous & Solid & Aqueous & Aqueous & Aqueous & Aqueous \\
\hline Temperature, $\mathrm{C}$ & 25 & 25 & 25 & 25 & 25 & 25 & 25 & 25 & 25 \\
\hline Pressure, atm & 1 & 1 & 1 & 1 & 1 & 1 & 1 & 1 & 1 \\
\hline $\mathrm{pH}$ & 15.1033 & 15.1033 & 15.1023 & 15.1023 & & 15.1023 & 15.1023 & -0.301785 & -0.301785 \\
\hline Total mol/hr & 5.63617 & 6353.22 & 6593.27 & 6358.79 & 234.475 & 5.63623 & 6353.16 & 53.9076 & 1633.01 \\
\hline Flow Units & $\mathrm{g} / \mathrm{hr}$ & $\mathrm{g} / \mathrm{hr}$ & $\mathrm{g} / \mathrm{hr}$ & $\mathrm{g} / \mathrm{hr}$ & $\mathrm{g} / \mathrm{hr}$ & $\mathrm{g} / \mathrm{hr}$ & $\mathrm{g} / \mathrm{hr}$ & $\mathrm{g} / \mathrm{hr}$ & $\mathrm{g} / \mathrm{hr}$ \\
\hline \multicolumn{10}{|l|}{ Acetic Acid, Dimer } \\
\hline Acetic Acid & $1.123 \mathrm{E}-02$ & $1.265 \mathrm{E}+01$ & $1.265 \mathrm{E}+01$ & $1.265 \mathrm{E}+01$ & & $1.122 \mathrm{E}-02$ & $1.264 \mathrm{E}+01$ & & \\
\hline Aluminum Oxide & $6.810 \mathrm{E}-01$ & $7.677 \mathrm{E}+02$ & $7.670 \mathrm{E}+02$ & $7.670 \mathrm{E}+02$ & & $6.798 \mathrm{E}-01$ & $7.663 \mathrm{E}+02$ & $1.214 \mathrm{E}+00$ & $3.678 \mathrm{E}+01$ \\
\hline Aldrin & $3.223 E-07$ & $3.633 E-04$ & $3.633 E-04$ & $3.633 E-04$ & & $3.221 E-07$ & $3.630 E-04$ & & \\
\hline Aluminum Hydrogen EDTA & $6.718 \mathrm{E}-02$ & $7.573 \mathrm{E}+01$ & $7.573 \mathrm{E}+01$ & $7.573 \mathrm{E}+01$ & & $6.712 \mathrm{E}-02$ & $7.566 \mathrm{E}+01$ & & \\
\hline \multicolumn{10}{|l|}{ Aluminum Hydroxide } \\
\hline Benzene & $1.582 E-09$ & $1.784 E-06$ & $1.784 E-06$ & $1.784 E-06$ & & $1.581 E-09$ & $1.782 E-06$ & & \\
\hline $\begin{array}{c}\text { Bis(2-ethylhexyl)phthalate } \\
\text { (BEHP) }\end{array}$ & $4.268 E-06$ & $4.811 E-03$ & $4.811 E-03$ & $4.811 E-03$ & & $4.264 E-06$ & $4.807 E-03$ & & \\
\hline Benzo(a)pyrene (BaP) & $3.033 E-08$ & $3.419 E-05$ & $4.181 E+00$ & $3.428 E-05$ & $4.181 E+00$ & $3.038 E-08$ & $3.424 E-05$ & & \\
\hline Phenol & $2.400 E-04$ & $2.705 E-01$ & $2.705 E-01$ & $2.705 E-01$ & & $2.398 E-04$ & $2.703 E-01$ & & \\
\hline \multicolumn{10}{|l|}{ Calcium Chloride Oxide } \\
\hline \multicolumn{10}{|l|}{ Calcium Oxalate } \\
\hline \multicolumn{10}{|l|}{ Calcium Oxalate Monohydrate } \\
\hline \multicolumn{10}{|l|}{ Calcium Carbonate } \\
\hline Calcium Dichromate (VI) & $7.348 \mathrm{E}-02$ & $8.282 \mathrm{E}+01$ & $8.285 \mathrm{E}+01$ & $8.285 \mathrm{E}+01$ & & $7.343 \mathrm{E}-02$ & $8.277 \mathrm{E}+01$ & & \\
\hline \multicolumn{10}{|l|}{ Calcium Citrate } \\
\hline Calcium Fluoride & & & $1.026 \mathrm{E}+02$ & & $1.026 \mathrm{E}+02$ & & & & \\
\hline Calcium Hydroxide & & & $1.238 \mathrm{E}+03$ & & $1.238 \mathrm{E}+03$ & & & & \\
\hline Pentachlorophenol & $5.195 E-06$ & $5.856 E-03$ & $5.856 E-03$ & $5.856 E-03$ & & $5.190 E-06$ & $5.851 E-03$ & & \\
\hline Hexachlorobenzene & $7.240 E-08$ & $8.161 E-05$ & $4.186 E+00$ & $8.182 E-05$ & $4.186 E+00$ & $7.252 E-08$ & $8.175 E-05$ & & \\
\hline Chlorobenzene & $3.394 E-09$ & $3.826 E-06$ & $3.826 E-06$ & $3.826 E-06$ & & $3.391 E-09$ & $3.823 E-06$ & & \\
\hline 2-Chloronaphthalene & $9.120 E-09$ & $1.028 E-05$ & $1.028 E-05$ & $1.028 E-05$ & & $9.112 E-09$ & $1.027 E-05$ & & \\
\hline Carbon Dioxide & $4.494 \mathrm{E}+00$ & $5.065 \mathrm{E}+03$ & $5.065 \mathrm{E}+03$ & $5.065 \mathrm{E}+03$ & & $4.490 \mathrm{E}+00$ & $5.061 \mathrm{E}+03$ & & \\
\hline \multicolumn{10}{|l|}{ Cesium Acetate } \\
\hline Cesium Glycolate & $1.224 \mathrm{E}-02$ & $1.379 \mathrm{E}+01$ & $1.379 \mathrm{E}+01$ & $1.379 \mathrm{E}+01$ & & $1.222 \mathrm{E}-02$ & $1.378 \mathrm{E}+01$ & & \\
\hline Dibenz[a,h]anthracene & $2.326 E-06$ & $2.622 E-03$ & $2.622 E-03$ & $2.622 E-03$ & & $2.324 E-06$ & $2.620 E-03$ & & \\
\hline 1,2-dibromoethane & $8.158 E-08$ & $9.196 E-05$ & $9.196 E-05$ & $9.196 E-05$ & & $8.151 E-08$ & $9.187 E-05$ & & \\
\hline Diethyl phthalate & $1.471 E-05$ & $1.658 E-02$ & $1.658 E-02$ & $1.658 E-02$ & & $1.469 E-05$ & $1.656 E-02$ & & \\
\hline \multicolumn{10}{|l|}{ Iron (III) Oxalate } \\
\hline Iron (III) Hydrogen EDTA & $3.389 \mathrm{E}-02$ & $3.820 \mathrm{E}+01$ & $3.820 \mathrm{E}+01$ & $3.820 \mathrm{E}+01$ & & $3.386 \mathrm{E}-02$ & $3.817 \mathrm{E}+01$ & & \\
\hline Iron (III) Hydroxide & & & & & & & & $9.175 \mathrm{E}-01$ & $2.779 \mathrm{E}+01$ \\
\hline \multicolumn{10}{|l|}{ Formic Acid, dimer } \\
\hline \multicolumn{10}{|l|}{ Hydrofluoride, dimer } \\
\hline 2,2'-Iminobisacetic acid & $6.484 \mathrm{E}-02$ & $7.309 \mathrm{E}+01$ & $7.309 \mathrm{E}+01$ & $7.309 \mathrm{E}+01$ & & $6.479 \mathrm{E}-02$ & $7.303 \mathrm{E}+01$ & & \\
\hline Water & $7.987 \mathrm{E}+01$ & $9.004 \mathrm{E}+04$ & $9.013 \mathrm{E}+04$ & $9.013 \mathrm{E}+04$ & & $7.989 \mathrm{E}+01$ & $9.005 \mathrm{E}+04$ & $9.343 \mathrm{E}+02$ & $2.830 \mathrm{E}+04$ \\
\hline \multicolumn{10}{|l|}{ Sulfuric Acid } \\
\hline Hydrogen Chloride & $6.832 \mathrm{E}-01$ & $7.701 \mathrm{E}+02$ & $7.701 \mathrm{E}+02$ & $7.701 \mathrm{E}+02$ & & $6.826 \mathrm{E}-01$ & $7.695 \mathrm{E}+02$ & & \\
\hline Formic Acid & $2.268 \mathrm{E}-02$ & $2.556 \mathrm{E}+01$ & $2.556 \mathrm{E}+01$ & $2.556 \mathrm{E}+01$ & & $2.266 \mathrm{E}-02$ & $2.554 \mathrm{E}+01$ & & \\
\hline Hydrofluoric Acid & $1.111 \mathrm{E}-01$ & $1.253 \mathrm{E}+02$ & $1.251 \mathrm{E}+02$ & $1.251 \mathrm{E}+02$ & & $1.109 \mathrm{E}-01$ & $1.250 \mathrm{E}+02$ & & \\
\hline \multicolumn{10}{|l|}{ Mercury (II) Chloride } \\
\hline Mercury (elemental) & $7.802 \mathrm{E}-09$ & 8.794E-06 & $8.794 \mathrm{E}-06$ & $8.794 \mathrm{E}-06$ & & $7.795 \mathrm{E}-09$ & 8.787E-06 & & \\
\hline Mercury (II) Oxide & $7.026 \mathrm{E}-03$ & $7.920 \mathrm{E}+00$ & $4.931 \mathrm{E}+01$ & $7.915 \mathrm{E}+00$ & $4.140 \mathrm{E}+01$ & $7.016 \mathrm{E}-03$ & $7.908 \mathrm{E}+00$ & & \\
\hline Nitrous (III) Acid & $8.155 \mathrm{E}+00$ & $9.192 \mathrm{E}+03$ & $9.192 \mathrm{E}+03$ & $9.192 \mathrm{E}+03$ & & $8.148 \mathrm{E}+00$ & $9.184 \mathrm{E}+03$ & & \\
\hline Nitric Acid & $3.761 \mathrm{E}+00$ & $4.240 \mathrm{E}+03$ & $4.240 \mathrm{E}+03$ & $4.240 \mathrm{E}+03$ & & $3.758 \mathrm{E}+00$ & $4.236 \mathrm{E}+03$ & $1.266 \mathrm{E}+02$ & $3.834 \mathrm{E}+03$ \\
\hline Hexachlorobutadiene & $7.116 E-11$ & $8.021 E-08$ & $8.021 E-08$ & $8.021 E-08$ & & $7.110 E-11$ & $8.014 E-08$ & & \\
\hline Potassium Citrate & $1.617 \mathrm{E}-01$ & $1.822 \mathrm{E}+02$ & $1.822 \mathrm{E}+02$ & $1.822 \mathrm{E}+02$ & & $1.615 \mathrm{E}-01$ & $1.821 \mathrm{E}+02$ & & \\
\hline \multicolumn{10}{|l|}{ Potassium Acetate } \\
\hline Potassium Chloride & $1.945 \mathrm{E}+00$ & $2.192 \mathrm{E}+03$ & $2.192 \mathrm{E}+03$ & $2.192 \mathrm{E}+03$ & & $1.943 \mathrm{E}+00$ & $2.190 \mathrm{E}+03$ & & \\
\hline
\end{tabular}


WSRC-TR-2002-00550, Rev. 1

SRT-RPP-2002-0027.

\begin{tabular}{|c|c|c|c|c|c|c|c|c|c|}
\hline Stream & $\begin{array}{l}\text { SCW4 } \\
\text { Recycle }\end{array}$ & $\begin{array}{l}\text { SCW4 } \\
\text { Return }\end{array}$ & $\begin{array}{l}\text { SCWash5 } \\
\text { Slurry }\end{array}$ & $\begin{array}{l}\text { SCW5 } \\
\text { Liquor }\end{array}$ & $\begin{array}{l}\text { SCW5 } \\
\text { Solids }\end{array}$ & $\begin{array}{l}\text { SCW5 } \\
\text { Recycle }\end{array}$ & $\begin{array}{l}\text { SCW5 } \\
\text { Return }\end{array}$ & Acid Rinse & Total Acid \\
\hline Potassium Glycolate & $2.971 \mathrm{E}-01$ & $3.349 \mathrm{E}+02$ & $3.349 \mathrm{E}+02$ & $3.349 \mathrm{E}+02$ & & $2.968 \mathrm{E}-01$ & $3.346 \mathrm{E}+02$ & & \\
\hline \multicolumn{10}{|l|}{ Potassium Nitrate (VI) } \\
\hline gamma-BHC (Lindane) & $1.987 E-07$ & $2.240 E-04$ & $2.240 E-04$ & $2.240 E-04$ & & $1.985 E-07$ & $2.238 E-04$ & & \\
\hline $\begin{array}{l}\text { 4-methyl-2-pentanone } \\
\text { (MIBK) }\end{array}$ & $3.785 E-08$ & $4.267 E-05$ & $4.267 E-05$ & $4.267 E-05$ & & $3.782 E-08$ & $4.263 E-05$ & & \\
\hline \multicolumn{10}{|l|}{ Magnesium Oxalate } \\
\hline Magnesium Dichromate (VI) & 4.111E-09 & $4.633 \mathrm{E}-06$ & $4.645 \mathrm{E}-06$ & $4.645 \mathrm{E}-06$ & & 4.117E-09 & $4.641 \mathrm{E}-06$ & & \\
\hline Magnesium Hydroxide & & & $2.392 \mathrm{E}+02$ & & $2.392 \mathrm{E}+02$ & & & 4.297E-02 & $1.302 \mathrm{E}+00$ \\
\hline Manganese (II) Oxalate & $1.515 \mathrm{E}-04$ & $1.707 \mathrm{E}-01$ & $1.707 \mathrm{E}-01$ & $1.707 \mathrm{E}-01$ & & $1.513 \mathrm{E}-04$ & $1.706 \mathrm{E}-01$ & & \\
\hline Manganese (II) Hydroxide & & & & & & & & $5.433 \mathrm{E}-02$ & $1.646 \mathrm{E}+00$ \\
\hline Nitrogen & $7.798 \mathrm{E}-09$ & $8.791 \mathrm{E}-06$ & $8.791 \mathrm{E}-06$ & 8.791E-06 & & 7.792E-09 & $8.783 \mathrm{E}-06$ & & \\
\hline Sodium Oxalate & & & $1.196 \mathrm{E}+04$ & & $1.196 \mathrm{E}+04$ & & & & \\
\hline Sodium Chromate (VI) & $3.851 \mathrm{E}-01$ & $4.341 \mathrm{E}+02$ & $4.341 \mathrm{E}+02$ & $4.341 \mathrm{E}+02$ & & $3.848 \mathrm{E}-01$ & $4.337 \mathrm{E}+02$ & & \\
\hline Sodium Fluoride Sulfate & & & $2.235 \mathrm{E}+04$ & & $2.235 \mathrm{E}+04$ & & & & \\
\hline \multicolumn{10}{|l|}{ Sodium Acetate } \\
\hline \multicolumn{10}{|l|}{ Sodium Glycolate } \\
\hline \multicolumn{10}{|l|}{ Sodium Nitrite } \\
\hline Sodium Hydroxide & $3.148 \mathrm{E}+01$ & $3.549 \mathrm{E}+04$ & $3.549 \mathrm{E}+04$ & $3.549 \mathrm{E}+04$ & & $3.146 \mathrm{E}+01$ & $3.546 \mathrm{E}+04$ & $4.629 \mathrm{E}-01$ & $1.402 \mathrm{E}+01$ \\
\hline Naphthalene & $1.003 E-08$ & $1.131 E-05$ & $1.131 E-05$ & $1.131 E-05$ & & $1.003 E-08$ & $1.130 E-05$ & & \\
\hline Sodium Aluminosilicate Gel & & & $1.678 \mathrm{E}+03$ & & $1.678 \mathrm{E}+03$ & & & & \\
\hline Nickel (II) Oxalate & $4.567 \mathrm{E}-04$ & $5.148 \mathrm{E}-01$ & $5.148 \mathrm{E}-01$ & $5.148 \mathrm{E}-01$ & & $4.563 \mathrm{E}-04$ & $5.143 \mathrm{E}-01$ & & \\
\hline Nickel (II) Hydroxide & & & & & & & & $1.663 \mathrm{E}-01$ & $5.039 \mathrm{E}+00$ \\
\hline Oxygen & 4.339E-09 & $4.891 \mathrm{E}-06$ & $4.891 \mathrm{E}-06$ & $4.891 \mathrm{E}-06$ & & $4.335 \mathrm{E}-09$ & $4.887 \mathrm{E}-06$ & & \\
\hline Phosphorus Pentoxide & $3.660 \mathrm{E}-02$ & $4.126 \mathrm{E}+01$ & $4.126 \mathrm{E}+01$ & $4.126 \mathrm{E}+01$ & & $3.657 \mathrm{E}-02$ & $4.122 \mathrm{E}+01$ & & \\
\hline PYRENE & $3.130 E-07$ & $3.528 E-04$ & $3.528 E-04$ & $3.528 E-04$ & & $3.127 E-07$ & $3.525 E-04$ & & \\
\hline Silicon Dioxide & $5.767 \mathrm{E}-01$ & $6.500 \mathrm{E}+02$ & $6.491 \mathrm{E}+02$ & $6.491 \mathrm{E}+02$ & & $5.753 \mathrm{E}-01$ & $6.485 \mathrm{E}+02$ & $1.093 \mathrm{E}-01$ & $3.311 \mathrm{E}+00$ \\
\hline Sulfur Trioxide & $4.342 \mathrm{E}-01$ & $4.895 \mathrm{E}+02$ & $4.923 \mathrm{E}+02$ & $4.923 \mathrm{E}+02$ & & $4.363 \mathrm{E}-01$ & $4.918 \mathrm{E}+02$ & & \\
\hline 1,2,4-trichlorobenzene & $2.119 E-09$ & $2.388 E-06$ & $2.388 E-06$ & $2.388 E-06$ & & $2.117 E-09$ & $2.386 E-06$ & & \\
\hline 1,2,3-trichloropropane & $1.493 E-08$ & $1.683 E-05$ & $1.683 E-05$ & $1.683 E-05$ & & $1.492 E-08$ & $1.682 E-05$ & & \\
\hline TOLUENE & $1.316 E-09$ & $1.483 E-06$ & $1.483 E-06$ & $1.483 E-06$ & & $1.314 E-09$ & $1.482 E-06$ & & \\
\hline Zinc Dichloride Pentoxide & $2.633 \mathrm{E}-03$ & $2.968 \mathrm{E}+00$ & $2.876 \mathrm{E}+00$ & $2.876 \mathrm{E}+00$ & & $2.549 \mathrm{E}-03$ & $2.873 \mathrm{E}+00$ & & \\
\hline Zinc Oxalate & $6.077 \mathrm{E}-02$ & $6.851 \mathrm{E}+01$ & $6.866 \mathrm{E}+01$ & $6.866 \mathrm{E}+01$ & & $6.086 \mathrm{E}-02$ & $6.860 \mathrm{E}+01$ & & \\
\hline \multicolumn{10}{|l|}{ Zinc Hydroxide } \\
\hline Zirconium EDTA & $1.751 \mathrm{E}-03$ & $1.974 \mathrm{E}+00$ & $1.974 \mathrm{E}+00$ & $1.974 \mathrm{E}+00$ & & $1.750 \mathrm{E}-03$ & $1.972 \mathrm{E}+00$ & & \\
\hline Zirconium Oxide & & & & & & & & $3.279 \mathrm{E}-01$ & $9.932 \mathrm{E}+00$ \\
\hline Total $\mathrm{g} / \mathrm{hr}$ & $1.334 \mathrm{E}+02$ & $1.504 \mathrm{E}+05$ & $1.881 \mathrm{E}+05$ & $1.505 \mathrm{E}+05$ & $3.761 \mathrm{E}+04$ & $1.334 \mathrm{E}+02$ & $1.504 \mathrm{E}+05$ & $1.064 \mathrm{E}+03$ & $3.224 \mathrm{E}+04$ \\
\hline Volume, L/hr & $1.000 \mathrm{E}-01$ & $1.127 \mathrm{E}+02$ & $1.148 \mathrm{E}+02$ & $1.128 \mathrm{E}+02$ & $1.968 \mathrm{E}+00$ & $1.000 \mathrm{E}-01$ & $1.127 \mathrm{E}+02$ & $1.000 \mathrm{E}+00$ & $3.029 \mathrm{E}+01$ \\
\hline Enthalpy, cal/hr & $-4.251 \mathrm{E}+05$ & $-4.792 \mathrm{E}+08$ & $-5.762 \mathrm{E}+08$ & $-4.796 \mathrm{E}+08$ & $-9.668 \mathrm{E}+07$ & $-4.251 \mathrm{E}+05$ & $-4.791 \mathrm{E}+08$ & $-3.648 \mathrm{E}+06$ & $-1.105 \mathrm{E}+08$ \\
\hline \multicolumn{10}{|l|}{ Vapor fraction } \\
\hline Solid fraction & & & $3.556 \mathrm{E}-02$ & & $1.000 \mathrm{E}+00$ & & & & \\
\hline \multicolumn{10}{|l|}{ Organic fraction } \\
\hline Osmotic Pres, atm & $4.761 \mathrm{E}+02$ & $4.761 \mathrm{E}+02$ & $4.756 \mathrm{E}+02$ & $4.756 \mathrm{E}+02$ & & $4.756 \mathrm{E}+02$ & $4.756 \mathrm{E}+02$ & $9.744 \mathrm{E}+01$ & $9.744 \mathrm{E}+01$ \\
\hline \multicolumn{10}{|l|}{ Redox Pot, volts } \\
\hline E-Con, 1/ohm-cm & $2.573 \mathrm{E}-01$ & $2.573 \mathrm{E}-01$ & $2.573 \mathrm{E}-01$ & $2.573 \mathrm{E}-01$ & & $2.573 \mathrm{E}-01$ & $2.573 \mathrm{E}-01$ & $5.395 \mathrm{E}-01$ & $5.395 \mathrm{E}-01$ \\
\hline E-Con, cm2/ohm-mol & $1.825 \mathrm{E}+01$ & $1.825 \mathrm{E}+01$ & $1.826 \mathrm{E}+01$ & $1.826 \mathrm{E}+01$ & & $1.826 \mathrm{E}+01$ & $1.826 \mathrm{E}+01$ & $2.691 \mathrm{E}+02$ & $2.691 \mathrm{E}+02$ \\
\hline Abs Visc, cP & $5.496 \mathrm{E}+00$ & $5.496 \mathrm{E}+00$ & $5.490 \mathrm{E}+00$ & $5.490 \mathrm{E}+00$ & & $5.490 \mathrm{E}+00$ & $5.490 \mathrm{E}+00$ & 9.672E-01 & 9.672E-01 \\
\hline Rel Visc & $6.171 \mathrm{E}+00$ & $6.171 \mathrm{E}+00$ & $6.163 \mathrm{E}+00$ & $6.163 \mathrm{E}+00$ & & $6.163 \mathrm{E}+00$ & $6.163 \mathrm{E}+00$ & $1.086 \mathrm{E}+00$ & $1.086 \mathrm{E}+00$ \\
\hline Ionic Strength & $9.982 \mathrm{E}+00$ & $9.982 \mathrm{E}+00$ & $9.973 \mathrm{E}+00$ & $9.973 \mathrm{E}+00$ & & $9.973 \mathrm{E}+00$ & $9.973 \mathrm{E}+00$ & $2.114 \mathrm{E}+00$ & $2.114 \mathrm{E}+00$ \\
\hline
\end{tabular}


WSRC-TR-2002-00550, Rev. 1

SRT-RPP-2002-0027.

Table B- 2. Waste Feed Evaporator 5 Stage Model Results for UF 1.35 Density Case (cont'd) ${ }^{8}$

\begin{tabular}{|c|c|c|c|c|c|c|}
\hline Stream & $\begin{array}{l}\text { Caustic } \\
\text { Rinse }\end{array}$ & $\begin{array}{l}\text { Total C } \\
\text { Rinse }\end{array}$ & $\begin{array}{c}\text { Acc1 } \\
\text { Recycle }\end{array}$ & $\begin{array}{c}\text { Total } \\
\text { Recycle }\end{array}$ & $\begin{array}{l}\text { HLW OG } \\
\text { Cond }\end{array}$ & Evap Feed \\
\hline Phase & Aqueous & Aqueous & Mixed & Mixed & Mixed & Mixed \\
\hline Temperature, $\mathrm{C}$ & 25 & 25 & 25 & 25 & 25 & 25 \\
\hline Pressure, atm & 1 & 1 & 1 & 1 & 1 & 1 \\
\hline $\mathrm{pH}$ & 12.8948 & 12.8948 & 14.6137 & 12.8607 & 11.2694 & 13.0705 \\
\hline Total $\mathrm{mol} / \mathrm{hr}$ & 55.498 & 5043.55 & 38.4571 & 7912.79 & 188132 & 197463 \\
\hline Flow Units & $\mathrm{g} / \mathrm{hr}$ & $\mathrm{g} / \mathrm{hr}$ & $\mathrm{g} / \mathrm{hr}$ & $\mathrm{g} / \mathrm{hr}$ & $\mathrm{g} / \mathrm{hr}$ & $\mathrm{g} / \mathrm{hr}$ \\
\hline \multirow{2}{*}{\multicolumn{7}{|c|}{$\begin{array}{c}\text { Acetic Acid, Dimer } \\
\text { Acetic Acid }\end{array}$}} \\
\hline & & & & & & \\
\hline Aluminum Oxide & & & $2.910 \mathrm{E}+00$ & $6.382 \mathrm{E}+01$ & $1.231 \mathrm{E}+00$ & $2.657 \mathrm{E}+03$ \\
\hline Aldrin & & & $2.806 E-06$ & $7.204 E-05$ & & $4.195 E+00$ \\
\hline Aluminum Hydrogen EDTA & & & $5.848 \mathrm{E}-01$ & $1.727 \mathrm{E}+01$ & & $1.741 \mathrm{E}+03$ \\
\hline Aluminum Hydroxide & & & & $4.776 \mathrm{E}+01$ & & $3.103 \mathrm{E}+03$ \\
\hline Benzene & & & $1.377 E-08$ & $3.537 E-07$ & & $4.194 E+00$ \\
\hline $\begin{array}{c}\text { Bis(2-ethylhexyl)phthalate } \\
\text { (BEHP) }\end{array}$ & & & $3.715 E-05$ & $9.539 E-04$ & & $4.193 E+00$ \\
\hline Benzo(a)pyrene (BaP) & & & $2.967 E-07$ & $6.746 E-06$ & & $4.181 E+00$ \\
\hline Phenol & & & $2.089 E-03$ & $5.364 E-02$ & & $4.196 E+00$ \\
\hline Calcium Chloride Oxide & & & & & $5.464 \mathrm{E}+02$ & \\
\hline Calcium Oxalate & & & $3.105 \mathrm{E}-01$ & & $5.512 \mathrm{E}+00$ & $6.736 \mathrm{E}+02$ \\
\hline Calcium Oxalate Monohydrate & & & & & $2.059 \mathrm{E}+00$ & \\
\hline Calcium Carbonate & & & & $5.280 \mathrm{E}+00$ & & \\
\hline \multicolumn{7}{|l|}{ Calcium Dichromate (VI) } \\
\hline Calcium Citrate & & & & $1.867 \mathrm{E}+00$ & & \\
\hline Calcium Fluoride & & & & & $5.358 \mathrm{E}+02$ & $3.237 \mathrm{E}+02$ \\
\hline \multicolumn{7}{|l|}{ Calcium Hydroxide } \\
\hline Pentachlorophenol & & & $4.522 E-05$ & $1.161 E-03$ & & $4.196 E+00$ \\
\hline Hexachlorobenzene & & & $7.082 E-07$ & $1.610 E-05$ & & $4.188 E+00$ \\
\hline Chlorobenzene & & & $2.954 E-08$ & $7.586 E-07$ & & $4.190 E+00$ \\
\hline 2-Chloronaphthalene & & & $7.938 E-08$ & $2.038 E-06$ & & $4.194 E+00$ \\
\hline Carbon Dioxide & & & $3.911 \mathrm{E}+01$ & $1.002 \mathrm{E}+03$ & & $7.847 \mathrm{E}+04$ \\
\hline Cesium Acetate & & & $9.831 \mathrm{E}-02$ & $2.524 \mathrm{E}+00$ & & $1.972 \mathrm{E}+02$ \\
\hline \multicolumn{7}{|l|}{ Cesium Glycolate } \\
\hline Dibenz[a,h]anthracene & & & $2.025 E-05$ & $5.199 E-04$ & & $4.191 E+00$ \\
\hline 1,2-dibromoethane & & & $7.101 E-07$ & $1.823 E-05$ & & $2.806 E+01$ \\
\hline Diethyl phthalate & & & $1.280 E-04$ & $3.287 E-03$ & & $4.202 E+00$ \\
\hline Iron (III) Oxalate & & & & & $1.343 \mathrm{E}+00$ & \\
\hline Iron (III) Hydrogen EDTA & & & $2.950 \mathrm{E}-01$ & $4.792 \mathrm{E}+00$ & & \\
\hline Iron (III) Hydroxide & & & & $2.866 \mathrm{E}+01$ & $1.547 \mathrm{E}+02$ & \\
\hline \multicolumn{7}{|l|}{ Formic Acid, dimer } \\
\hline \multicolumn{7}{|l|}{ Hydrofluoride, dimer } \\
\hline 2,2'-Iminobisacetic acid & & & $5.644 \mathrm{E}-01$ & $1.449 \mathrm{E}+01$ & & $1.132 \mathrm{E}+03$ \\
\hline Water & $9.980 \mathrm{E}+02$ & $9.070 \mathrm{E}+04$ & $5.357 \mathrm{E}+02$ & $1.371 \mathrm{E}+05$ & $3.361 \mathrm{E}+06$ & $3.290 \mathrm{E}+06$ \\
\hline \multicolumn{7}{|l|}{ Sulfuric Acid } \\
\hline Hydrogen Chloride & & & $5.124 \mathrm{E}+00$ & $1.301 \mathrm{E}+02$ & $1.862 \mathrm{E}+04$ & \\
\hline Formic Acid & & & $1.974 \mathrm{E}-01$ & $5.069 \mathrm{E}+00$ & & $3.961 \mathrm{E}+02$ \\
\hline Hydrofluoric Acid & & & $5.609 \mathrm{E}-01$ & $2.471 \mathrm{E}+01$ & $2.116 \mathrm{E}+01$ & $3.388 \mathrm{E}+03$ \\
\hline \multicolumn{7}{|l|}{ Mercury (II) Chloride } \\
\hline Mercury (elemental) & & & $6.791 \mathrm{E}-08$ & $1.744 \mathrm{E}-06$ & & \\
\hline Mercury (II) Oxide & & & $2.148 \mathrm{E}-02$ & $1.534 \mathrm{E}+00$ & & $1.361 \mathrm{E}+02$ \\
\hline Nitrous (III) Acid & & & $6.437 \mathrm{E}+01$ & $4.828 \mathrm{E}+02$ & $3.708 \mathrm{E}+03$ & $9.342 \mathrm{E}+04$ \\
\hline Nitric Acid & & & $3.274 \mathrm{E}+01$ & $4.675 \mathrm{E}+03$ & $1.888 \mathrm{E}+03$ & $4.525 \mathrm{E}+04$ \\
\hline Hexachlorobutadiene & & & $6.194 E-10$ & $1.590 E-08$ & & $4.200 E+00$ \\
\hline Potassium Citrate & & & $1.407 \mathrm{E}+00$ & $3.384 \mathrm{E}+01$ & & $2.823 \mathrm{E}+03$ \\
\hline Potassium Acetate & & & & & & $2.195 \mathrm{E}+02$ \\
\hline Potassium Chloride & & & $1.862 \mathrm{E}+01$ & $4.797 \mathrm{E}+02$ & $1.845 \mathrm{E}+04$ & $1.350 \mathrm{E}+03$ \\
\hline
\end{tabular}


WSRC-TR-2002-00550, Rev. 1 SRT-RPP-2002-0027.

\begin{tabular}{|c|c|c|c|c|c|c|}
\hline Stream & $\begin{array}{c}\text { Caustic } \\
\text { Rinse }\end{array}$ & $\begin{array}{c}\text { Total C } \\
\text { Rinse }\end{array}$ & $\begin{array}{c}\text { Acc1 } \\
\text { Recycle }\end{array}$ & $\begin{array}{c}\text { Total } \\
\text { Recycle }\end{array}$ & $\begin{array}{l}\text { HLW OG } \\
\text { Cond }\end{array}$ & Evap Feed \\
\hline \multicolumn{7}{|l|}{ Potassium Glycolate } \\
\hline Potassium Nitrate (VI) & & & & & & $2.357 \mathrm{E}+04$ \\
\hline gamma-BHC (Lindane) & & & $1.730 E-06$ & $4.442 E-05$ & & $4.209 E+00$ \\
\hline $\begin{array}{c}\text { 4-methyl-2-pentanone } \\
\text { (MIBK) }\end{array}$ & & & $3.295 E-07$ & $8.460 E-06$ & & $4.197 E+00$ \\
\hline Magnesium Oxalate & & & $1.056 \mathrm{E}-07$ & $1.488 \mathrm{E}-03$ & $2.060 \mathrm{E}+00$ & \\
\hline \multicolumn{7}{|l|}{ Magnesium Dichromate (VI) } \\
\hline Magnesium Hydroxide & & & $3.581 \mathrm{E}-07$ & $1.301 \mathrm{E}+00$ & $2.368 \mathrm{E}+02$ & \\
\hline Manganese (II) Oxalate & & & $1.318 \mathrm{E}-03$ & $2.485 \mathrm{E}-01$ & & \\
\hline Manganese (II) Hydroxide & & & & $1.512 \mathrm{E}+00$ & & \\
\hline Nitrogen & & & $6.788 \mathrm{E}-08$ & $1.743 \mathrm{E}-06$ & & \\
\hline Sodium Oxalate & & & $5.275 \mathrm{E}-01$ & & & $1.433 \mathrm{E}+04$ \\
\hline Sodium Chromate $(\mathrm{VI})$ & & & $4.162 \mathrm{E}+00$ & $1.069 \mathrm{E}+02$ & & $8.349 \mathrm{E}+03$ \\
\hline Sodium Fluoride Sulfate & & & $1.631 \mathrm{E}+00$ & & & \\
\hline Sodium Acetate & & & $9.147 \mathrm{E}-02$ & $2.349 \mathrm{E}+00$ & & \\
\hline Sodium Glycolate & & & $2.271 \mathrm{E}+00$ & $5.832 \mathrm{E}+01$ & & $4.556 \mathrm{E}+03$ \\
\hline Sodium Nitrite & & & $9.697 \mathrm{E}+00$ & $1.966 \mathrm{E}+03$ & $3.054 \mathrm{E}+03$ & $6.342 \mathrm{E}+04$ \\
\hline Sodium Hydroxide & $4.000 \mathrm{E}+00$ & $3.635 \mathrm{E}+02$ & $2.023 \mathrm{E}+02$ & $6.114 \mathrm{E}+03$ & $2.572 \mathrm{E}+04$ & $3.253 \mathrm{E}+05$ \\
\hline Naphthalene & & & $8.735 E-08$ & $2.243 E-06$ & & $4.188 E+00$ \\
\hline Sodium Aluminosilicate Gel & & & $9.582 \mathrm{E}-01$ & $3.466 \mathrm{E}+02$ & $2.920 \mathrm{E}+03$ & \\
\hline Nickel (II) Oxalate & & & $3.975 \mathrm{E}-03$ & $8.077 \mathrm{E}+00$ & & \\
\hline \multicolumn{7}{|l|}{ Nickel (II) Hydroxide } \\
\hline Oxygen & & & $3.777 \mathrm{E}-08$ & $9.698 \mathrm{E}-07$ & & \\
\hline Phosphorus Pentoxide & & & $3.186 \mathrm{E}-01$ & $8.180 \mathrm{E}+00$ & & $6.391 \mathrm{E}+02$ \\
\hline PYRENE & & & $2.725 E-06$ & $6.996 E-05$ & & $4.202 E+00$ \\
\hline Silicon Dioxide & & & $8.693 \mathrm{E}-01$ & $3.496 \mathrm{E}+00$ & $3.339 \mathrm{E}+02$ & \\
\hline Sulfur Trioxide & & & $1.550 \mathrm{E}+01$ & $1.074 \mathrm{E}+02$ & $5.781 \mathrm{E}+02$ & $4.702 \mathrm{E}+04$ \\
\hline 1,2,4-trichlorobenzene & & & $1.844 E-08$ & $4.735 E-07$ & & $4.193 E+00$ \\
\hline 1,2,3-trichloropropane & & & $1.300 E-07$ & $3.338 E-06$ & & $4.198 E+00$ \\
\hline TOLUENE & & & $1.145 E-08$ & $2.940 E-07$ & & $4.194 E+00$ \\
\hline Zinc Dichloride Pentoxide & & & & $5.119 \mathrm{E}+00$ & $9.198 \mathrm{E}+00$ & \\
\hline Zinc Oxalate & & & $5.678 \mathrm{E}-01$ & $5.908 \mathrm{E}+00$ & & \\
\hline Zinc Hydroxide & & & & & $7.281 \mathrm{E}+02$ & \\
\hline Zirconium EDTA & & & $1.524 \mathrm{E}-02$ & $7.440 \mathrm{E}-01$ & & \\
\hline Zirconium Oxide & & & & $9.818 \mathrm{E}+00$ & & \\
\hline Total $\mathrm{g} / \mathrm{hr}$ & $1.002 \mathrm{E}+03$ & $9.106 \mathrm{E}+04$ & $9.416 \mathrm{E}+02$ & $1.528 \mathrm{E}+05$ & $3.438 \mathrm{E}+06$ & $4.012 \mathrm{E}+06$ \\
\hline Volume, L/hr & $1.001 \mathrm{E}+00$ & $9.095 \mathrm{E}+01$ & 7.001E-01 & $1.436 \mathrm{E}+02$ & $3.405 \mathrm{E}+03$ & $3.551 \mathrm{E}+03$ \\
\hline Enthalpy, cal/hr & $-3.796 \mathrm{E}+06$ & $-3.449 \mathrm{E}+08$ & $-2.914 \mathrm{E}+06$ & $-5.506 \mathrm{E}+08$ & $-1.289 \mathrm{E}+10$ & $-1.404 \mathrm{E}+10$ \\
\hline \multicolumn{7}{|l|}{ Vapor fraction } \\
\hline Solid fraction & & & $2.980 \mathrm{E}-04$ & $1.707 \mathrm{E}-04$ & $1.181 \mathrm{E}-04$ & $2.238 \mathrm{E}-04$ \\
\hline \multicolumn{7}{|l|}{ Organic fraction } \\
\hline Osmotic Pres, atm & $4.622 \mathrm{E}+00$ & $4.622 \mathrm{E}+00$ & $3.275 \mathrm{E}+02$ & $4.822 \mathrm{E}+01$ & $1.256 \mathrm{E}+01$ & $9.071 \mathrm{E}+01$ \\
\hline \multicolumn{7}{|l|}{ Redox Pot, volts } \\
\hline E-Con, 1/ohm-cm & $2.233 \mathrm{E}-02$ & $2.233 \mathrm{E}-02$ & $2.600 \mathrm{E}-01$ & $9.183 \mathrm{E}-02$ & $2.639 \mathrm{E}-02$ & $1.378 \mathrm{E}-01$ \\
\hline E-Con, cm2/ohm-mol & $2.235 \mathrm{E}+02$ & $2.235 \mathrm{E}+02$ & $2.138 \mathrm{E}+01$ & $3.893 \mathrm{E}+01$ & $9.004 \mathrm{E}+01$ & $6.830 \mathrm{E}+01$ \\
\hline Abs Visc, cP & $9.101 \mathrm{E}-01$ & $9.101 \mathrm{E}-01$ & $4.450 \mathrm{E}+00$ & $1.063 \mathrm{E}+00$ & $9.062 \mathrm{E}-01$ & $1.494 \mathrm{E}+00$ \\
\hline Rel Visc & $1.022 \mathrm{E}+00$ & $1.022 \mathrm{E}+00$ & $4.996 \mathrm{E}+00$ & $1.194 \mathrm{E}+00$ & $1.017 \mathrm{E}+00$ & $1.678 \mathrm{E}+00$ \\
\hline Ionic Strength & $1.002 \mathrm{E}-01$ & $1.002 \mathrm{E}-01$ & $9.907 \mathrm{E}+00$ & $1.376 \mathrm{E}+00$ & $2.815 \mathrm{E}-01$ & $3.062 \mathrm{E}+00$ \\
\hline
\end{tabular}

